\title{
Tailoring the Mechanical Stiffness of DNA Nanostructures Using Engineered Defects
}

Supporting Information

Chanseok Lee ${ }^{* 1}$, Kyung Soo Kim ${ }^{* 1}$, Young-Joo Kim ${ }^{1}$, Jae Young Lee ${ }^{1}$, and ${ }^{*}$ Do-Nyun Kim ${ }^{1,2}$

${ }^{1}$ Department of Mechanical and Aerospace Engineering, Seoul National University, 1 Gwanak-ro, Gwanak-gu, Seoul 08826, Korea

${ }^{2}$ Institute of Advanced Machines and Design, Seoul National University,

1 Gwanak-ro, Gwanak-gu, Seoul 08826, Korea

†These authors contributed equally to this work.

*Corresponding author. Email: dnkim@snu.ac.kr 


\section{Contents}

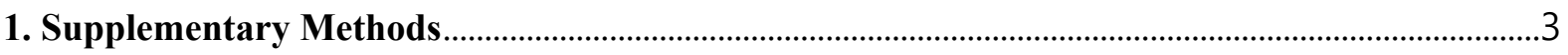

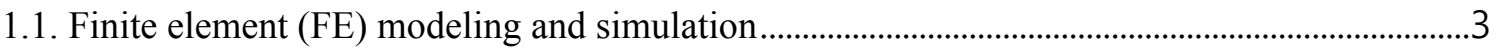

1.2. Molecular dynamics (MD) simulation ......................................................................................

2. Design and characterization of $4 \mathrm{HB}$ and $6 \mathrm{HB}$ structures ..............................................................

2-1. Layout of engineered defects...................................................................................................

2-2. Experimental characterization of 4HB designs ......................................................................9

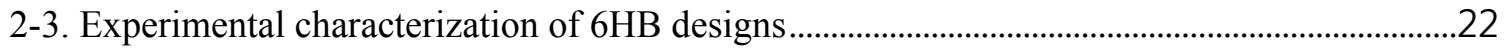

3. Bending persistence length measurement from the monomer contour images ……………….....43

4. Anisotropic gap distribution of $6 \mathrm{HB}$ design in longitudinal direction ............................................48

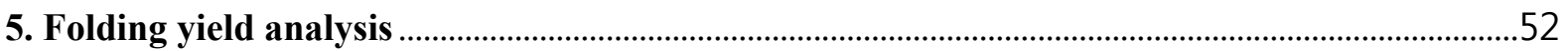

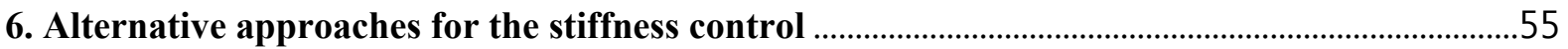

7. Finite element modeling of defect-engineered DNA nanostructures ................................................58

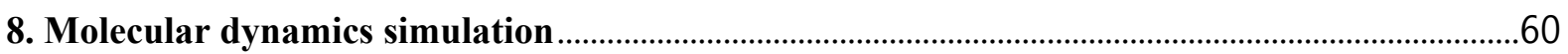

9. FE simulation on various cross-sections with engineered defects ....................................................68

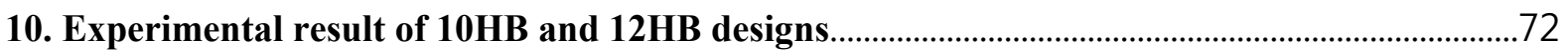

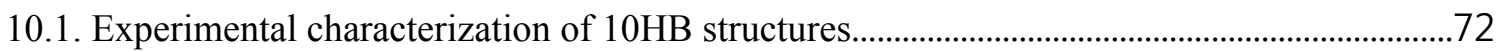

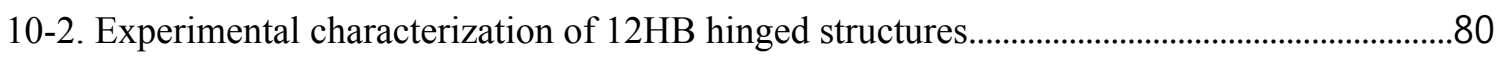

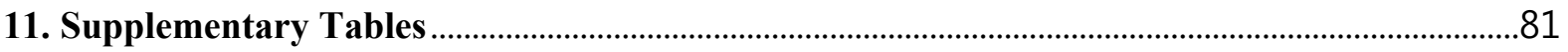

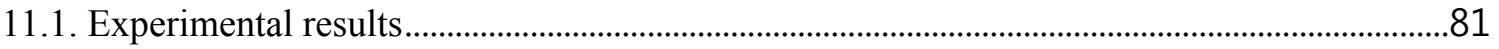

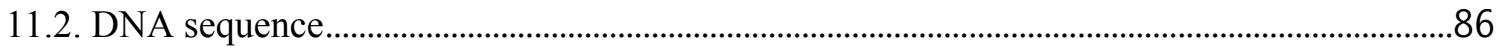

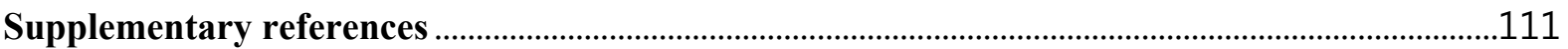




\section{Supplementary Methods}

\subsection{Finite element (FE) modeling and simulation}

FE model for DNA nanostructures. We constructed a FE model to predict bending persistence length $\left(\mathrm{L}_{\mathrm{p}}\right)$ of defect-engineered DNA nanostructures. From caDNAno ${ }^{1}$ design files, connectivity information of all bases was obtained. A base pair (BP), or a base was defined as a node, whose mass density is assumed to be on average $0.8 \mathrm{~g} / \mathrm{cm}^{3}$ and $0.4 \mathrm{~g} / \mathrm{cm}^{3}$, respectively. Two successive nodes along a helix were connected by a beam element, and it represents one of four different structural motifs: a normal DNA duplex, a nicked DNA duplex, a DNA duplex at Holliday junction (HJcore), a ssDNA gap. Here, all elements have the regular geometry of B-form DNA (diameter of $2.25 \mathrm{~nm}$ in honeycomb lattice ${ }^{2}$ and $2.5 \mathrm{~nm}$ in square lattice ${ }^{3}$ packing, axial rise of $0.34 \mathrm{~nm}$, and helicity of $10.5 \mathrm{BP} / \mathrm{turn}$, respectively). But in terms of mechanical properties, only the normal and nicked DNA duplexes have the regular values (stretch modulus of $1100 \mathrm{pN}$, bending rigidity of $230 \mathrm{pNnm}^{2}$, and torsional rigidity of $460 \mathrm{pNnm}^{2}$, respectively). ${ }^{4}$ The HJcore and ssDNA gap element have more flexible mechanical properties than those of the normal DNA duplexes by adopting a scale factor (SF) to those of the normal DNA duplex. For the ssDNA gap element, we additionally considered the dependency of mechanical properties on the length of the gap. A crossover connecting two adjacent helices was modelled by another beam element, whose SFs were determined in our previous work. ${ }^{5}$

Determination of scale factors for various structural motifs. For the HJcore element, we determined SFs by optimizing them to obtain similar $\mathrm{L}_{\mathrm{p}}$ values for both $4 \mathrm{HB}$ on square lattice and $6 \mathrm{HB}$ on honeycomb lattice with no gap to those measured from experiments. After the optimization of HJcore element, we determined SFs for ssDNA gap elements by optimizing them to obtain similar $\mathrm{L}_{\mathrm{p}}$ values for both gapped $4 \mathrm{HB}$ with square lattice and $6 \mathrm{HB}$ with honeycomb lattice to those measured from experiments. As the length-dependent softening behavior of ssDNA gaps was observed in experimental results, we computed different SFs for 1, 3 and 5-nt-long gaps, respectively. Then, SFs for 2 and 4-ntlong gaps were from a quadratic interpolation with SFs of 1,3 and 5-nt-long gaps.

To determine SFs for HJcore and ssDNA gap elements, we solved the following optimization problem using the 'fmincon' function in Matlab R2016b (MathWorks Inc.) with numerically calculated gradients

$$
\begin{aligned}
& \text { Minimize: } \mathrm{f}_{0}=\sqrt{\frac{1}{2} \sum_{\mathrm{i}=1}^{2}\left(\mathrm{~L}_{\mathrm{p}, \mathrm{i}}^{\mathrm{EXP}}-\mathrm{L}_{\mathrm{p}, \mathrm{i}}^{\mathrm{FE}}(\mathrm{s})\right)^{2}}+\sqrt{\sum_{\mathrm{i}=1}^{\mathrm{N}}\left(\mathrm{A}_{\mathrm{i}}-\mathrm{mu}\right)^{2}} \\
& \text { (where } \mathrm{i}=1 \text { and } 2 \text { represent } 4 \mathrm{HB} \text { and } 6 \mathrm{HB}, \text { respectively) }
\end{aligned}
$$

Subject to: $10^{-6} \leq \mathrm{s}_{\mathrm{j}} \leq 10^{0}$

$$
\stackrel{\mathrm{I}}{\mathrm{S}}=\left[\begin{array}{lll}
\mathrm{SF}_{\mathrm{S}} & \mathrm{SF}_{\mathrm{B}} & \mathrm{SF}_{\mathrm{C}}
\end{array}\right]
$$

where $s_{j}$ is the variable, $L_{p, i}^{\text {EXP }}$ is the experimentally measured $L_{p}, L_{p, i}^{F E}$ is the $L_{p}$ from the FE model, $\mathrm{A}_{\mathrm{i}}=\left|\mathrm{L}_{\mathrm{p}, \mathrm{i}}^{\mathrm{EXP}}-\mathrm{L}_{\mathrm{p}, \mathrm{i}}^{\mathrm{FE}}\right|$, and $\mathrm{mu}=\frac{1}{2} \sum_{\mathrm{i}=1}^{2} \mathrm{~A}_{\mathrm{i}}$, respectively.

Detailed SF values of each elements obtained from optimization procedure, were summarized in Supplementary Table 3. 
Calculation of bending persistence length for DNA nanostructures. Normal mode analysis (NMA) was performed at the straight configuration to compute the lowest 20 normal modes of DNA nanostructures. Intrinsic global twist of square-lattice packed structures ${ }^{3}$ was not considered. Given the FE model for a DNA nanostructure under free boundary condition, a generalized eigenvalue problem,

$$
\mathrm{Ku}=\lambda \mathrm{Mu}
$$

where $K$ is the stiffness matrix, $M$ is the mass matrix and the eigenvalue $\lambda=\omega^{2}$ was defined. The Subspace iteration procedure ${ }^{6-7}$ was used to solve the eigenvalue problem using $2 \mathrm{~N}_{\mathrm{m}}$ iteration vectors, where $\mathrm{N}_{\mathrm{m}}$ denotes the number of eigenmodes to be calculated. Among the eigenvalues obtained, only the two eigenvalues for the first bending modes were selected to calculate bending stiffness (EI) of DNA nanostructures.

From the Euler-Lagrange equation for a beam representing effectively DNA nanostructures, we could obtain the following free vibration equation. ${ }^{8}$

$$
\mathrm{EI} \frac{\mathrm{d}^{4} \mathrm{w}}{\mathrm{dx} \mathrm{x}^{4}}+\mu \omega^{2} \mathrm{w}=0
$$

where $\mathrm{w}$ describes the lateral deflection of the beam, $\mathrm{x}$ represents the axial position, and $\mu$ is the mass per unit length of the beam, respectively. Solving numerically the above equation, we can obtain the natural frequencies of the first bending vibration $\left(\omega_{\mathrm{B}}\right)$

$$
\omega_{\mathrm{B}}=\frac{4.733^{2}}{\mathrm{~L}^{2}} \sqrt{\frac{\mathrm{EI}}{\mu}}
$$

where $\mathrm{L}$ is the length of the beam. Then, EI of the beam becomes

$$
\mathrm{EI}=\mu \omega_{\text {в }}^{2} \frac{\mathrm{L}^{4}}{4.733^{4}}=\frac{\mathrm{m}}{\mathrm{L}} \lambda^{2} \frac{\mathrm{L}^{4}}{4.733^{4}}=\mathrm{m} \lambda_{\text {в }}^{2} \frac{\mathrm{L}^{3}}{4.733^{4}}
$$

where $m$ is the total mass of DNA nanostructures and $\lambda_{B}$ is the eigenvalues for the first bending mode. Since the $\mathrm{L}_{\mathrm{p}}$ is defined as

$$
\mathrm{L}_{\mathrm{p}}=\frac{\mathrm{EI}}{\mathrm{k}_{\mathrm{b}} \mathrm{T}}
$$

where $\mathrm{k}_{\mathrm{b}}$ is the Boltzmann constant and $\mathrm{T}$ is absolute temperature, assumed to be $298 \mathrm{~K}$. Then, the $\mathrm{L}_{\mathrm{p}}$ of DNA nanostructures becomes

$$
\mathrm{L}_{\mathrm{p}}=\mathrm{m} \lambda_{\mathrm{B}}^{2} \frac{\mathrm{L}^{3}}{4.733^{4} \mathrm{k}_{\mathrm{B}} \mathrm{T}}
$$

In three-dimensional structure, there are always two first bending modes. Therefore, we defined the first bending mode with a smaller eigenvalue as a major bending mode $\left(\mathrm{L}_{\mathrm{p}, 1}\right)$ and the other with a larger eigenvalue as a minor bending mode $\left(\mathrm{L}_{\mathrm{p}, 2}\right)$. Using both bending modes, we defined an effective bending persistence length $\left(L_{p, e}\right)$, which we compared with experimentally measured $L_{p}\left(L_{p, i}^{E X P}\right)$.

$$
\mathrm{L}_{\mathrm{p}, \mathrm{e}}=\frac{2}{1 / \mathrm{L}_{\mathrm{p}, 1}+1 / \mathrm{L}_{\mathrm{p}, 2}}
$$




\subsection{Molecular dynamics (MD) simulation}

General protocol. The starting atomic structures of non-gapped and gapped 6HB designs were generated using caDNAno ${ }^{1}$ and CanDo. ${ }^{4}$ Each atomic structure was explicitly solvated using the TIP3P water model $^{9}$ with a distance more than $15 \AA$ from the structure and boundary. It results in approximately $100 \AA \times 100 \AA \times 320 \AA$ of cubic water box and neutralized to an ion concentration of 20 $\mathrm{mM} \mathrm{MgCl} 2 .{ }^{10} \mathrm{MD}$ simulation was then performed using NAMD ${ }^{11}$ with the CHARMM36 force field, ${ }^{12}$ periodic boundary conditions, the integration time step of $2 \mathrm{fs}$, and short-range electrostatic potentials with $12 \AA$ cut-off. The long-range electrostatic interactions were computed using the Particle-MeshEwald (PME) scheme ${ }^{13}$ with the grid size of $1 \AA$. The potential energy of each system was minimized using the conjugate gradient method. Each structure was simulated for $320 \mathrm{~ns}$ under the isobaricisothermal (NPT) ensemble, and trajectories of the final $200 \mathrm{~ns}$ were used for further analysis.

\section{Cross-section analysis of $6 \mathrm{HB}$ designs}

(A)

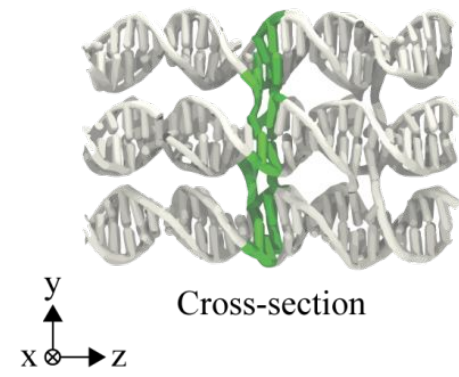

(B)

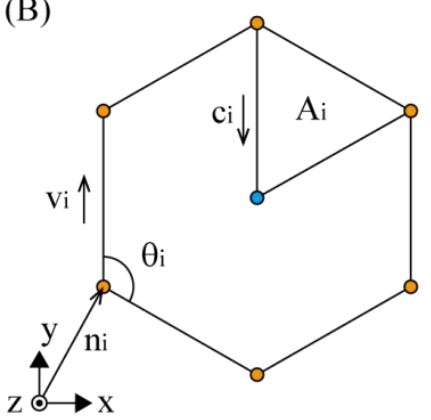

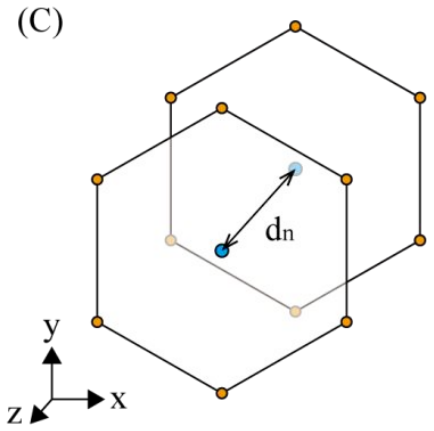

In order to obtain the fluctuation of cross-sectional shape 6-helix-bundle structures, we first selected five cross-sections with equidistant intervals (the green bases in A). For the two base pairs of a crosssection, their origins were calculated following the 3DNA definition ${ }^{14}$ to provide a center point by averaging them. The three-dimensional dynamics of six center points was reduced to two-dimensional planar motion, by introducing in a projected hexagonal plane with six vertices (orange points in B), which minimizes the distance from the plane to six center points (the hexagonal plane in B). Vertex vectors $\left(\mathrm{n}_{\mathrm{i}}\right)$ were then obtained representing the position of each vertex, and edge vectors $\left(\mathrm{v}_{\mathrm{i}}\right)$ were determined using connected two vertices as

$$
\mathrm{v}_{\mathrm{i}}=\mathrm{n}_{\mathrm{i}+1}-\mathrm{n}_{\mathrm{i}}
$$

The interior angle in hexagon $\left(\theta_{\mathrm{i}}\right)$ was calculated using two successive vertex vectors as

$$
\theta_{i}=\cos ^{-1}\left[\left(v_{i} \cdot v_{i+1}\right) /\left(\left|v_{i}\right|\left|v_{i+1}\right|\right)\right]
$$

suggesting that the average of the angles should be $120^{\circ}$ and their standard deviation implies the angular fluctuation of a cross-section. A sectional area in a hexagon $\left(\mathrm{A}_{\mathrm{i}}\right)$ was derived as

$$
A_{i}=\left|c_{i} \times v_{i}\right| / 2
$$

where the hexagonal center vector $\left(c_{i}\right)$ was defined as a vector from the vertex to the hexagonal center point, resulting in the area of the hexagonal plane as the summation of six sectional areas. The distance between hexagonal planes $\left(d_{n}\right)$ was defined as the distance between hexagonal center points $(C)$. This cross-section analysis described above was performed for all snapshots of final 200-ns-long MD trajectories, therefore providing the probability density functions or standard deviations of cross- 
sectional variables presented in Fig. $3 \mathrm{e}$ and $3 \mathrm{f}$ in the manuscript.

Principal component analysis. The principal component analysis (PCA) was performed using the final 200-ns-long MD trajectories in equilibrium. ${ }^{15}$ When the coordinates of phosphorus atoms in a 6-helixbundle structure were denoted as $\mathrm{x}(\mathrm{t})$, the covariance matrix $(\sigma)$ is determined as ${ }^{16}$

$$
\sigma=\langle(\mathrm{x}(\mathrm{t})-|\mathrm{x}(\mathrm{t})|) \otimes(\mathrm{x}(\mathrm{t})-|\mathrm{x}(\mathrm{t})|)\rangle
$$

where $\otimes$ indicates the tensor product and the angle bracket represents the average of the vector. The square-root-mass-weight matrix $(\Sigma)$ is now obtained as

$$
\Sigma=\sqrt{\mathrm{M}}_{\sigma} \sqrt{\mathrm{M}}
$$

where $\mathrm{M}$ is a diagonal matrix with the atomic weight of phosphorus atoms. Then the eigenvalues $\left(\lambda_{\mathrm{n}}\right)$ of the $\mathrm{n}^{\text {th }}$ mode were obtained by diagonalizing the square-root-mass-weight matrix, providing the quasi-harmonic eigenfrequencies $\left(\omega_{n}\right)$ as

$$
\omega_{\mathrm{n}}=\sqrt{\mathrm{k}_{\mathrm{B}} \mathrm{T} / \lambda_{\mathrm{n}}}
$$

where $\mathrm{k}_{\mathrm{B}}$ and $\mathrm{T}$ indicate the Boltzmann constant and absolute temperature, respectively. The dynamic Euler-Bernoulli beam model with the boundary conditions of free-free ends suggests that the elastic bending stiffness $\left(\mathrm{EI}_{n}\right)$ are approximately calculated using the eigenfrequencies for the $\mathrm{n}^{\text {th }}$ mode as

$$
\mathrm{EI}_{\mathrm{n}}=\left(\mathrm{ML}^{3} \omega_{\mathrm{n}}^{2}\right) /\left(\beta_{\mathrm{n}} \mathrm{L}\right)^{4}
$$

where $M$ and $L$ are the mass and axial length of $6 \mathrm{HB}$ structures, respectively, and $\beta_{\mathrm{n}} \mathrm{L}$ is a predefined constant as 4.733 .

Base pairing ratio analysis. Base pairing analysis of the MD trajectories in $6 \mathrm{HB}$ structures was performed using the hydrogen-bond tool in VMD. ${ }^{17}$ Base pairing was determined whether a hydrogenbond was formed or not between the N1 atom of a purine base (Adenine or Guanine) and the N3 atom of a pyrimidine base (Thymine or Cytosine) with cut-off values of distance and angle as $4.0 \AA$ and $40^{\circ} .{ }^{18}$ Each MD snapshot of a base pair then provides zero (broken) or one (paired) value, providing the time-averaged hydrogen-bond ratio for final 200-ns-long MD trajectory as shown in Supplementary Fig. 61. 


\section{Design and characterization of $4 \mathrm{HB}$ and $6 \mathrm{HB}$ structures}

2-1. Layout of engineered defects

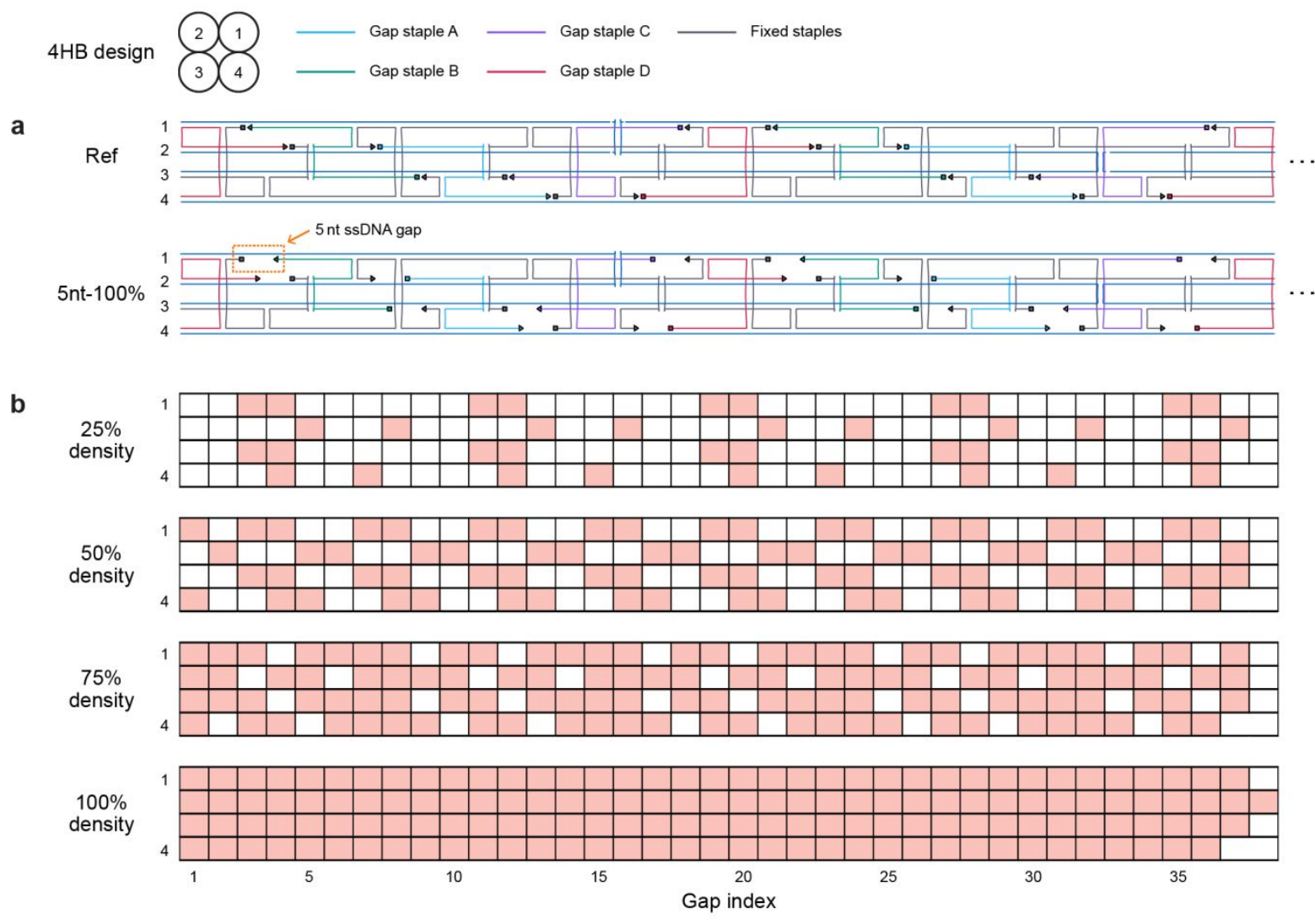

Supplementary Fig. 1. 4HB gap design. (a) Repetitive scaffold and staple route constituting 4HB with square-lattice packing. Rectangles and triangles indicate the 5' and 3' end of staple DNAs, respectively. Staples were categorized by the location of their nicks, and each group was represented by a different color. Refer to Supplementary Table 5 for all staple sequences. (b) Schematics that colored boxes show the positions where nicks located at the corresponding indices were changed to ssDNA gaps with programmed length. 

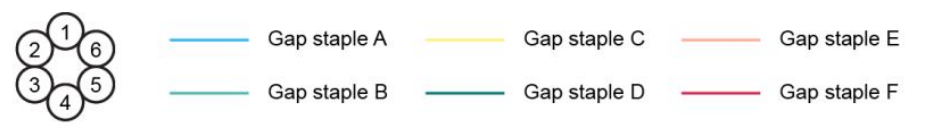

a

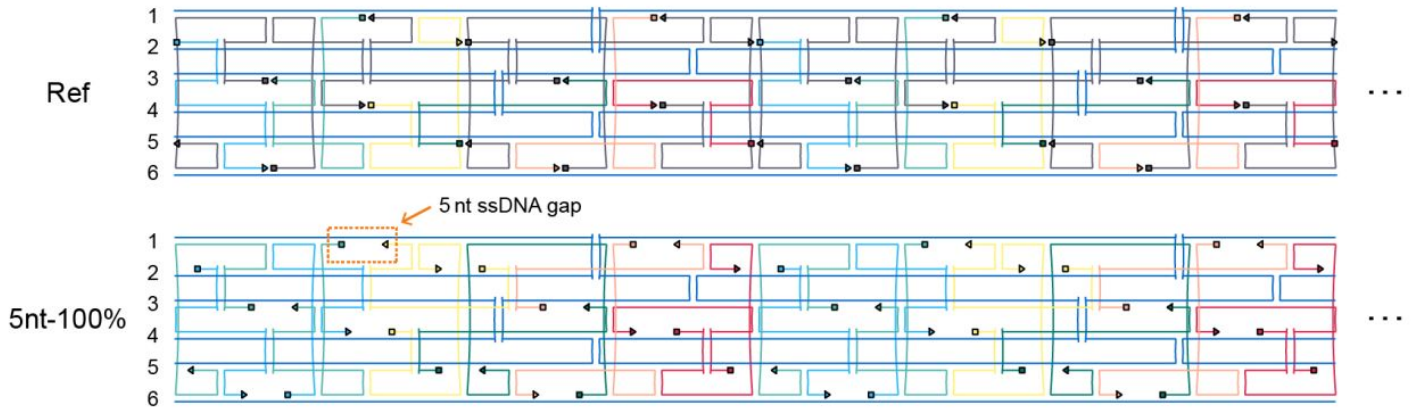

b

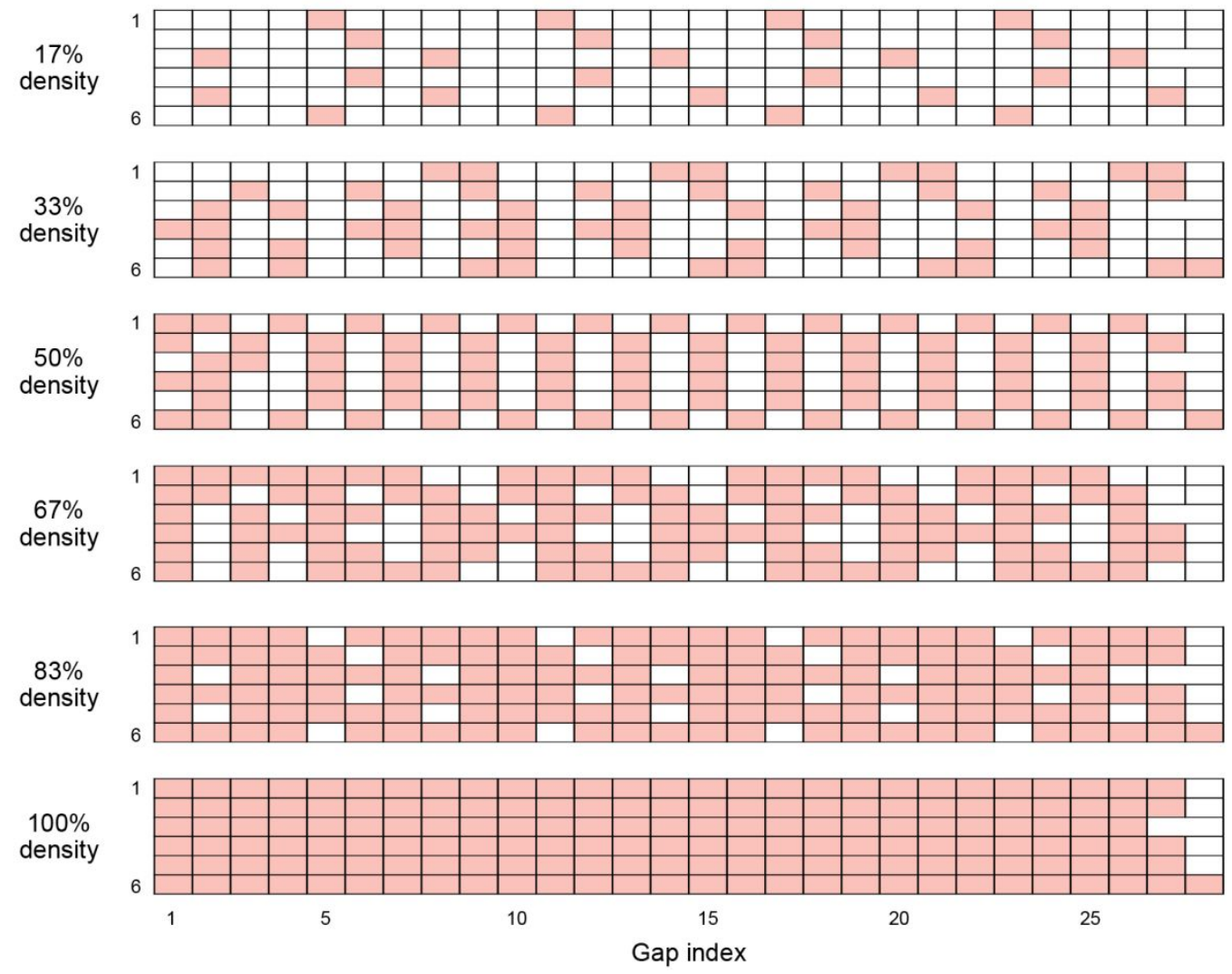

Supplementary Fig. 2. 6HB gap design. (a) Repetitive scaffold and staple route constituting 6HB with honeycomb-lattice packing. Rectangles and triangles indicate the 5' and 3' end of staple DNAs, respectively. Staples were categorized by the location of their nicks, and each group was represented by a different color. Refer to Supplementary Table 6 for all staple sequences. (b) Schematics that colored boxes show the positions where nicks located at the corresponding indices were changed to ssDNA gaps with programmed length. Seven nicks located at the both ends of the bundle were omitted in the diagram since they were not changed to ssDNA gaps throughout the gap density variations. 
2-2. Experimental characterization of $4 \mathrm{HB}$ designs

a
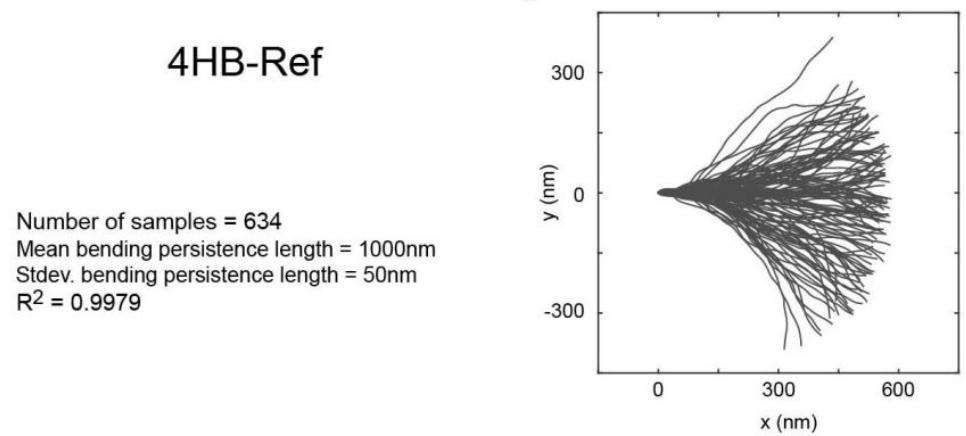

b

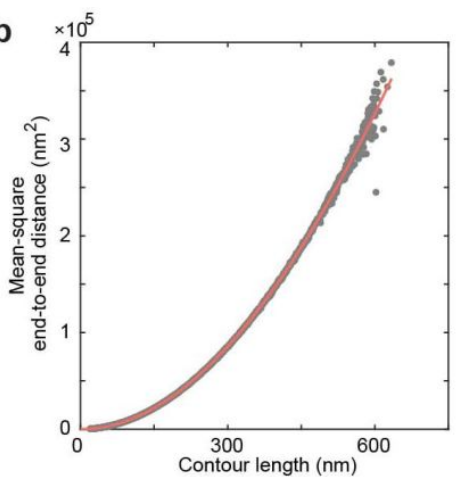

c

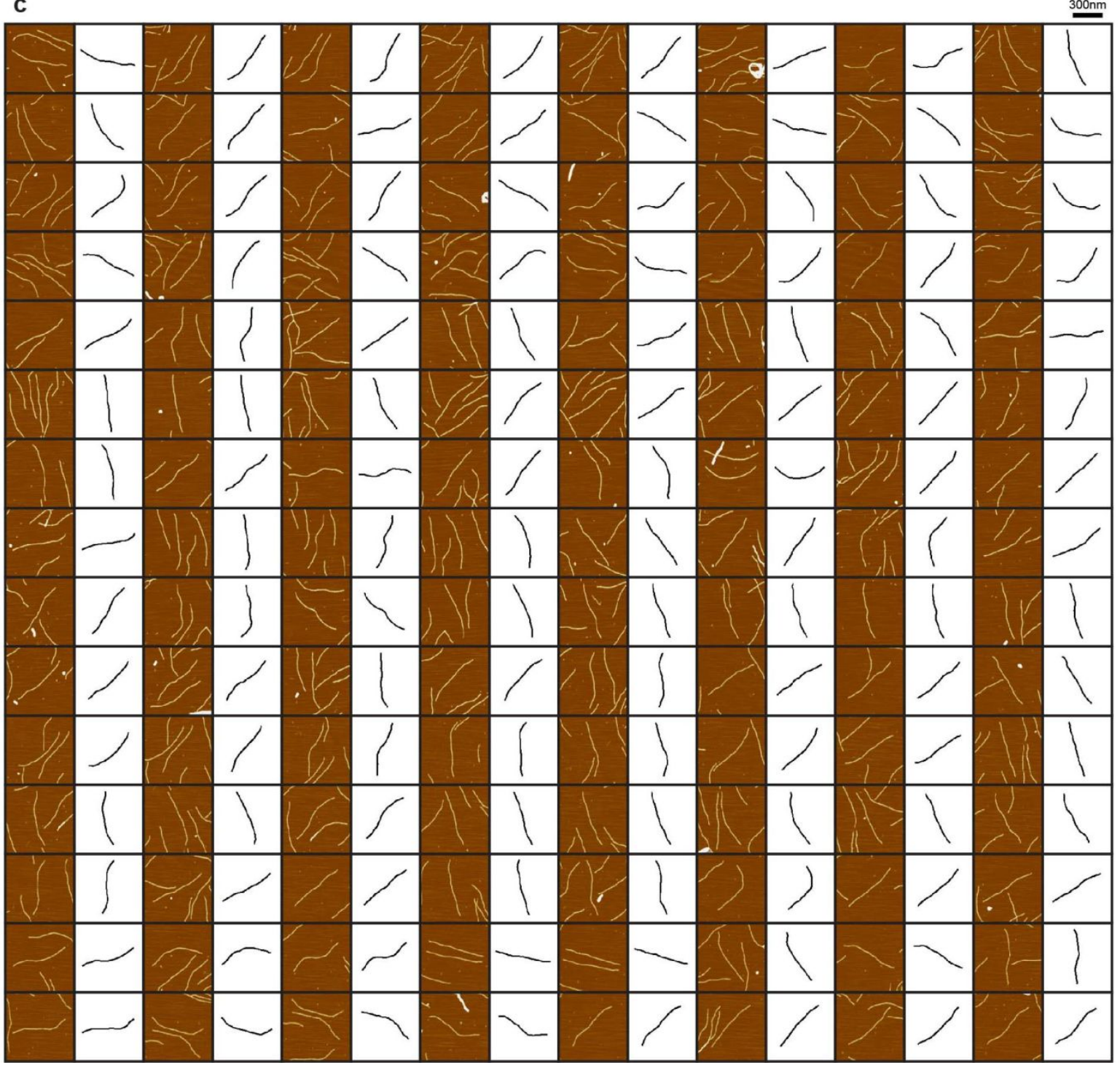

Supplementary Fig. 3. Experimental characterization of 4HB-Ref design. (a) Aligned contour distribution of 120 representative monomers. (b) Calculation of the mean bending persistence length by fitting all measured data. (c) AFM images and extracted monomer contours shown in (a). 
a

4HB-1nt-25\%

Number of samples $=396$

Mean bending persistence length $=900 \mathrm{~nm}$

Stdev. bending persistence length $=40 \mathrm{~nm}$

$R^{2}=0.9982$
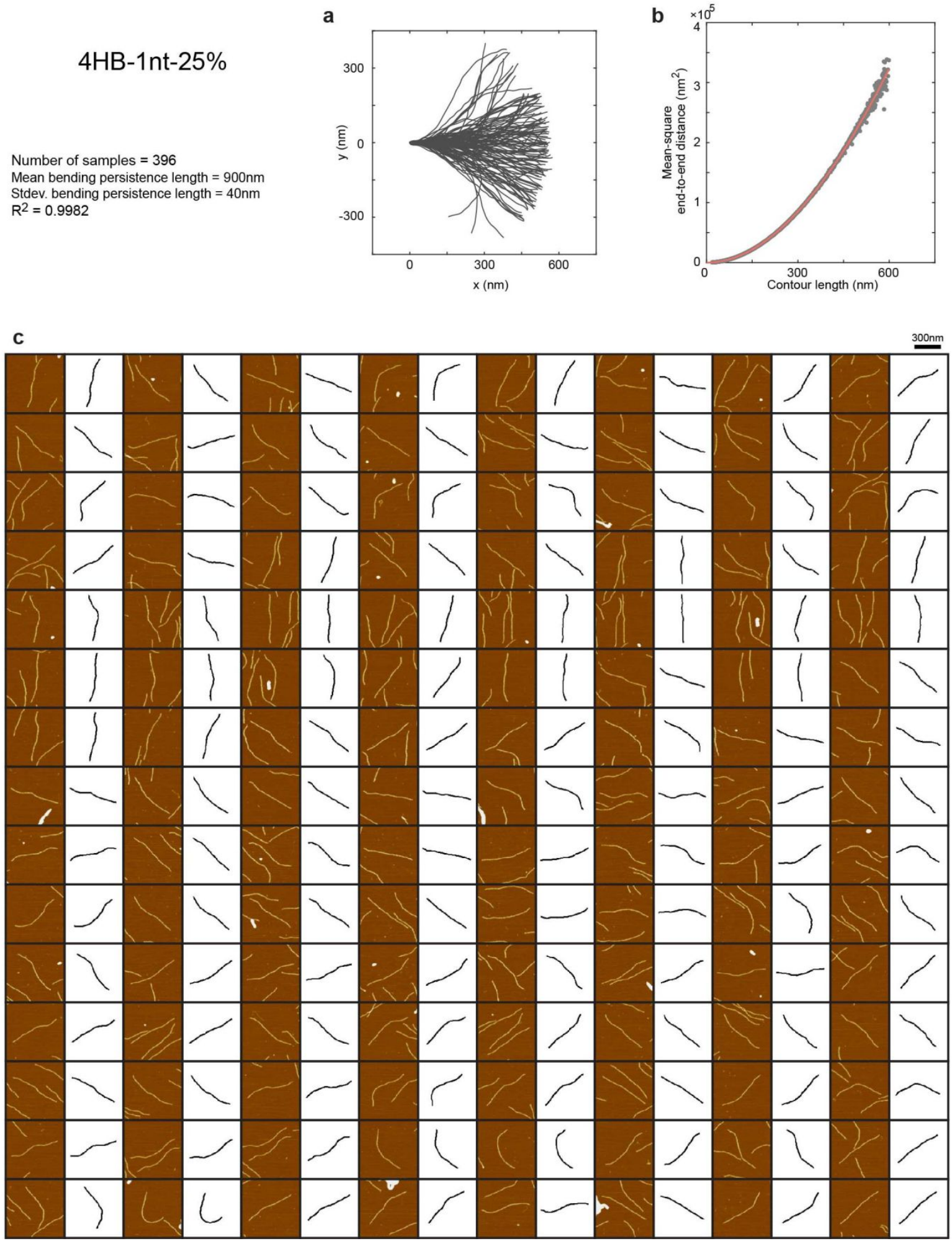

Supplementary Fig. 4. Experimental characterization of 4HB-1nt-25\% design. (a) Aligned contour distribution of 120 representative monomers. (b) Calculation of the mean bending persistence length by fitting all measured data. (c) AFM images and extracted monomer contours shown in (a). 
a

4HB-1nt-50\%

Number of samples $=453$

Mean bending persistence length $=840 \mathrm{~nm}$

Stdev. bending persistence length $=40 \mathrm{~nm}$

$R^{2}=0.9979$
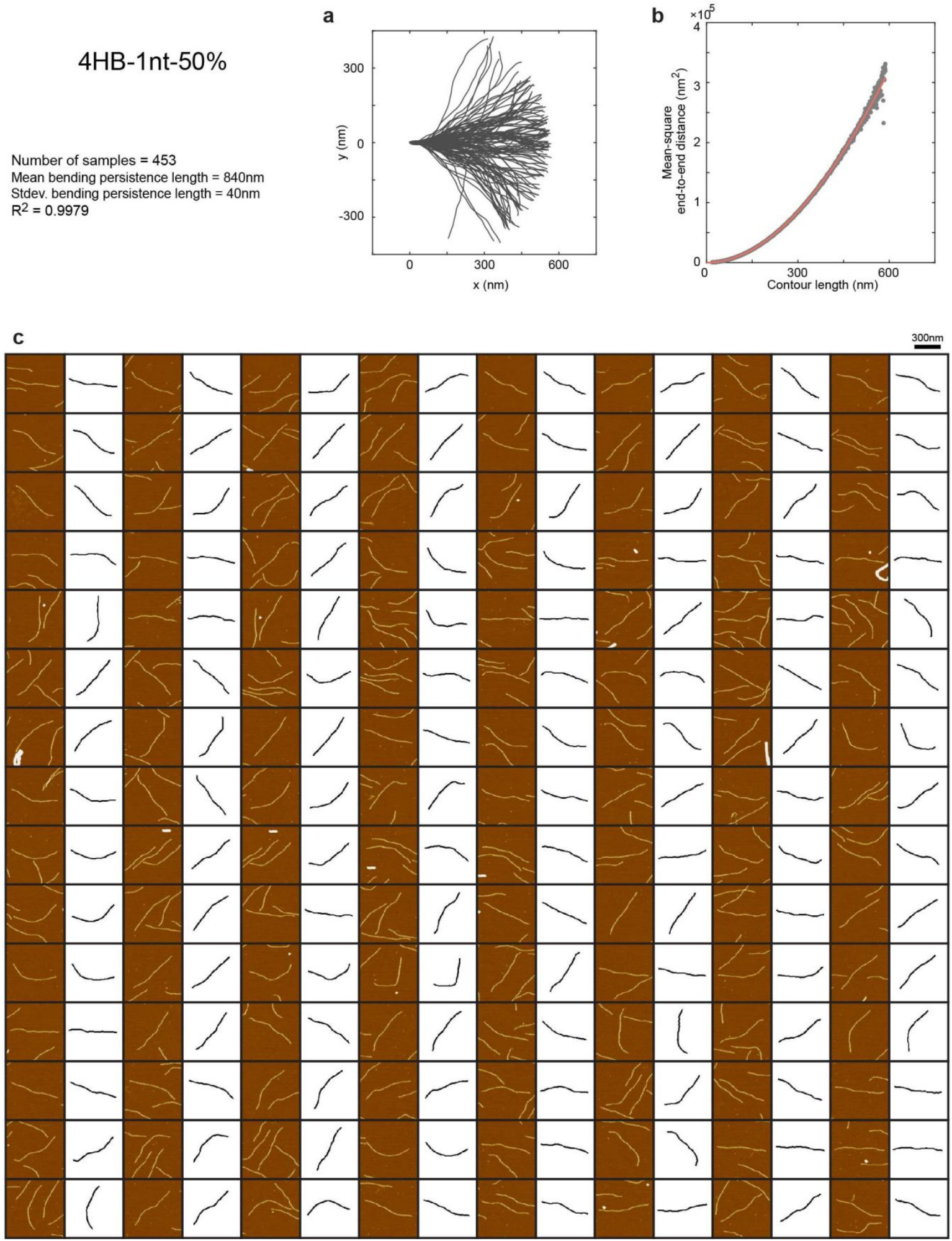

Supplementary Fig. 5. Experimental characterization of 4HB-1nt-50\% design. (a) Aligned contour distribution of 120 representative monomers. (b) Calculation of the mean bending persistence length by fitting all measured data. (c) AFM images and extracted monomer contours shown in (a). 
a

4HB-1nt-75\%

Number of samples $=619$

Mean bending persistence length $=800 \mathrm{~nm}$

Stdev. bending persistence length $=40 \mathrm{~nm}$

$R^{2}=0.9979$
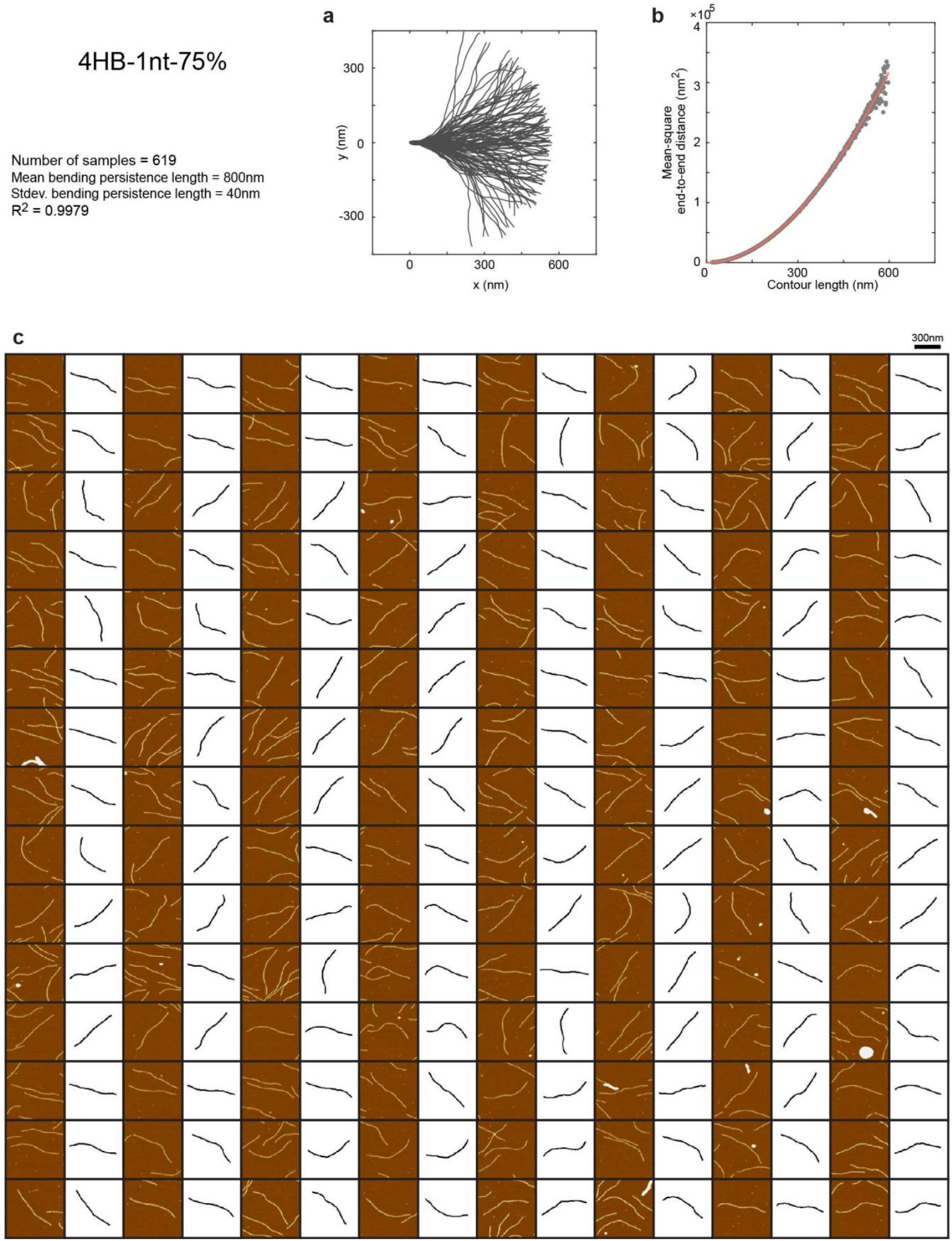

Supplementary Fig. 6. Experimental characterization of 4HB-1nt-75\% design. (a) Aligned contour distribution of 120 representative monomers. (b) Calculation of the mean bending persistence length by fitting all measured data. (c) AFM images and extracted monomer contours shown in (a). 
a

4HB-1nt-100\%

Number of samples $=399$

Mean bending persistence length $=760 \mathrm{~nm}$

Stdev. bending persistence length $=40 \mathrm{~nm}$

$R^{2}=0.9911$
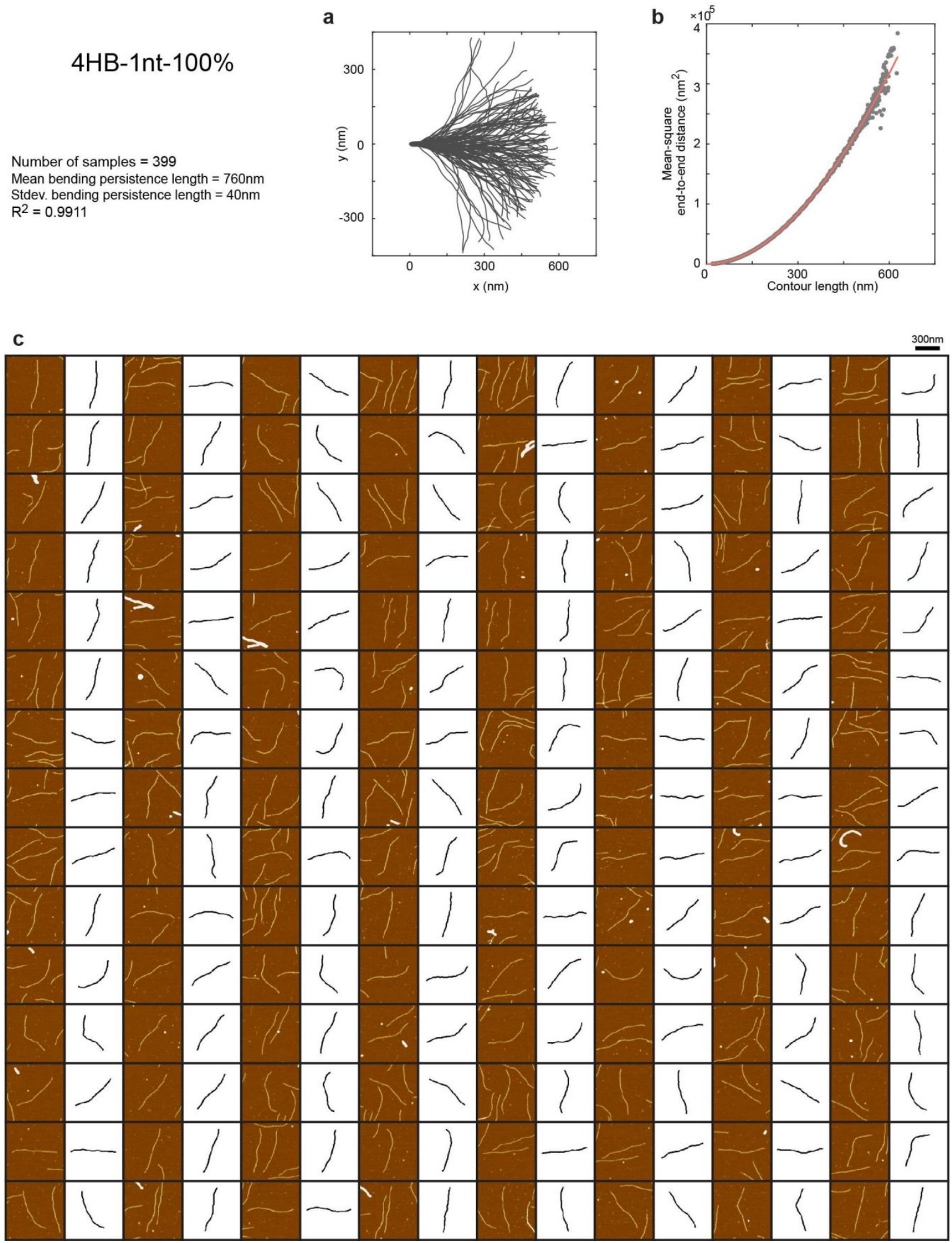

Supplementary Fig. 7. Experimental characterization of $4 \mathrm{HB}-1 \mathrm{nt}-100 \%$ design. (a) Aligned contour distribution of 120 representative monomers. (b) Calculation of the mean bending persistence length by fitting all measured data. (c) AFM images and extracted monomer contours shown in (a). 
a

4HB-3nt-25\%

Number of samples $=506$

Mean bending persistence length $=740 \mathrm{~nm}$

Stdev. bending persistence length $=40 \mathrm{~nm}$

$R^{2}=0.9972$
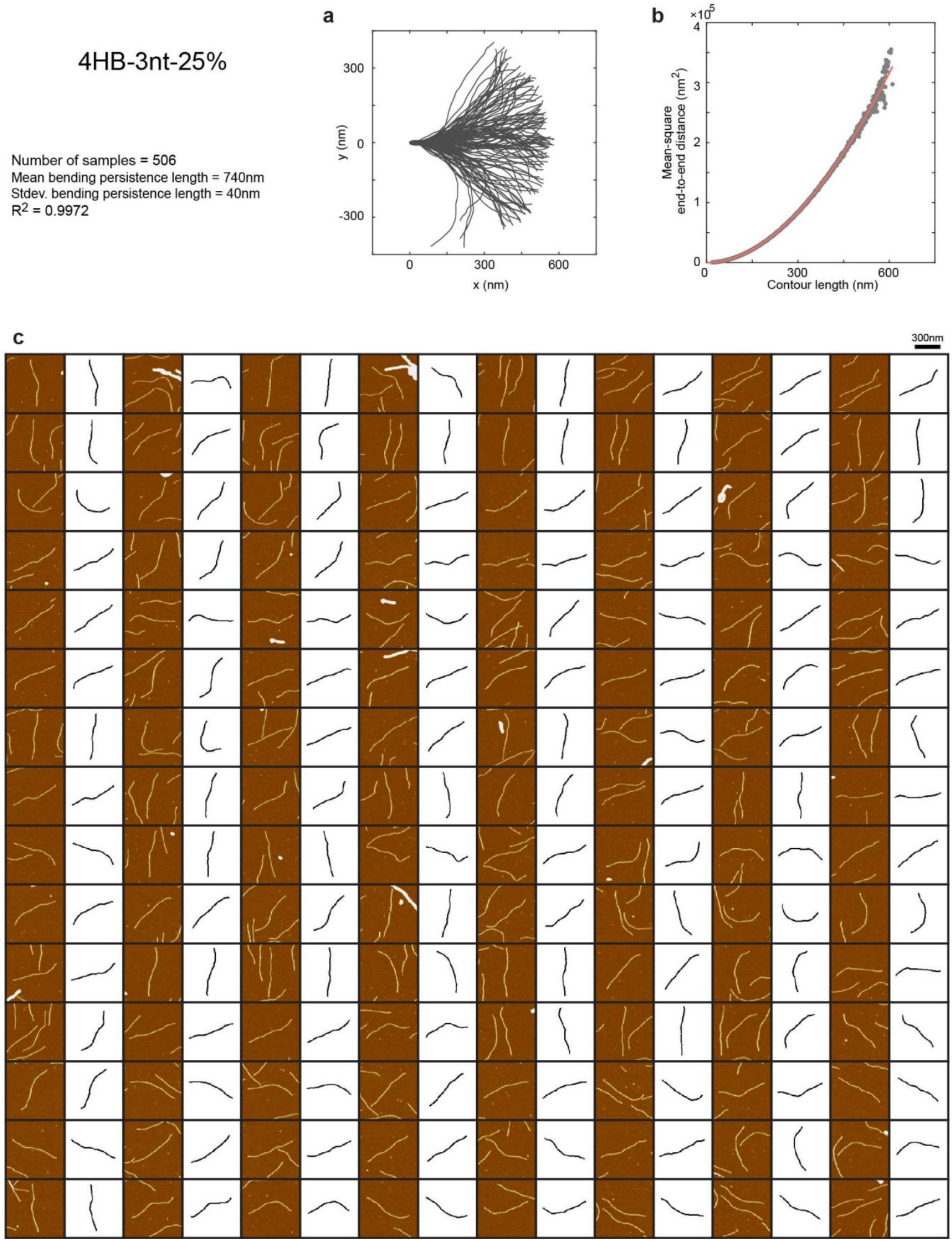

Supplementary Fig. 8. Experimental characterization of 4HB-3nt-25\% design. (a) Aligned contour distribution of 120 representative monomers. (b) Calculation of the mean bending persistence length by fitting all measured data. (c) AFM images and extracted monomer contours shown in (a). 
a

4HB-3nt-50\%

Number of samples $=563$

Mean bending persistence length $=650 \mathrm{~nm}$

Stdev. bending persistence length $=30 \mathrm{~nm}$

$R^{2}=0.9938$
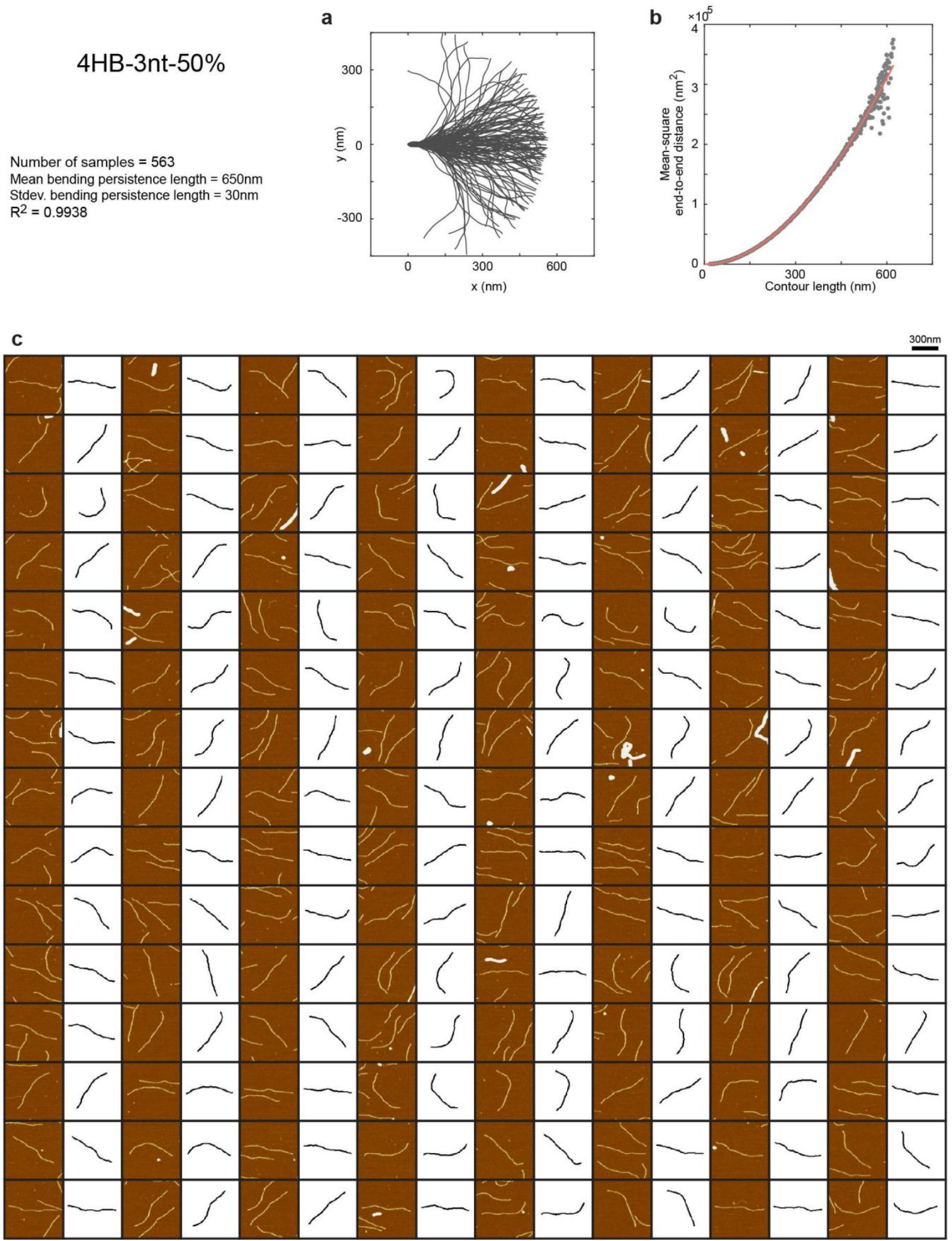

Supplementary Fig. 9. Experimental characterization of 4HB-3nt-50\% design. (a) Aligned contour distribution of 120 representative monomers. (b) Calculation of the mean bending persistence length by fitting all measured data. (c) AFM images and extracted monomer contours shown in (a). 
a

4HB-3nt-75\%

Number of samples $=\mathbf{5 8 9}$

Mean bending persistence length $=470 \mathrm{~nm}$

Stdev. bending persistence length $=20 \mathrm{~nm}$

$R^{2}=0.9967$
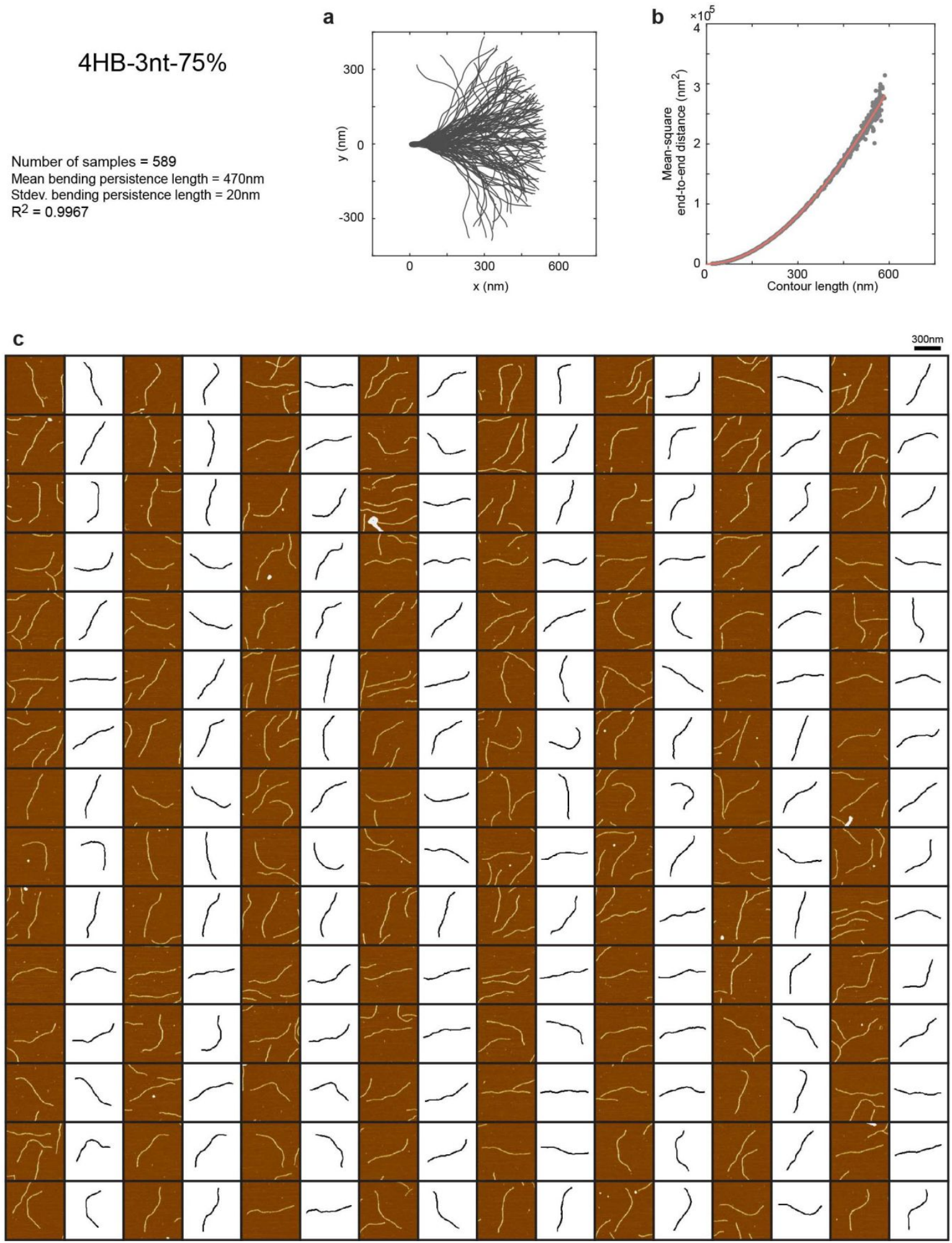

Supplementary Fig. 10. Experimental characterization of 4HB-3nt-75\% design. (a) Aligned contour distribution of 120 representative monomers. (b) Calculation of the mean bending persistence length by fitting all measured data. (c) AFM images and extracted monomer contours shown in (a). 
4HB-3nt-100\%

Number of samples $=365$

Mean bending persistence length $=400 \mathrm{~nm}$

Stdev. bending persistence length $=20 \mathrm{~nm}$

$R^{2}=0.9984$ a

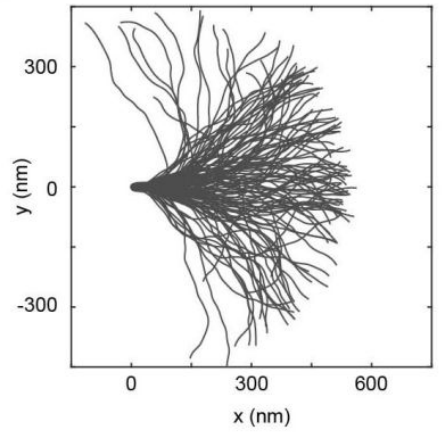

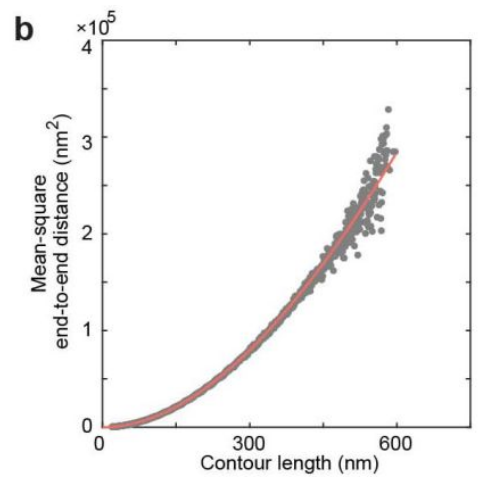

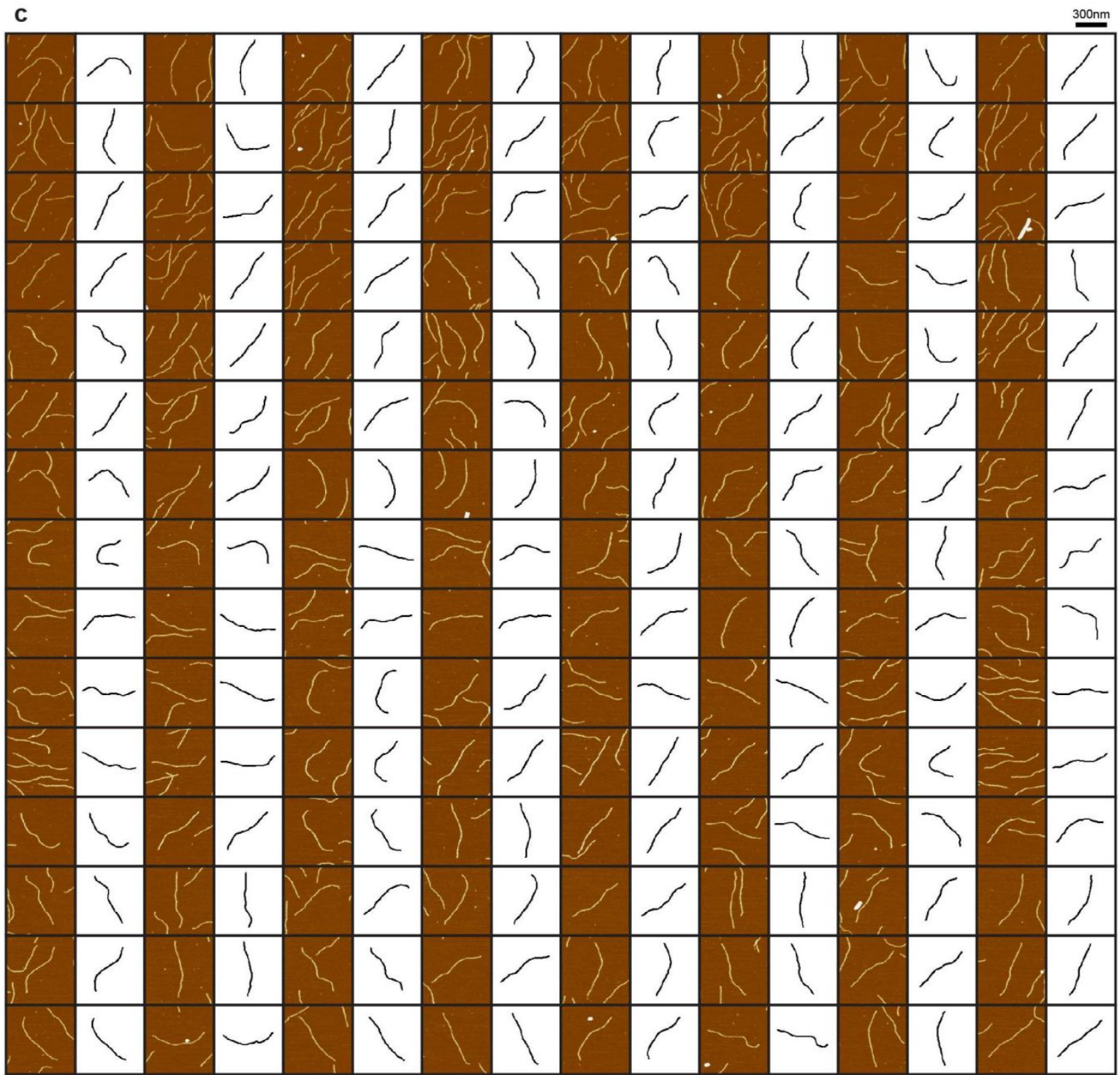

Supplementary Fig. 11. Experimental characterization of 4HB-3nt-100\% design. (a) Aligned contour distribution of 120 representative monomers. (b) Calculation of the mean bending persistence length by fitting all measured data. (c) AFM images and extracted monomer contours shown in (a). 
a

4HB-5nt-25\%

Number of samples $=231$

Mean bending persistence length $=660 \mathrm{~nm}$

Stdev. bending persistence length $=30 \mathrm{~nm}$

$R^{2}=0.9923$
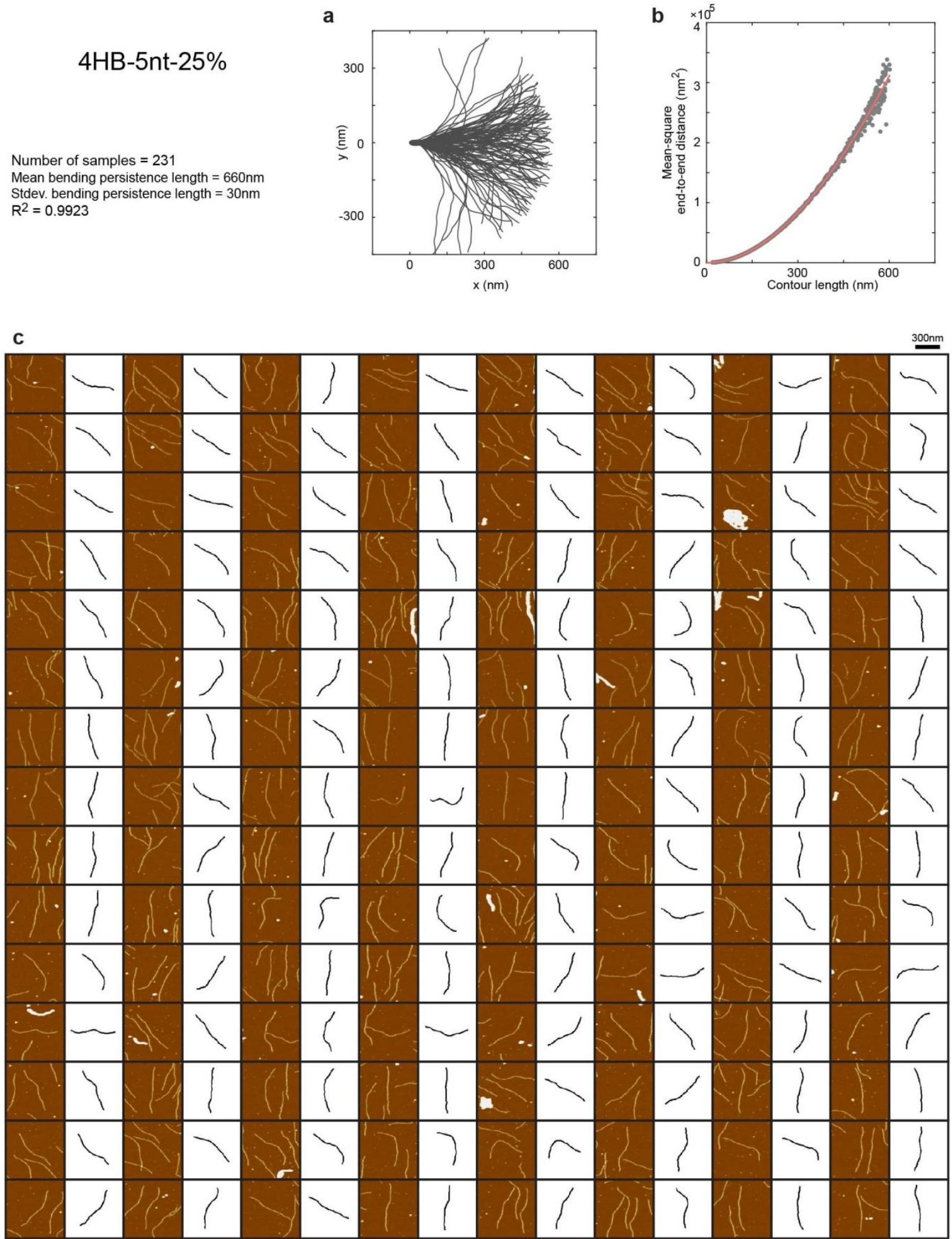

Supplementary Fig. 12. Experimental characterization of 4HB-5nt-25\% design. (a) Aligned contour distribution of 120 representative monomers. (b) Calculation of the mean bending persistence length by fitting all measured data. (c) AFM images and extracted monomer contours shown in (a). 
a

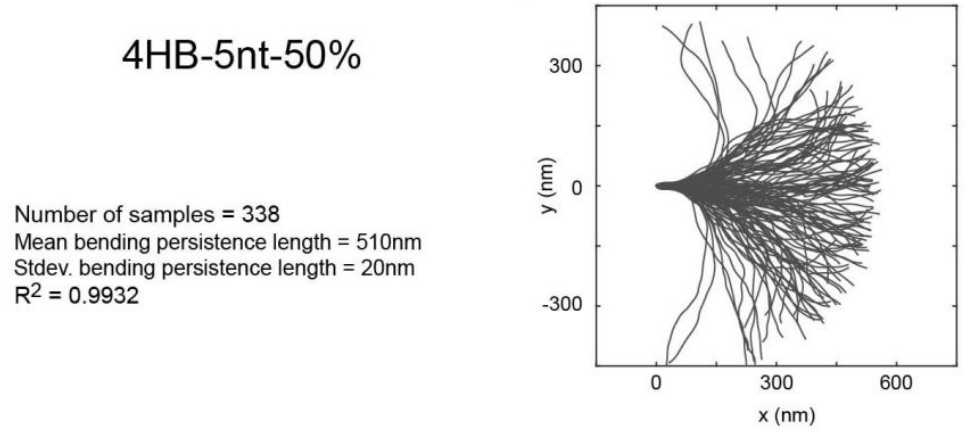

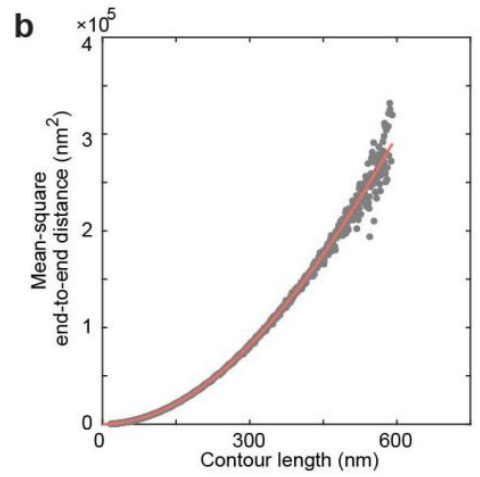

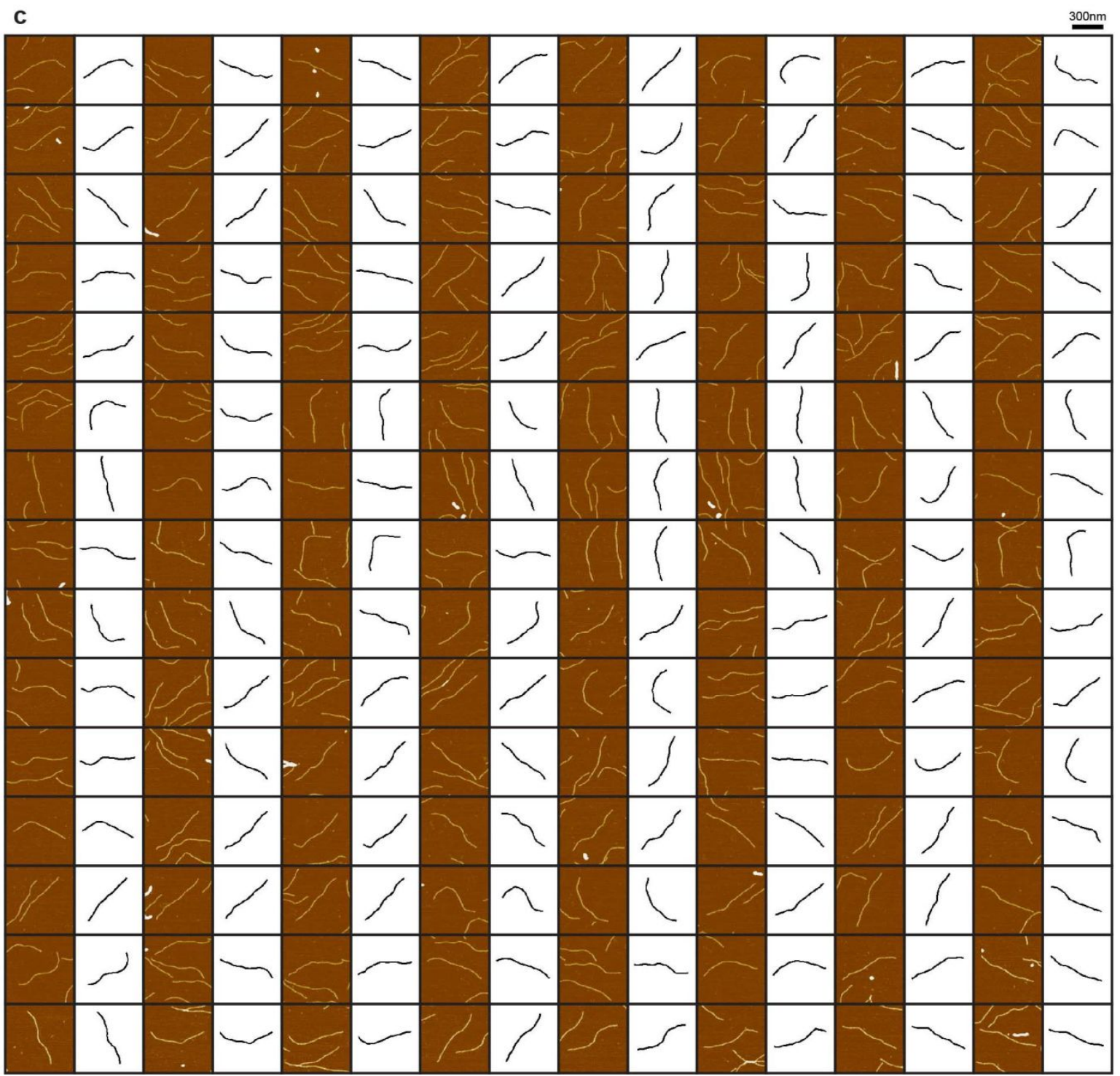

Supplementary Fig. 13. Experimental characterization of $4 \mathrm{HB}-5 \mathrm{nt}-50 \%$ design. (a) Aligned contour distribution of 120 representative monomers. (b) Calculation of the mean bending persistence length by fitting all measured data. (c) AFM images and extracted monomer contours shown in (a). 
a

4HB-5nt-75\%

Number of samples $=\mathbf{3 7 5}$

Mean bending persistence length $=340 \mathrm{~nm}$

Stdev. bending persistence length $=10 \mathrm{~nm}$

$R^{2}=0.9929$

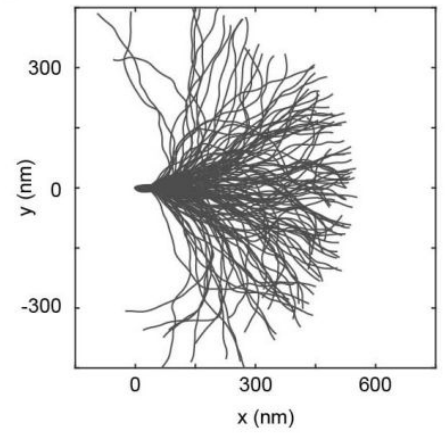

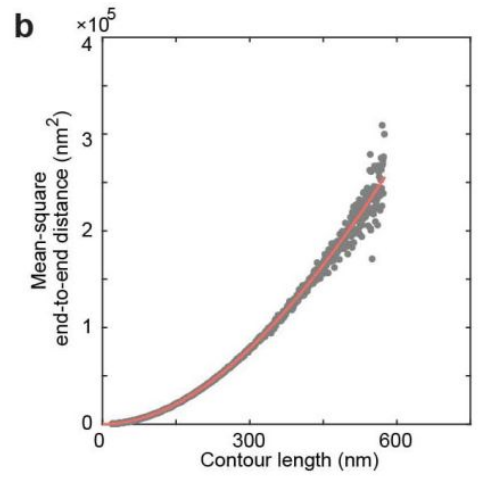

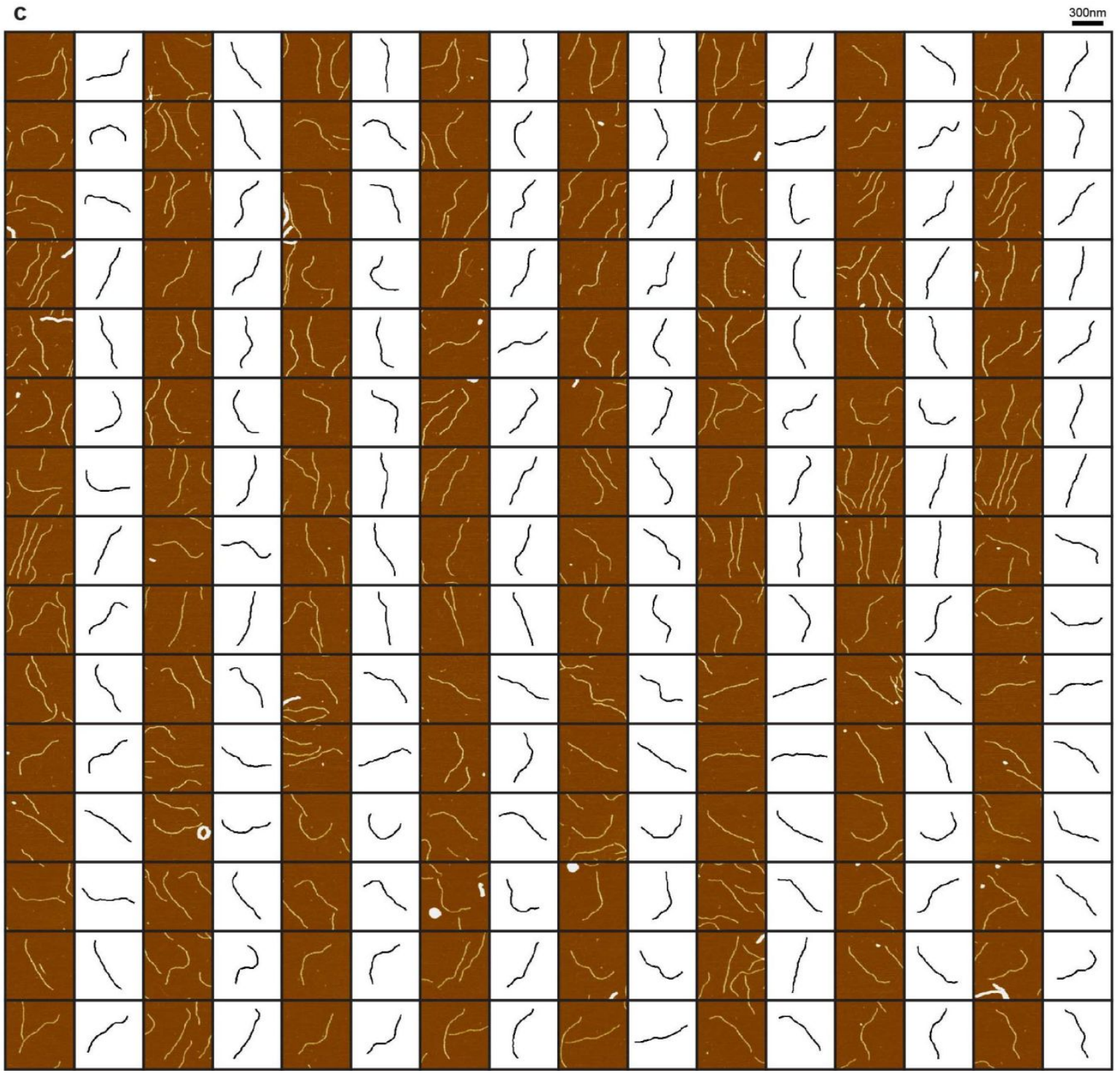

Supplementary Fig. 14. Experimental characterization of $4 \mathrm{HB}-5 \mathrm{nt}-75 \%$ design. (a) Aligned contour distribution of 120 representative monomers. (b) Calculation of the mean bending persistence length by fitting all measured data. (c) AFM images and extracted monomer contours shown in (a). 
a

4HB-5nt-100\%

Number of samples $=660$

Mean bending persistence length $=300 \mathrm{~nm}$

Stdev, bending persistence length $=10 \mathrm{~nm}$

$R^{2}=0.9925$

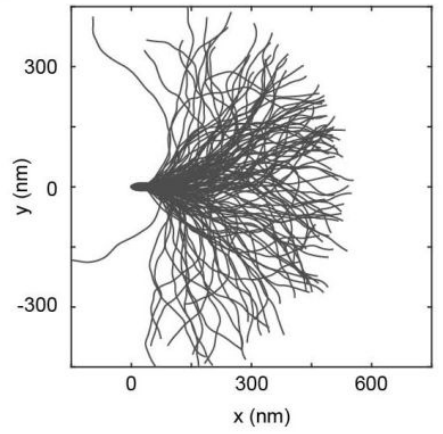

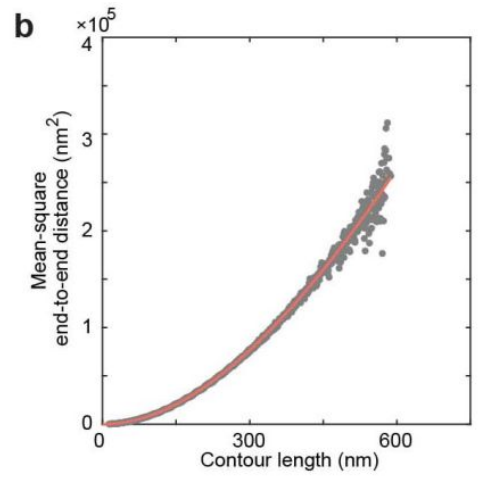

C

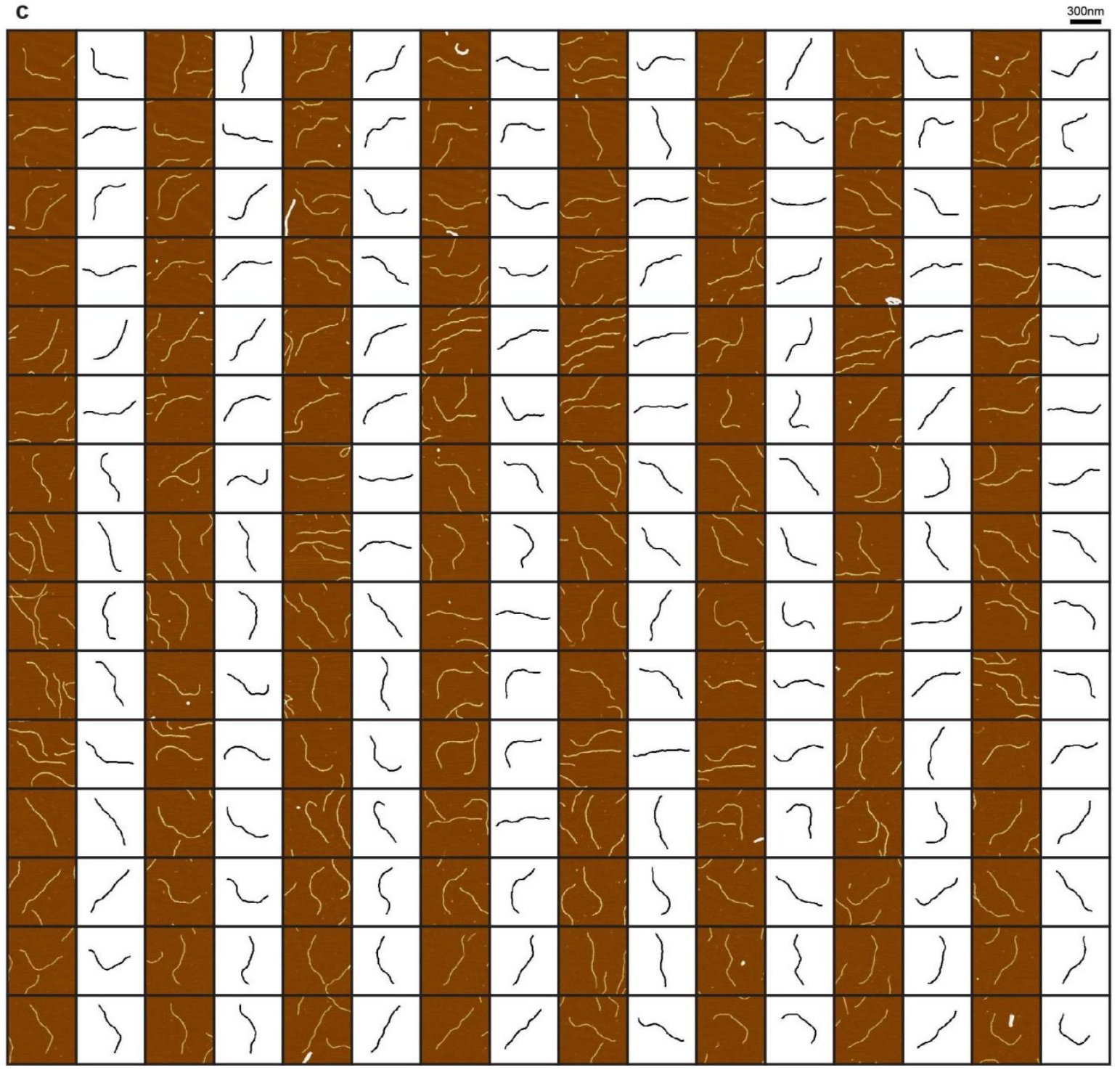

Supplementary Fig. 15. Experimental characterization of 4HB-5nt-100\% design. (a) Aligned contour distribution of 120 representative monomers. (b) Calculation of the mean bending persistence length by fitting all measured data. (c) AFM images and extracted monomer contours shown in (a). 
2-3. Experimental characterization of $6 \mathrm{HB}$ designs
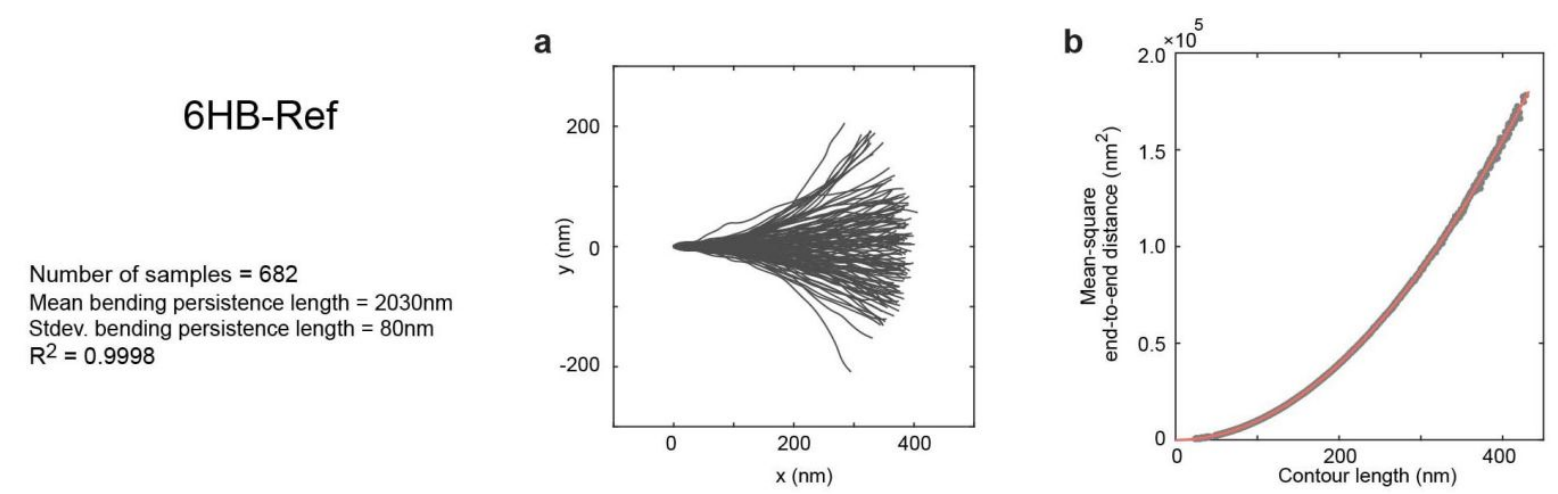

c

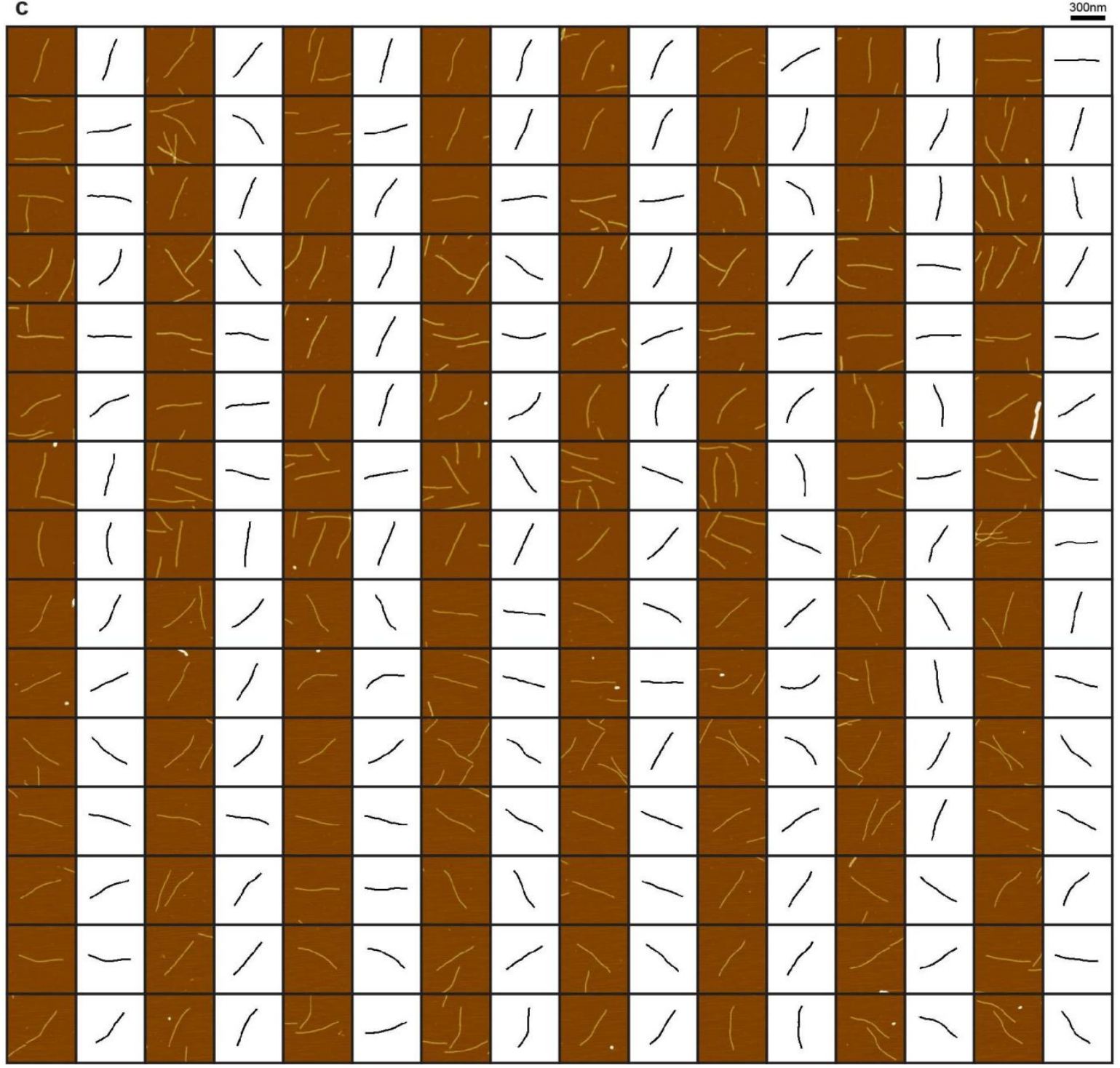

Supplementary Fig. 16. Experimental characterization of 6HB-Ref design. (a) Aligned contour distribution of 120 representative monomers. (b) Calculation of the mean bending persistence length by fitting all measured data. (c) AFM images and extracted monomer contours shown in (a). 
a

$6 \mathrm{HB}-1 \mathrm{nt}-17 \%$

Number of samples $=702$

Mean bending persistence length $=1830 \mathrm{~nm}$

Stdev. bending persistence length $=70 \mathrm{~nm}$

$R^{2}=0.9997$
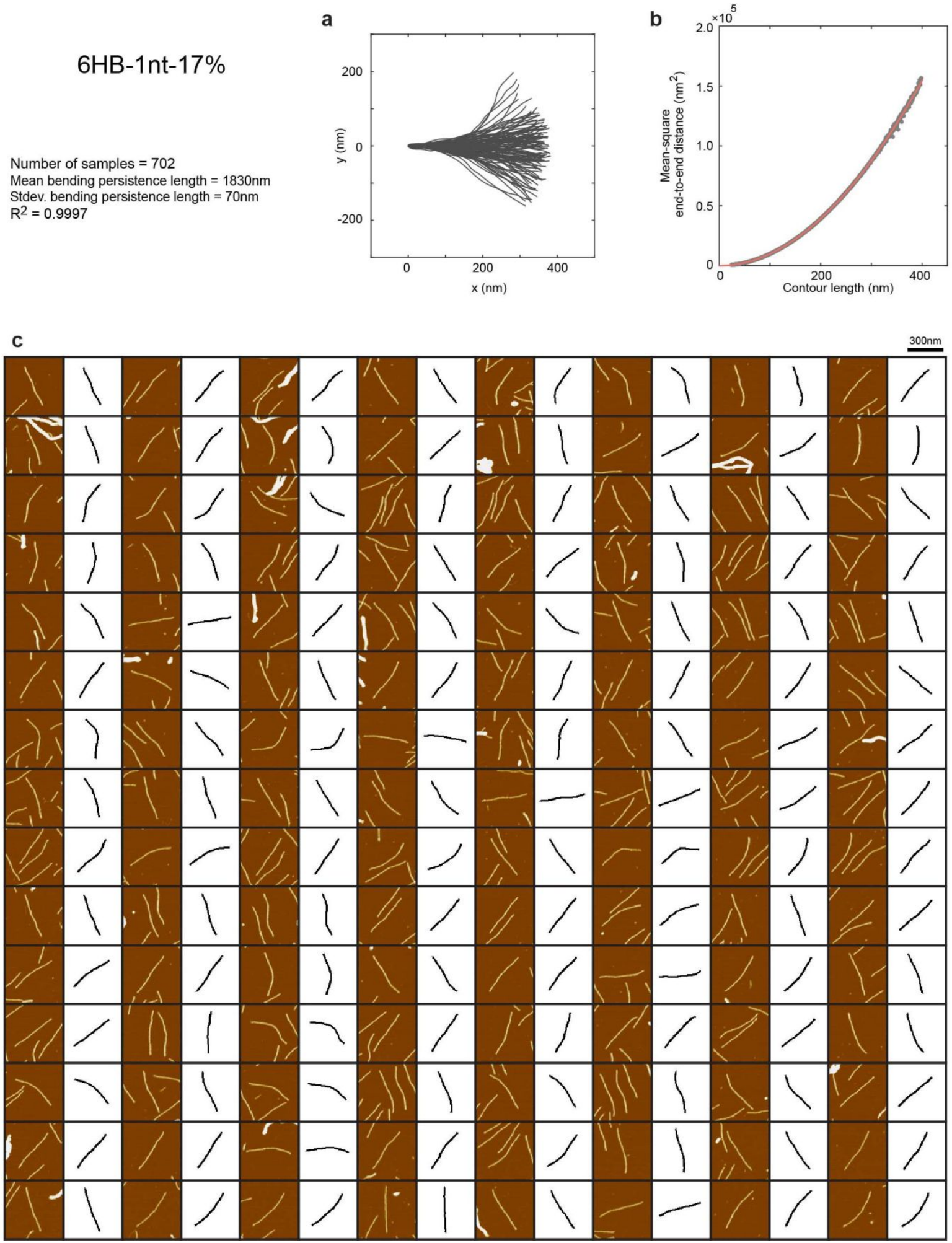

Supplementary Fig. 17. Experimental characterization of $6 \mathrm{HB}-1 \mathrm{nt}-17 \%$ design. (a) Aligned contour distribution of 120 representative monomers. (b) Calculation of the mean bending persistence length by fitting all measured data. (c) AFM images and extracted monomer contours shown in (a). 

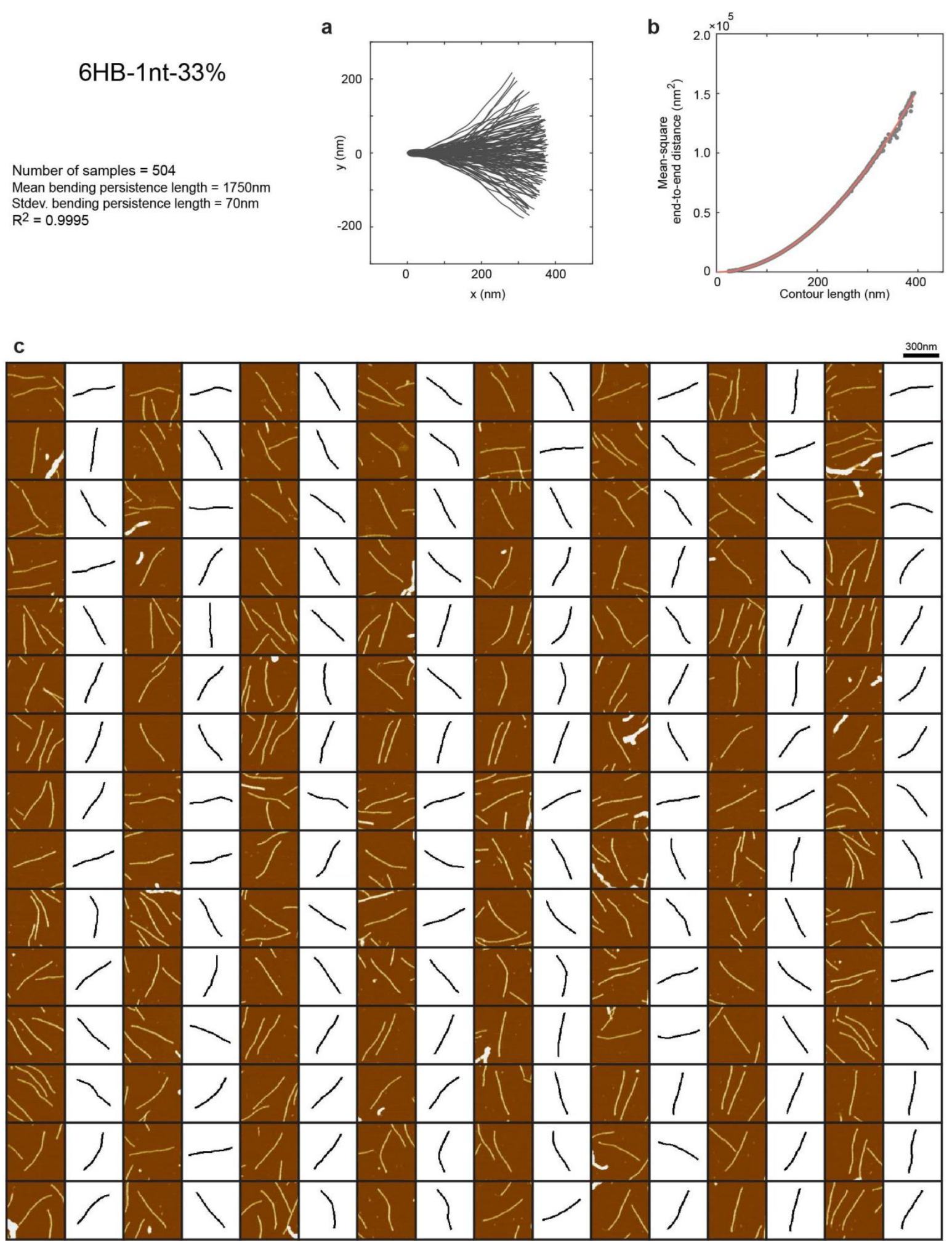

Supplementary Fig. 18. Experimental characterization of $6 \mathrm{HB}-1 \mathrm{nt}-33 \%$ design. (a) Aligned contour distribution of 120 representative monomers. (b) Calculation of the mean bending persistence length by fitting all measured data. (c) AFM images and extracted monomer contours shown in (a). 

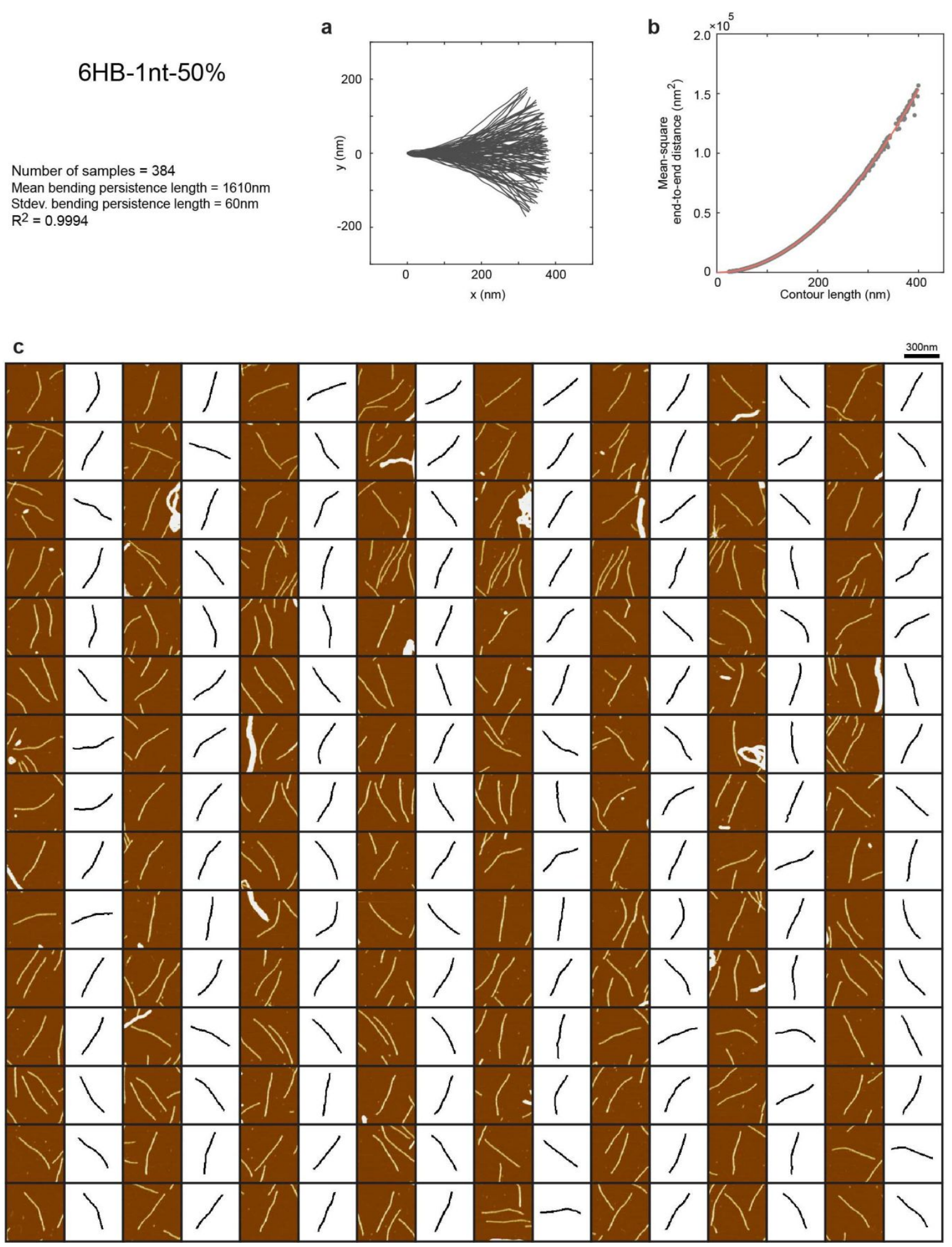

Supplementary Fig. 19. Experimental characterization of $6 \mathrm{HB}-1 \mathrm{nt}-50 \%$ design. (a) Aligned contour distribution of 120 representative monomers. (b) Calculation of the mean bending persistence length by fitting all measured data. (c) AFM images and extracted monomer contours shown in (a). 
a

6 HB-1nt-67\%

Number of samples $=647$

Mean bending persistence length $=1540 \mathrm{~nm}$

Stdev. bending persistence length $=60 \mathrm{~nm}$

$R^{2}=0.9996$
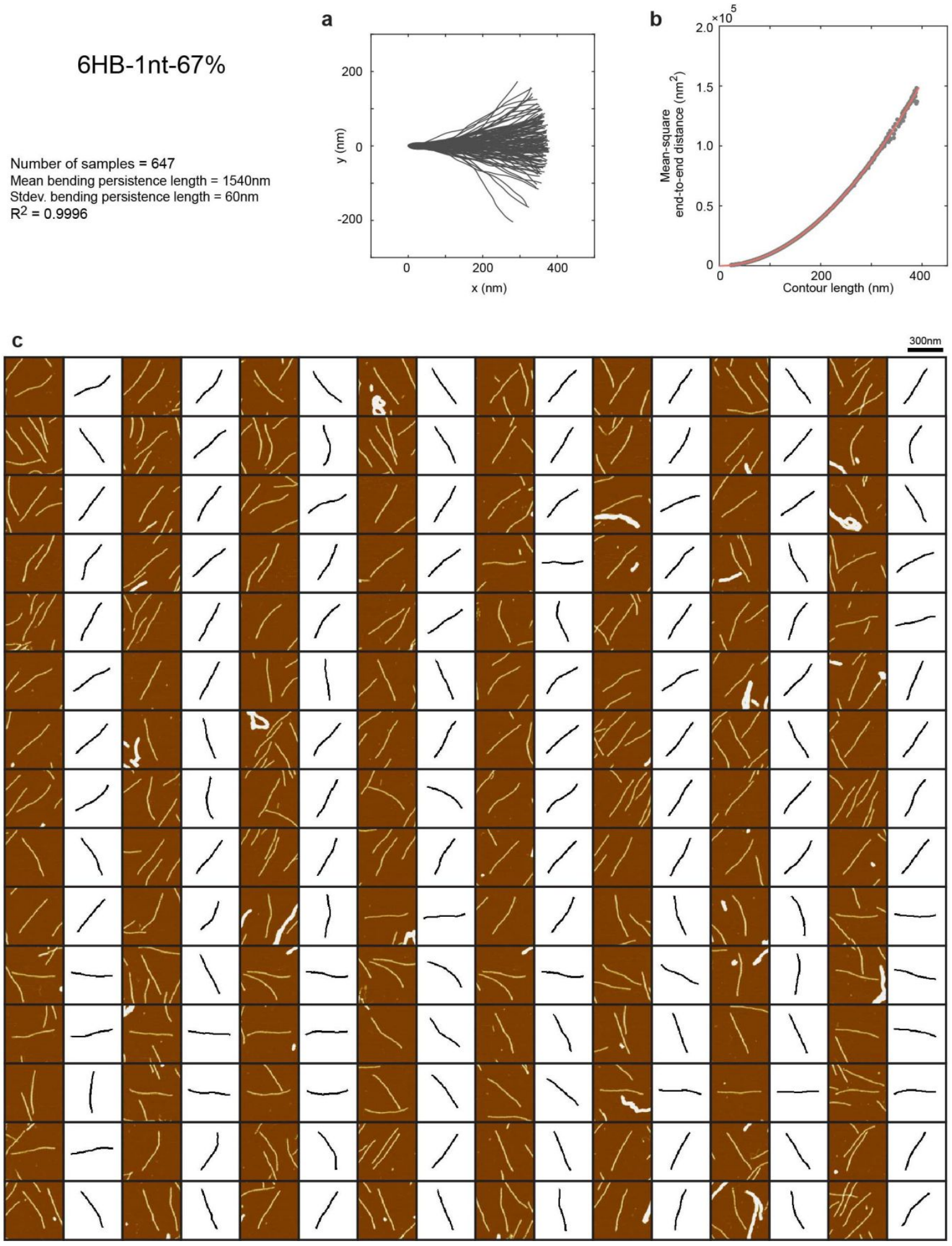

Supplementary Fig. 20. Experimental characterization of $6 \mathrm{HB}-1 \mathrm{nt}-67 \%$ design. (a) Aligned contour distribution of 120 representative monomers. (b) Calculation of the mean bending persistence length by fitting all measured data. (c) AFM images and extracted monomer contours shown in (a). 

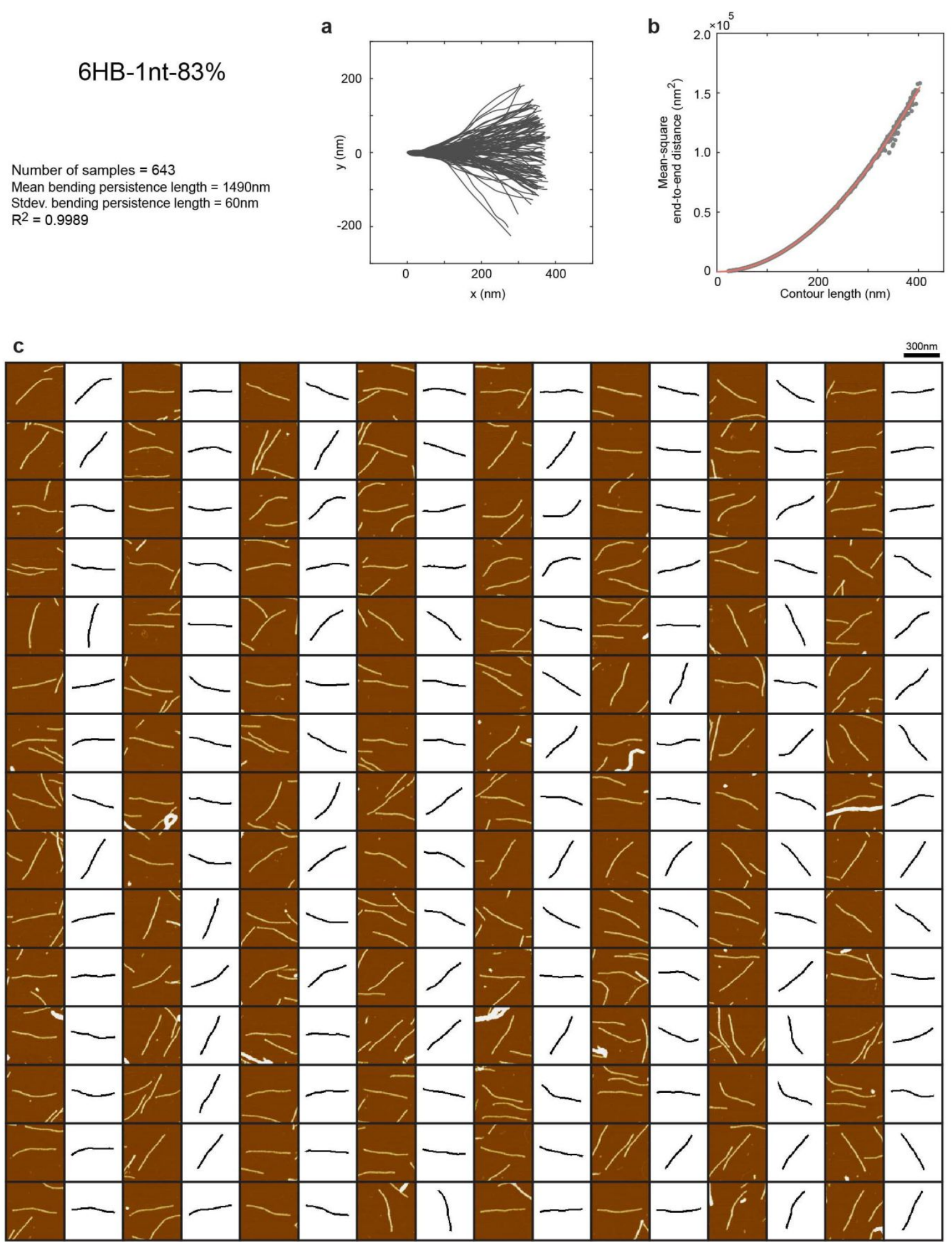

Supplementary Fig. 21. Experimental characterization of $6 \mathrm{HB}-1 \mathrm{nt}-83 \%$ design. (a) Aligned contour distribution of 120 representative monomers. (b) Calculation of the mean bending persistence length by fitting all measured data. (c) AFM images and extracted monomer contours shown in (a). 
a

$6 \mathrm{HB}-1 \mathrm{nt}-100 \%$

Number of samples $=674$

Mean bending persistence length $=1450 \mathrm{~nm}$

Stdev. bending persistence length $=90 \mathrm{~nm}$

$R^{2}=0.9989$
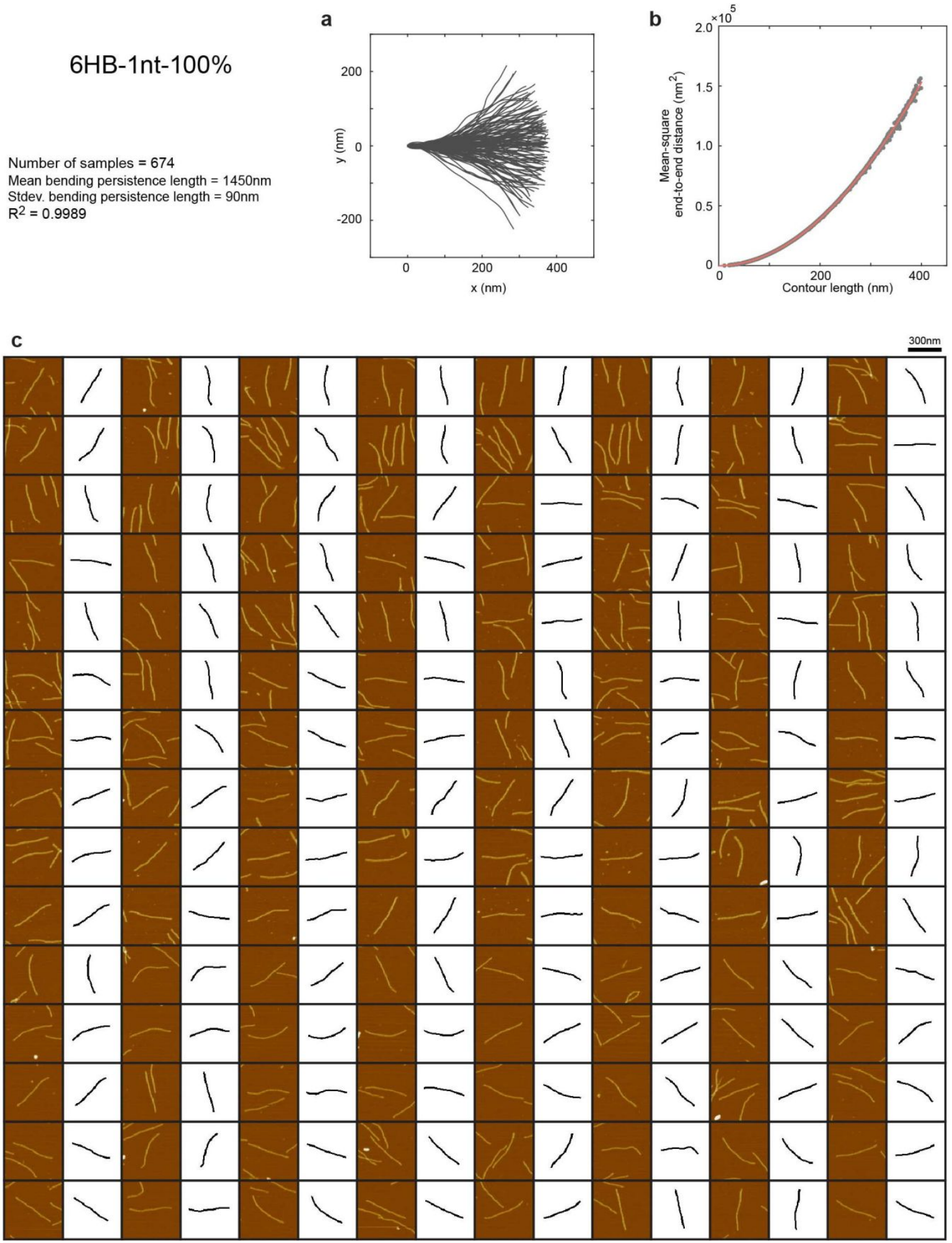

Supplementary Fig. 22. Experimental characterization of 6HB-1nt-100\% design. (a) Aligned contour distribution of 120 representative monomers. (b) Calculation of the mean bending persistence length by fitting all measured data. (c) AFM images and extracted monomer contours shown in (a). 
a

6 HB-2nt-100\%

Number of samples $=627$

Mean bending persistence length $=1040 \mathrm{~nm}$

Stdev . bending persistence length $=40 \mathrm{~nm}$

$R^{2}=0.9989$
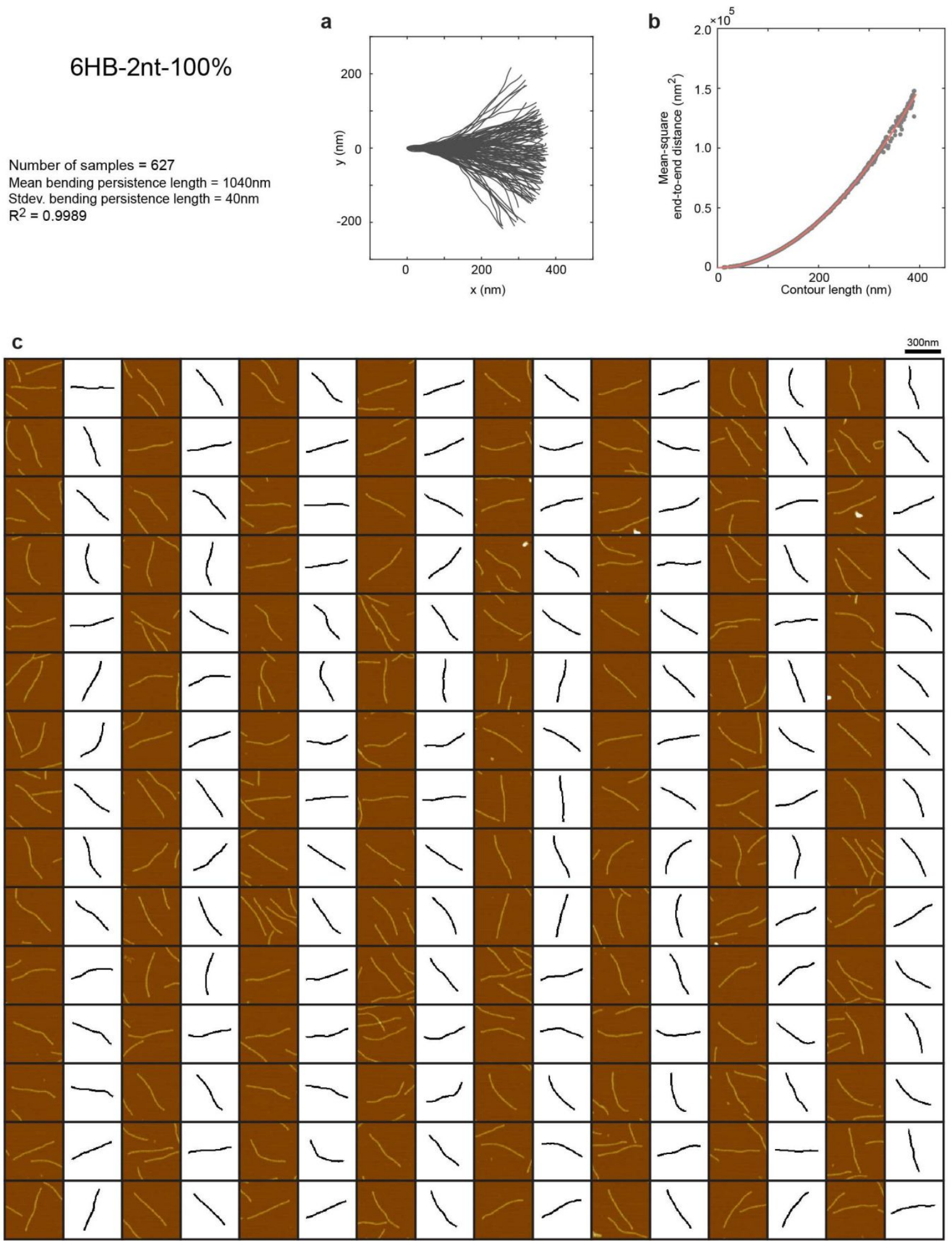

Supplementary Fig. 23. Experimental characterization of 6HB-2nt-100\% design. (a) Aligned contour distribution of 120 representative monomers. (b) Calculation of the mean bending persistence length by fitting all measured data. (c) AFM images and extracted monomer contours shown in (a). 
a

6 HB-3nt-17\%

Number of samples $=442$

Mean bending persistence length $=1780 \mathrm{~nm}$

Stdev. bending persistence length $=70 \mathrm{~nm}$

$R^{2}=0.9995$
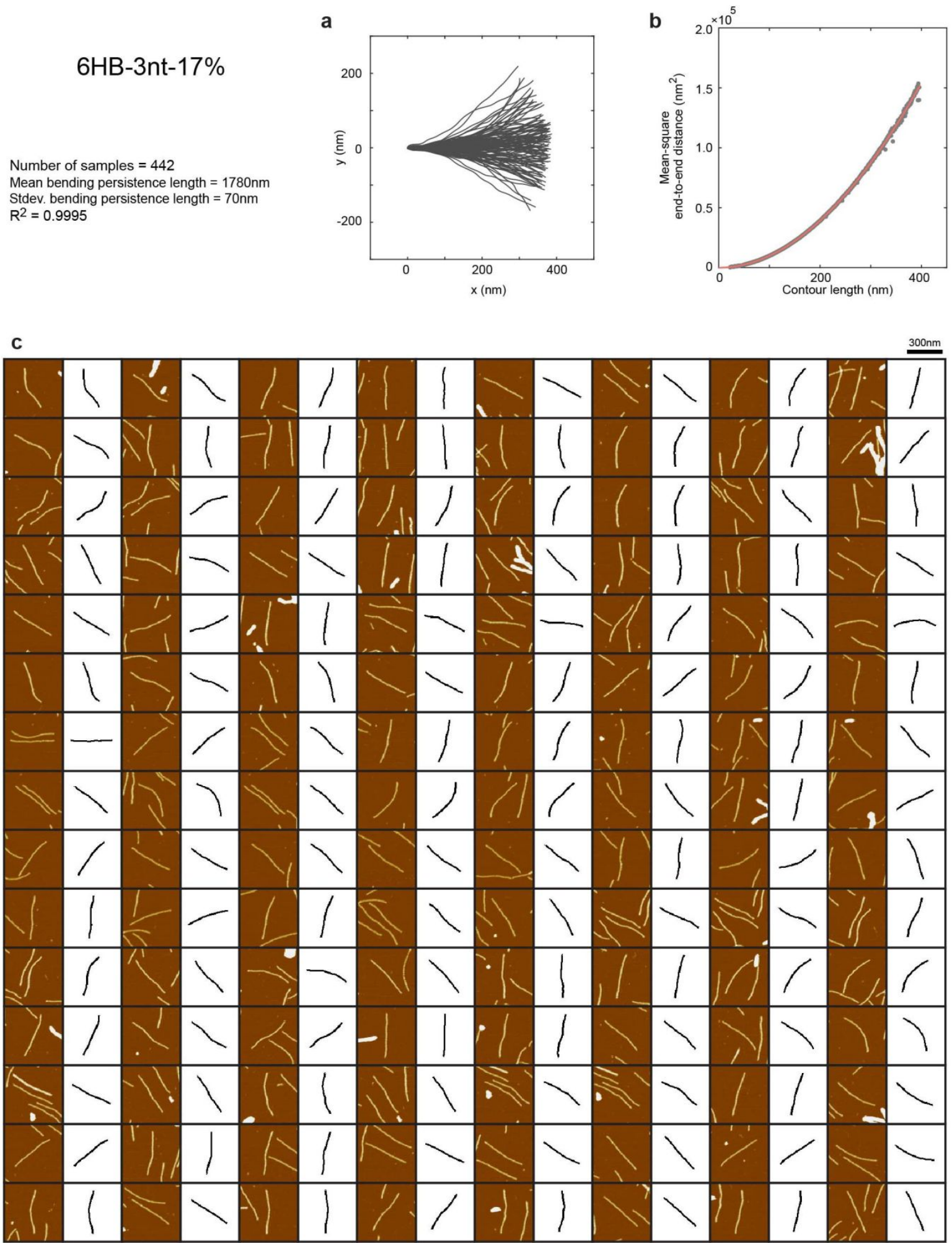

Supplementary Fig. 24. Experimental characterization of $6 \mathrm{HB}-3 \mathrm{nt}-17 \%$ design. (a) Aligned contour distribution of 120 representative monomers. (b) Calculation of the mean bending persistence length by fitting all measured data. (c) AFM images and extracted monomer contours shown in (a). 

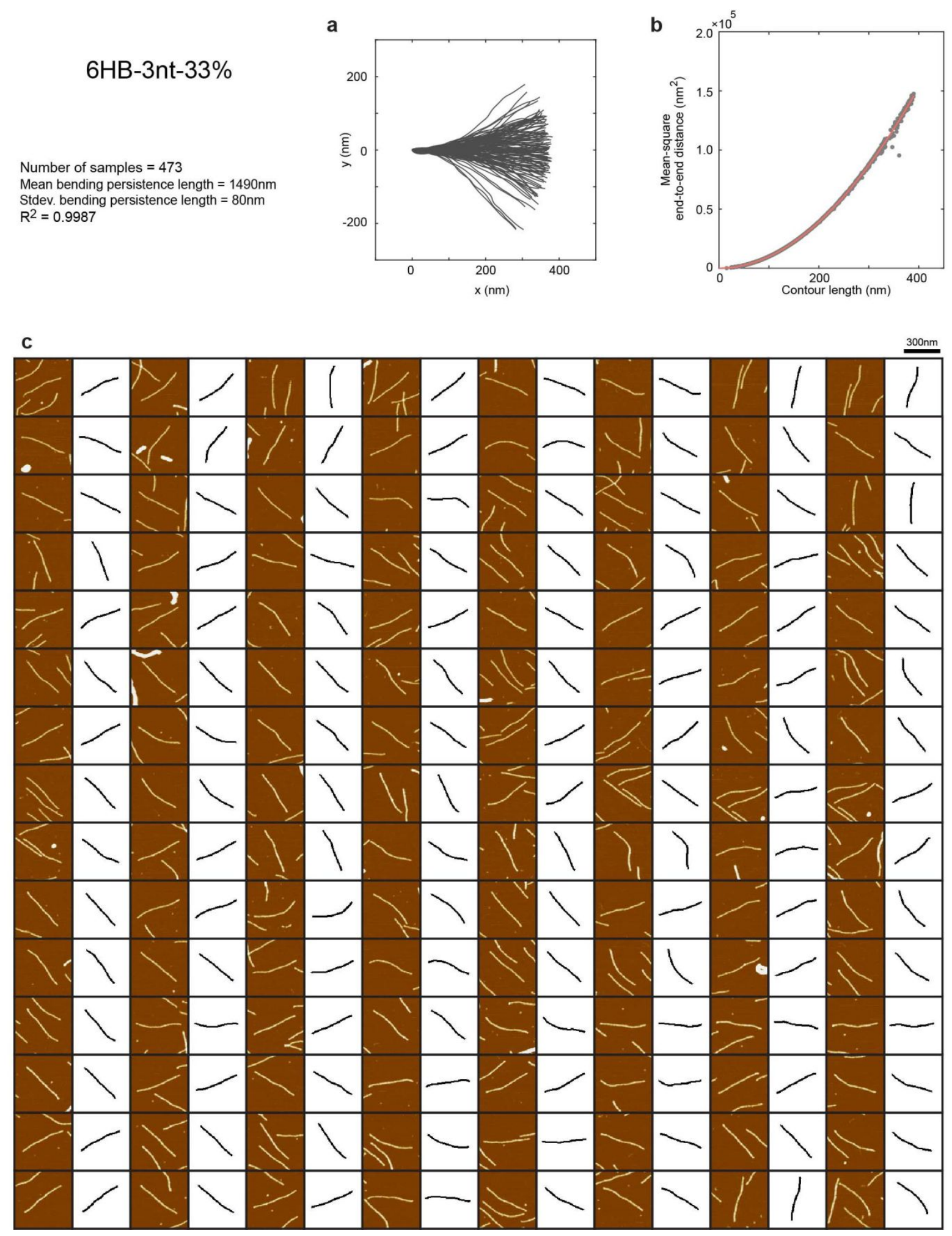

Supplementary Fig. 25. Experimental characterization of $6 \mathrm{HB}-3 \mathrm{nt}-33 \%$ design. (a) Aligned contour distribution of 120 representative monomers. (b) Calculation of the mean bending persistence length by fitting all measured data. (c) AFM images and extracted monomer contours shown in (a). 

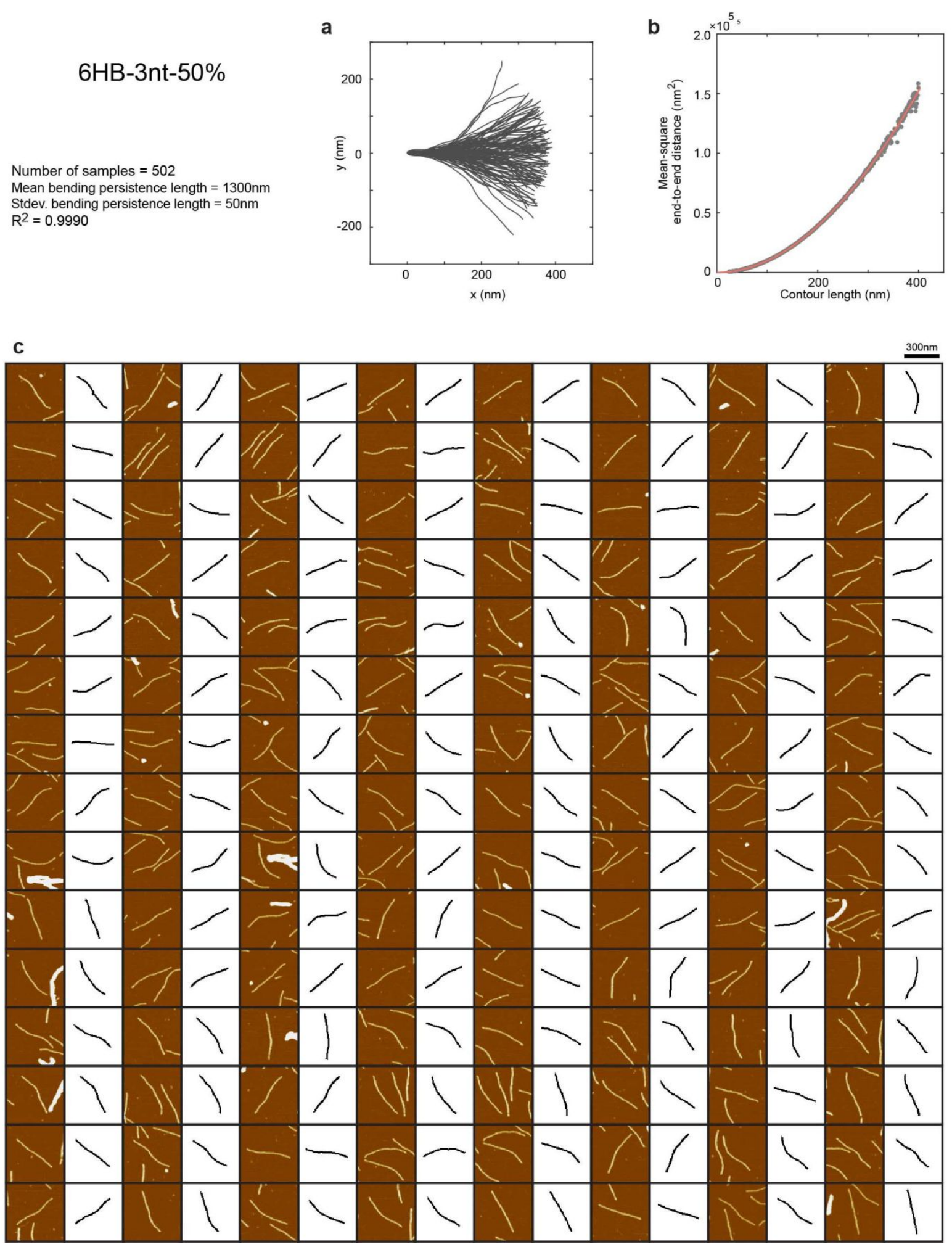

Supplementary Fig. 26. Experimental characterization of $6 \mathrm{HB}-3 \mathrm{nt}-50 \%$ design. (a) Aligned contour distribution of 120 representative monomers. (b) Calculation of the mean bending persistence length by fitting all measured data. (c) AFM images and extracted monomer contours shown in (a). 
a

6 HB-3nt-67\%

Number of samples $=553$

Mean bending persistence length $=1170 \mathrm{~nm}$

Stdev. bending persistence length $=60 \mathrm{~nm}$

$R^{2}=0.9988$
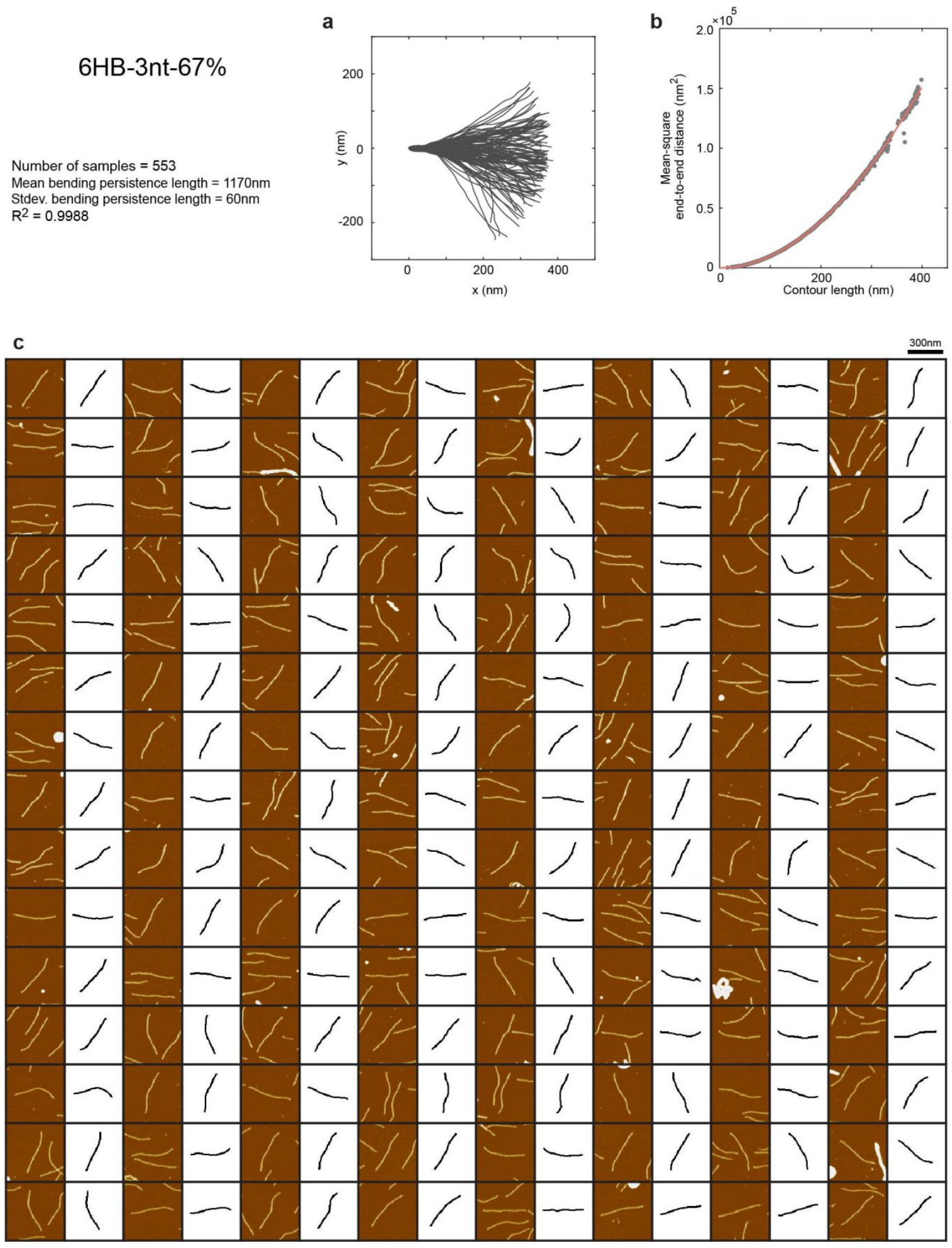

Supplementary Fig. 27. Experimental characterization of $6 \mathrm{HB}-3 \mathrm{nt}-67 \%$ design. (a) Aligned contour distribution of 120 representative monomers. (b) Calculation of the mean bending persistence length by fitting all measured data. (c) AFM images and extracted monomer contours shown in (a). 

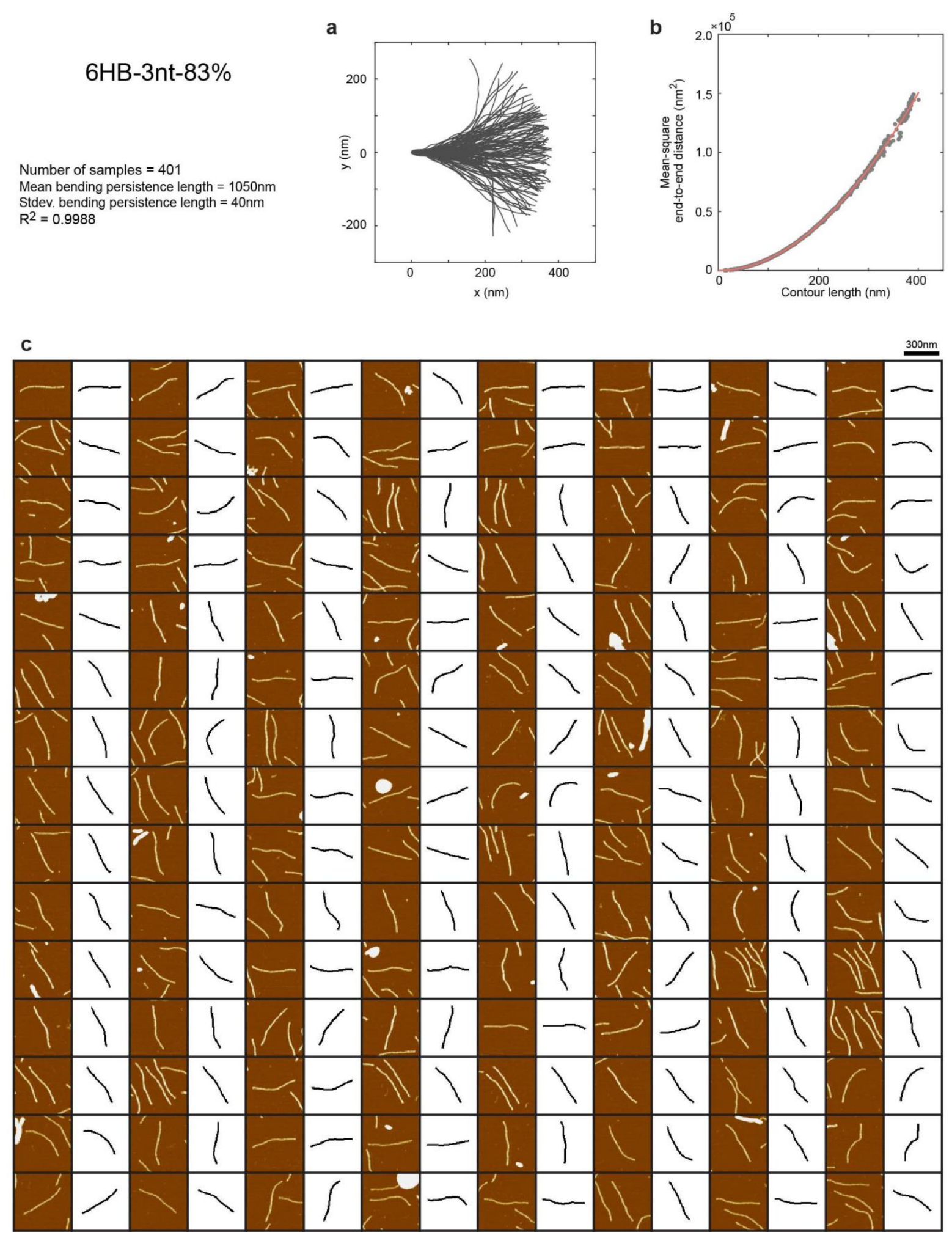

Supplementary Fig. 28. Experimental characterization of $6 \mathrm{HB}-3 \mathrm{nt}-83 \%$ design. (a) Aligned contour distribution of 120 representative monomers. (b) Calculation of the mean bending persistence length by fitting all measured data. (c) AFM images and extracted monomer contours shown in (a). 
a

6 HB-3nt-100\%

Number of samples $=438$

Mean bending persistence length $=870 \mathrm{~nm}$

Stdev. bending persistence length $=30 \mathrm{~nm}$

$R^{2}=0.9971$
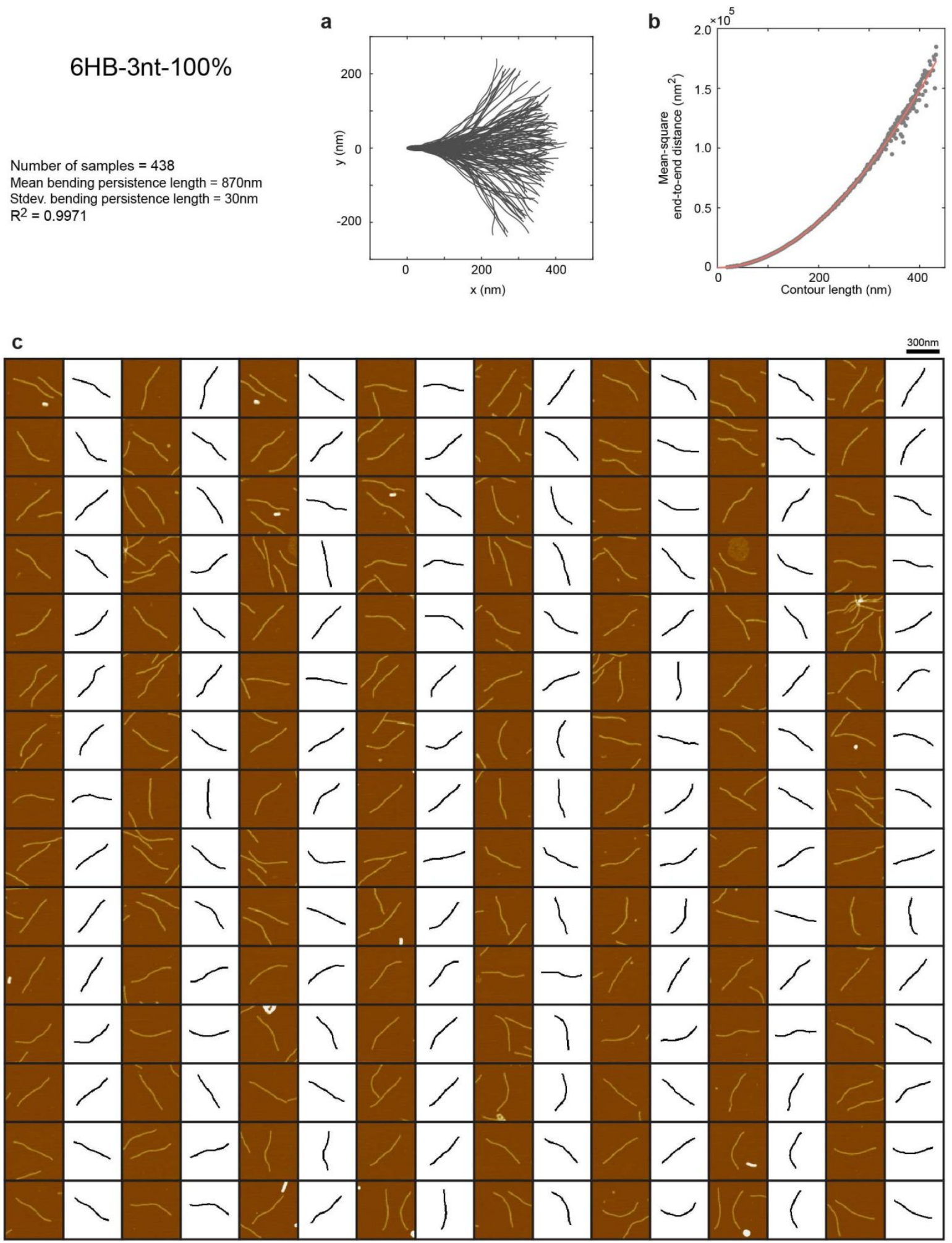

Supplementary Fig. 29. Experimental characterization of 6HB-3nt-100\% design. (a) Aligned contour distribution of 120 representative monomers. (b) Calculation of the mean bending persistence length by fitting all measured data. (c) AFM images and extracted monomer contours shown in (a). 
a

6 HB-4nt-100\%

Number of samples $=418$

Mean bending persistence length $=750 \mathrm{~nm}$

Stdev. bending persistence length $=30 \mathrm{~nm}$

$\mathrm{R}^{2}=0.9967$
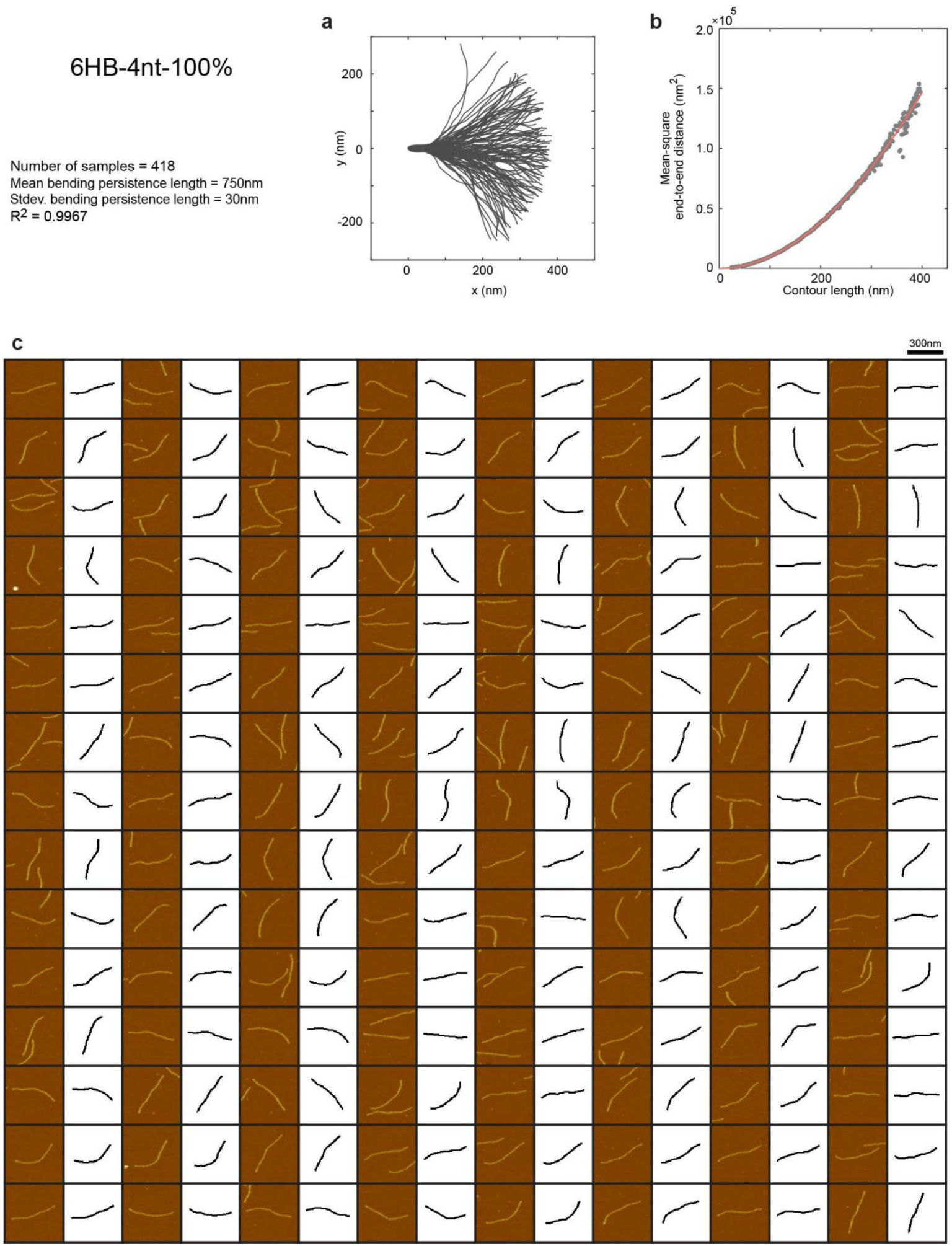

Supplementary Fig. 30. Experimental characterization of 6HB-4nt-100\% design. (a) Aligned contour distribution of 120 representative monomers. (b) Calculation of the mean bending persistence length by fitting all measured data. (c) AFM images and extracted monomer contours shown in (a). 

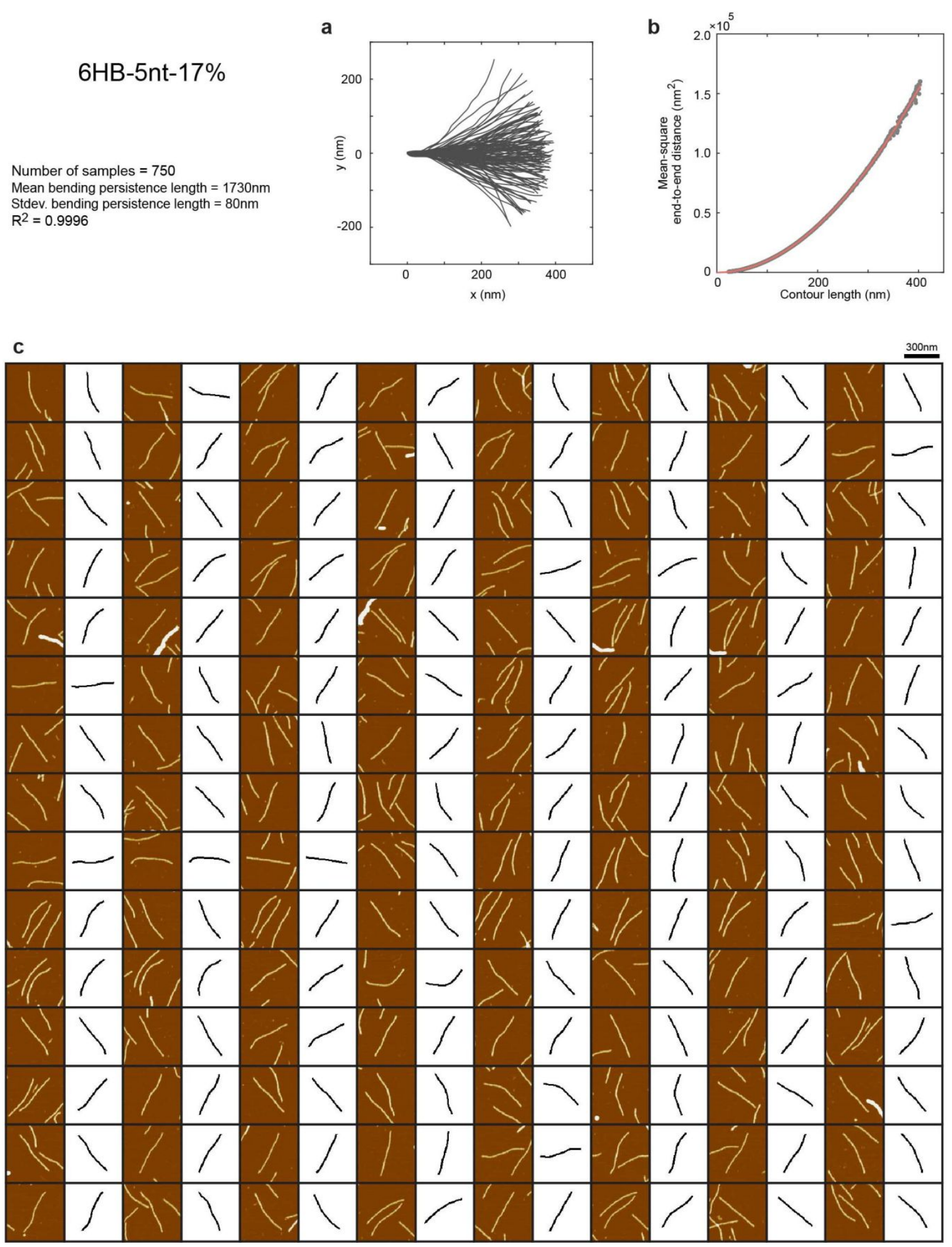

Supplementary Fig. 31. Experimental characterization of $6 \mathrm{HB}-5 \mathrm{nt}-17 \%$ design. (a) Aligned contour distribution of 120 representative monomers. (b) Calculation of the mean bending persistence length by fitting all measured data. (c) AFM images and extracted monomer contours shown in (a). 
a

6HB-5nt-33\%

Number of samples $=394$

Mean bending persistence length $=1420 \mathrm{~nm}$

Stdev. bending persistence length $=60 \mathrm{~nm}$

$R^{2}=0.9990$
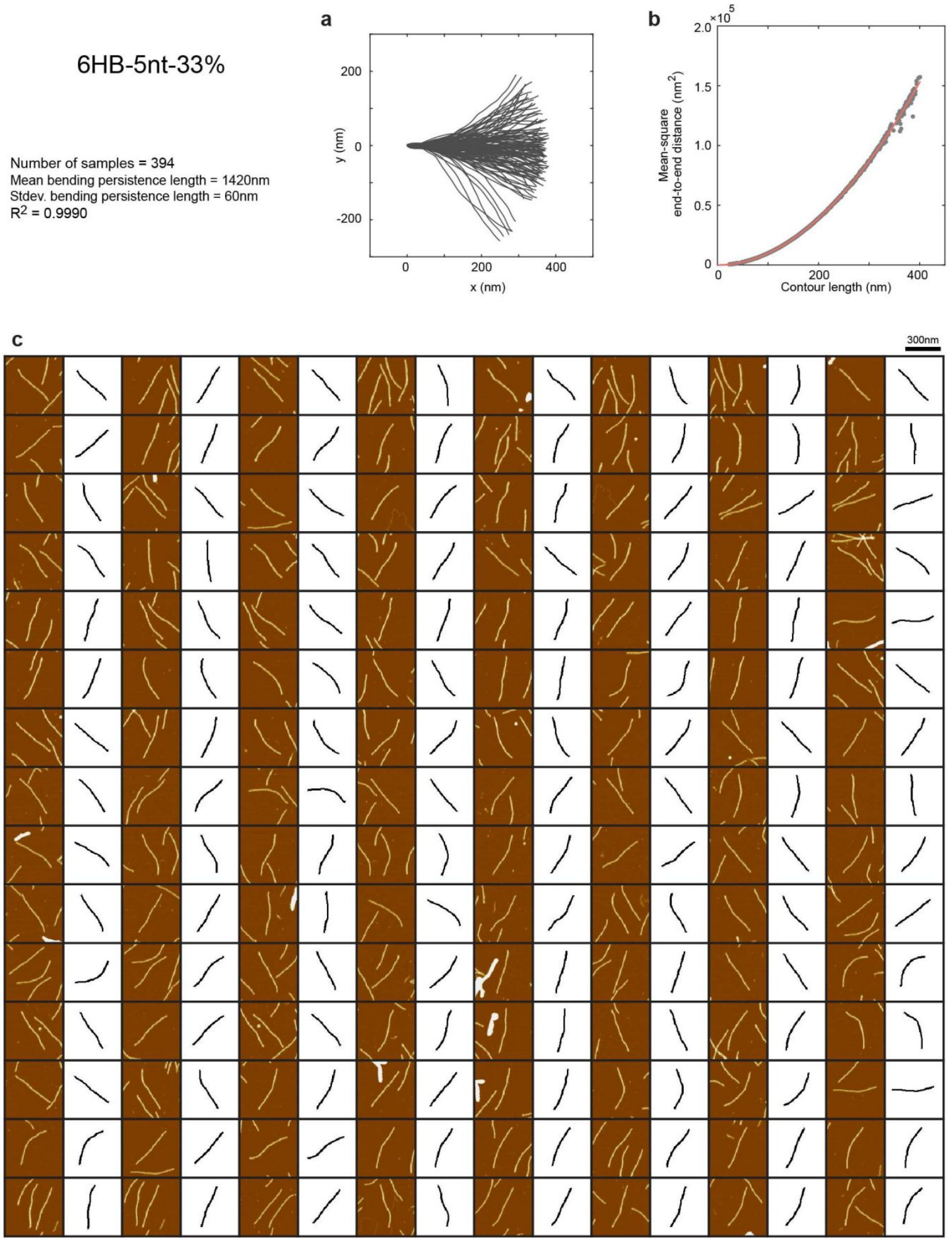

Supplementary Fig. 32. Experimental characterization of $6 \mathrm{HB}-5 \mathrm{nt}-33 \%$ design. (a) Aligned contour distribution of 120 representative monomers. (b) Calculation of the mean bending persistence length by fitting all measured data. (c) AFM images and extracted monomer contours shown in (a). 
a

6 HB-5nt-50\%

Number of samples $=665$

Mean bending persistence length $=1190 \mathrm{~nm}$

Stdev. bending persistence length $=50 \mathrm{~nm}$

$R^{2}=0.9993$
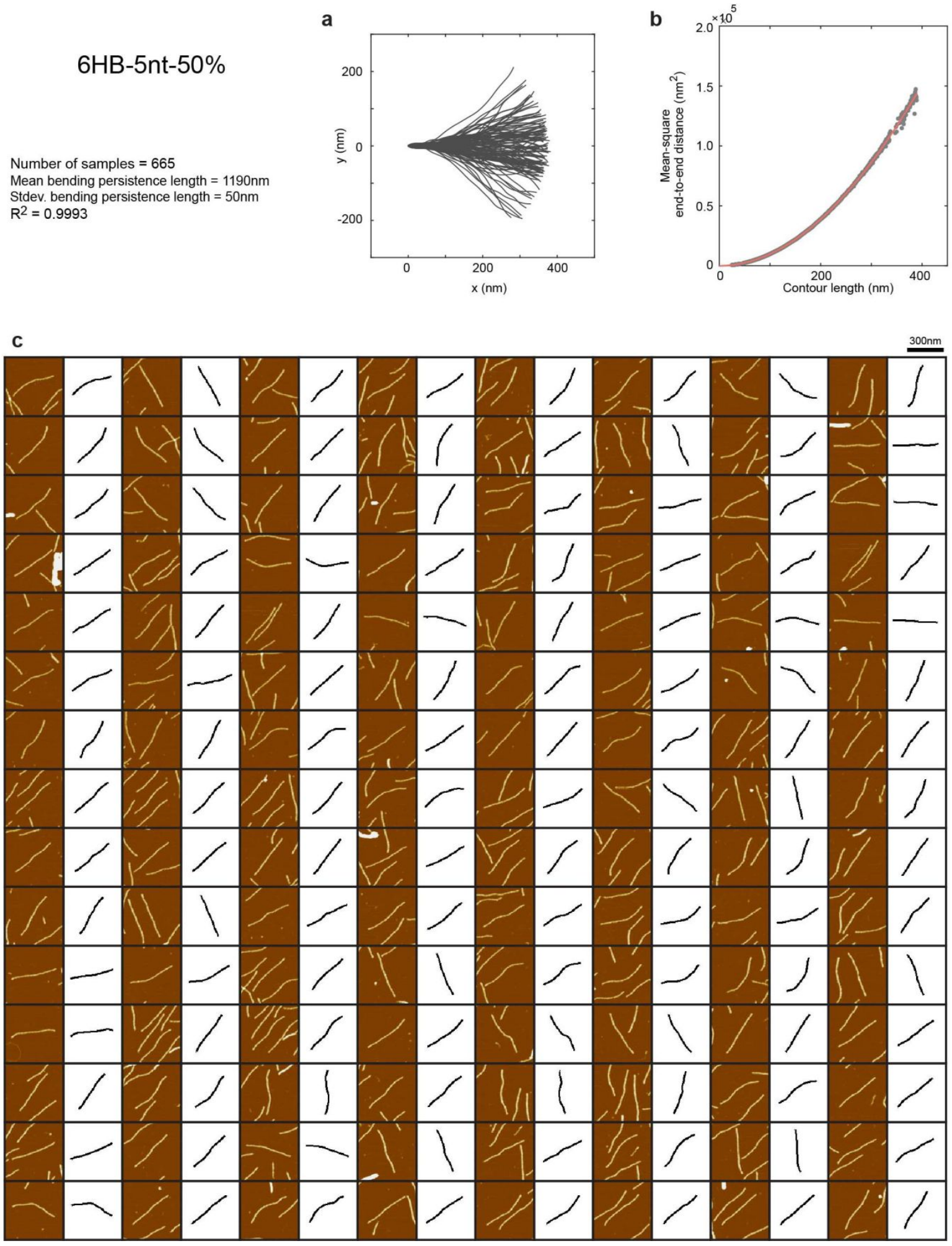

Supplementary Fig. 33. Experimental characterization of $6 \mathrm{HB}-5 \mathrm{nt}-50 \%$ design. (a) Aligned contour distribution of 120 representative monomers. (b) Calculation of the mean bending persistence length by fitting all measured data. (c) AFM images and extracted monomer contours shown in (a). 

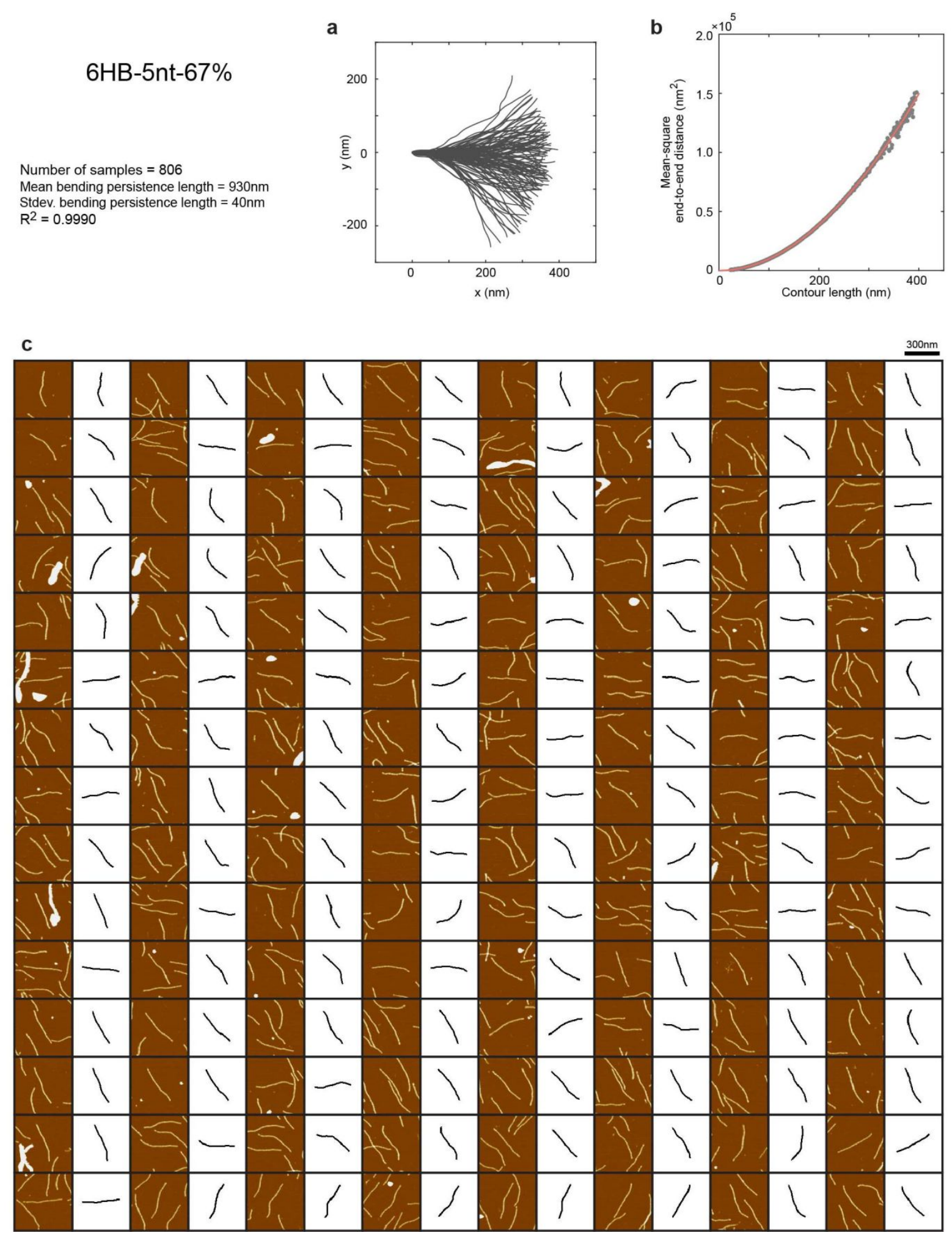

Supplementary Fig. 34. Experimental characterization of 6HB-5nt-67\% design. (a) Aligned contour distribution of 120 representative monomers. (b) Calculation of the mean bending persistence length by fitting all measured data. (c) AFM images and extracted monomer contours shown in (a). 
a

6 HB-5nt-83\%

Number of samples $=1073$

Mean bending persistence length $=820 \mathrm{~nm}$

Stdev. bending persistence length $=40 \mathrm{~nm}$

$R^{2}=0.9973$
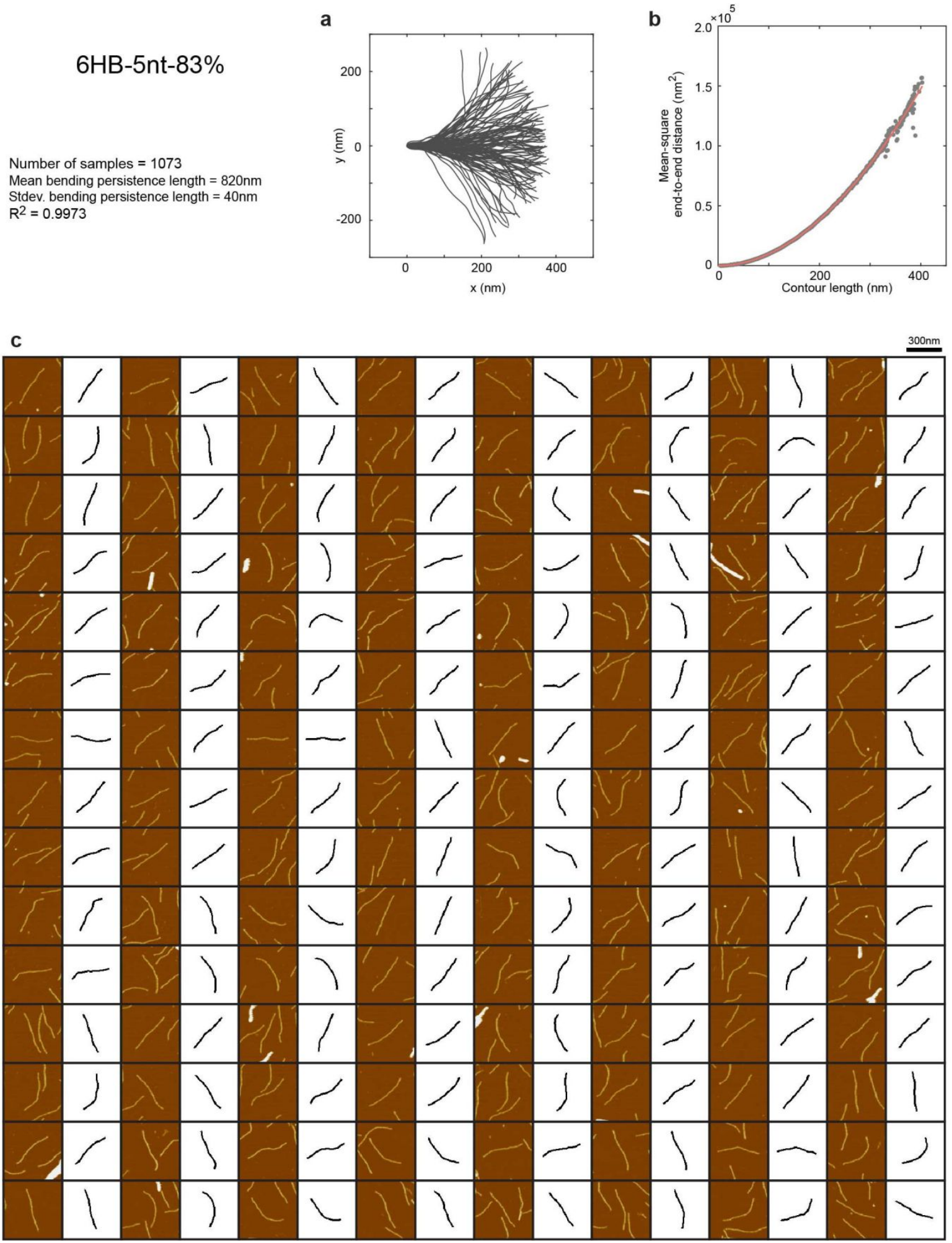

Supplementary Fig. 35. Experimental characterization of $6 \mathrm{HB}-5 \mathrm{nt}-83 \%$ design. (a) Aligned contour distribution of 120 representative monomers. (b) Calculation of the mean bending persistence length by fitting all measured data. (c) AFM images and extracted monomer contours shown in (a). 
a

6 HB-5nt-100\%

Number of samples $=779$

Mean bending persistence length $=660 \mathrm{~nm}$

Stdev. bending persistence length $=30 \mathrm{~nm}$

$\mathrm{R}^{2}=0.9972$
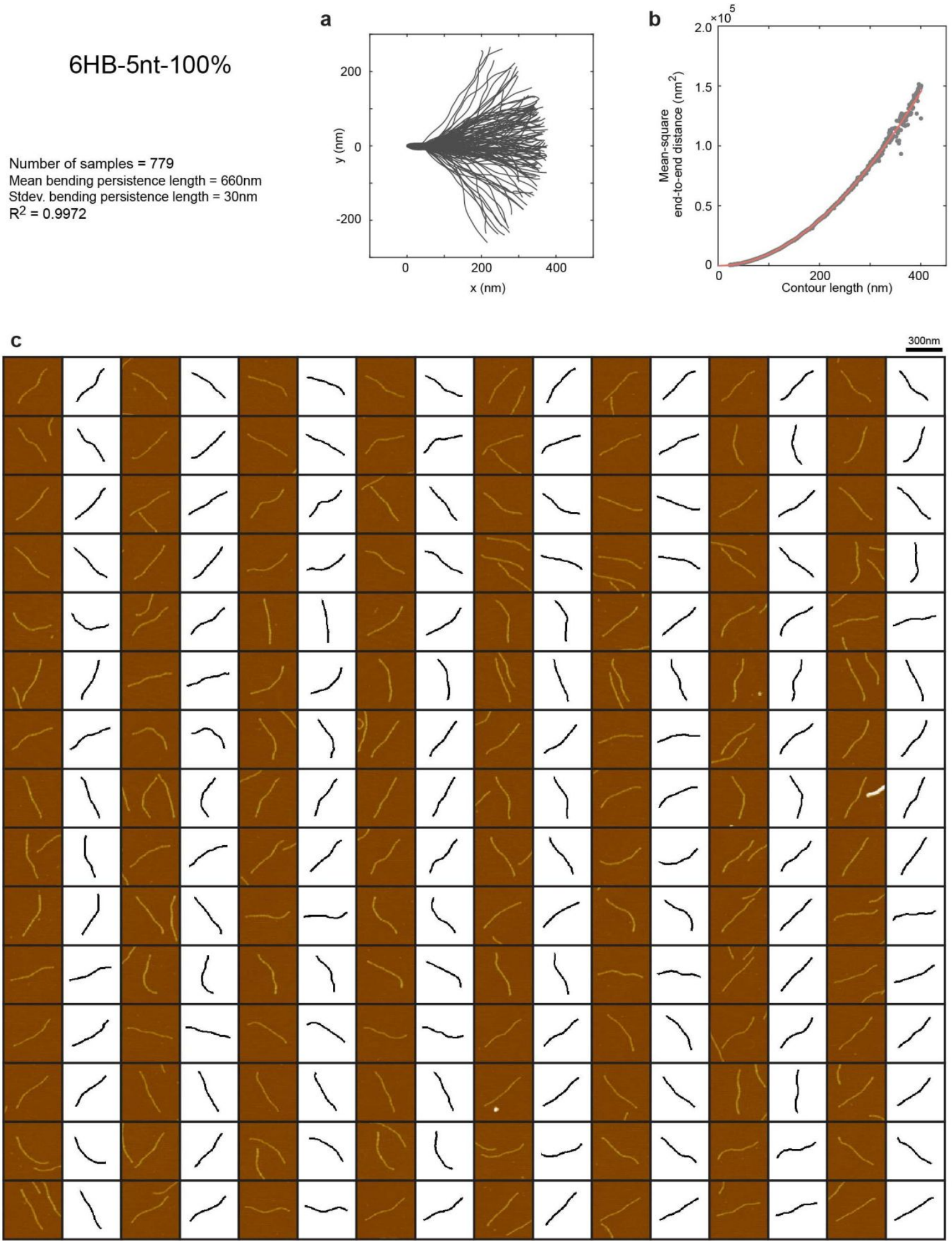

Supplementary Fig. 36. Experimental characterization of 6HB-5nt-100\% design. (a) Aligned contour distribution of 120 representative monomers. (b) Calculation of the mean bending persistence length by fitting all measured data. (c) AFM images and extracted monomer contours shown in (a). 


\section{Bending persistence length measurement from the monomer contour images}
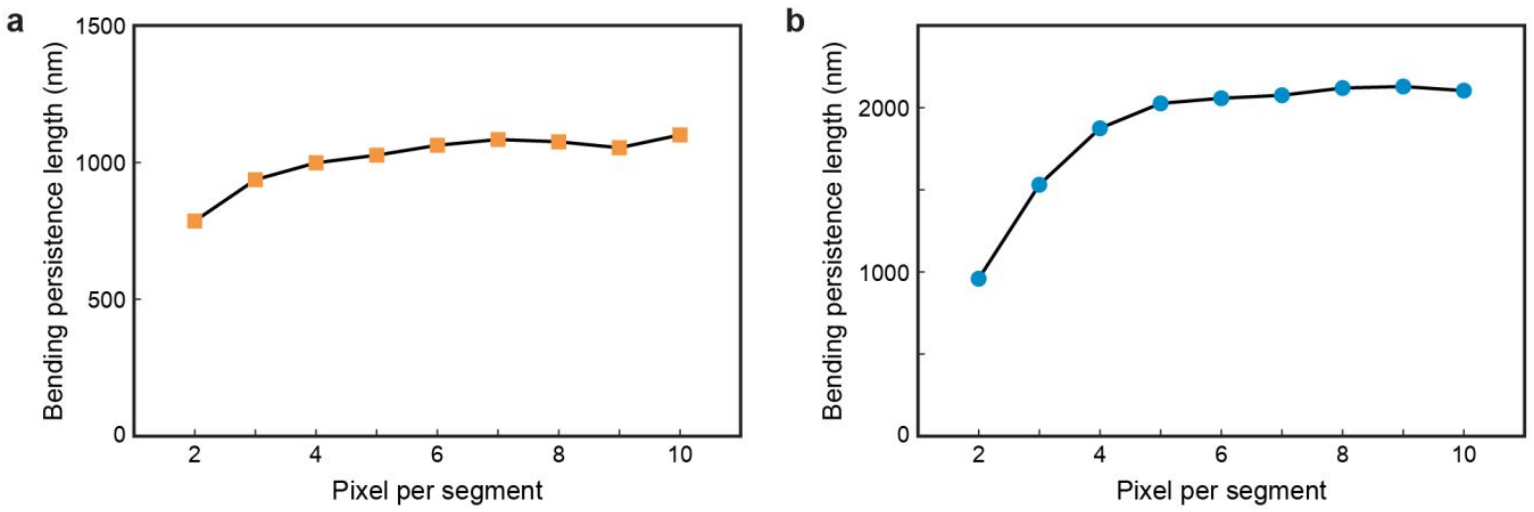

Supplementary Fig. 37. Bending persistence length measurement with different unit segment length. Bending persistence length of (a) 4HB-Ref and (b) 6HB-Ref design. Note that length of a unit segment is proportional to the value of pixel per segment. Resolution of a pixel is approximately 4.9 $\mathrm{nm} / \mathrm{px}$. 
a
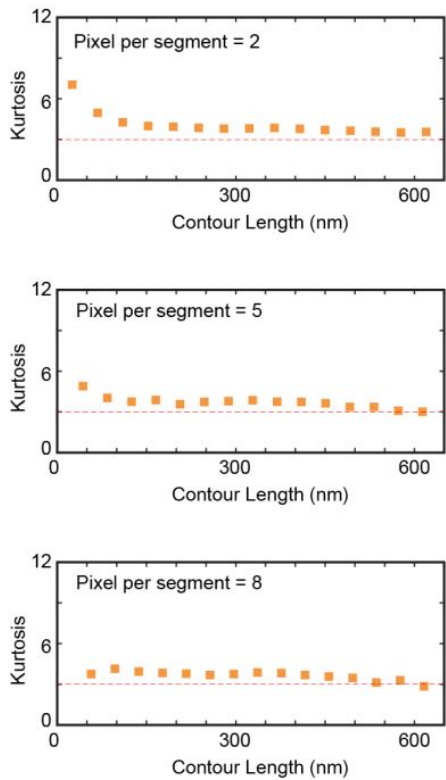

b
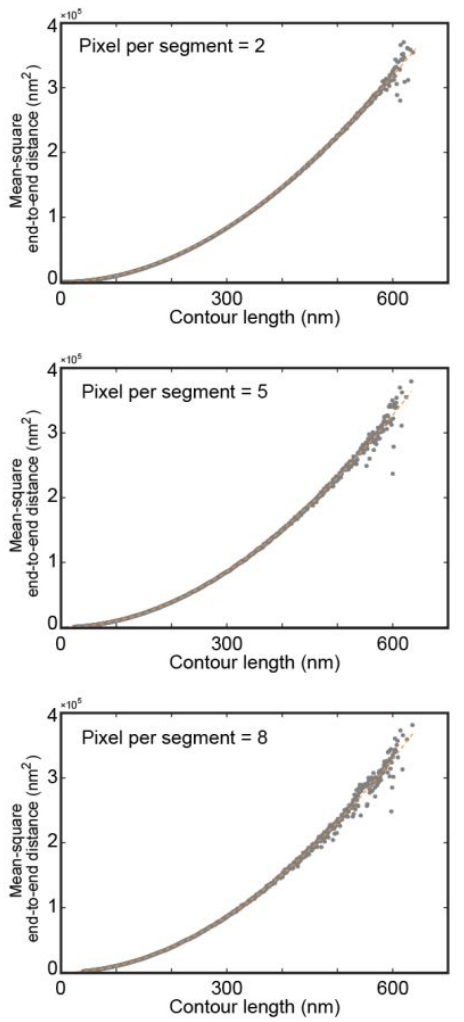
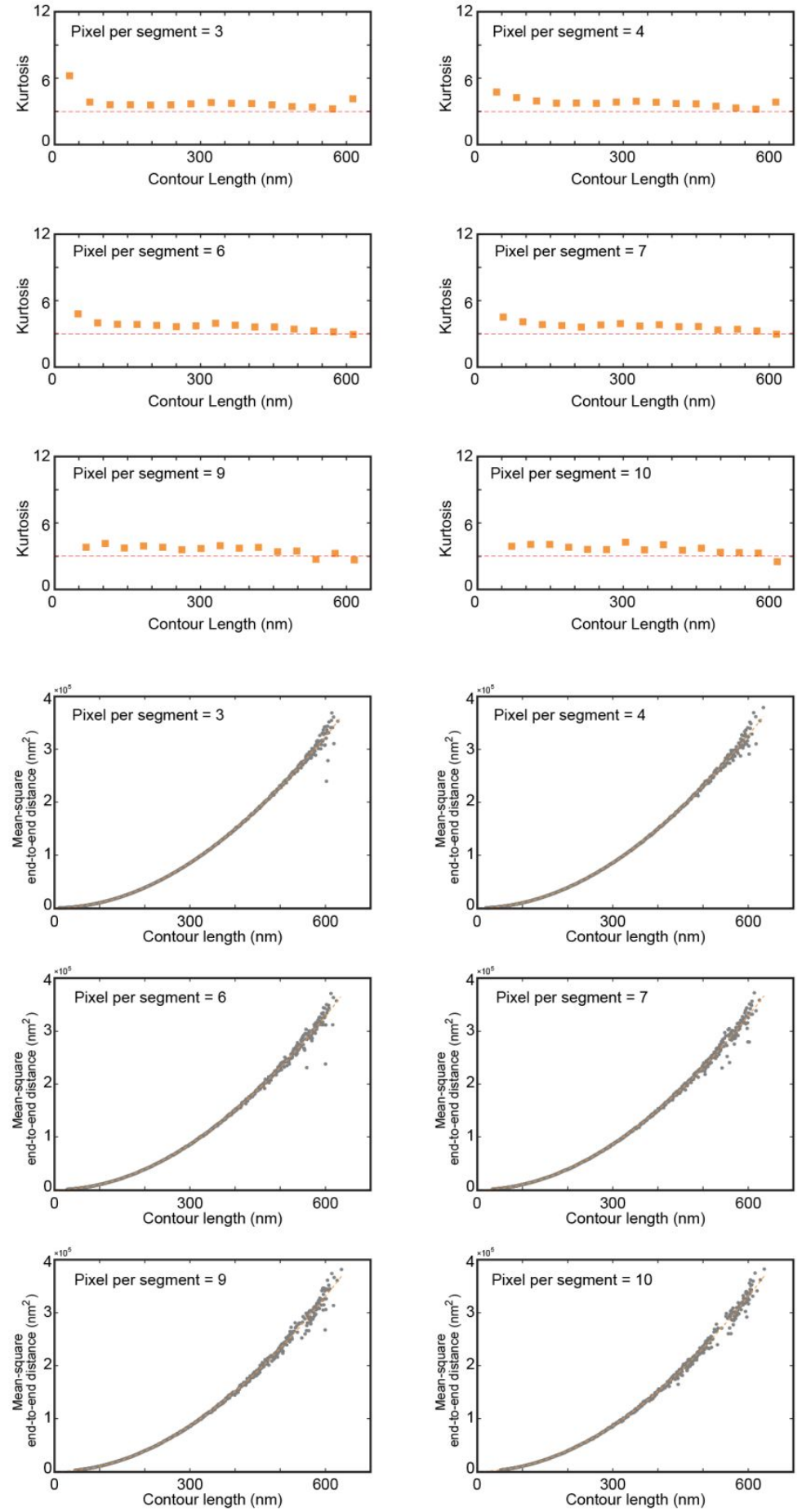

Supplementary Fig. 38. Parameter analysis on 4HB-Ref design. (a) Kurtosis analysis and (b) endto-end distance fitting curves. Calculated bending persistence length in each case was shown in Supplementary Fig. 37a. Pixel per segment value was chosen as 4 in the manuscript. 
a
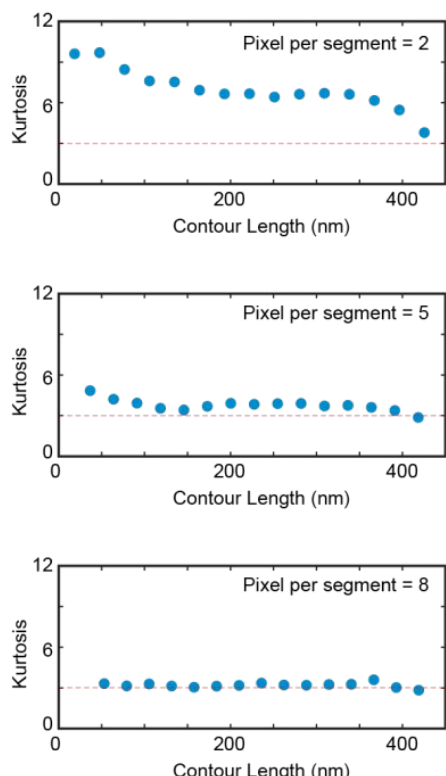

b
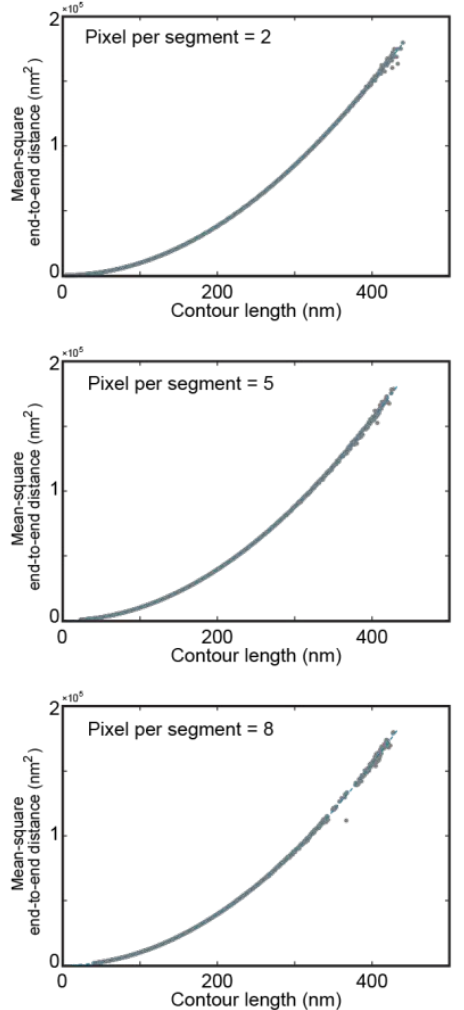
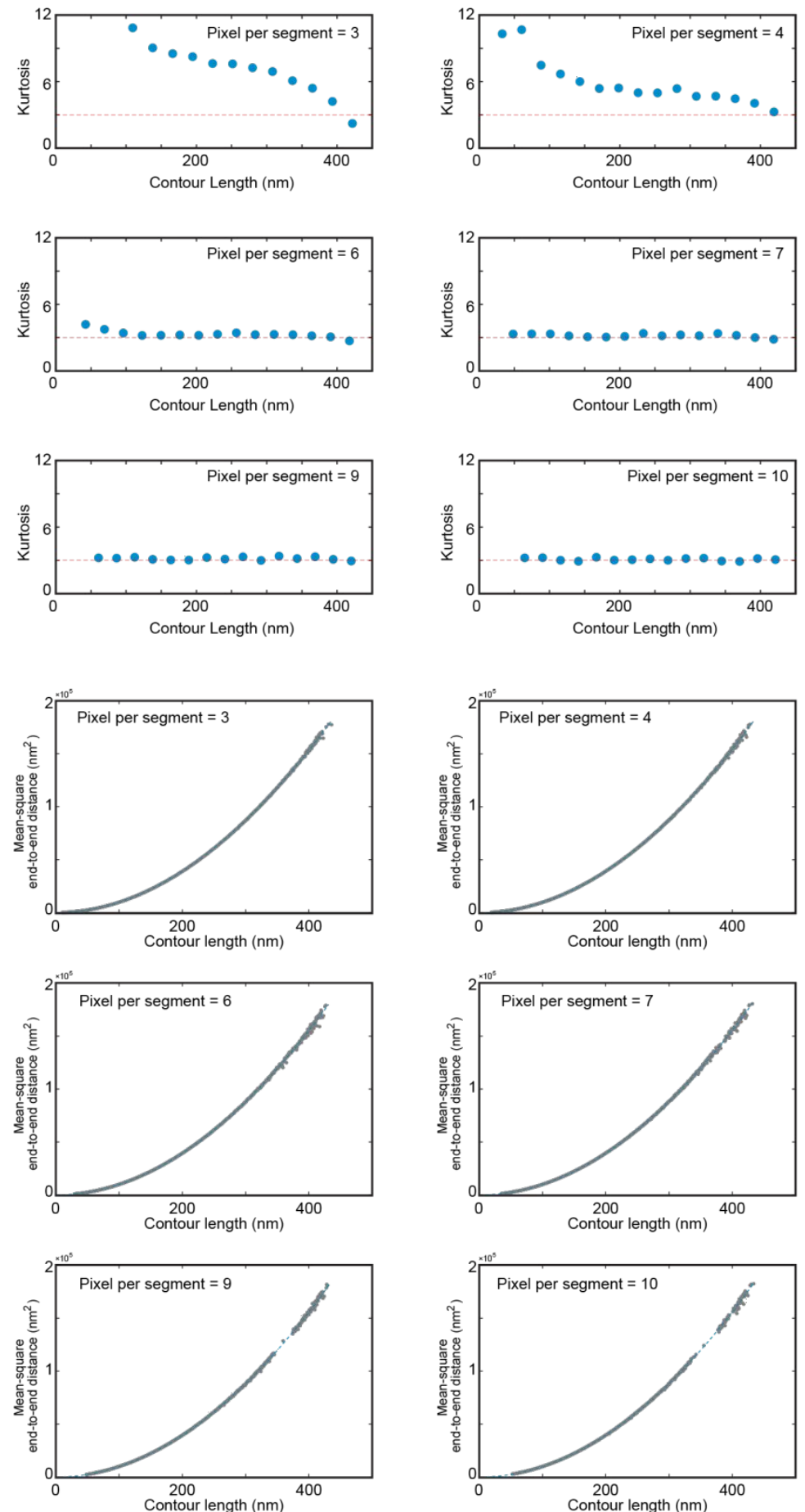

Supplementary Fig. 39. Parameter analysis on 6HB-Ref design. (a) Kurtosis analysis and (b) endto-end distance fitting curves. Calculated bending persistence length in each case was shown in Supplementary Fig. 37b. Pixel per segment value was chosen as 5 in the manuscript. 

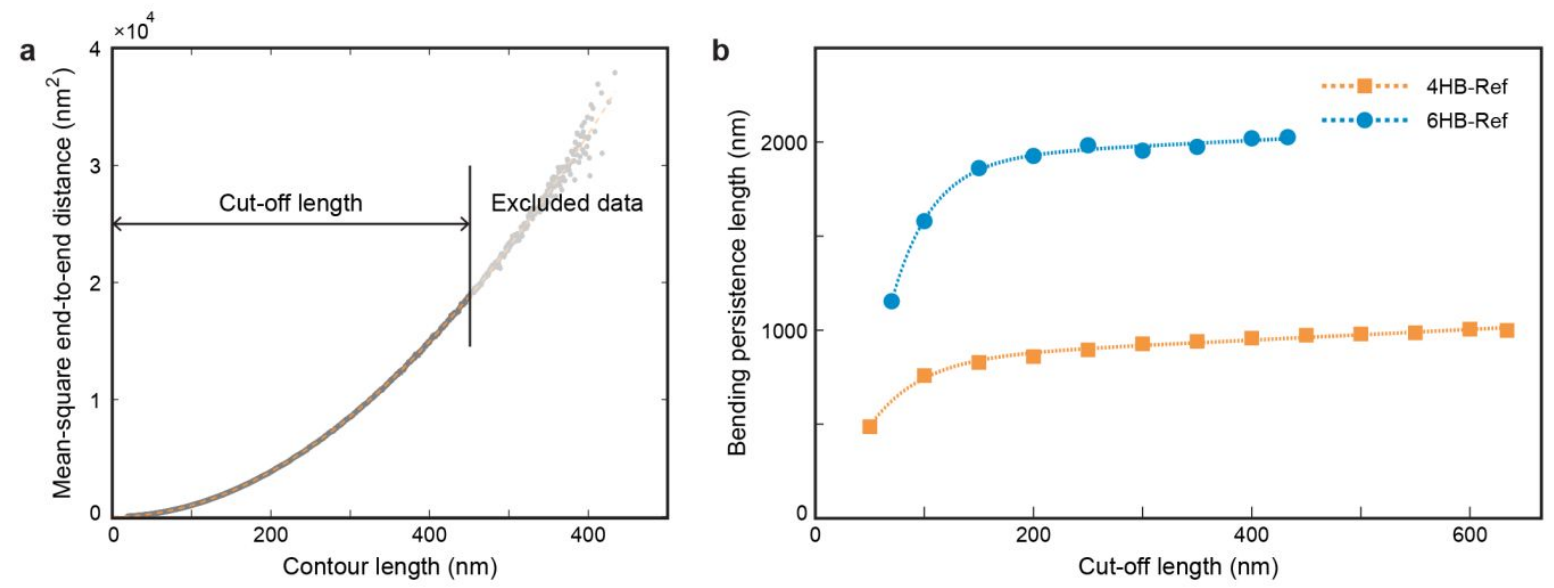

Supplementary Fig. 40. Calculation of bending persistence length while varying the range of contour length. (a) Definition of the cut-off length. Data within cut-off contour length was used to calculate the bending persistence length. (b) A graph showing that calculated values of bending persistence length were converged within monomer length range both in 4HB-Ref and 6HB-Ref design. 


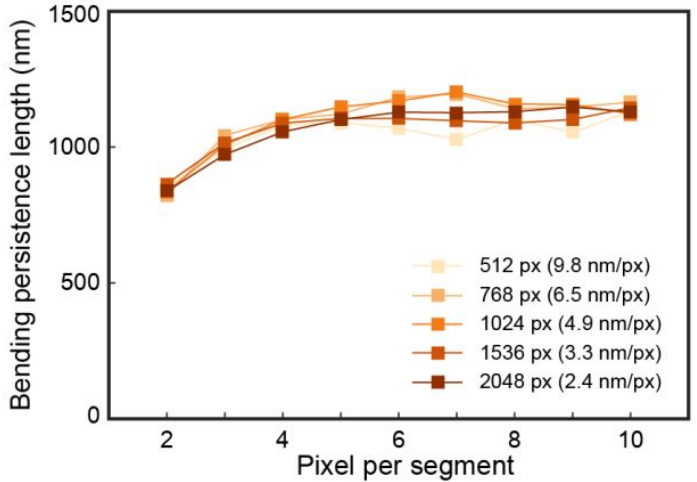

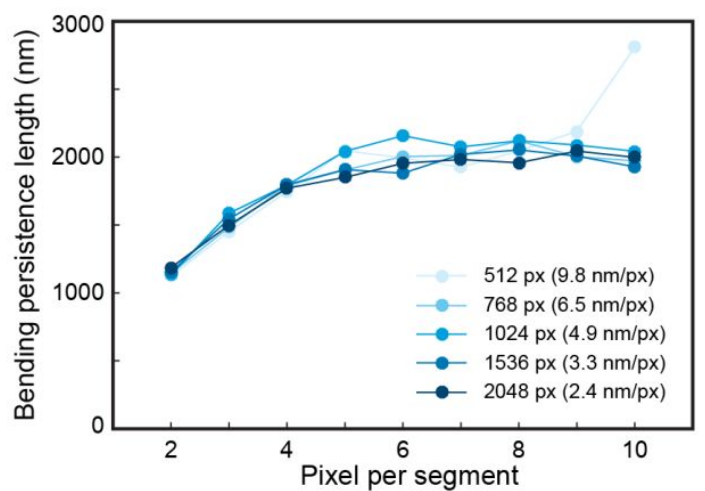

Supplementary Fig. 41. Calculation of bending persistence length while varying the resolution of the images. (a) Results of 4HB-Ref design using 100 monomers and (b) 6HB-Ref design using 140 monomers. From the analysis, 1024 px resolution was used in all of the cases shown in the manuscript. 


\section{Anisotropic gap distribution of $6 \mathrm{HB}$ design in longitudinal direction}

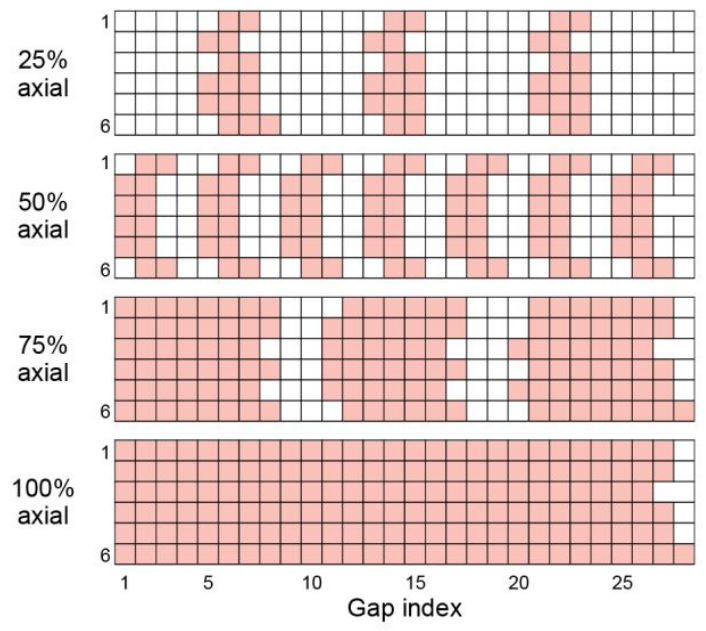

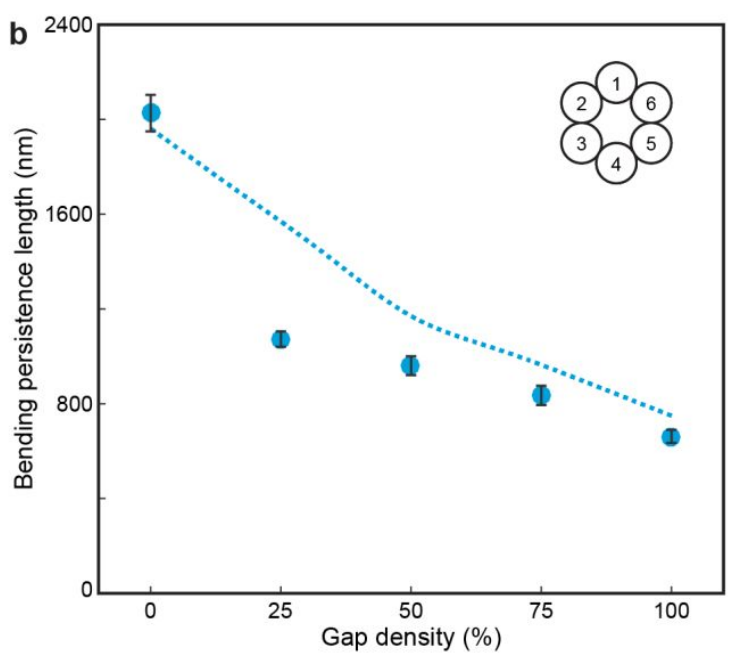

Supplementary Fig. 42. Anisotropic gap distribution result. (a) Schematic illustration of anisotropic gap distributions in a longitudinal direction. (b) Experimentally measured values of bending persistence length. Blue dotted line indicates the spline-fitted FE simulation result. Error bars indicate the standard deviation of experimental results. 
a

6HB-5nt-25\%-Axial

Number of samples $=233$

Mean bending persistence length $=1190 \mathrm{~nm}$

Stdev. bending persistence length $=40 \mathrm{~nm}$

$R^{2}=0.9984$
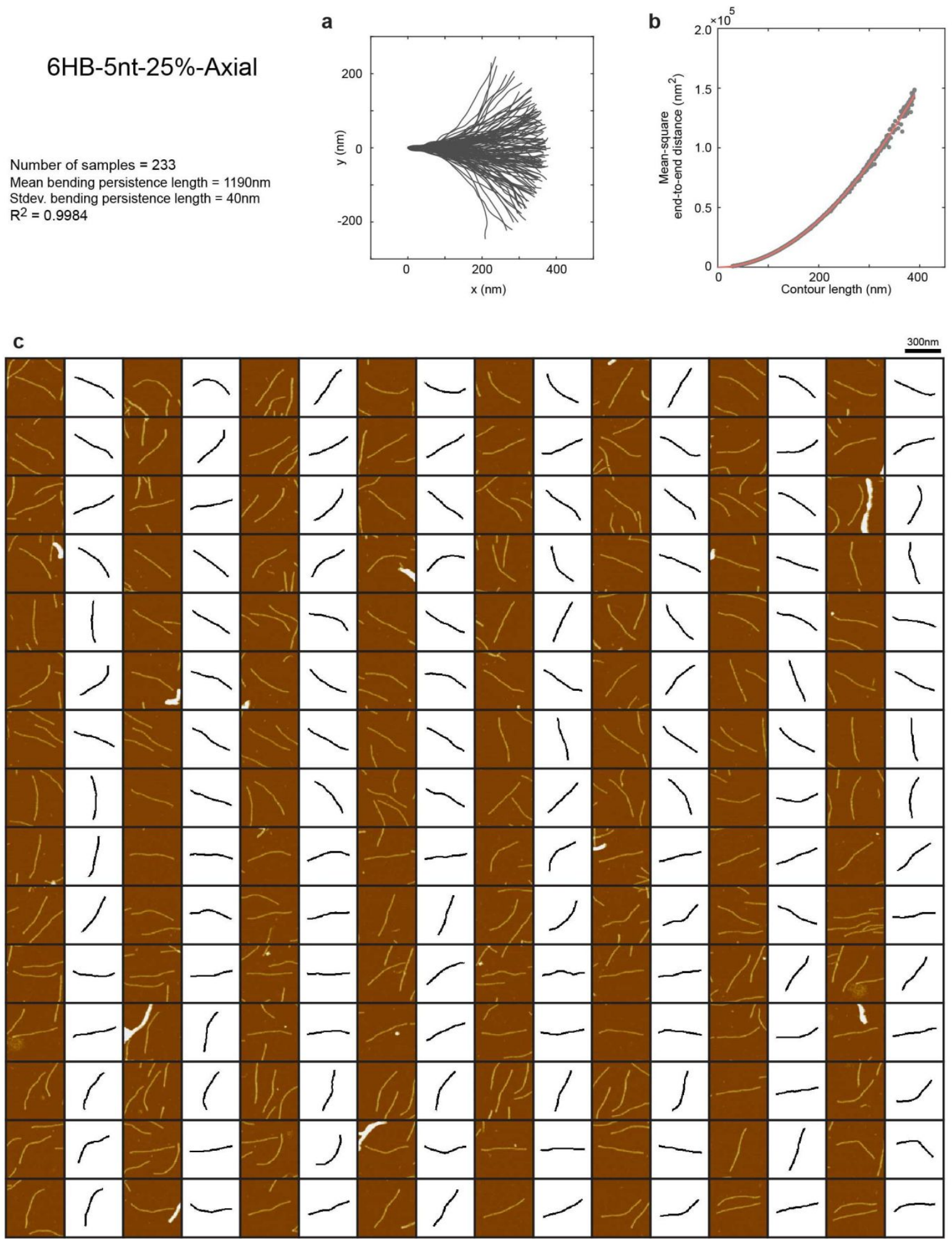

Supplementary Fig. 43. Experimental characterization of 6HB-5nt-25\%-Axial design. (a) Aligned contour distribution of 120 representative monomers. (b) Calculation of the mean bending persistence length by fitting all measured data. (c) AFM images and extracted monomer contours shown in (a). 
a

6HB-5nt-50\%-Axial

Number of samples $=\mathbf{5 7 4}$

Mean bending persistence length $=1020 \mathrm{~nm}$

Stdev. bending persistence length $=50 \mathrm{~nm}$

$R^{2}=0.9980$
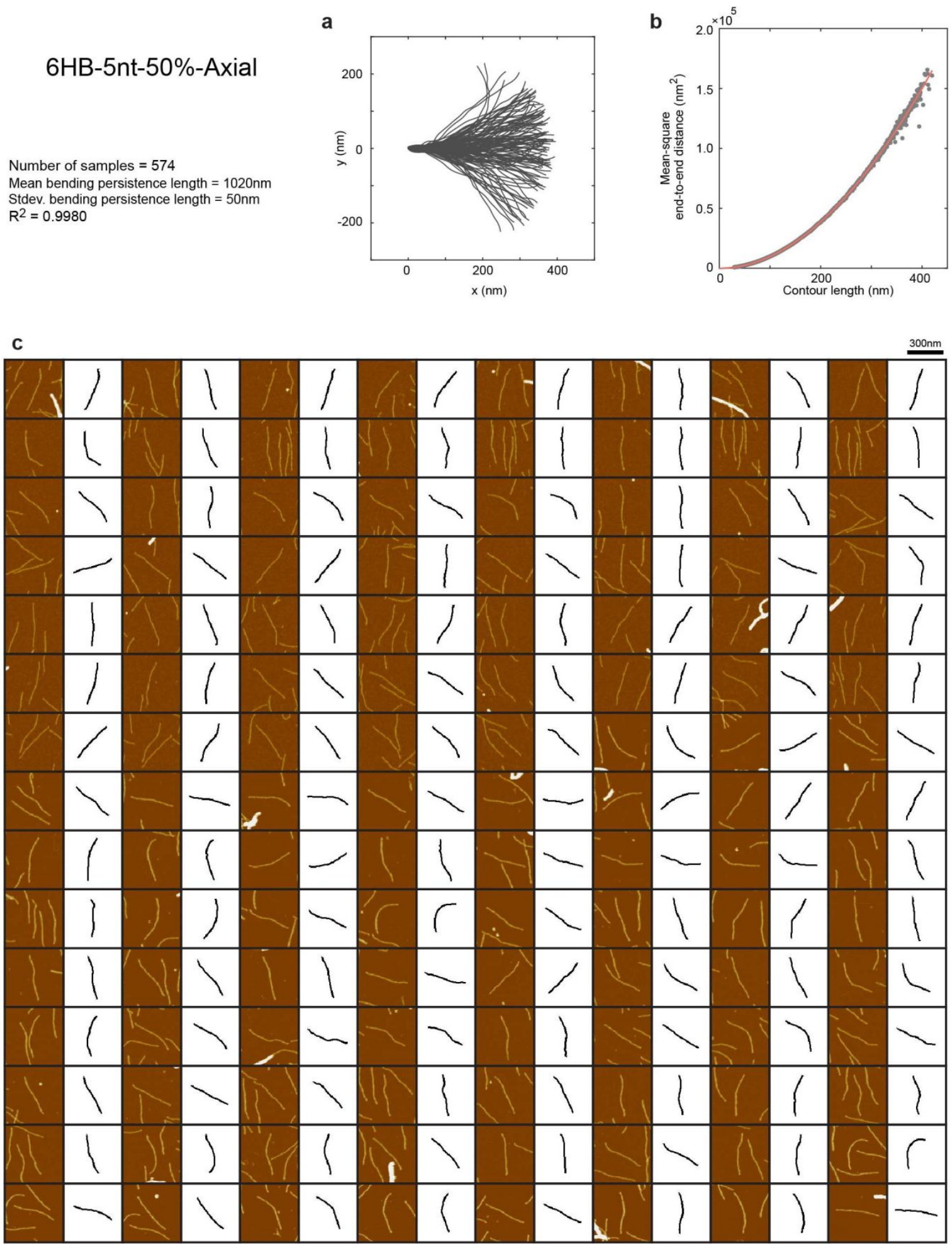

Supplementary Fig. 44. Experimental characterization of 6HB-5nt-50\%-Axial design. (a) Aligned contour distribution of 120 representative monomers. (b) Calculation of the mean bending persistence length by fitting all measured data. (c) AFM images and extracted monomer contours shown in (a). 
a

6HB-5nt-75\%-Axial

Number of samples $=600$

Mean bending persistence length $=900 \mathrm{~nm}$

Stdev. bending persistence length $=40 \mathrm{~nm}$

$R^{2}=0.9980$
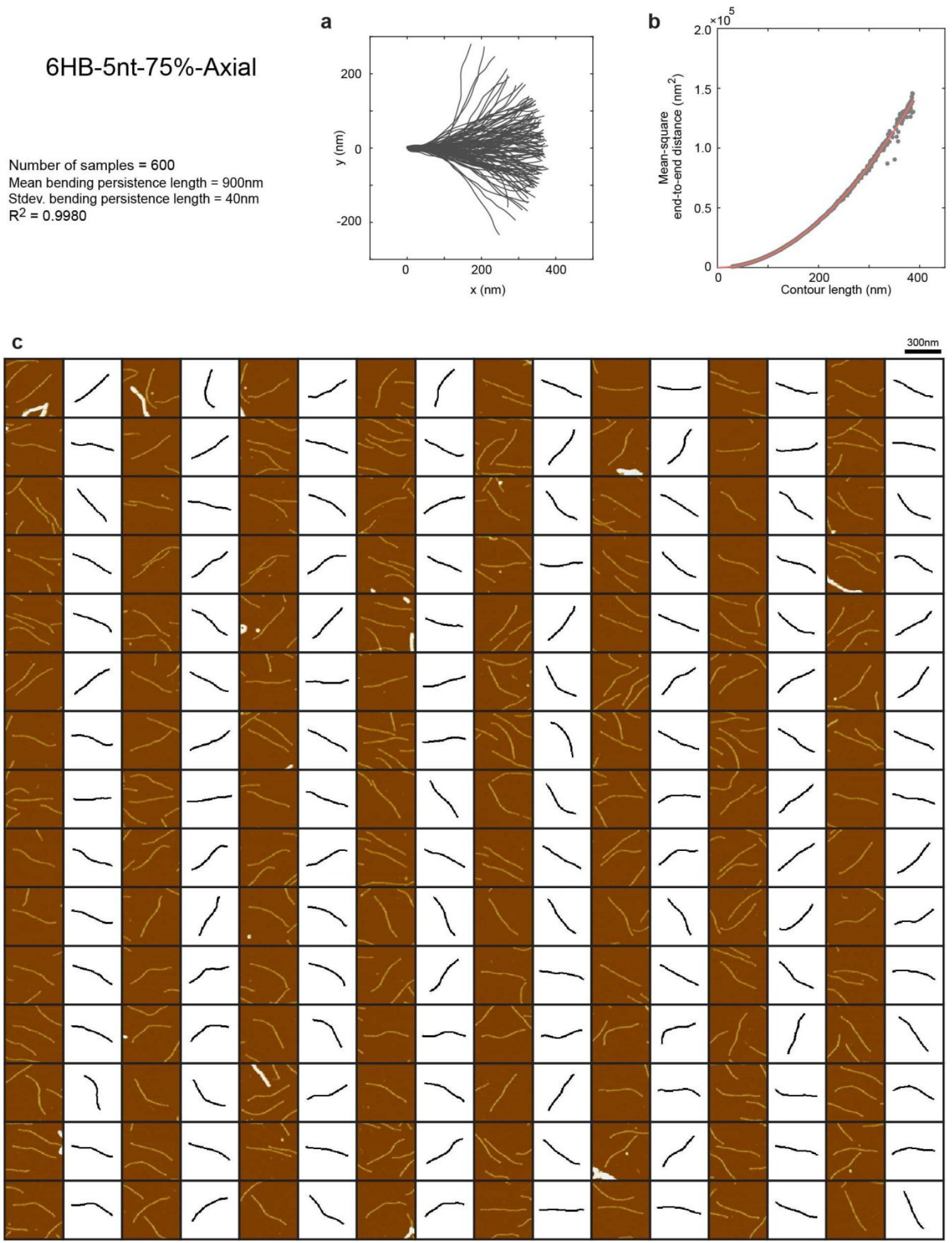

Supplementary Fig. 45. Experimental characterization of 6HB-5nt-75\%-Axial design. (a) Aligned contour distribution of 120 representative monomers. (b) Calculation of the mean bending persistence length by fitting all measured data. (c) AFM images and extracted monomer contours shown in (a). 


\section{Folding yield analysis}
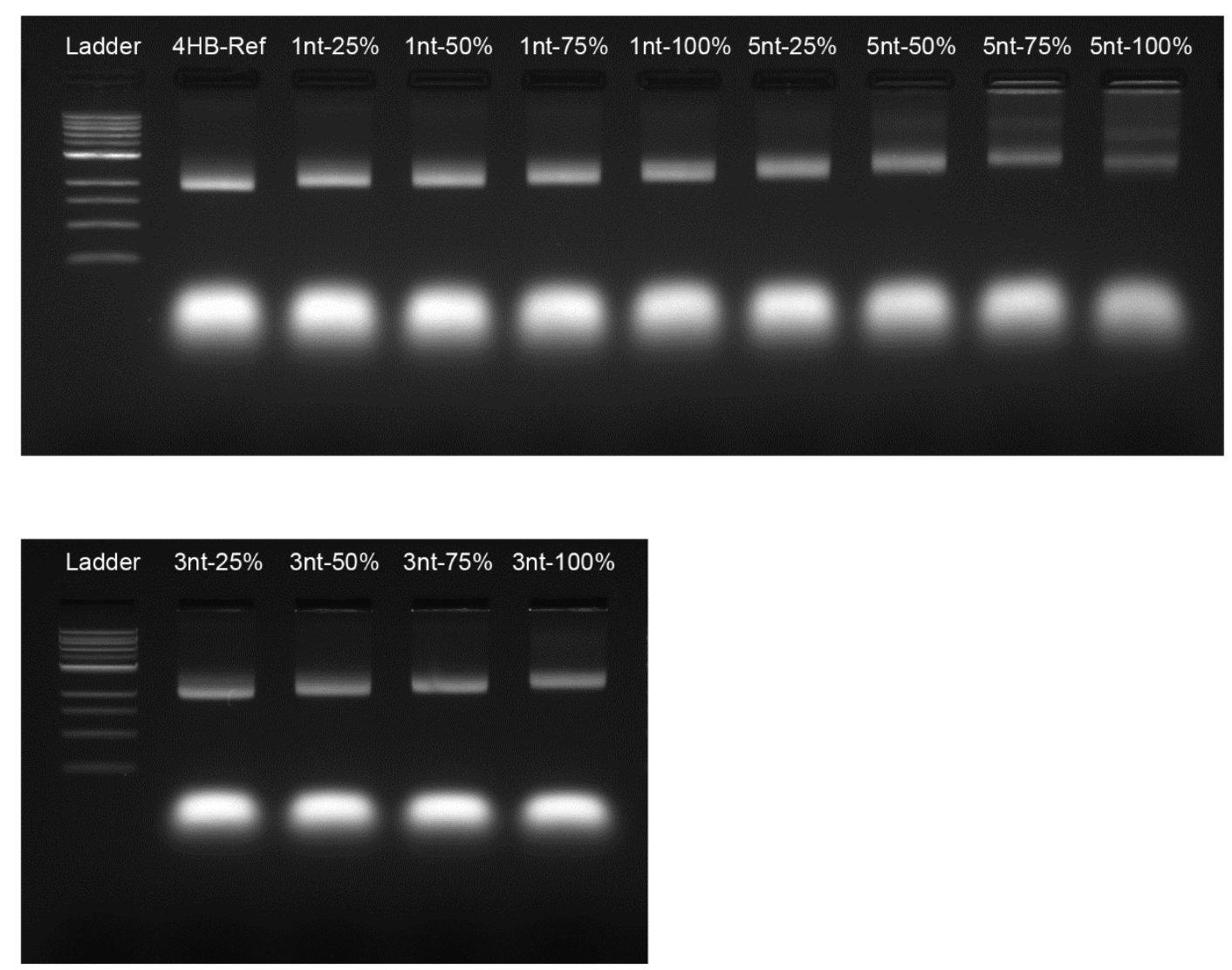

Supplementary Fig. 46. Gel electrophoresis result of 4HB designs. 

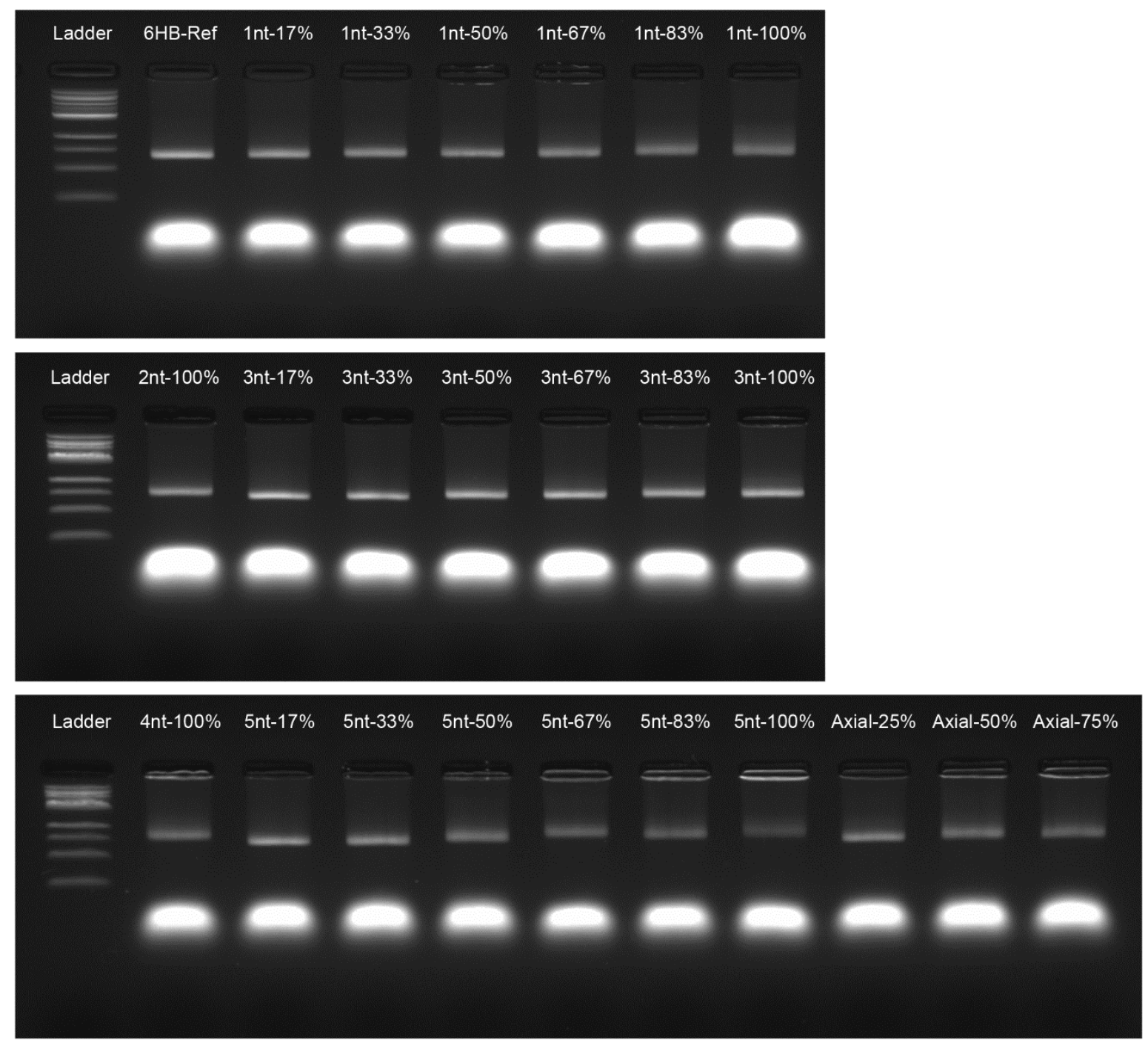

Supplementary Fig. 47. Gel electrophoresis result of $6 \mathrm{HB}$ designs. 


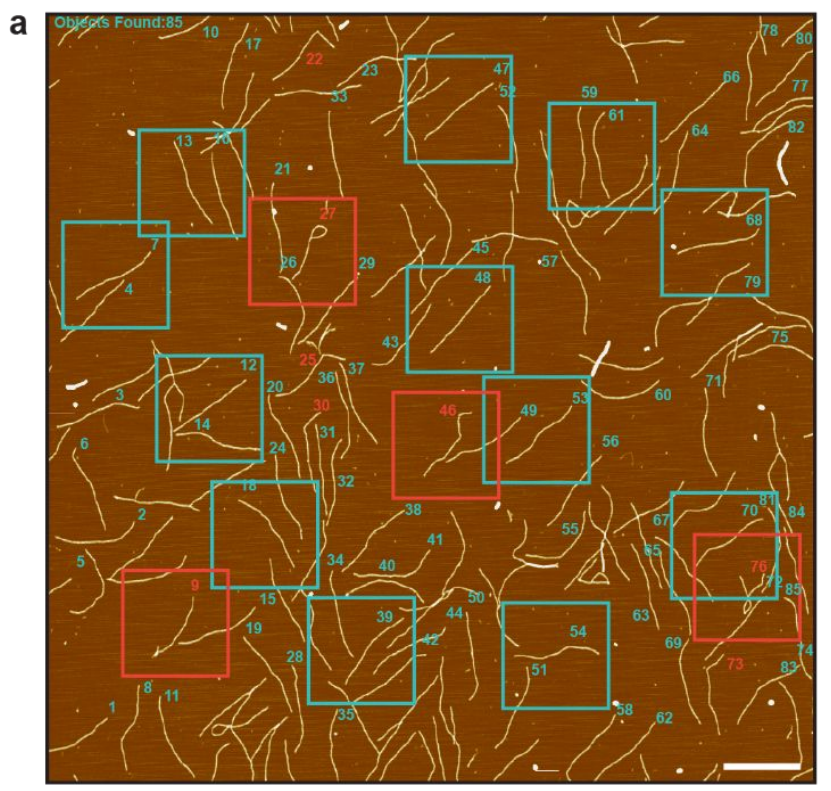

b Well-folded structures

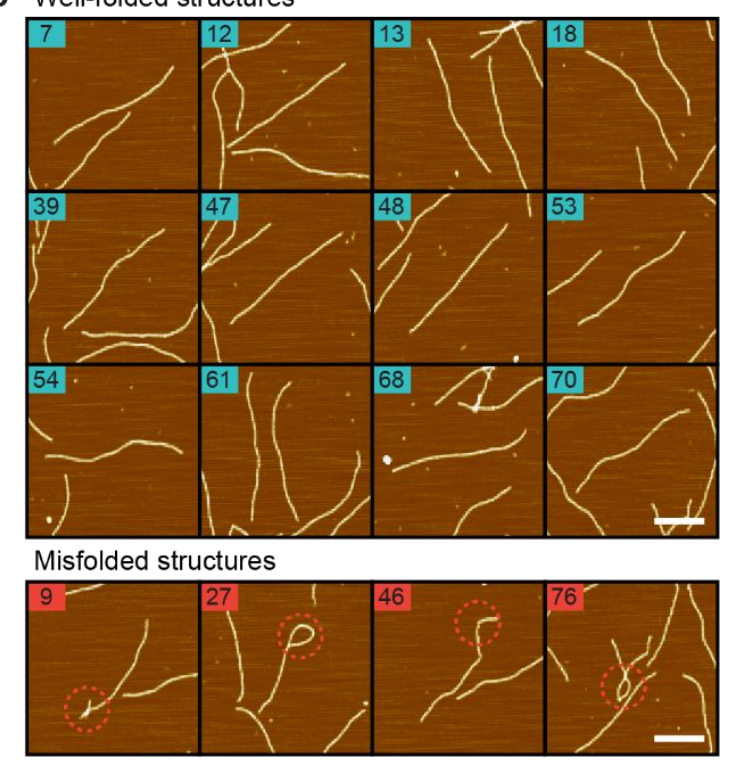

Supplementary Fig. 48. Structural folding yield analysis. (a) AFM image of the 4HB-Ref design showing the process of structural folding yield calculation. After the automated monomer-selection process, well-folded and misfolded monomer structures were classified manually. Misfolded monomers having any kind of a sharp kink, rolled shape and visible collapsed region, were excluded in the measurement of persistence length. Result of all design variations were summarized in Supplementary Table 2. Scale bar: $500 \mathrm{~nm}$ (b) Representative monomer images of well-folded and misfolded monomer structures. Red dotted circles indicate the location of misfolded regions. Scale bars: $200 \mathrm{~nm}$. 


\section{Alternative approaches for the stiffness control}

a

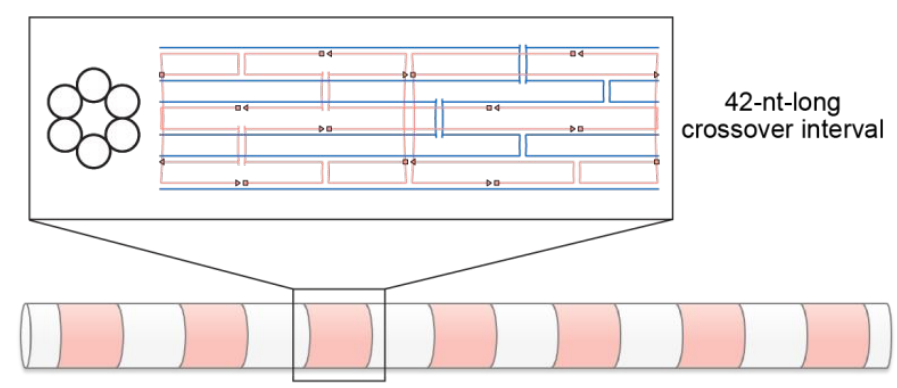

b
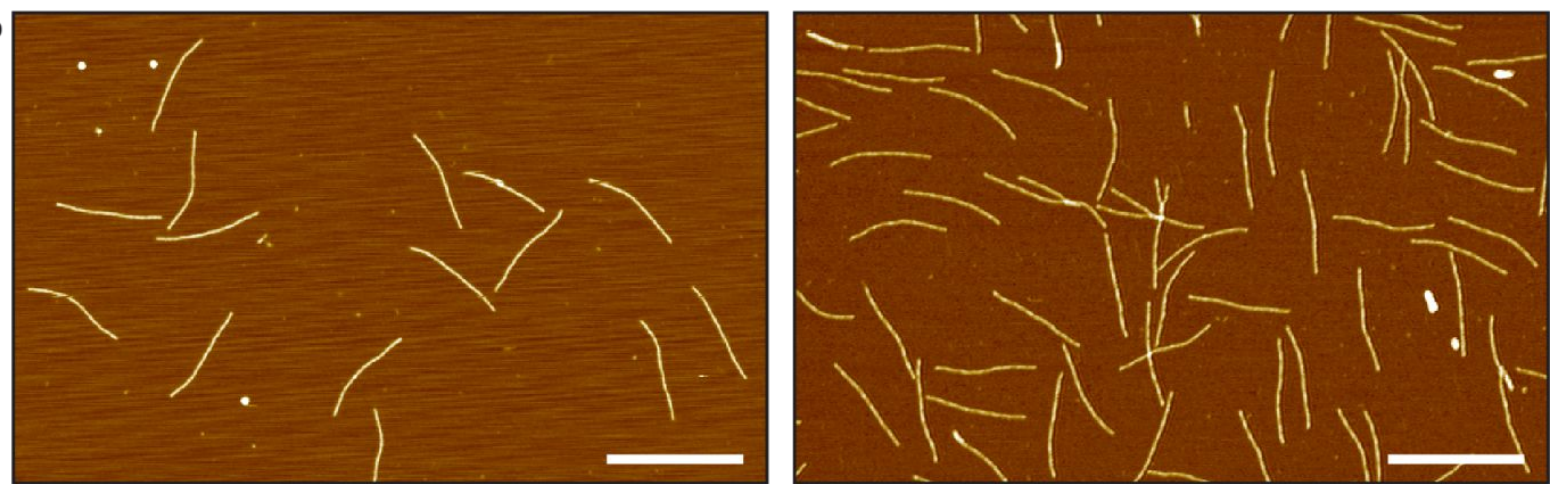

Supplementary Fig. 49. 6HB bundle with 42-nt-interval crossover modification at half of the region. (a) Schematic illustration showing the modified regions and staple design therein. (b) Representative AFM images of the reference and 42-nt-long crossover design, respectively. Scale bars: $300 \mathrm{~nm}$. 
a

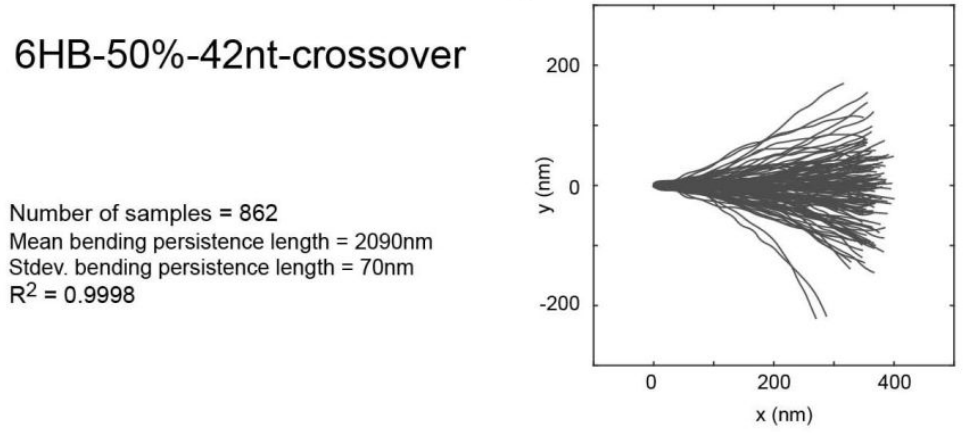

b

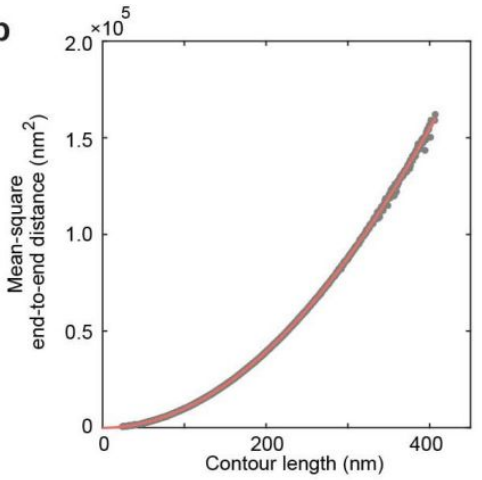

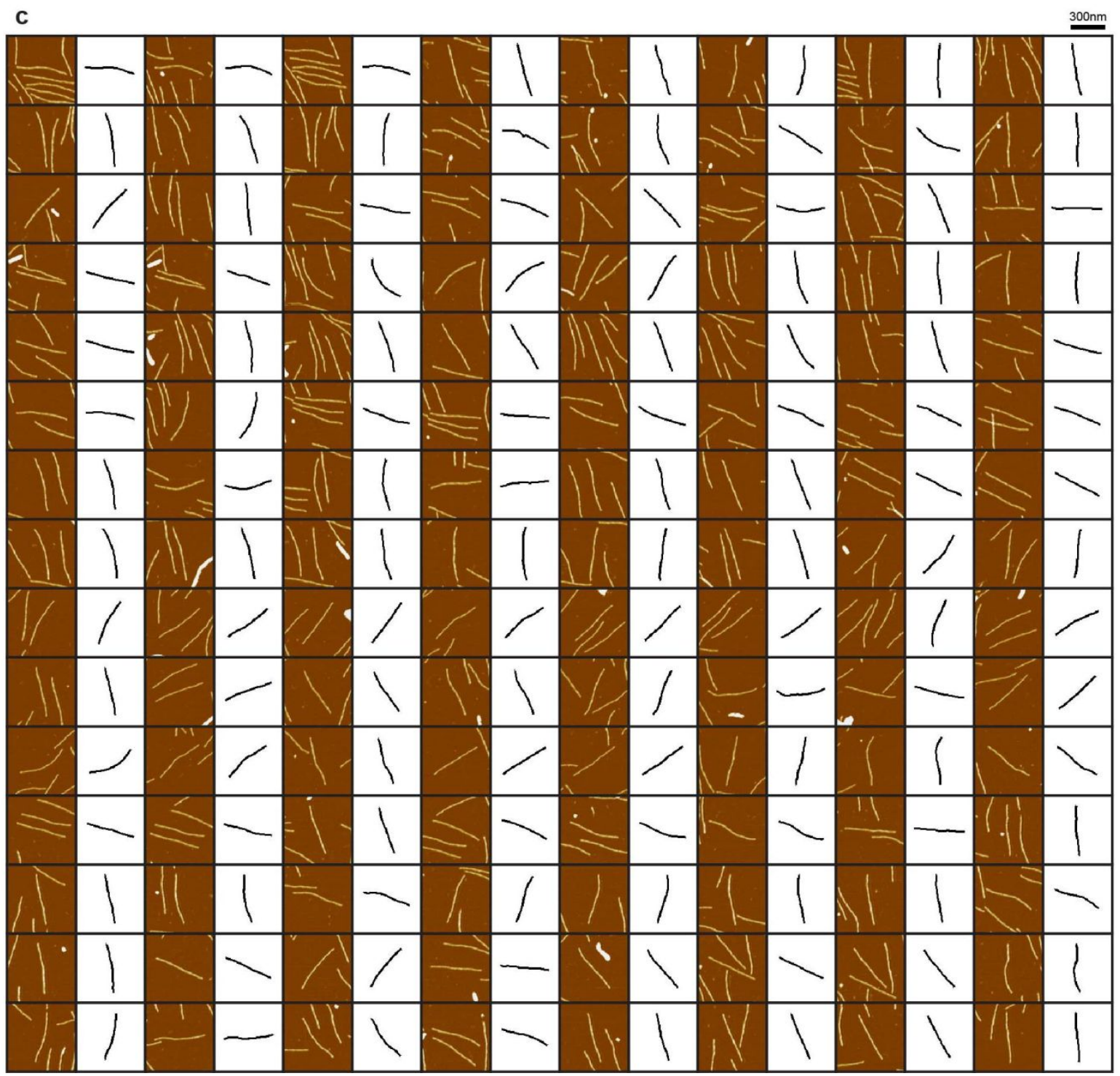

Supplementary Fig. 50. Experimental characterization of 6HB-50\%-42nt-crossover design. (a) Aligned contour distribution of 120 representative monomers. (b) Calculation of the mean bending persistence length by fitting all measured data. (c) AFM images and extracted monomer contours shown in (a). 
a

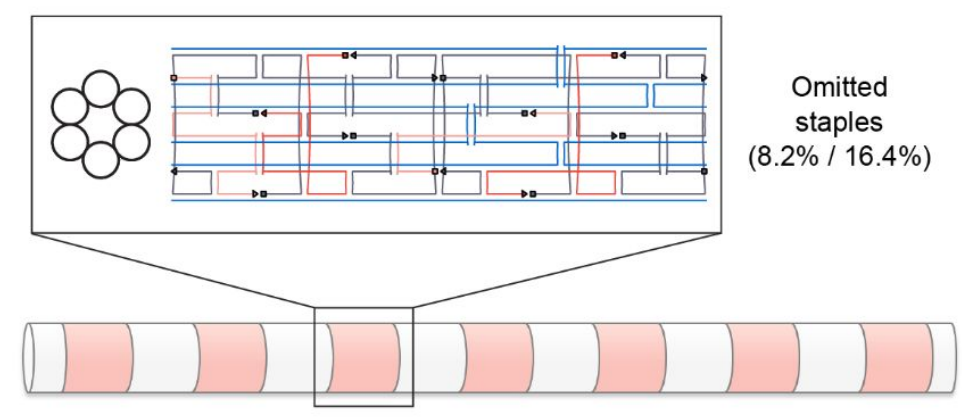

b
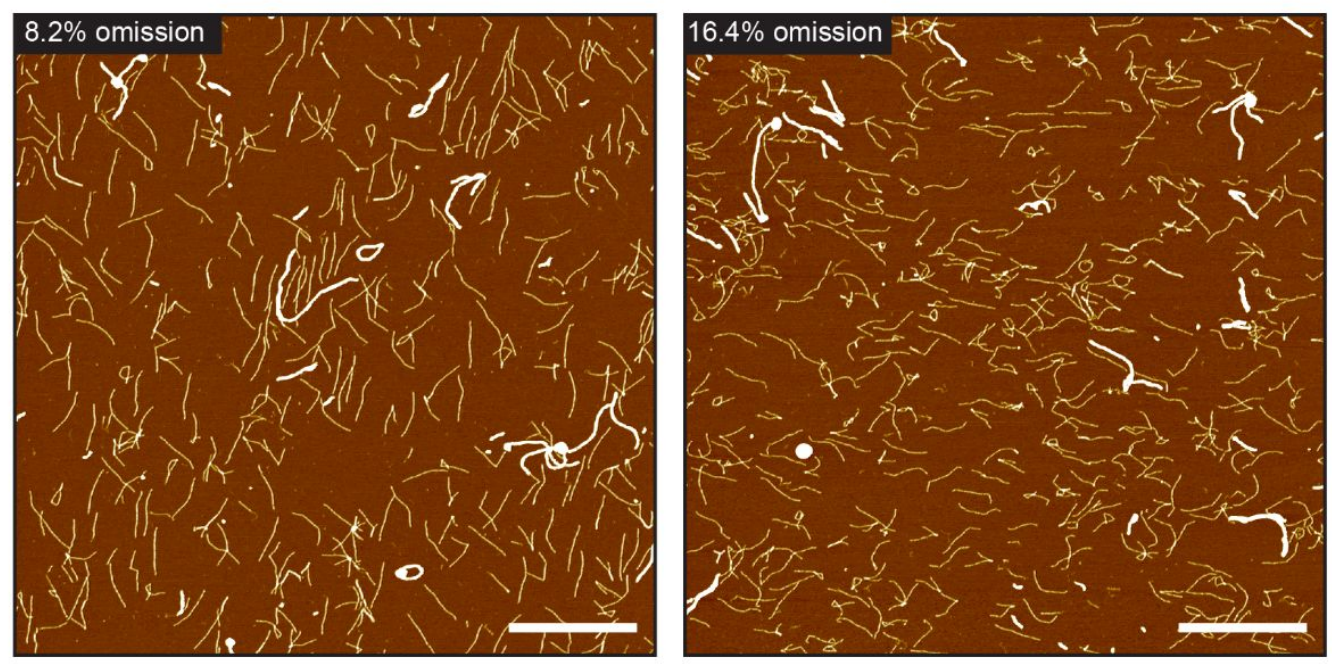

Supplementary Fig. 51. 6HB bundle with staple omission at half of the region. (a) Schematic illustration showing the modified regions and representative positions of omitted staples therein. Note that positions of omitted staples were changed along the regions to distribute the defect locations. Two light red-colored staples were removed in 8.2\% omission design, and another two darker red staples were additionally removed in $16.4 \%$ omission design. (b) AFM image of the two cases. Scale bars: $1 \mu \mathrm{m}$. 


\section{Finite element modeling of defect-engineered DNA nanostructures}
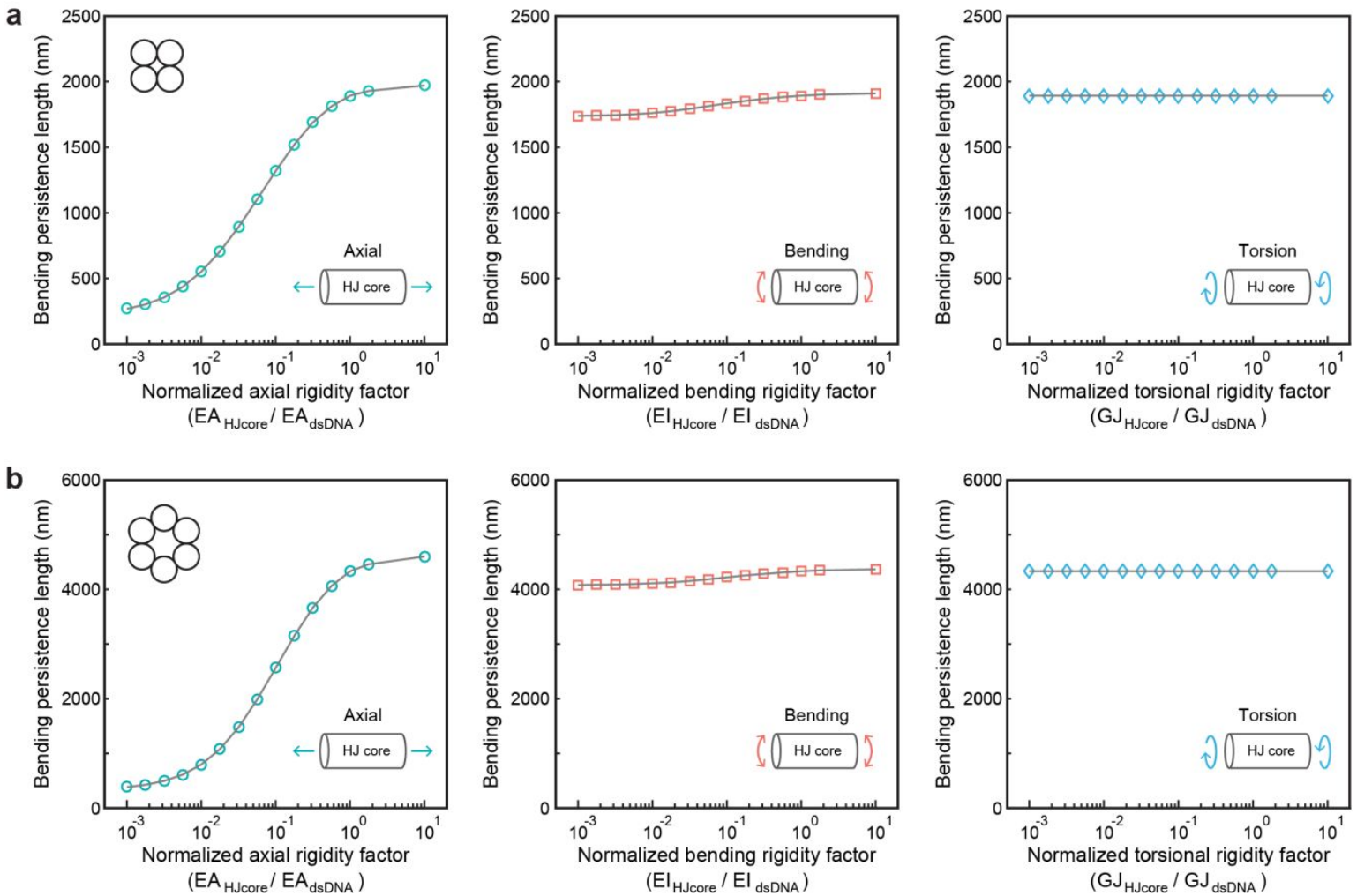

Supplementary Fig. 52. Sensitivity analysis for the HJcore element. Calculated bending persistence length of the bundle in (a) 4HB-Ref and (b) 6HB-Ref design was shown. While varying axial, bending, or torsional stiffness of the HJcore element, the other two normalized parameters were fixed as 1 . 

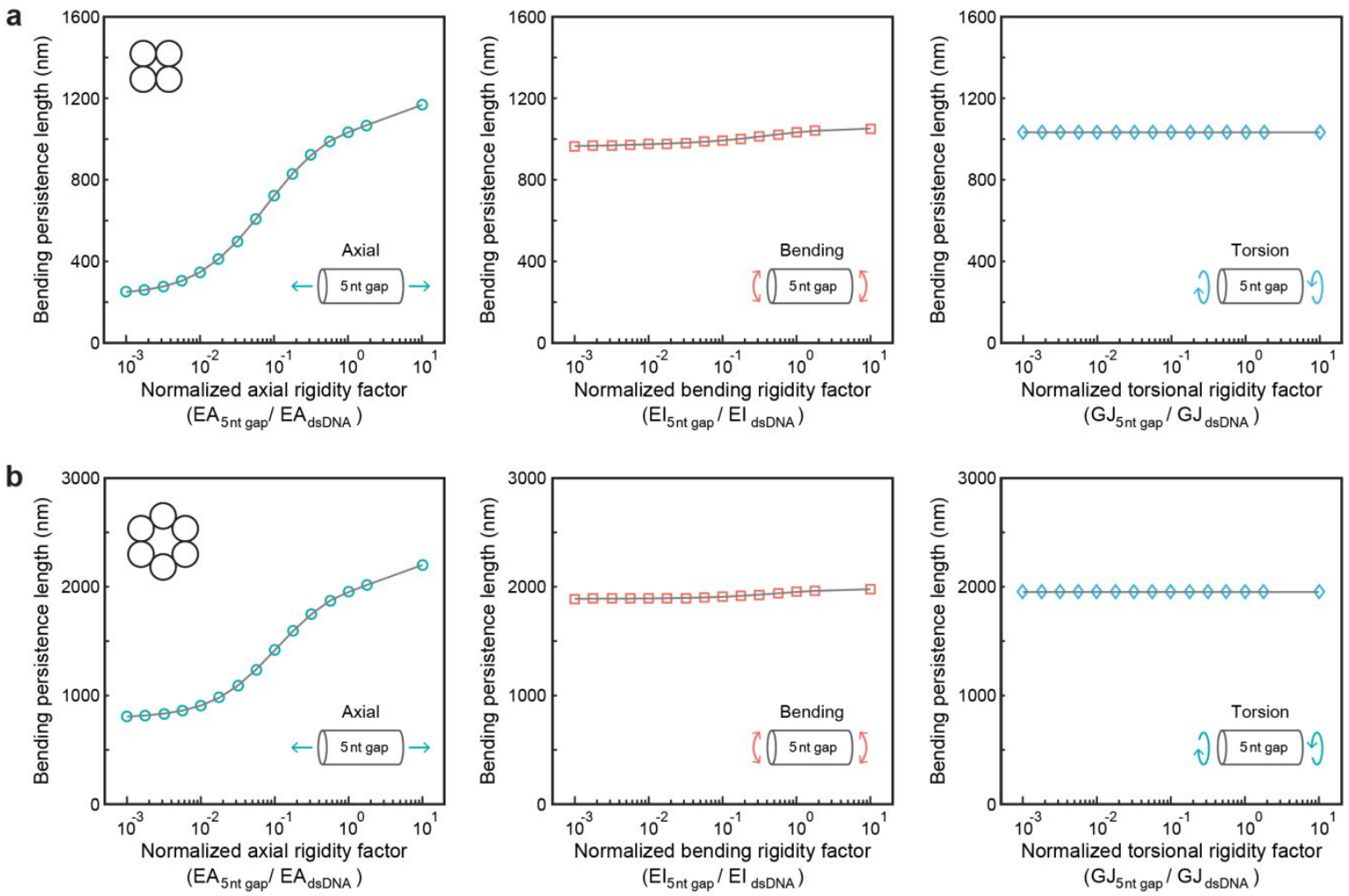

Supplementary Fig. 53. Sensitivity analysis for the 5-nt-long ssDNA gap element. Calculated bending persistence length of the bundle in (a) 4HB-5nt-100\% and (b) 6HB-5nt-100\% design was shown. Stiffness factor of the HJcore element used here was shown in Supplementary Table 3. While varying axial, bending, or torsional stiffness of the 5-nt-long ssDNA gap element, the other two normalized parameters were fixed as 1 . 


\section{Molecular dynamics simulation}

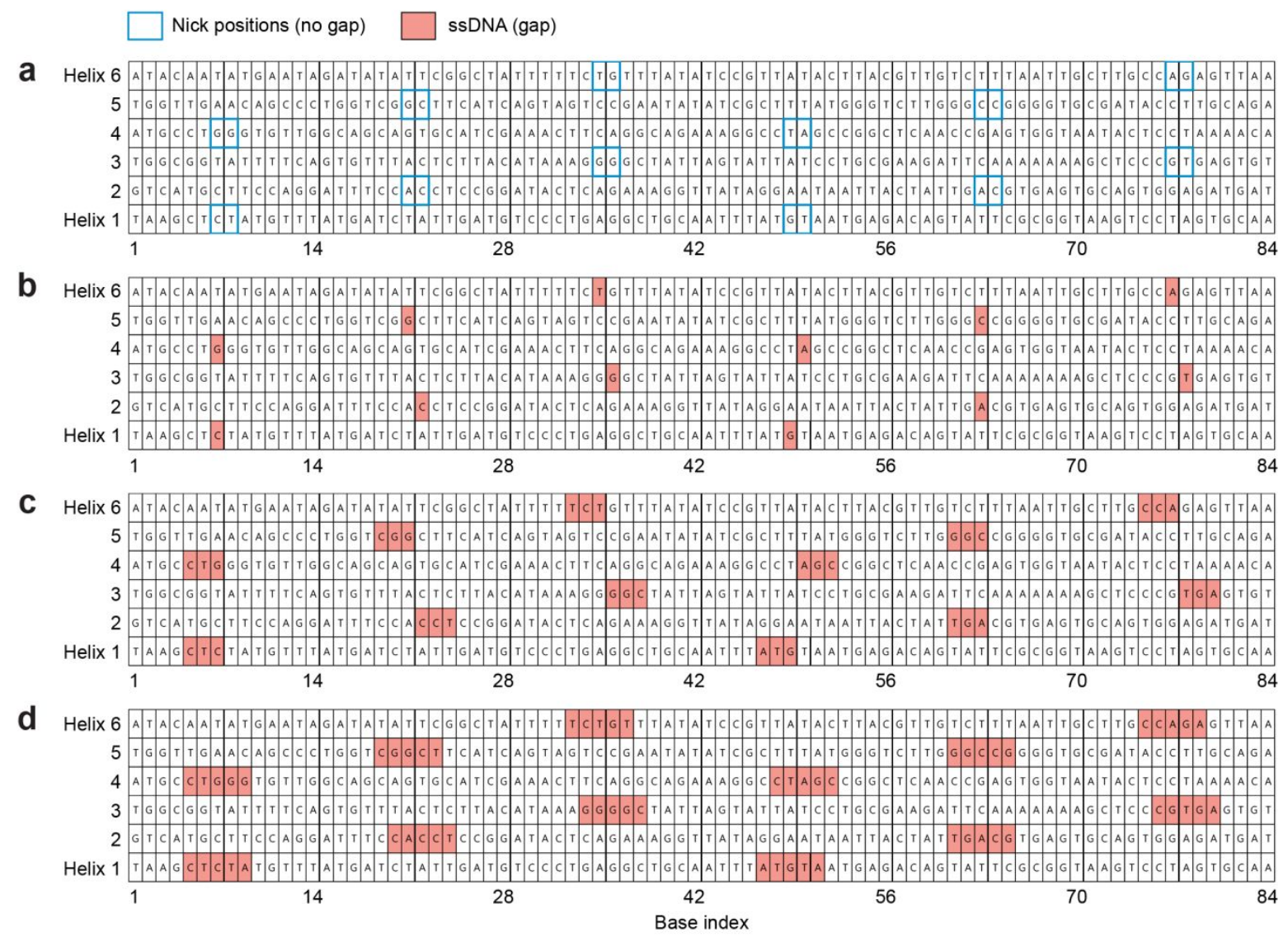

Supplementary Fig. 54. DNA sequence used in MD simulation. The sequence of scaffold strand was shown in (a) no gap, (b) 1-nt-long gap, (c) 3-nt-long gap, and (d) 5-nt-long gap design. 
a
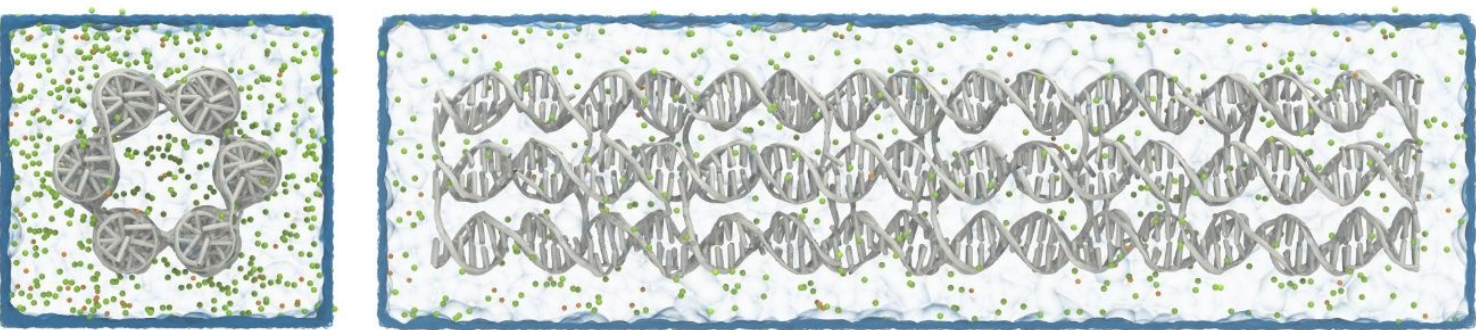

b
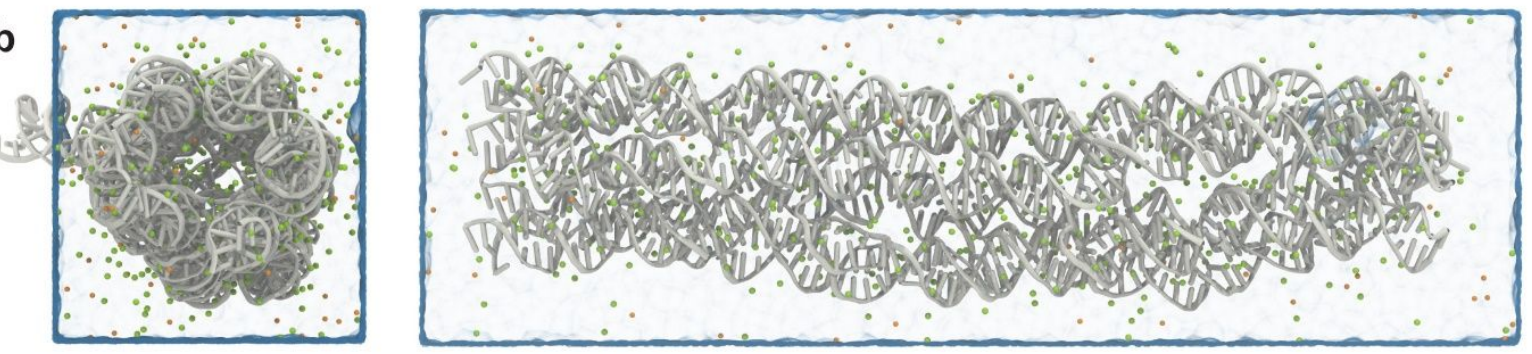

Supplementary Fig. 55. MD simulation of 6HB no gap structure. (a) Initial and (b) final (320 ns of simulation time) configuration of $6 \mathrm{HB}$ no gap bundle design. 
a
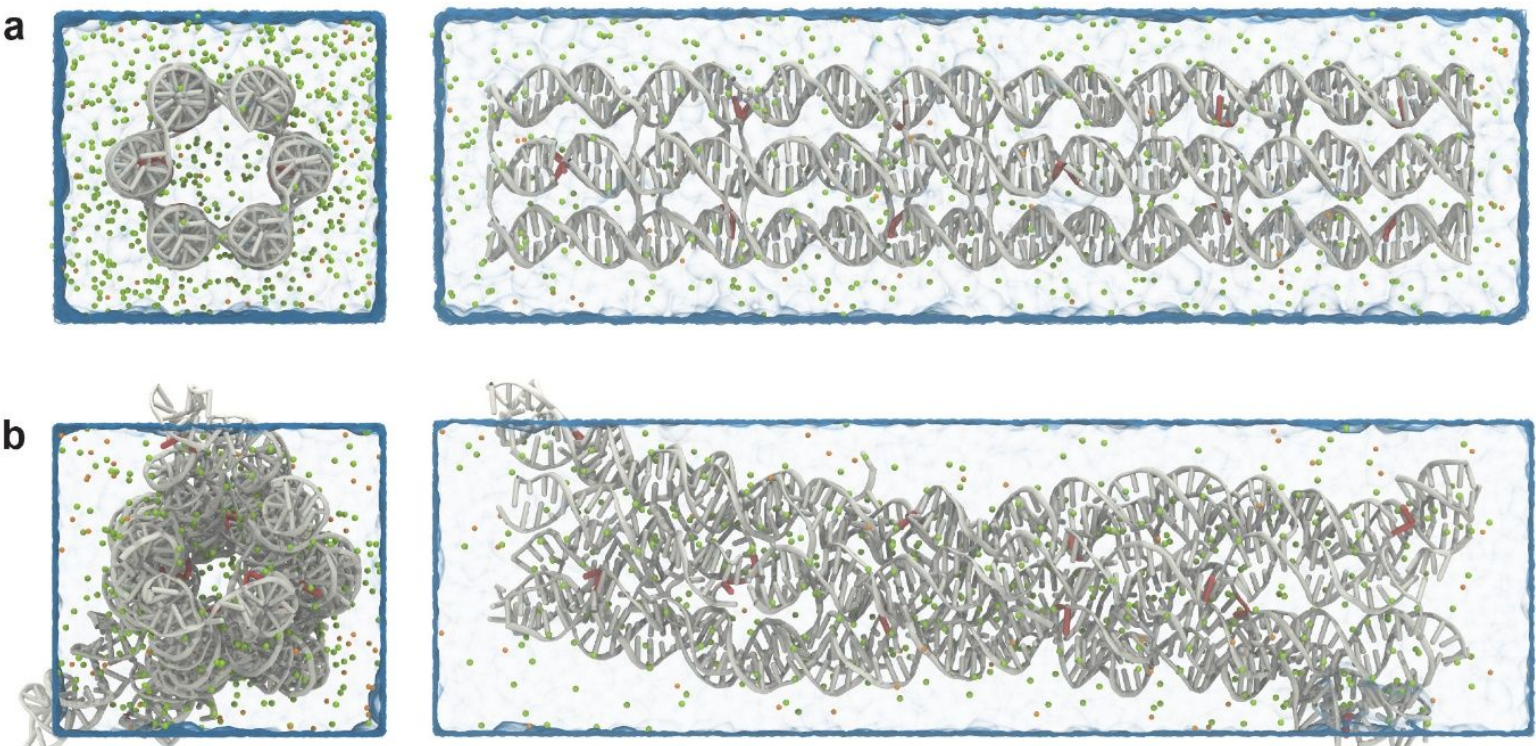

Supplementary Fig. 56. MD simulation of 6HB-1nt gap structure. (a) Initial and (b) final (320 ns of simulation time) configuration of 6HB-1nt gap bundle design. Red-colored bases indicate the gap positions. 
a

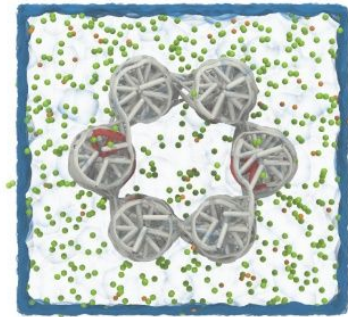

b

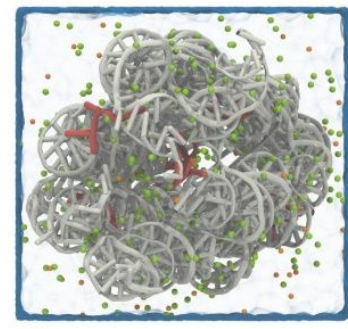

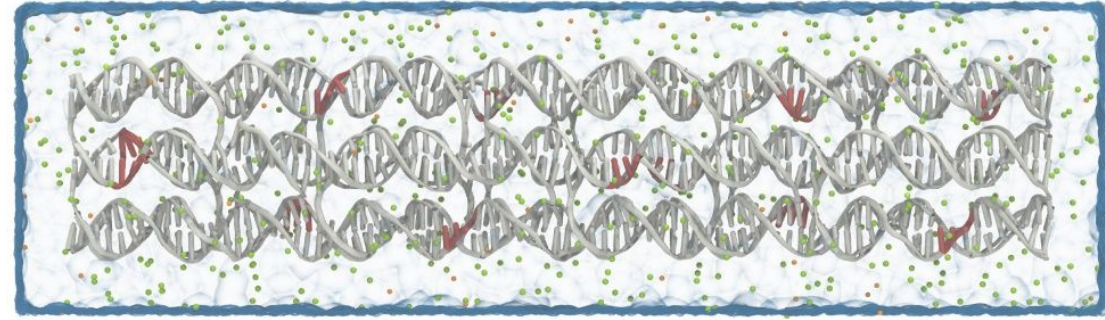

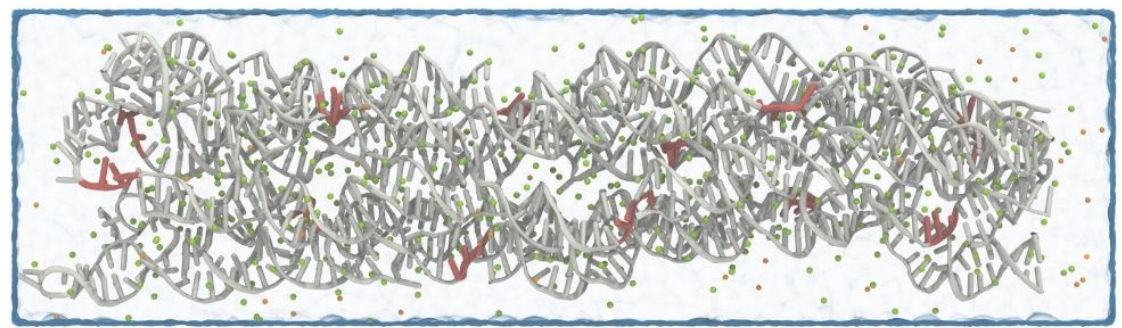

Supplementary Fig. 57. MD simulation of 6HB-3nt gap structure. (a) Initial and (b) final (320 ns of simulation time) configuration of 6HB-3nt gap bundle design. Red-colored bases indicate the gap positions. 

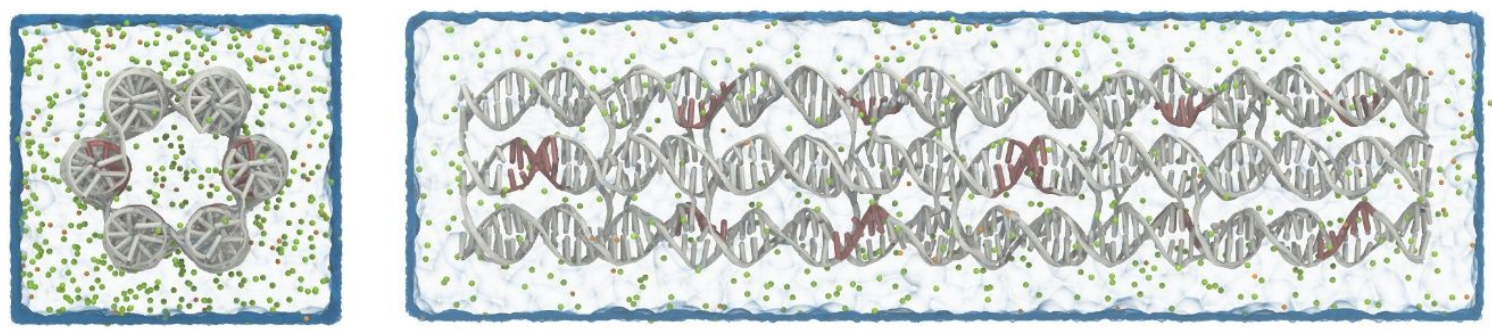

b
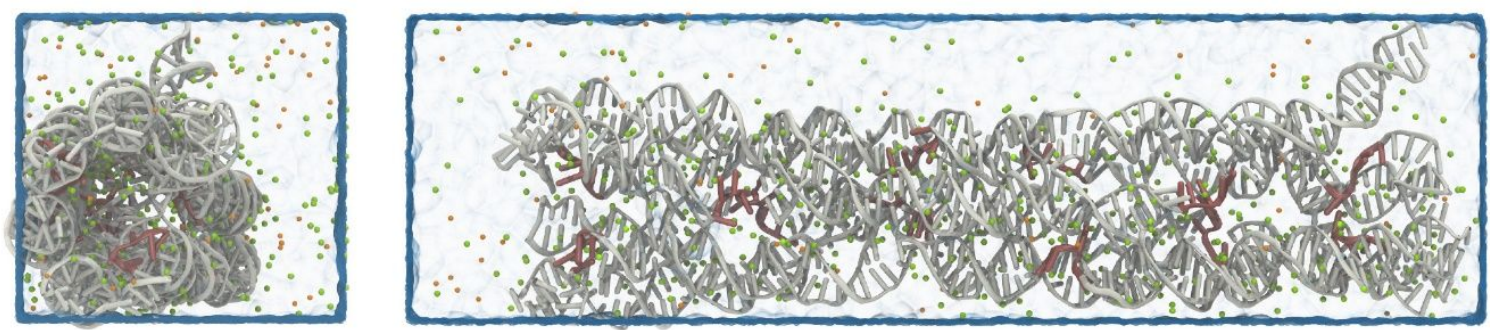

Supplementary Fig. 58. MD simulation of 6HB-5nt gap structure. (a) Initial and (b) final (320 ns of simulation time) configuration of 6HB-5nt gap bundle design. Red-colored bases indicate the gap positions. 


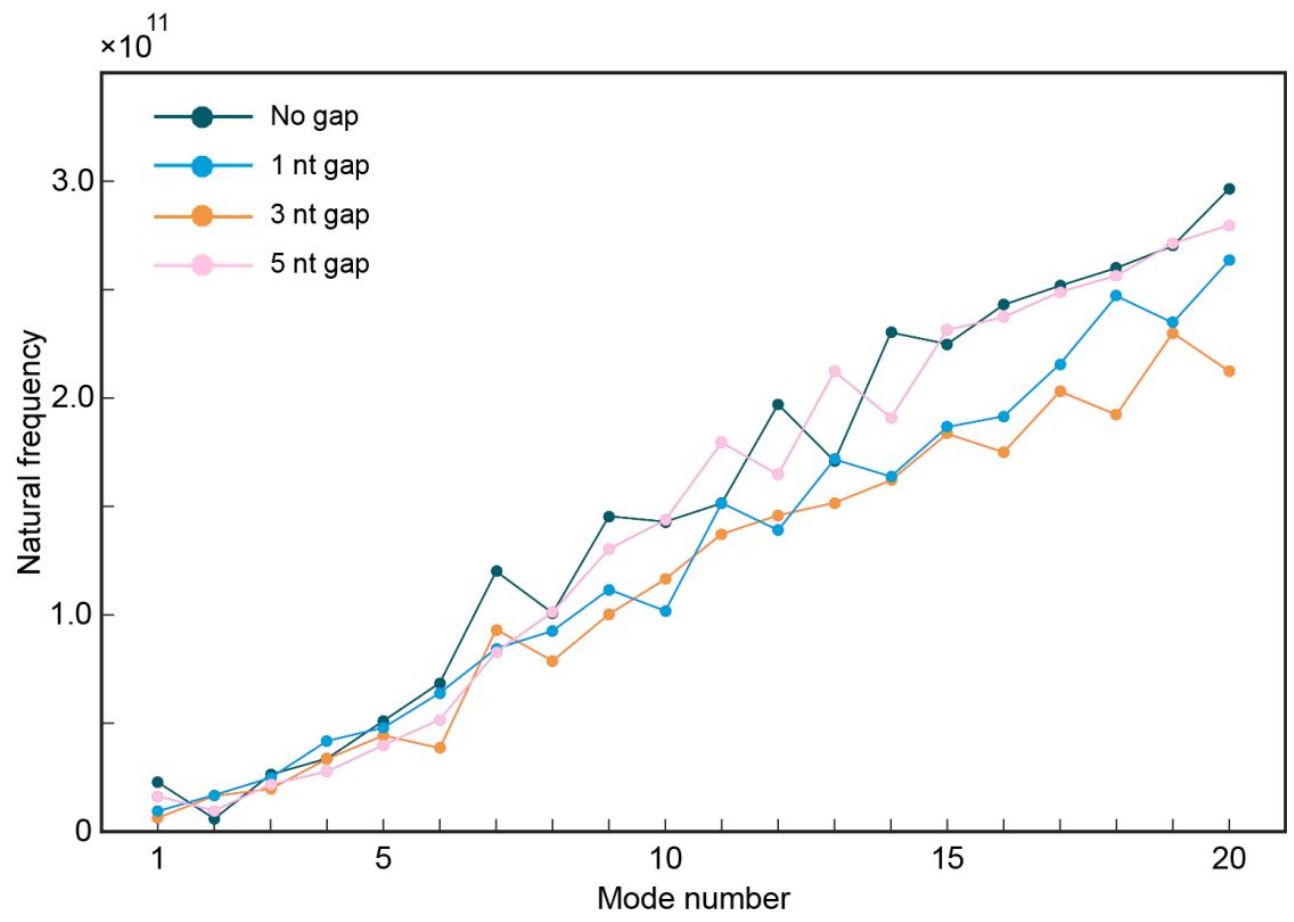

Supplementary Fig. 59. Principal component analysis of final 200-ns-long MD trajectories. Mode 7 was the first bending mode in no gap, 3-nt-long gap, and 5-nt-long gap designs, and mode 9 was the first bending mode in 1-nt-long gap design. 

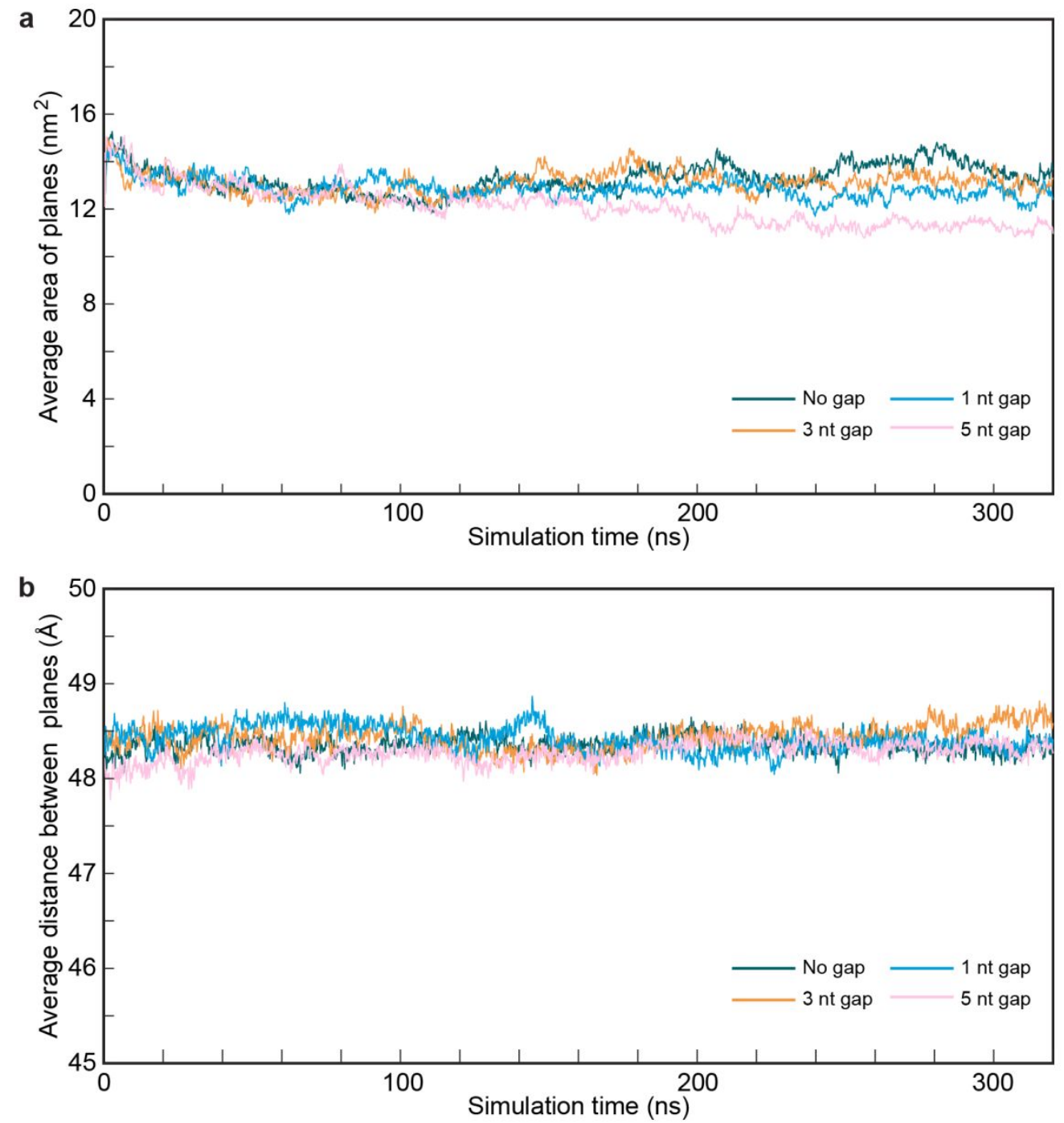

Supplementary Fig. 60. (a) Average area of planes and (b) average distance between planes during the MD simulation. Values of final 200-ns-long trajectories were further analyzed in Fig. 3e and $3 \mathrm{f}$ in the manuscript. 


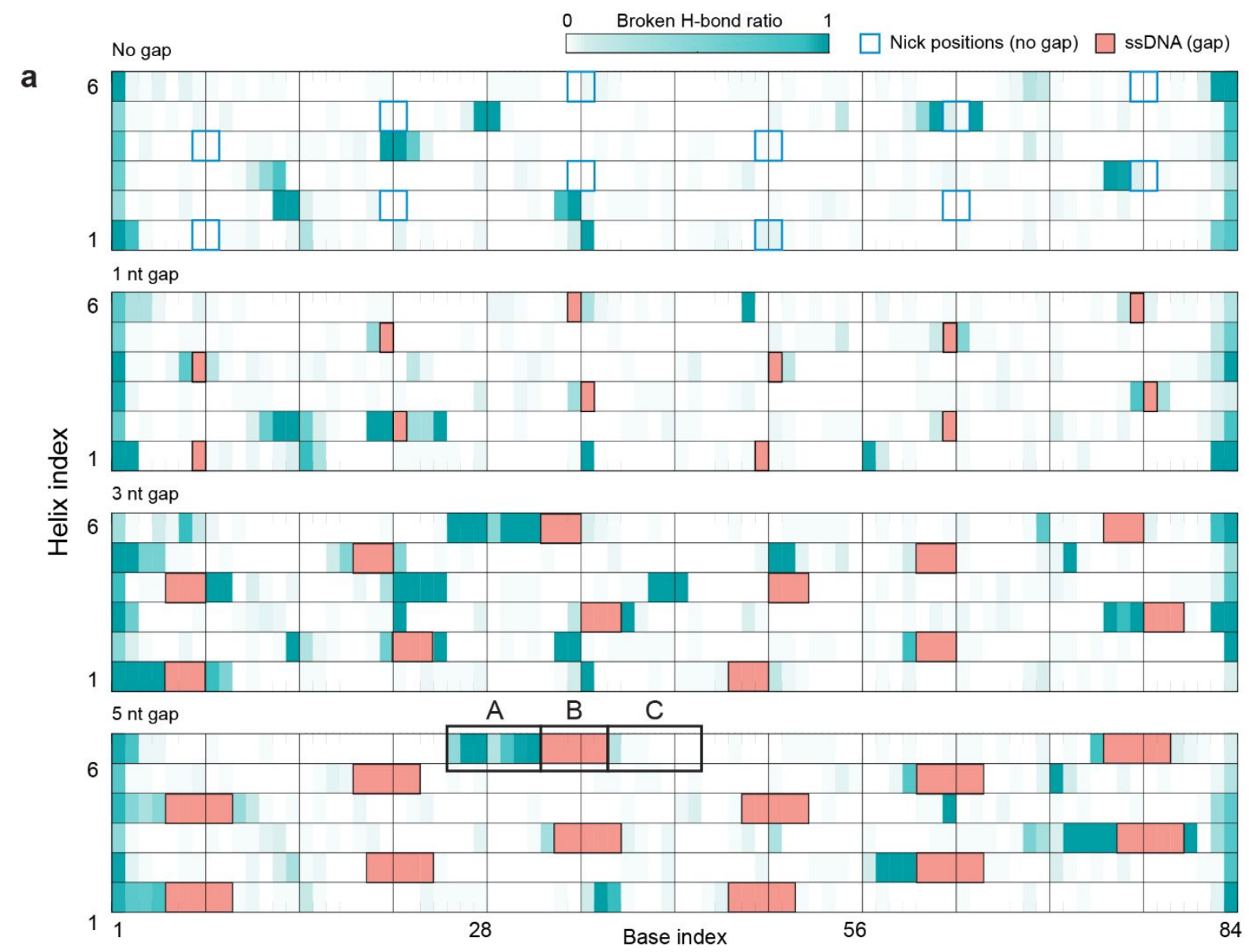

b

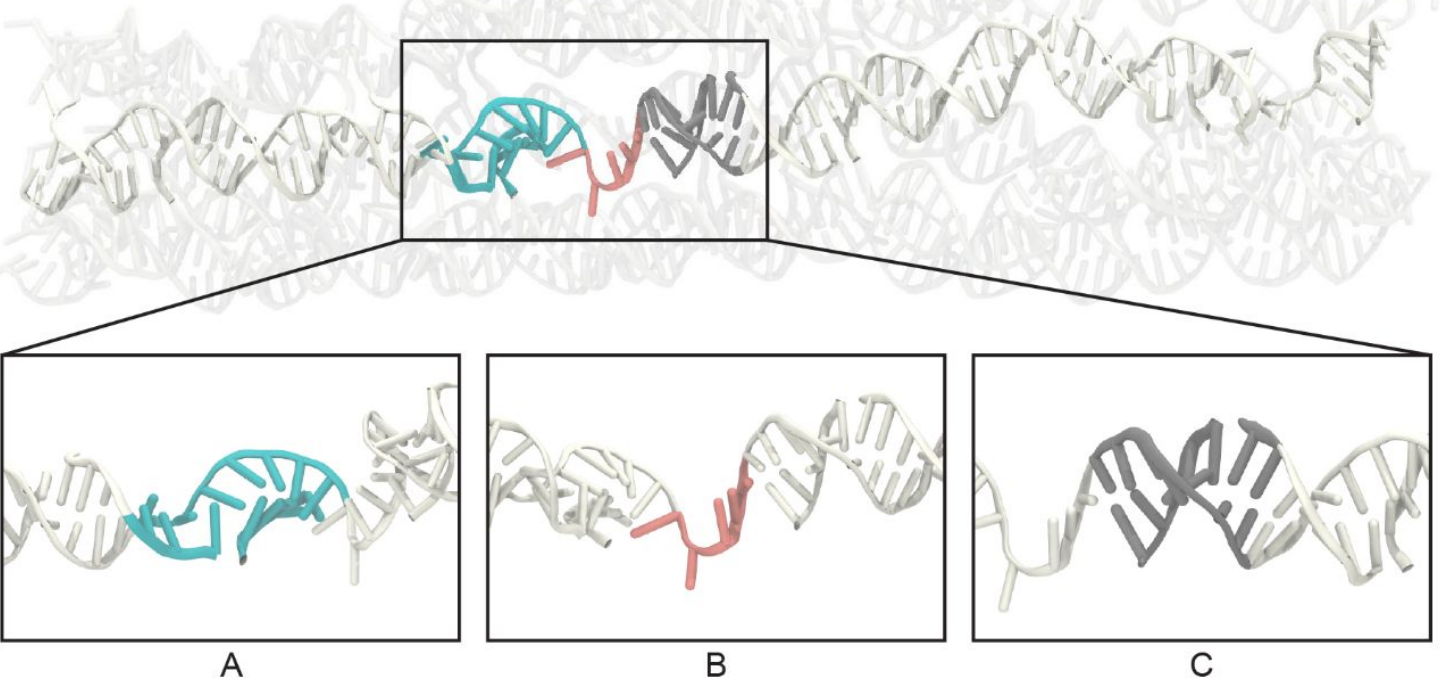

Supplementary Fig. 61. Detailed view of the ssDNA gap and broken dsDNA region in 5-nt-long gap design. (a) Time-average hydrogen bond breakage ratio of all the bases in the non-gapped and the gapped bundles. A, B, and C indicate the representative regions having partially broken bases, ssDNA gap, and well-paired bases, respectively. (b) A snapshot of 5-nt-long gap design in an equilibrium state and a detailed view of the regions as indicated in (a). 


\section{FE simulation on various cross-sections with engineered defects}

a

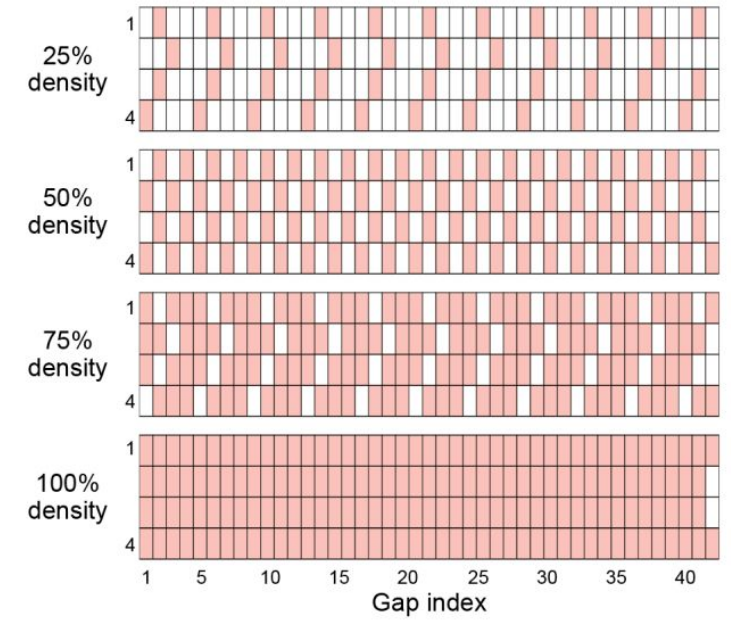

b

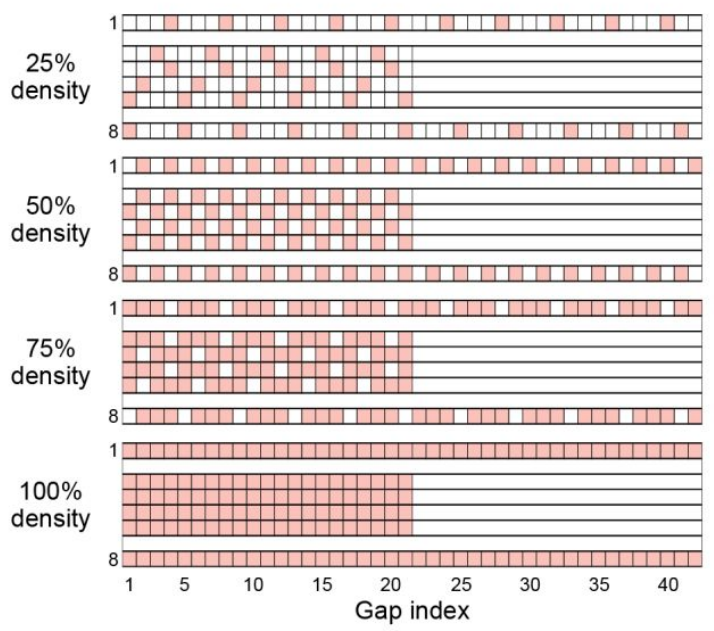

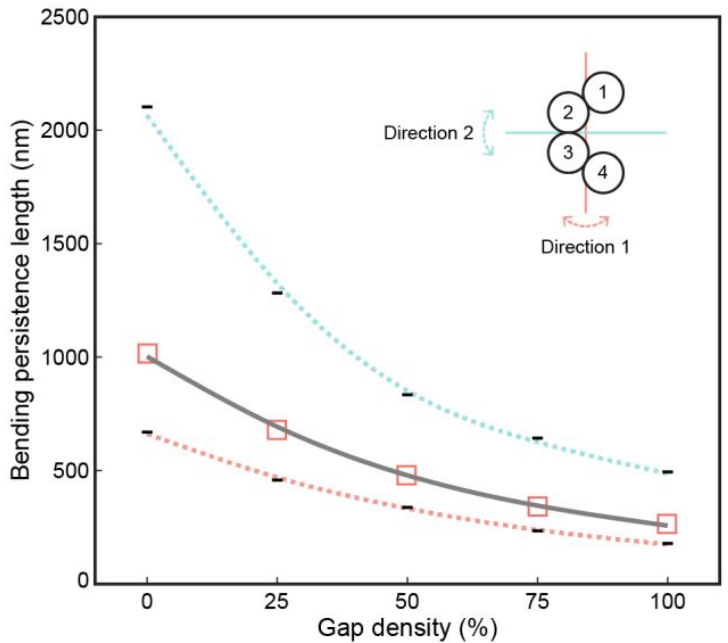

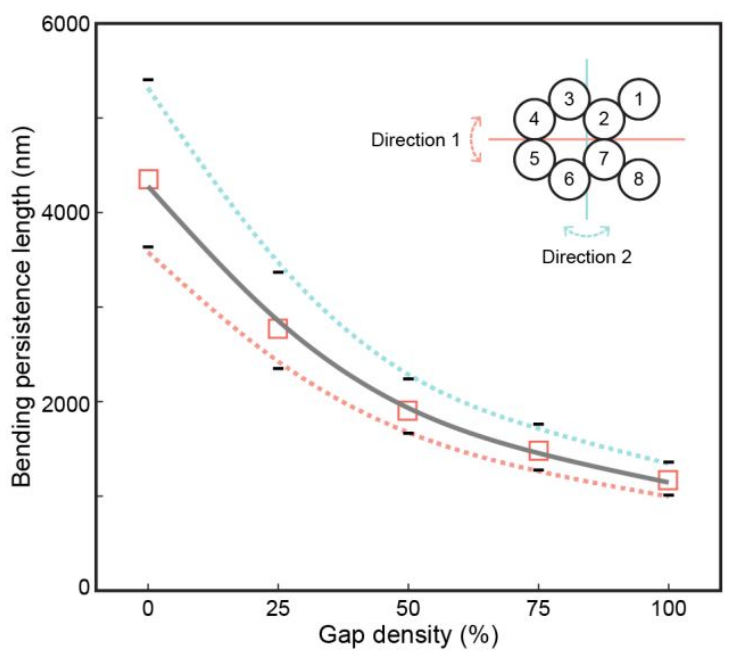

Supplementary Fig. 62. Gap layout and estimated bending persistence length of (a) 4HB-hex and (b) 8HB-hex design. Red colored boxes indicate the location of 5-nt-long gaps. Red and teal dotted lines in the graph are spline-fitted curves of the bending persistence length calculated from two different first bending modes in NMA. Red empty boxes are harmonic mean of the two values and grey solid line is the spline-fitted curve of them. 
a

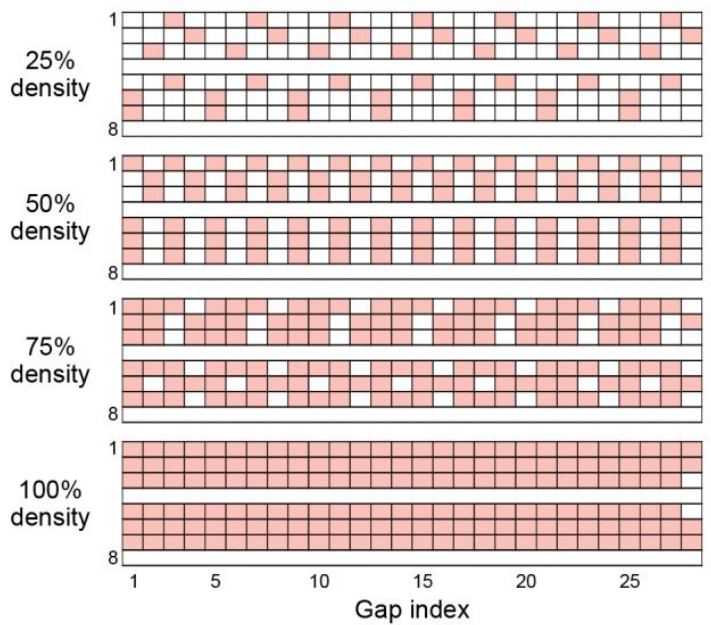

b

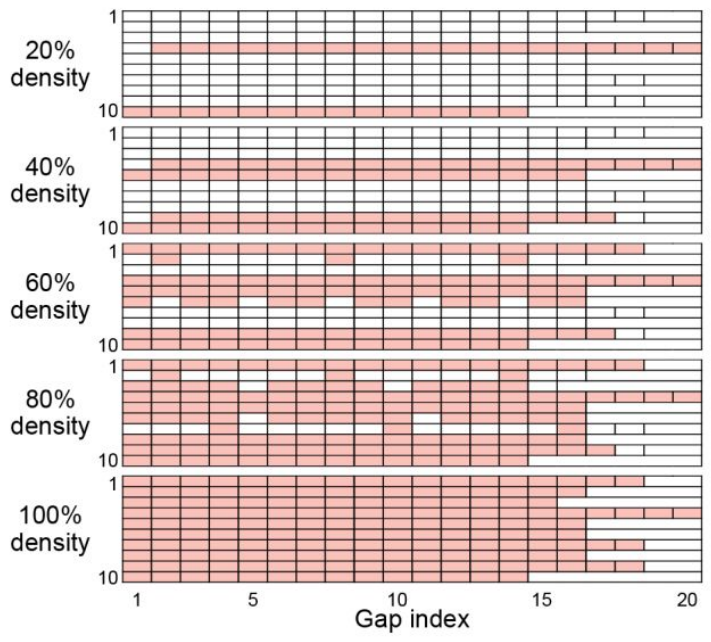

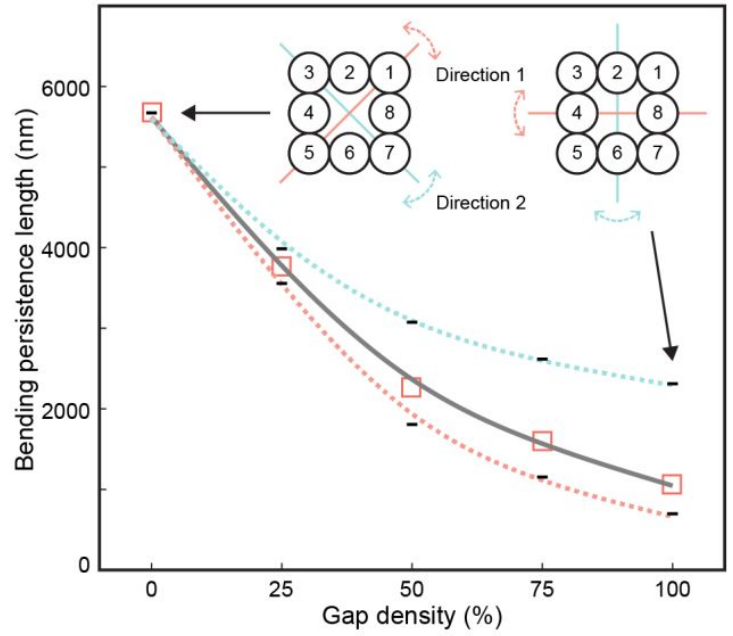

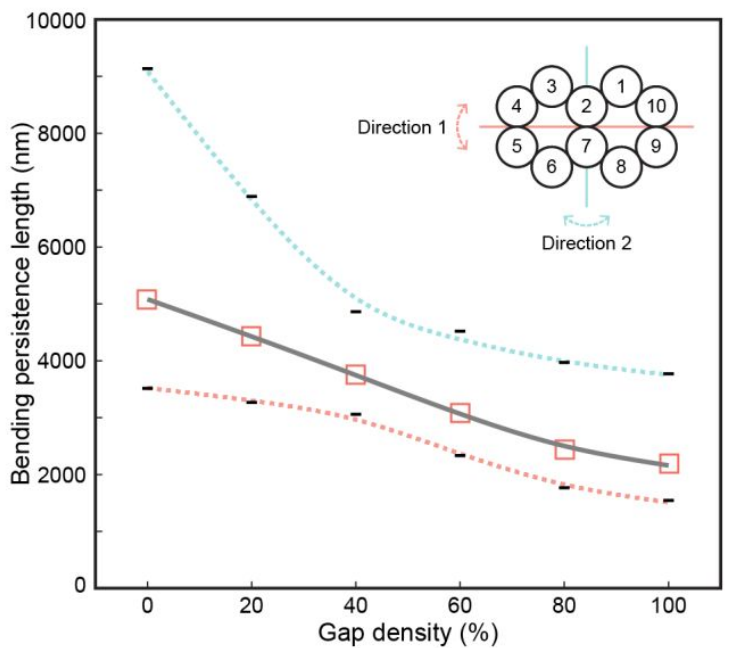

Supplementary Fig. 63. Gap layout and estimated bending persistence length of (a) 8HB-sq and (b) 10HB-hex design. Red colored boxes indicate the location of 5-nt-long gaps. Red and teal dotted lines in the graph are spline-fitted curves of the bending persistence length calculated from two different first bending modes in NMA. Red empty boxes are harmonic mean of the two values and grey solid line is the spline-fitted curve of them. 
a

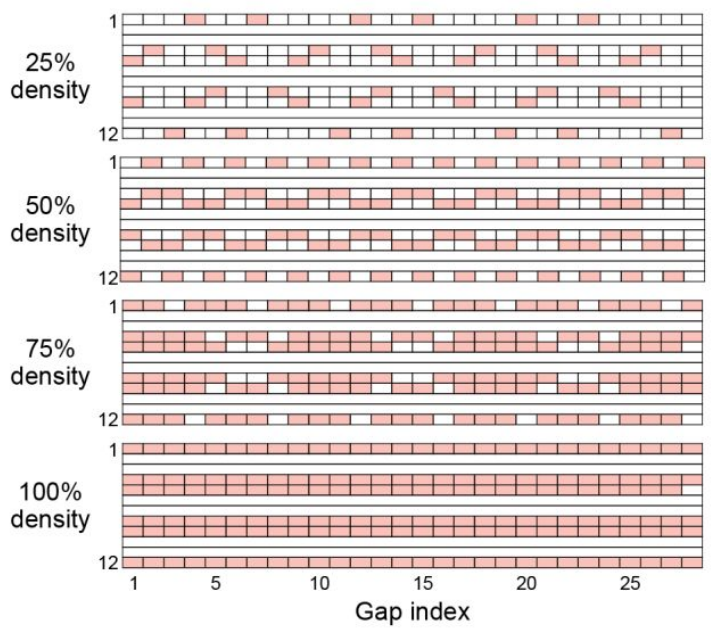

b

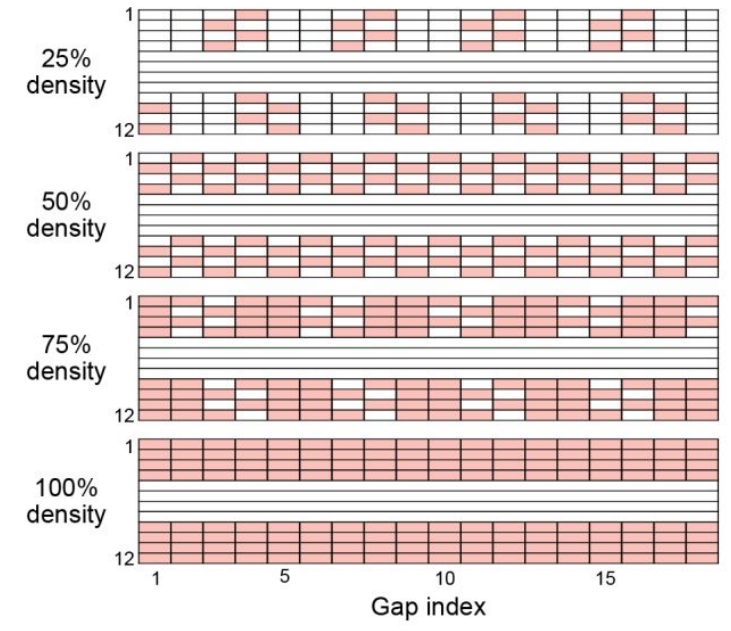

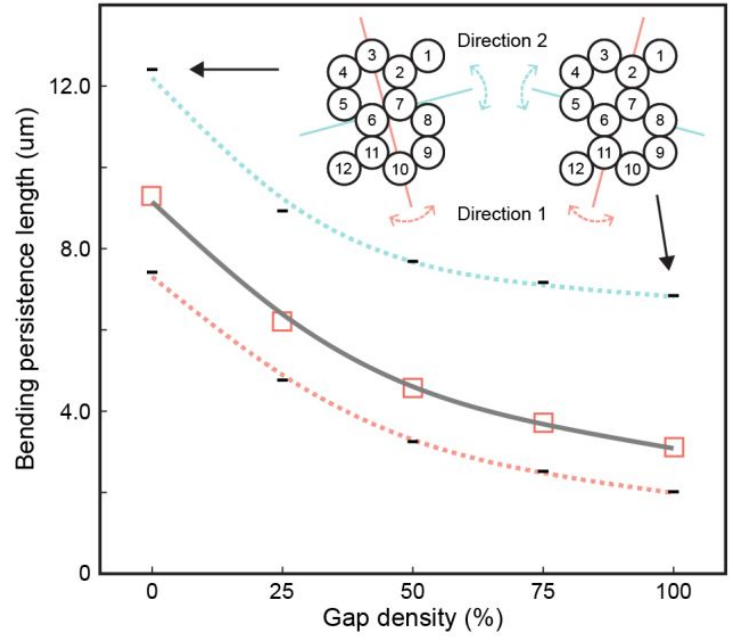

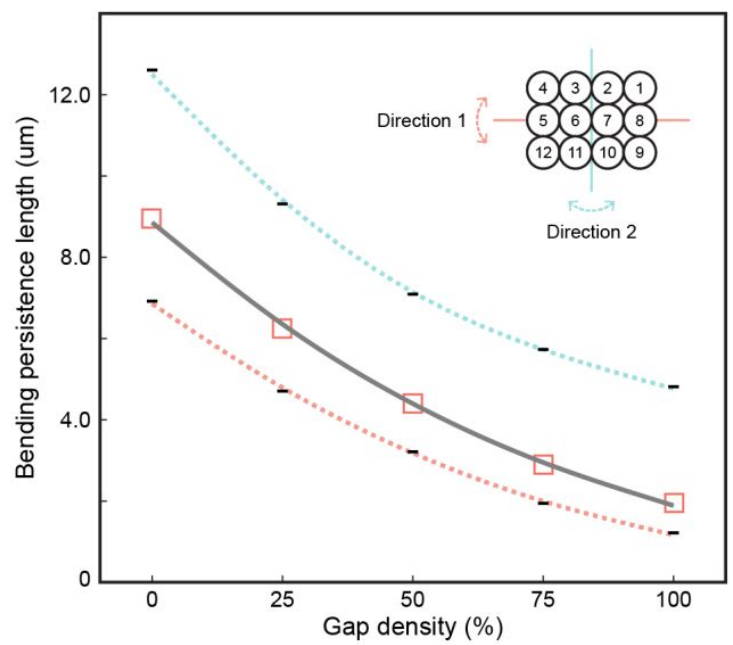

Supplementary Fig. 64. Gap layout and estimated bending persistence length of (a) 12HB-hex and (b) 12HB-sq design. Red colored boxes indicate the location of 5-nt-long gaps. Red and teal dotted lines in the graph are spline-fitted curves of the bending persistence length calculated from two different first bending modes in NMA. Red empty boxes are harmonic mean of the two values and grey solid line is the spline-fitted curve of them. 
a

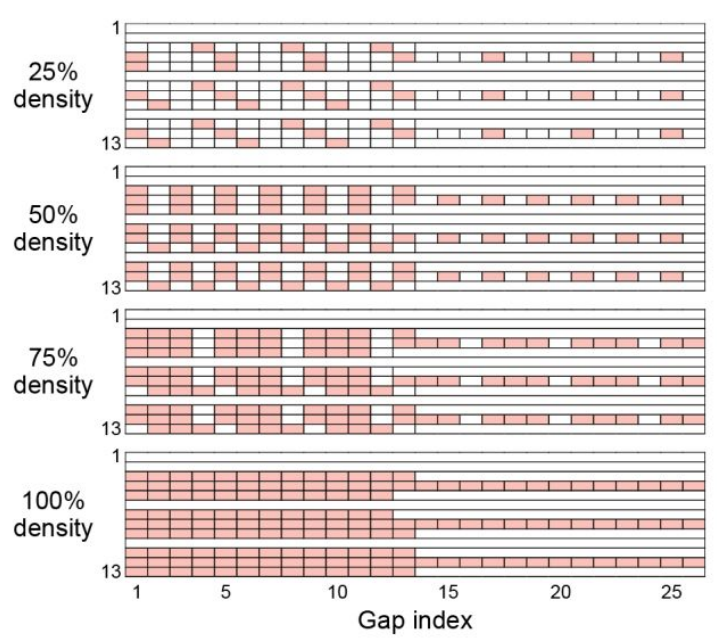

b

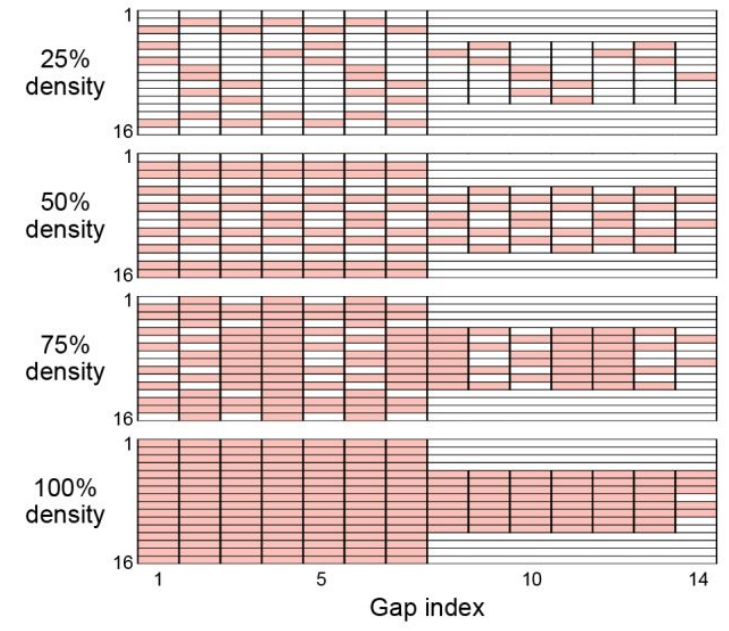

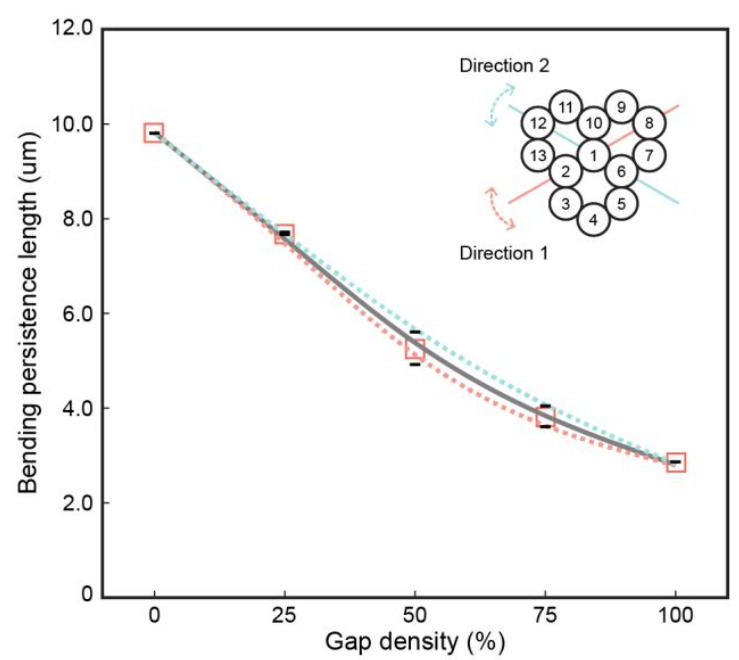

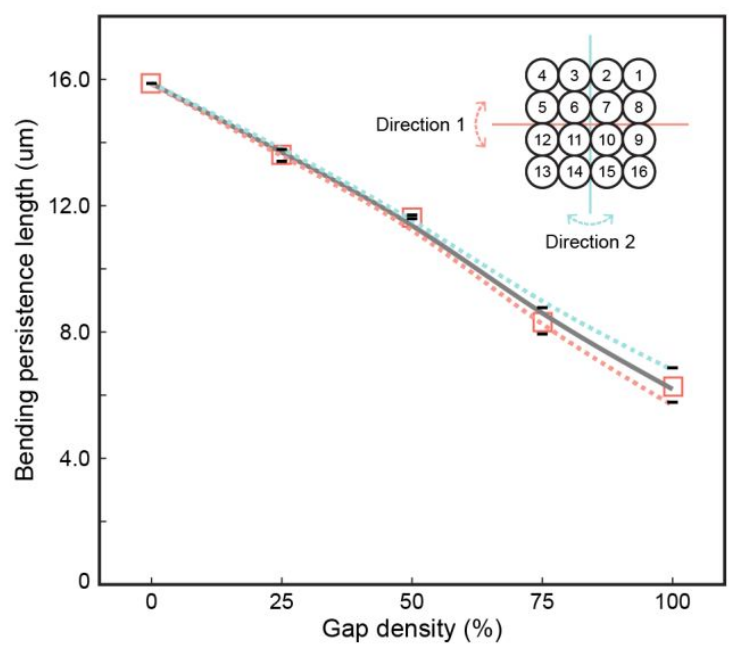

Supplementary Fig. 65. Gap layout and estimated bending persistence length of (a) 13HB-hex and (b) 16HB-sq design. Red colored boxes indicate the location of 5-nt-long gaps. Red and teal dotted lines in the graph are spline-fitted curves of the bending persistence length calculated from two different first bending modes in NMA. Red empty boxes are harmonic mean of the two values and grey solid line is the spline-fitted curve of them. 


\section{Experimental result of $10 \mathrm{HB}$ and $12 \mathrm{HB}$ designs}

10.1. Experimental characterization of $10 \mathrm{HB}$ structures

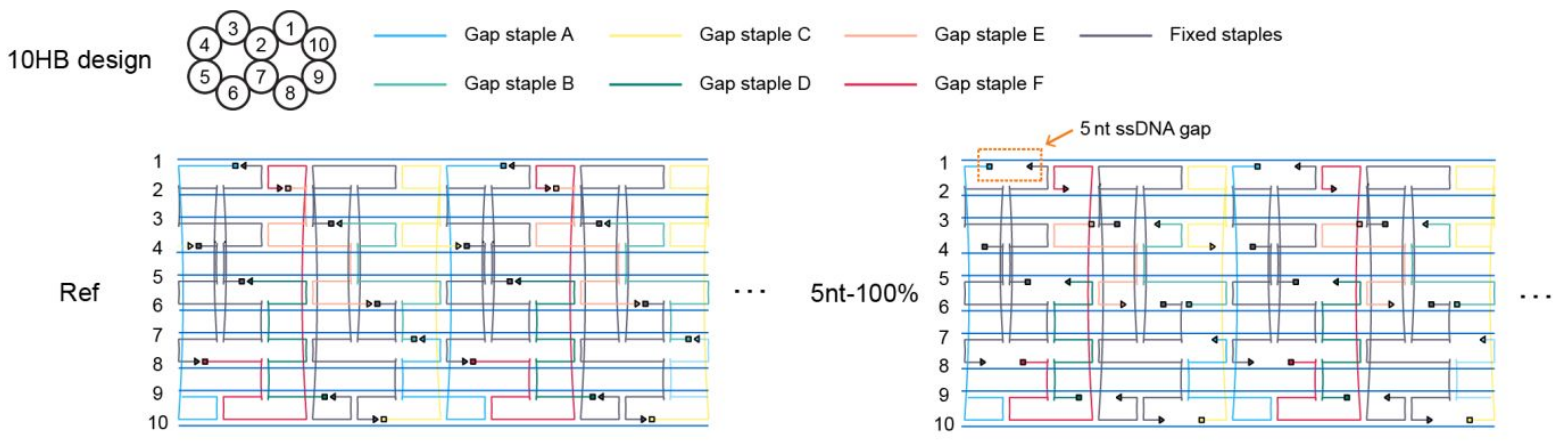

Supplementary Fig. 66. 10HB gap design. (a) Repetitive scaffold and staple route constituting 10HB with honeycomb-lattice packing. Rectangles and triangles indicate the 5' and 3' end of staple DNAs, respectively. Staples were categorized by the location of their nicks, and each group was represented by a different color. Refer to Supplementary Table 7 for all staple sequences. 

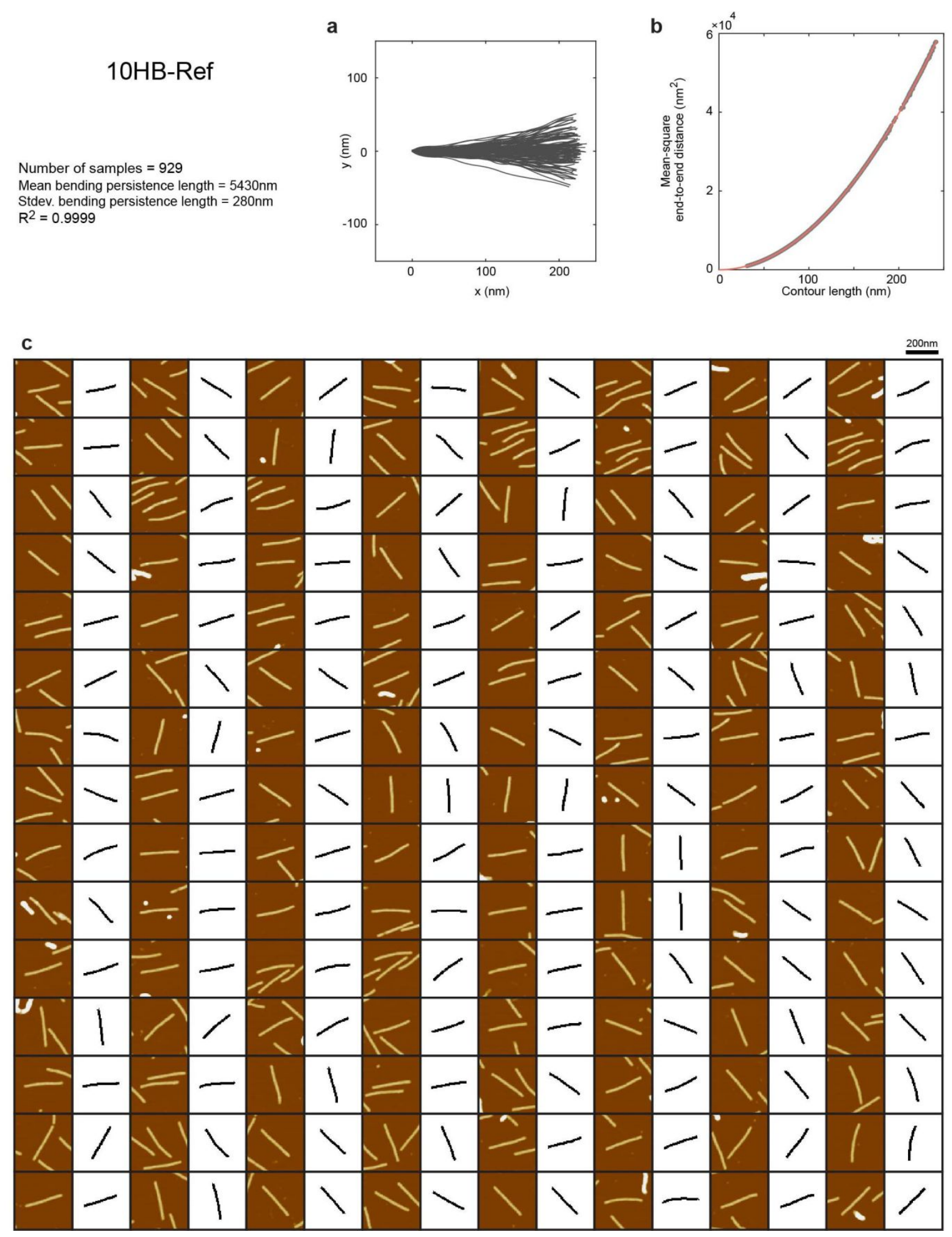

Supplementary Fig. 67. Experimental characterization of 10HB-Ref design. (a) Aligned contour distribution of 120 representative monomers. (b) Calculation of the mean bending persistence length by fitting all measured data. (c) AFM images and extracted monomer contours shown in (a). 
a

10HB-5nt-20\%

Number of samples $=904$

Mean bending persistence length $=4660 \mathrm{~nm}$

Stdev. bending persistence length $=220 \mathrm{~nm}$

$R^{2}=0.9987$
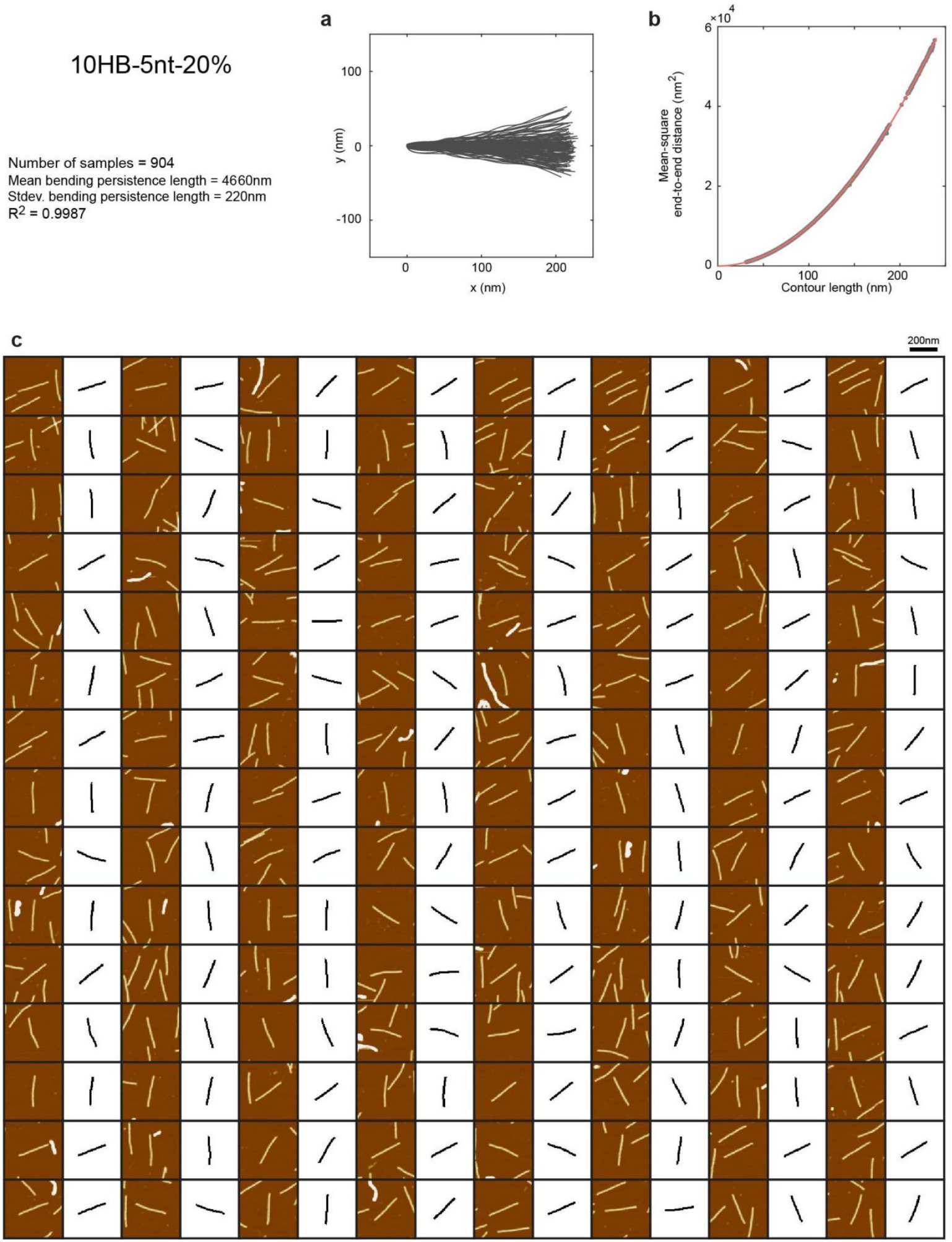

Supplementary Fig. 68. Experimental characterization of 10HB-20\% design. (a) Aligned contour distribution of 120 representative monomers. (b) Calculation of the mean bending persistence length by fitting all measured data. (c) AFM images and extracted monomer contours shown in (a). 

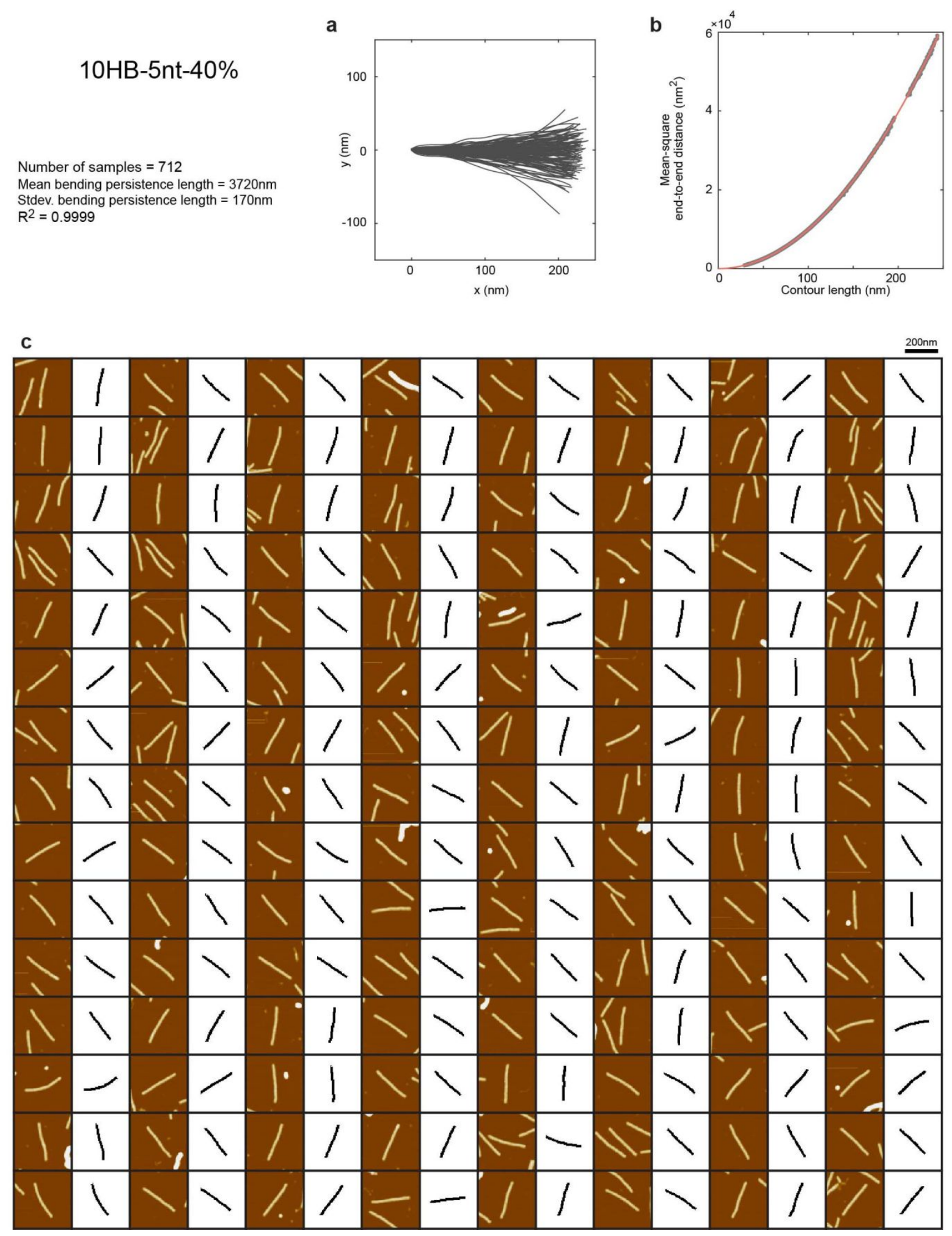

Supplementary Fig. 69. Experimental characterization of 10HB-40\% design. (a) Aligned contour distribution of 120 representative monomers. (b) Calculation of the mean bending persistence length by fitting all measured data. (c) AFM images and extracted monomer contours shown in (a). 
a

10HB-5nt-60\%

Number of samples $=520$

Mean bending persistence length $=3280 \mathrm{~nm}$

Stdev. bending persistence length $=150 \mathrm{~nm}$

$R^{2}=0.9999$
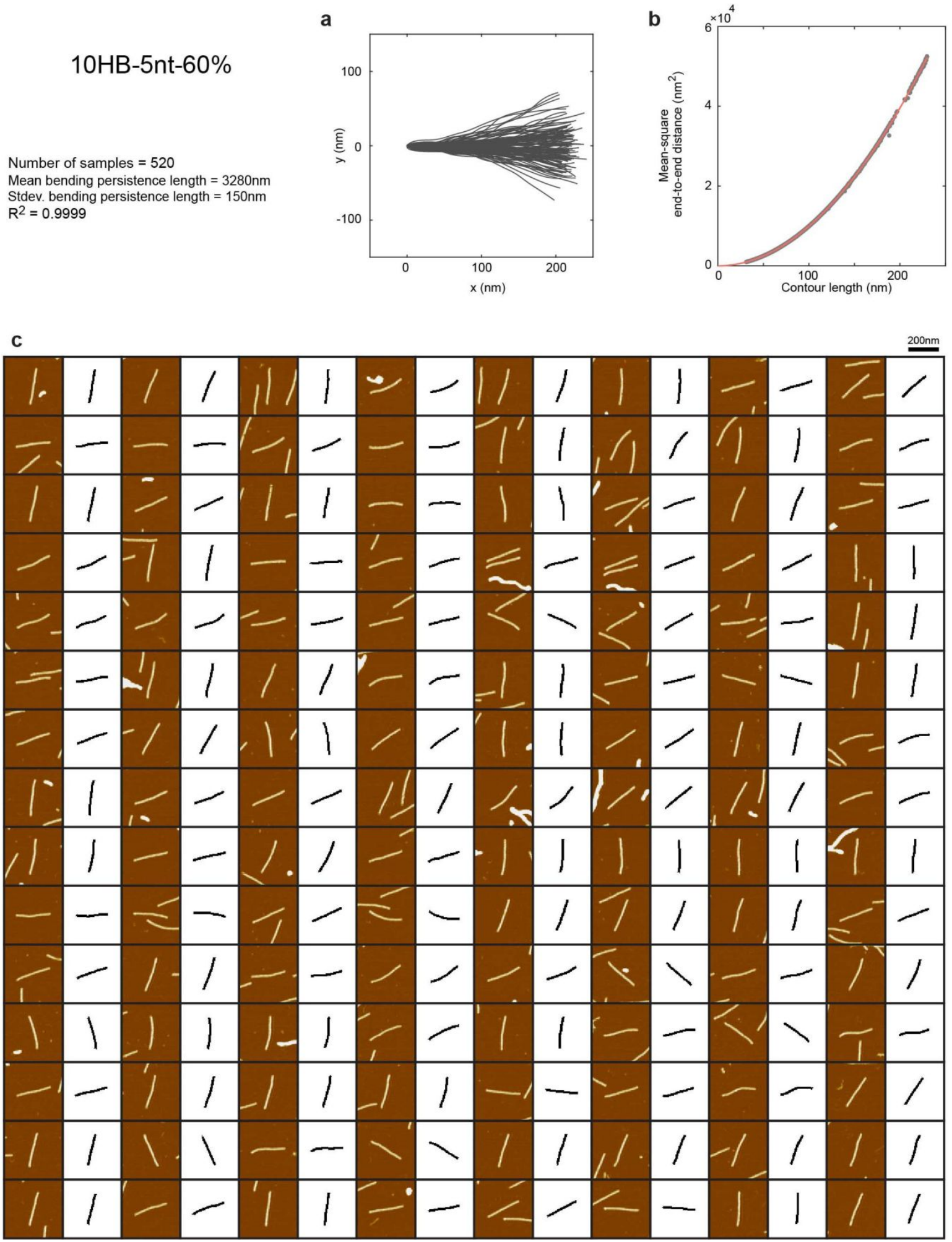

Supplementary Fig. 70. Experimental characterization of 10HB-60\% design. (a) Aligned contour distribution of 120 representative monomers. (b) Calculation of the mean bending persistence length by fitting all measured data. (c) AFM images and extracted monomer contours shown in (a). 

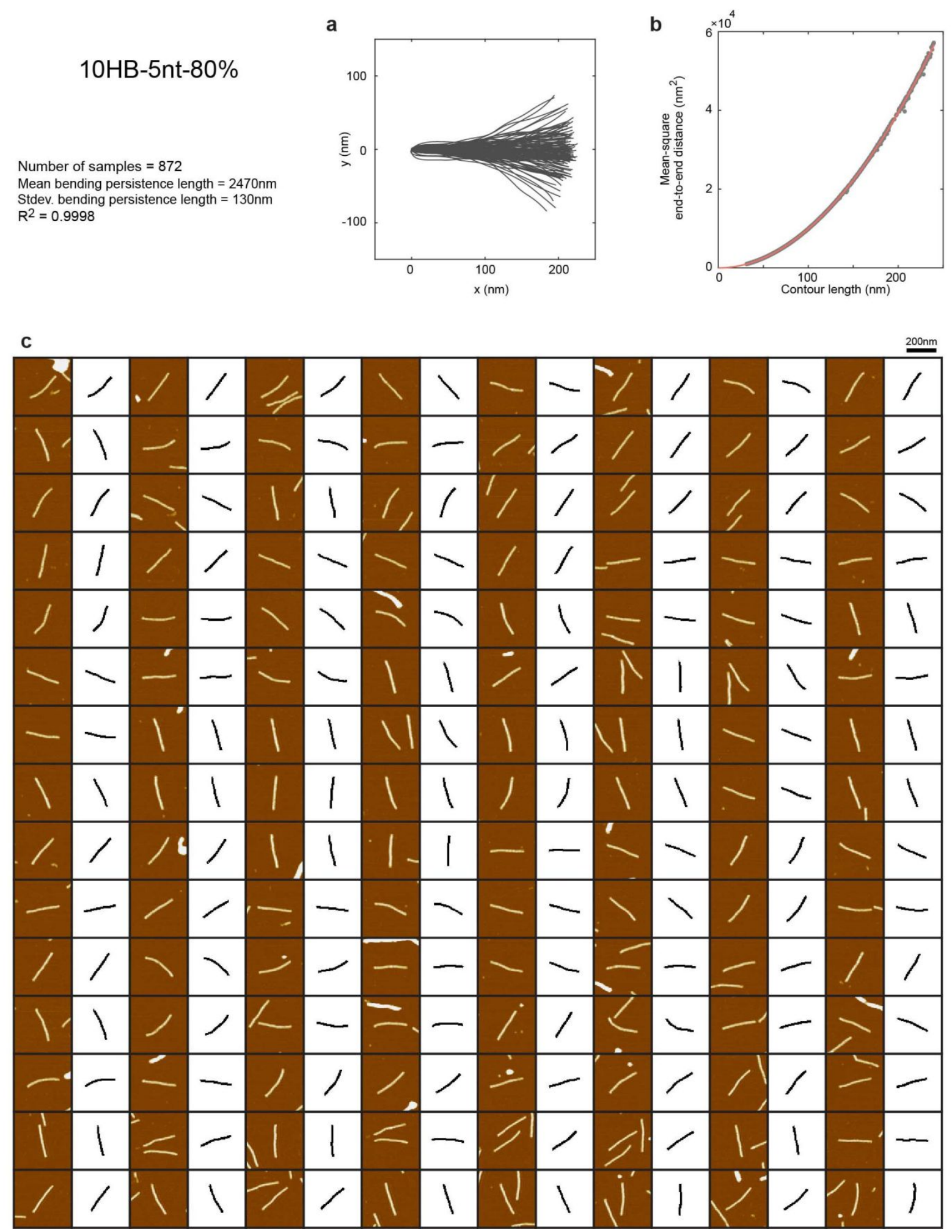

Supplementary Fig. 71. Experimental characterization of 10HB-80\% design. (a) Aligned contour distribution of 120 representative monomers. (b) Calculation of the mean bending persistence length by fitting all measured data. (c) AFM images and extracted monomer contours shown in (a). 
a

10HB-5nt-100\%

Number of samples $=988$

Mean bending persistence length $=2080 \mathrm{~nm}$

Stdev . bending persistence length $=110 \mathrm{~nm}$

$R^{2}=0.9998$
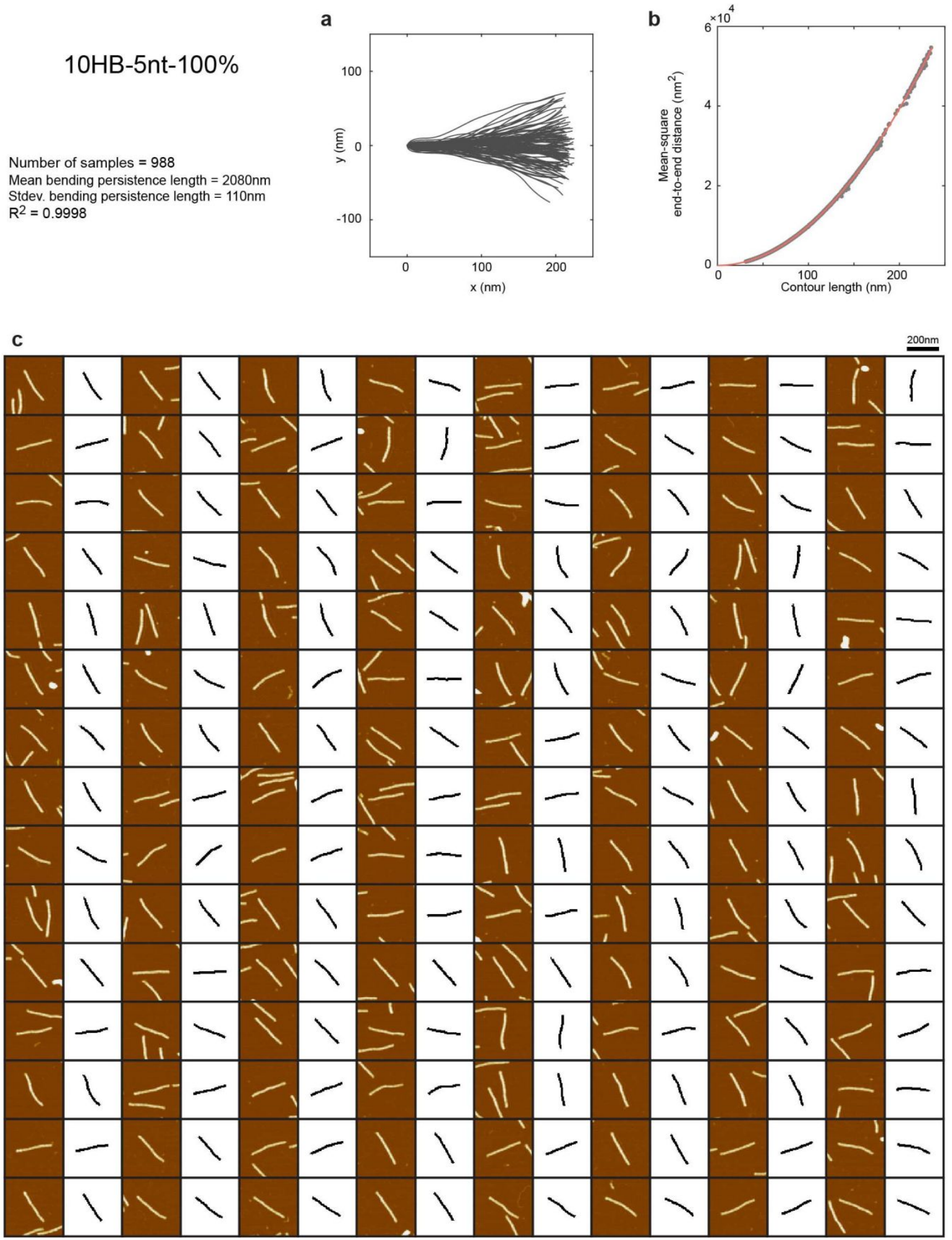

Supplementary Fig. 72. Experimental characterization of 10HB-100\% design. (a) Aligned contour distribution of 120 representative monomers. (b) Calculation of the mean bending persistence length by fitting all measured data. (c) AFM images and extracted monomer contours shown in (a). 


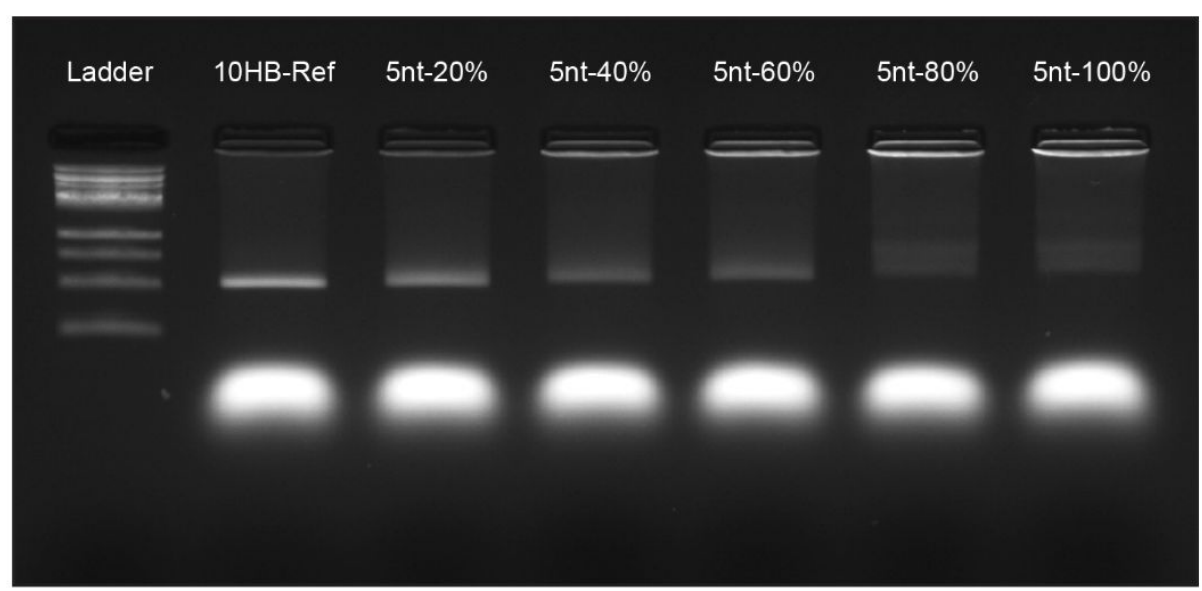

Supplementary Fig. 73. Gel electrophoresis result of 10HB designs. 
10-2. Experimental characterization of $12 \mathrm{HB}$ hinged structures
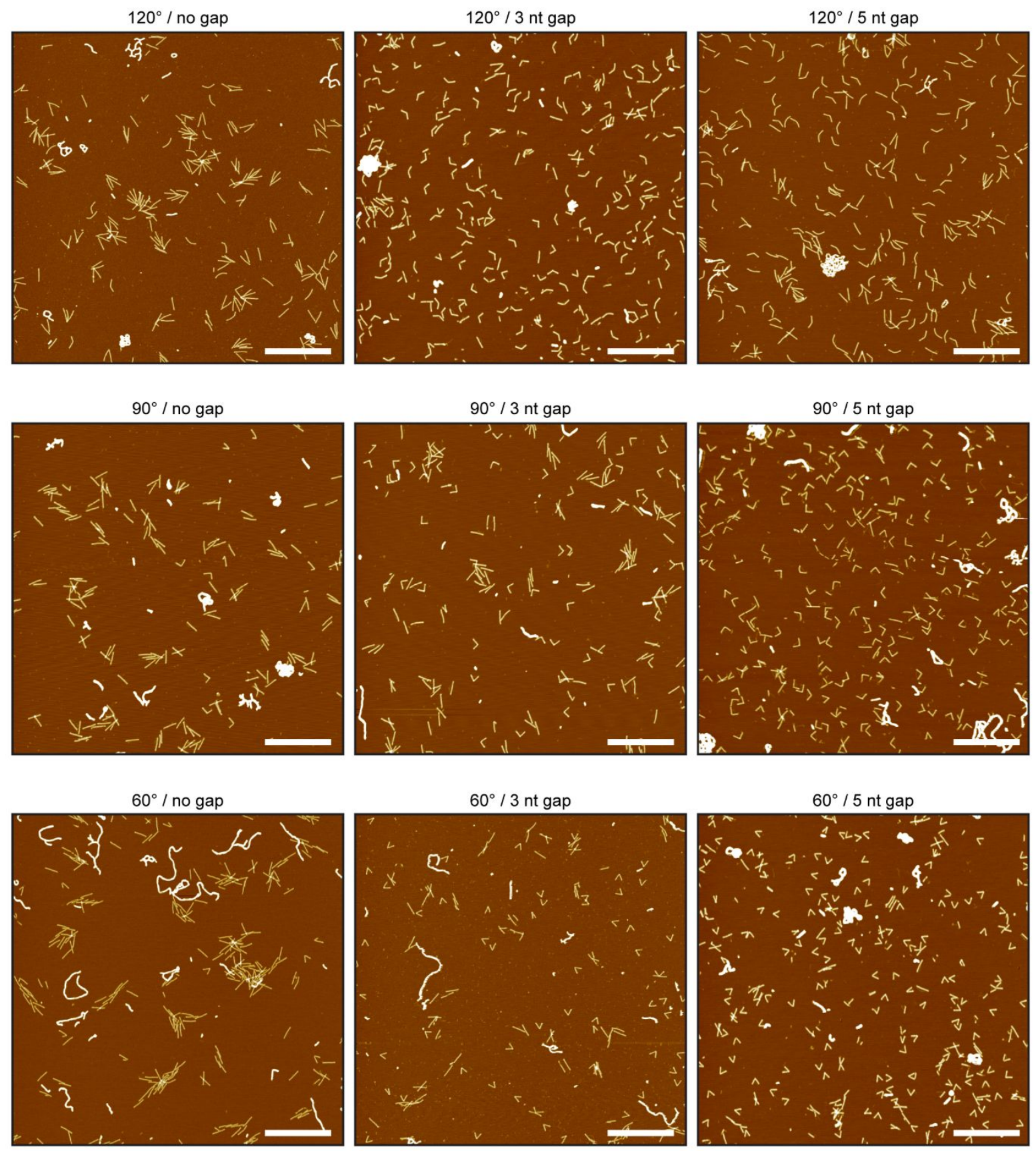

Supplementary Fig. 74. Representative AFM images of 12HB structures with different hinge stiffness and included angles. Scale bars: $1 \mu \mathrm{m}$. 


\section{Supplementary Tables}

11.1. Experimental results

\begin{tabular}{|c|c|c|c|}
\hline Design & $\begin{array}{c}\text { Average bending } \\
\text { persistence length (nm) }\end{array}$ & $\begin{array}{l}\text { Std. deviation of bending } \\
\text { persistence length }(\mathrm{nm})\end{array}$ & $\begin{array}{l}\text { Number of } \\
\text { samples (N) }\end{array}$ \\
\hline 4HB-Ref & 1000 & 50 & 634 \\
\hline 4HB-1nt-25\% & 900 & 40 & 396 \\
\hline 4HB-1nt-50\% & 840 & 40 & 453 \\
\hline 4HB-1nt-75\% & 800 & 40 & 619 \\
\hline 4HB-1nt-100\% & 760 & 40 & 399 \\
\hline 4HB-3nt-25\% & 740 & 40 & 506 \\
\hline 4HB-3nt-50\% & 650 & 30 & 563 \\
\hline 4HB-3nt-75\% & 470 & 20 & 589 \\
\hline 4HB-3nt-100\% & 400 & 20 & 365 \\
\hline 4HB-5nt-25\% & 660 & 30 & 231 \\
\hline 4HB-5nt-50\% & 510 & 20 & 338 \\
\hline 4HB-5nt-75\% & 340 & 10 & 375 \\
\hline 4HB-5nt-100\% & 300 & 10 & 660 \\
\hline 6HB-Ref & 2030 & 80 & 682 \\
\hline 6HB-1nt-17\% & 1830 & 70 & 702 \\
\hline 6HB-1nt-33\% & 1750 & 70 & 504 \\
\hline 6HB-1nt-50\% & 1610 & 60 & 384 \\
\hline 6HB-1nt-67\% & 1540 & 60 & 647 \\
\hline 6HB-1nt-83\% & 1490 & 60 & 643 \\
\hline 6HB-1nt-100\% & 1450 & 90 & 672 \\
\hline 6HB-2nt-100\% & 1040 & 40 & 627 \\
\hline 6HB-3nt-17\% & 1780 & 70 & 442 \\
\hline 6HB-3nt-33\% & 1490 & 80 & 473 \\
\hline $6 H B-3 n t-50 \%$ & 1300 & 50 & 502 \\
\hline 6HB-3nt-67\% & 1170 & 60 & 553 \\
\hline 6HB-3nt-83\% & 1050 & 40 & 401 \\
\hline 6HB-3nt-100\% & 870 & 30 & 438 \\
\hline 6HB-4nt-100\% & 750 & 30 & 418 \\
\hline 6HB-5nt-17\% & 1730 & 80 & 750 \\
\hline 6HB-5nt-33\% & 1420 & 60 & 394 \\
\hline 6HB-5nt-50\% & 1190 & 50 & 665 \\
\hline $6 H B-5 n t-67 \%$ & 930 & 40 & 806 \\
\hline 6HB-5nt-83\% & 820 & 40 & 1073 \\
\hline 6HB-5nt-100\% & 660 & 30 & 779 \\
\hline 6HB-5nt-25\%-Axial & 1190 & 40 & 233 \\
\hline 6HB-5nt-50\%-Axial & 1020 & 50 & 574 \\
\hline 6HB-5nt-75\%-Axial & 900 & 40 & 600 \\
\hline 10HB-Ref & 5430 & 280 & 929 \\
\hline 10HB-5nt-20\% & 4660 & 220 & 904 \\
\hline 10HB-5nt-40\% & 3720 & 170 & 712 \\
\hline 10HB-5nt-60\% & 3280 & 150 & 520 \\
\hline 10HB-5nt-80\% & 2470 & 130 & 872 \\
\hline $10 H B-5 n t-100 \%$ & 2080 & 110 & 988 \\
\hline
\end{tabular}

Supplementary Table. 1. Summary of bending persistence length measurement. 


\begin{tabular}{|c|c|c|c|}
\hline Design & $\begin{array}{c}\text { Number of total } \\
\text { monomers }\end{array}$ & $\begin{array}{c}\text { Number of well-folded } \\
\text { structures }\end{array}$ & $\begin{array}{c}\text { Structural folding } \\
\text { yield }(\%)\end{array}$ \\
\hline 4HB-Ref & 698 & 760 & 91.8 \\
\hline 4HB-1nt-25\% & 420 & 397 & 94.5 \\
\hline 4HB-1nt-50\% & 514 & 470 & 91.4 \\
\hline 4HB-1nt-75\% & 730 & 668 & 91.5 \\
\hline 4HB-1nt-100\% & 494 & 444 & 89.9 \\
\hline 4HB-3nt-25\% & 607 & 541 & 89.1 \\
\hline 4HB-3nt-50\% & 695 & 594 & 85.5 \\
\hline 4HB-3nt-75\% & 694 & 616 & 88.8 \\
\hline 4HB-3nt-100\% & 415 & 379 & 91.3 \\
\hline $4 \mathrm{HB}-5$ nt- $25 \%$ & 333 & 304 & 91.3 \\
\hline 4HB-5nt-50\% & 418 & 362 & 86.6 \\
\hline 4HB-5nt-75\% & 449 & 398 & 88.6 \\
\hline 4HB-5nt-100\% & 798 & 720 & 90.2 \\
\hline 6HB-Ref & 718 & 627 & 87.3 \\
\hline 6HB-1nt-17\% & 531 & 474 & 89.3 \\
\hline 6HB-1nt-33\% & 510 & 454 & 89.0 \\
\hline 6HB-1nt-50\% & 525 & 452 & 86.1 \\
\hline 6HB-1nt-67\% & 611 & 531 & 86.9 \\
\hline 6HB-1nt-83\% & 543 & 490 & 90.2 \\
\hline 6HB-1nt-100\% & 565 & 520 & 92.0 \\
\hline 6HB-1nt-200\% & 483 & 428 & 88.6 \\
\hline 6HB-3nt-17\% & 459 & 392 & 85.4 \\
\hline 6HB-3nt-33\% & 566 & 486 & 85.9 \\
\hline $6 H B-3 n t-50 \%$ & 601 & 536 & 89.2 \\
\hline 6HB-3nt-67\% & 616 & 522 & 84.7 \\
\hline $6 \mathrm{HB}-3 n t-83 \%$ & 731 & 633 & 86.6 \\
\hline 6HB-3nt-100\% & 647 & 531 & 82.1 \\
\hline $6 \mathrm{HB}-4 n t-100 \%$ & 570 & 485 & 85.1 \\
\hline 6HB-5nt-17\% & 541 & 496 & 91.7 \\
\hline 6HB-5nt-33\% & 584 & 508 & 87.0 \\
\hline 6HB-5nt-50\% & 609 & 538 & 88.3 \\
\hline 6HB-5nt-67\% & 489 & 370 & 75.7 \\
\hline 6HB-5nt-83\% & 600 & 515 & 85.8 \\
\hline 6HB-5nt-100\% & 494 & 406 & 82.2 \\
\hline 6HB-5nt-25\%-Axial & 606 & 491 & 81.0 \\
\hline 6HB-5nt-50\%-Axial & 645 & 542 & 84.0 \\
\hline 6HB-5nt-75\%-Axial & 592 & 504 & 85.1 \\
\hline 10HB-Ref & 999 & 939 & 94.0 \\
\hline 10HB-5nt-20\% & 1109 & 976 & 88.0 \\
\hline 10HB-5nt-40\% & 873 & 748 & 85.7 \\
\hline 10HB-5nt-60\% & 708 & 577 & 81.5 \\
\hline 10HB-5nt-80\% & 1101 & 883 & 80.2 \\
\hline 10HB-5nt-100\% & 852 & 617 & 72.4 \\
\hline
\end{tabular}

Supplementary Table 2. Structural folding yield result. At least five AFM images were used to analyze the structural folding yield of each case. 


\begin{tabular}{|c|c|c|c|}
\hline \multicolumn{4}{|c|}{ Interhelix distance (nm) } \\
\hline Honeycomb lattice & 2.25 & Square lattice & 2.5 \\
\hline \multicolumn{4}{|c|}{ Mechanical properties } \\
\hline & $\mathrm{EA}_{\mathrm{dsDNA}}(\mathrm{pN})$ & $\mathrm{EI}_{\mathrm{dsDNA}}\left(\mathrm{pN} \cdot \mathrm{nm}^{2}\right)$ & $\mathrm{GJ}_{\mathrm{dsDNA}}\left(\mathrm{pN} \cdot \mathrm{nm}^{2}\right)$ \\
\hline dsDNA element & 1100 & 230 & 460 \\
\hline \multicolumn{4}{|c|}{ Normalized rigidity factor (with respect to dsDNA element) } \\
\hline & $\mathrm{EA} / \mathrm{EA}_{\mathrm{dsDNA}}$ & $\mathrm{EI} / \mathrm{EI}_{\mathrm{dsDNA}}$ & $\mathrm{GJ} / \mathrm{GJ}_{\mathrm{dsDNA}}$ \\
\hline Crossover element & 1.0 & 0.2 & 0.1 \\
\hline HJ core element & 0.069 & 0.117 & 1.0 \\
\hline Nick element & 1.0 & 1.0 & 1.0 \\
\hline 1-nt ssDNA gap element & 0.054 & 0.01 & 0.01 \\
\hline 2-nt ssDNA gap element & 0.035 & 0.009 & 0.01 \\
\hline 3-nt ssDNA gap element & 0.017 & 0.009 & 0.01 \\
\hline 4-nt ssDNA gap element & 0.014 & 0.009 & 0.01 \\
\hline 5-nt ssDNA gap element & 0.011 & 0.009 & 0.01 \\
\hline
\end{tabular}

Supplementary Table 3. Parameters used in FE modeling. 


\begin{tabular}{|c|c|c|c|c|c|c|c|c|c|}
\hline Name & $\begin{array}{l}\text { Base } \\
\text { count }\end{array}$ & $\begin{array}{c}\text { Molecular } \\
\text { weight } \\
\text { (theoretical) } \\
\text { [Da] }\end{array}$ & $\begin{array}{c}\text { Molecular } \\
\text { weight } \\
\text { (MALDI- } \\
\text { TOF) } \\
\text { [Da] }\end{array}$ & Difference & Name & $\begin{array}{l}\text { Base } \\
\text { count }\end{array}$ & $\begin{array}{c}\text { Molecular } \\
\text { weight } \\
\text { (theoretical) } \\
\text { [Da] }\end{array}$ & $\begin{array}{c}\text { Molecular } \\
\text { weight } \\
\text { (MALDI- } \\
\text { TOF) } \\
\text { [Da] }\end{array}$ & Difference \\
\hline 4hb_nogap_A01 & 48 & 14768.64 & 14789.0 & $0.14 \%$ & 4hb_5gap_A01 & 38 & 11768.7 & 11788.0 & $0.16 \%$ \\
\hline 4hb_nogap_A02 & 48 & 14784.7 & 14821.0 & $0.25 \%$ & 4hb_5gap_A02 & 38 & 11729.71 & 11744.0 & $0.12 \%$ \\
\hline 4hb_nogap_A03 & 48 & 14654.63 & 14699.0 & $0.30 \%$ & 4hb_5gap_A03 & 38 & 11566.6 & 11571.0 & $0.04 \%$ \\
\hline 4hb_nogap_A04 & 48 & 14829.76 & 14877.0 & $0.32 \%$ & 4hb_5gap_A04 & 38 & 11682.7 & 11719.0 & $0.31 \%$ \\
\hline 4hb_nogap_A05 & 48 & 14950.83 & 15002.0 & $0.34 \%$ & 4hb_5gap_A05 & 38 & 11764.78 & 11783.0 & $0.15 \%$ \\
\hline 4hb_nogap_A06 & 48 & 14661.55 & 14697.0 & $0.24 \%$ & 4hb_5gap_A06 & 38 & 11612.57 & 11646.0 & $0.29 \%$ \\
\hline 4hb_nogap_A07 & 48 & 14744.61 & 14797.0 & $0.36 \%$ & 4hb_5gap_A07 & 38 & 11694.65 & 11714.0 & $0.17 \%$ \\
\hline 4hb_nogap_A08 & 48 & 14698.57 & 14742.0 & $0.30 \%$ & 4hb_5gap_A08 & 38 & 11593.56 & 11611.0 & $0.15 \%$ \\
\hline 4hb_nogap_A09 & 48 & 14804.56 & 14848.0 & $0.29 \%$ & 4hb_5gap_A09 & 38 & 11760.61 & 11785.0 & $0.21 \%$ \\
\hline 4hb_nogap_A10 & 48 & 14840.67 & 14878.0 & $0.25 \%$ & 4hb_5gap_A10 & 38 & 11729.65 & 11756.0 & $0.22 \%$ \\
\hline 4hb_nogap_A11 & 48 & 14523.38 & 14541.0 & $0.12 \%$ & 4hb_5gap_A11 & 38 & 11467.41 & 11482.0 & $0.13 \%$ \\
\hline 4hb_nogap_A12 & 48 & 14818.6 & 14829.0 & $0.07 \%$ & 4hb_5gap_A12 & 38 & 11688.58 & 11701.0 & $0.11 \%$ \\
\hline 4hb_nogap_A13 & 48 & 14548.44 & 14577.0 & $0.20 \%$ & 4hb_5gap_A13 & 38 & 11553.53 & 11579.0 & $0.22 \%$ \\
\hline 4hb_nogap_A14 & 48 & 14844.66 & 14872.0 & $0.18 \%$ & 4hb_5gap_A14 & 38 & 11729.66 & 11738.0 & $0.07 \%$ \\
\hline 4hb_nogap_A15 & 48 & 14685.64 & 14710.0 & $0.17 \%$ & 4hb_5gap_A15 & 38 & 11588.6 & 11612.0 & $0.20 \%$ \\
\hline 4hb_nogap_A16 & 48 & 14754.67 & 14774.0 & $0.13 \%$ & 4hb_5gap_A16 & 38 & 11665.66 & 11698.0 & $0.28 \%$ \\
\hline 4hb_nogap_A17 & 48 & 14875.74 & 14915.0 & $0.26 \%$ & 4hb_5gap_A17 & 38 & 11744.68 & 11748.0 & $0.03 \%$ \\
\hline 4hb_nogap_A18 & 48 & 14784.65 & 14816.0 & $0.21 \%$ & 4hb_5gap_A18 & 38 & 11632.62 & 11671.0 & $0.33 \%$ \\
\hline 4hb_nogap_A19 & 36 & 11001.17 & 11018.0 & $0.15 \%$ & 4hb_5gap_A19 & 31 & 9445.17 & 9461.0 & $0.17 \%$ \\
\hline 4hb_nogap_B01 & 36 & 11022.19 & 11039.0 & $0.15 \%$ & 4hb_5gap_B01 & 31 & 9482.19 & 9512.0 & $0.31 \%$ \\
\hline 4hb_nogap_B02 & 48 & 14657.57 & 14687.0 & $0.20 \%$ & 4hb_5gap_B02 & 38 & 11617.6 & 11641.0 & $0.20 \%$ \\
\hline 4hb_nogap_B03 & 48 & 14690.61 & 14709.0 & $0.13 \%$ & 4hb_5gap_B03 & 38 & 11603.56 & 11603.0 & $0.00 \%$ \\
\hline 4hb_nogap_B04 & 48 & 14962.85 & 14990.0 & $0.18 \%$ & 4hb_5gap_B04 & 38 & 11849.81 & 11873.0 & $0.20 \%$ \\
\hline 4hb_nogap_B05 & 48 & 14928.82 & 14980.0 & $0.34 \%$ & 4hb_5gap_B05 & 38 & 11886.83 & 11894.0 & $0.06 \%$ \\
\hline 4hb_nogap_B06 & 48 & 14771.7 & 14820.0 & $0.33 \%$ & 4hb_5gap_B06 & 38 & 11680.67 & 11707.0 & $0.23 \%$ \\
\hline 4hb_nogap_B07 & 48 & 14625.5 & 14653.0 & $0.19 \%$ & 4hb_5gap_B07 & 38 & 11543.48 & 11562.0 & $0.16 \%$ \\
\hline 4hb_nogap_B08 & 48 & 14622.5 & 14640.0 & $0.12 \%$ & 4hb_5gap_B08 & 38 & 11579.53 & 11611.0 & $0.27 \%$ \\
\hline 4hb_nogap_B09 & 48 & 14910.67 & 14950.0 & $0.26 \%$ & 4hb_5gap_B09 & 38 & 11746.63 & 11767.0 & $0.17 \%$ \\
\hline 4hb_nogap_B10 & 48 & 14856.61 & 14896.0 & $0.27 \%$ & 4hb_5gap_B10 & 38 & 11772.63 & 11802.0 & $0.25 \%$ \\
\hline 4hb_nogap_B11 & 48 & 14801.63 & 14828.0 & $0.18 \%$ & 4hb_5gap_B11 & 38 & 11771.65 & 11801.0 & $0.25 \%$ \\
\hline 4hb_nogap_B12 & 48 & 14628.45 & 14675.0 & $0.32 \%$ & 4hb_5gap_B12 & 38 & 11587.5 & 11585.0 & $-0.02 \%$ \\
\hline 4hb_nogap_B13 & 48 & 14851.71 & 14901.0 & $0.33 \%$ & 4hb_5gap_B13 & 38 & 11715.68 & 11739.0 & $0.20 \%$ \\
\hline 4hb_nogap_B14 & 48 & 15001.89 & 15055.0 & $0.35 \%$ & 4hb_5gap_B14 & 38 & 11861.82 & 11870.0 & $0.07 \%$ \\
\hline 4hb_nogap_B15 & 48 & 14813.7 & 14862.0 & $0.33 \%$ & 4hb_5gap_B15 & 38 & 11739.71 & 11741.0 & $0.01 \%$ \\
\hline 4hb_nogap_B16 & 48 & 14919.81 & 14959.0 & $0.26 \%$ & 4hb_5gap_B16 & 38 & 11774.77 & 11798.0 & $0.20 \%$ \\
\hline 4hb_nogap_B17 & 48 & 14667.62 & 14714.0 & $0.32 \%$ & 4hb_5gap_B17 & 38 & 11594.61 & 11604.0 & $0.08 \%$ \\
\hline 4hb_nogap_B18 & 48 & 14815.66 & 14867.0 & $0.35 \%$ & 4hb_5gap_B18 & 38 & 11757.67 & 11759.0 & $0.01 \%$ \\
\hline 4hb_nogap_B19 & 36 & 11030.22 & 11054.0 & $0.22 \%$ & 4hb_5gap_B19 & 31 & 9432.17 & 9434.0 & $0.02 \%$ \\
\hline 4hb_nogap_B20 & 40 & 12285.02 & 12307.0 & $0.18 \%$ & 4hb_5gap_B20 & 35 & 10735.03 & 10753.0 & $0.17 \%$ \\
\hline 4hb_nogap_C01 & 40 & 12247.02 & 12276.0 & $0.24 \%$ & 4hb_5gap_C01 & 35 & 10698.01 & 10719.0 & $0.20 \%$ \\
\hline 4hb_nogap_C02 & 48 & 14851.77 & 14905.0 & $0.36 \%$ & 4hb_5gap_C02 & 38 & 11778.76 & 11802.0 & $0.20 \%$ \\
\hline 4hb_nogap_C03 & 48 & 14803.73 & 14852.0 & $0.33 \%$ & 4hb_5gap_C03 & 38 & 11789.79 & 11832.0 & $0.36 \%$ \\
\hline 4hb_nogap_C04 & 48 & 14855.75 & 14891.0 & $0.24 \%$ & 4hb_5gap_C04 & 38 & 11793.77 & 11809.0 & $0.13 \%$ \\
\hline
\end{tabular}




\begin{tabular}{|c|c|c|c|c|c|c|c|c|c|}
\hline 4hb_nogap_C05 & 48 & 14850.79 & 14872.0 & $0.14 \%$ & 4hb_5gap_C05 & 38 & 11728.74 & 11733.0 & $0.04 \%$ \\
\hline 4hb_nogap_C06 & 48 & 14647.57 & 14667.0 & $0.13 \%$ & 4hb_5gap_C06 & 38 & 11597.61 & 11616.0 & $0.16 \%$ \\
\hline 4hb_nogap_C07 & 48 & 14819.71 & 14847.0 & $0.18 \%$ & 4hb_5gap_C07 & 38 & 11697.66 & 11716.0 & $0.16 \%$ \\
\hline 4hb_nogap_C08 & 48 & 14694.59 & 14733.0 & $0.26 \%$ & 4hb_5gap_C08 & 38 & 11547.53 & 11572.0 & $0.21 \%$ \\
\hline 4hb_nogap_C09 & 48 & 14824.66 & 14860.0 & $0.24 \%$ & 4hb_5gap_C09 & 38 & 11668.59 & 11678.0 & $0.08 \%$ \\
\hline 4hb_nogap_C10 & 48 & 14854.65 & 14878.0 & $0.16 \%$ & 4hb_5gap_C10 & 38 & 11699.62 & 11722.0 & $0.19 \%$ \\
\hline 4hb_nogap_C11 & 48 & 14787.58 & 14823.0 & $0.24 \%$ & 4hb_5gap_C11 & 38 & 11712.61 & 11732.0 & $0.17 \%$ \\
\hline 4hb_nogap_C12 & 48 & 14663.51 & 14686.0 & $0.15 \%$ & 4hb_5gap_C12 & 38 & 11548.51 & 11563.0 & $0.13 \%$ \\
\hline 4hb_nogap_C13 & 48 & 14900.69 & 14956.0 & $0.37 \%$ & 4hb_5gap_C13 & 38 & 11804.69 & 11803.0 & $-0.01 \%$ \\
\hline 4hb_nogap_C14 & 48 & 14844.65 & 14885.0 & $0.27 \%$ & 4hb_5gap_C14 & 38 & 11760.67 & 11787.0 & $0.22 \%$ \\
\hline 4hb_nogap_C15 & 48 & 14683.62 & 14723.0 & $0.27 \%$ & 4hb_5gap_C15 & 38 & 11613.61 & 11616.0 & $0.02 \%$ \\
\hline 4hb_nogap_C16 & 48 & 14834.73 & 14870.0 & $0.24 \%$ & 4hb_5gap_C16 & 38 & 11746.7 & 11778.0 & $0.27 \%$ \\
\hline 4hb_nogap_C17 & 48 & 14695.63 & 14738.0 & $0.29 \%$ & 4hb_5gap_C17 & 38 & 11581.61 & 11613.0 & $0.27 \%$ \\
\hline 4hb_nogap_C18 & 48 & 14862.74 & 14908.0 & $0.30 \%$ & 4hb_5gap_C18 & 38 & 11814.74 & 11814.0 & $-0.01 \%$ \\
\hline 4hb_nogap_C19 & 48 & 14706.54 & 14738.0 & $0.21 \%$ & 4hb_5gap_C19 & 38 & 11618.51 & 11629.0 & $0.09 \%$ \\
\hline 4hb_nogap_D01 & 36 & 11089.32 & 11088.0 & $-0.01 \%$ & 4hb_5gap_D01 & 31 & 9574.33 & 9579.0 & $0.05 \%$ \\
\hline 4hb_nogap_D02 & 48 & 14842.75 & 14895.0 & $0.35 \%$ & 4hb_5gap_D02 & 38 & 11830.8 & 11828.0 & $-0.02 \%$ \\
\hline 4hb_nogap_D03 & 48 & 14900.8 & 14952.0 & $0.34 \%$ & 4hb_5gap_D03 & 38 & 11747.73 & 11756.0 & $0.07 \%$ \\
\hline 4hb_nogap_D04 & 48 & 14767.66 & 14801.0 & $0.23 \%$ & 4hb_5gap_D04 & 38 & 11669.64 & 11678.0 & $0.07 \%$ \\
\hline 4hb_nogap_D05 & 48 & 14902.76 & 14950.0 & $0.32 \%$ & 4hb_5gap_D05 & 38 & 11827.79 & 11857.0 & $0.25 \%$ \\
\hline 4hb_nogap_D06 & 48 & 14833.75 & 14886.0 & $0.35 \%$ & 4hb_5gap_D06 & 38 & 11778.76 & 11804.0 & $0.21 \%$ \\
\hline 4hb_nogap_D07 & 48 & 14650.57 & 14686.0 & $0.24 \%$ & 4hb_5gap_D07 & 38 & 11592.58 & 11594.0 & $0.01 \%$ \\
\hline 4hb_nogap_D08 & 48 & 14682.58 & 14739.0 & $0.38 \%$ & 4hb_5gap_D08 & 38 & 11602.58 & 11636.0 & $0.29 \%$ \\
\hline 4hb_nogap_D09 & 48 & 14739.52 & 14758.0 & $0.13 \%$ & 4hb_5gap_D09 & 38 & 11744.61 & 11764.0 & $0.17 \%$ \\
\hline 4hb_nogap_D10 & 48 & 14647.63 & 14680.0 & $0.22 \%$ & 4hb_5gap_D10 & 38 & 11619.68 & 11619.0 & $-0.01 \%$ \\
\hline 4hb_nogap_D11 & 48 & 14876.72 & 14898.0 & $0.14 \%$ & 4hb_5gap_D11 & 38 & 11747.68 & 11770.0 & $0.19 \%$ \\
\hline 4hb_nogap_D12 & 48 & 15002.81 & 15039.0 & $0.24 \%$ & 4hb_5gap_D12 & 38 & 11864.76 & 11878.0 & $0.11 \%$ \\
\hline 4hb_nogap_D13 & 48 & 14776.67 & 14808.0 & $0.21 \%$ & 4hb_5gap_D13 & 38 & 11656.64 & 11666.0 & $0.08 \%$ \\
\hline 4hb_nogap_D14 & 48 & 14670.56 & 14688.0 & $0.12 \%$ & 4hb_5gap_D14 & 38 & 11606.56 & 11629.0 & $0.19 \%$ \\
\hline 4hb_nogap_D15 & 48 & 14843.67 & 14861.0 & $0.12 \%$ & 4hb_5gap_D15 & 38 & 11763.67 & 11788.0 & $0.21 \%$ \\
\hline 4hb_nogap_D16 & 48 & 14748.65 & 14787.0 & $0.26 \%$ & 4hb_5gap_D16 & 38 & 11681.65 & 11707.0 & $0.22 \%$ \\
\hline 4hb_nogap_D17 & 48 & 14912.81 & 14958.0 & $0.30 \%$ & 4hb_5gap_D17 & 38 & 11773.72 & 11801.0 & $0.23 \%$ \\
\hline 4hb_nogap_D18 & 48 & 14718.61 & 14747.0 & $0.19 \%$ & 4hb_5gap_D18 & 38 & 11565.54 & 11594.0 & $0.25 \%$ \\
\hline 4hb_nogap_D19 & 48 & 14939.79 & 14988.0 & $0.32 \%$ & 4hb_5gap_D19 & 38 & 11810.75 & 11834.0 & $0.20 \%$ \\
\hline
\end{tabular}

Supplementary Table 4. Measurement of the molecular weight of staples using MALDI-TOF. MALDI-TOF result of representative 154 staples constituting 4HB-Ref and other 4HB-5nt variations. Experimental values indicate the molecular weight from a single peak. The typical error range of MALDI-TOF analysis from the theoretical value is known as 0.2 to $0.6 \%$. 


\subsection{DNA sequence}

\begin{tabular}{|c|c|c|}
\hline \multicolumn{3}{|c|}{ 4HB design } \\
\hline Name & $\begin{array}{l}\text { Sequence }\left(5^{\prime} \rightarrow 3^{\prime}\right) \\
\end{array}$ & Color \\
\hline 4hb_001 & ACAAACAATGAATACCGCGCCCAATAGCAAGCAAATCATCCTAATCCT & \\
\hline 4hb_002 & AGAGATAATAACGTTTGAAATACCGACCGTGTGATATCATAATTGTAC & \\
\hline 4hb_003 & CCTTATATAAAATAAATGCTGATGCAAATCCAATCGCCCTTAGAAAAT & \\
\hline 4hb_004 & ACATACATTCAATACCATATCAAAATTATTTGCACGCAGGTTTACAAA & \\
\hline 4hb_005 & CAACAGAGCCAGATTATCATCATATTCCTGATTATCTTGAGGATTAGA & \\
\hline 4hb_006 & CAAAAGAATCAAAAGAATACGTGGCACAGACAATATCTGATAGCCCTC & \\
\hline 4hb_007 & AATTAAAATCACTGGATTATTTACATTGGCAGATTCTAACATCATAGC & \\
\hline 4hb_008 & TTAAACCAGAACGGAAAGCCGGCGAACGTGGCGAGATAGGGCGCTAGA & \\
\hline 4hb_009 & GAAAAAAGCCAGCGTGAACCATCACCCAAATCAAGTAATCCCTTTGAT & \\
\hline 4hb_010 & TTTTAACGGGGTAATTGTTATCCGCTCACAATTCCAAGCCTGGGAGAC & \\
\hline 4hb_011 & GCCGAGGCTGAGCCAGTGCCAAGCTTGCATGCCTGCCTGCGCAAGCAA & \\
\hline 4hb_012 & GCATCGGAATAGGTTAATATTTTGTTAAAATTCGCACCAATAGGTGCA & \\
\hline 4hb_013 & TTCATTCAGGGACGTAAAACTAGCATGTCAATCATAACCATCAAAGAA & \\
\hline 4hb_014 & TTTTCTCATAGTTTAGATACATTTCGCAAATGGTCAGCGAGCTGAGCC & \\
\hline 4hb_015 & CCCGTTCAGCGGTGTTTTAAATATGCAACTAAAGTATTCAAAGCCAAA & \\
\hline 4hb_016 & AGAACTTTAATTTAACGCCAAAAGGAATTACGAGGCTACCAGACTGAC & \\
\hline 4hb_017 & CGTAACGCATAAACGTTAATAAAACGAACTAACGGACCTGACGAGCTC & \\
\hline 4hb_018 & AGGACGAGGGTAAGGCAAAAGAATACACTAAAACACCGAAACAACGGT & \\
\hline 4hb_019 & CCAACGGGAGGTCCCTGAACAAAGTCAGAGGGTAATACGTCAAAAGCG & \\
\hline 4hb_020 & AATCGGAATCATATAGCAATAGCTATCTTACCGAAGAGCTAATGGCTG & \\
\hline 4hb_021 & TATCATTTAATGGGAATACCCAAAAGAACTGGCATGAGGCAGAGTATA & \\
\hline 4hb_022 & AGCTACTATATGGAAACGCAAAGACACCACGGAATAACATAAATGACG & \\
\hline 4hb_023 & AACATTAGAACCCCGATTGAGGGAGGGAAGGTAAATACCTGAGCTACA & \\
\hline 4hb_024 & AAACGGAGCGGACAAAATCACCAGTAGCACCATTACTTATCTAAAACT & \\
\hline 4hb_025 & AATAGAAAGCGTGTTTGCCTTTAGCGTCAGACTGTAAAATCTAAACCA & \\
\hline 4hb_026 & CAAAGTCTGAAACGGAACCAGAGCCACCACCGGAACCCGAGTAACTTG & \\
\hline 4hb_027 & CTGCCTTGACGGCACCACCAGAGCCGCCGCCAGCATTCAGAGCGACCA & \\
\hline 4hb_028 & GAGAGCCCACTAAATGGAAAGCGCAGTCTCTGAATTCGGTCCACAGTG & \\
\hline 4hb_029 & CTCACTGTGTGACAGTGCCTTGAGTAACAGTGCCCGTGCGTATTGCGT & \\
\hline 4hb_030 & TGCGAACGACGGACTCCTCAAGAGAAGGATTAGGATCAGCCAGCCGCT & \\
\hline 4hb_031 & $\begin{array}{l}\text { GCGTTTGTAAACGTGTATCACCGTACTCAGGAGGTTGGGATAGGCCTG } \\
\end{array}$ & \\
\hline 4hb_032 & GCTGACGGTAATTAGCAAGCCCAATAGGAACCCATGCAATGCCTATGC & \\
\hline 4hb_033 & CTAATTTGACCATAGCGTAACGATCTAAAGTTTTGTTACCAAAAGCAT & \\
\hline 4hb_034 & TCCAGCTCAACAAGTGAGAATAGAAAGGAACAACTAGGATTGCAGGAT & \\
\hline 4hb_035 & CGAGCAGATACAGTATCGGTTTATCAGCTTGCTTTCAATCCCCCGCAA & \\
\hline 4hb_036 & AAATAAAAATCTCCGATATATTCGGTCGCTGAGGCTAGAACCGGAGAT & \\
\hline 4hb_037 & CATCAACGAAAGGCAACGGCTACAGAGGCTTTGAGGAGGGAACCAATT & \\
\hline 4hb_038 & AAGACCAGTTACAAATAAGAAACGATTTTTTGTTTATGAGCGCTCGTT & \\
\hline 4hb_039 & ATCGAACGGGTAACGACGACAATAAACAACATGTTCCCCTTTTTCAAG & \\
\hline 4hb_040 & CAAAGCCAACGCTTTAACAACGCCAACATGTAATTTATTAAGACAGTT & \\
\hline 4hb_041 & TACCATTTATCAATTACCTTTTTTAATGGAAACAGTAGTTTATTCTCC & \\
\hline 4hb_042 & TGATTTCGCCTGAGAGGCGAATTATTCATTTCAATTATTGACGGTTAT & \\
\hline 4hb_043 & ACATAACGTTATACAGTTGAAAGGAATTGAGGAAGGCATTAGCATGCG & \\
\hline 4hb_044 & ACATGGTGAGGCCGCTGAGAGCCAGCAGCAAATGAAGCGCGTTTACAG & \\
\hline 4hb_045 & ACGCATTACCGCGTGTTTTTATAATCAGTGAGGCCACGCCTCCCATAC & \\
\hline 4hb_046 & TAAACCGCTACATATAACGTGCTTTCCTCGTTAGAATGACAGGACTAA & \\
\hline 4hb_047 & ACGTAGTCCACTTGGCCCTGAGAGAGTTGCAGCAAGTACCGTTCGAAA & \\
\hline 4hb_048 & CGAGCAGTCGGGGCCAACGCGCGGGGAGAGGCGGTTTATAAACACGTA & \\
\hline 4hb_049 & GGTAGGGGATGTATCGGCCTCAGGAAGATCGCACTCTAGCGGGGGTTT & \\
\hline 4hb_050 & CCAATAAATGTGAACAAACGGCGGATTGACCGTAATTAGTACCGAGAT & \\
\hline 4hb_051 & TGCCGAGAGATCTAGAACCCTCATATATTTTAAATGTACCGTAATGGA & \\
\hline 4hb_052 & CAGTAGGCAAGGCAGAGCATAAAGCTAAATCGGTTGCGTCTTTCAATT & \\
\hline 4hb_053 & ATGGTCCTTTTGGACTATTATAGTCAGAAGCAAAGCAAGGAATTTTAA & \\
\hline 4hb_054 & 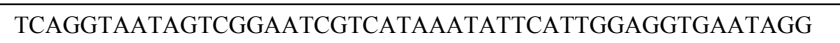 & \\
\hline 4hb_055 & GAACCATTGTGACCTTCATCAAGAGTAATCTTGACATGCAGGGATATA & \\
\hline
\end{tabular}




\begin{tabular}{|c|c|}
\hline 4hb_056 & CAAGCCCAATCCAAAATAAACAGCCATATAATATCCCAGATATATAAG \\
\hline 4hb_ 057 & CGAACTGTCCAGTTAAACCAAGAATAAACACCGGAAAATAAGGCCCAG \\
\hline 4hb_058 & AGCATCGCCATATCAACAGTAGGGCTTATTAATTTTCAAGACAAAAAT \\
\hline 4hb_059 & TTCATCATTTGAAAATCATAAAATTGCGTAGATTTTTAAAACAGACCA \\
\hline 4hb_ 060 & $\begin{array}{l}\text { ATTAAATCGCGCATTGCTTTGAATACCATAATACATAGATGATGACCG } \\
\end{array}$ \\
\hline 4hb_061 & GAAAGCAAATCATAATTTTAGTCTTTAATGCGCGAATTTTGAATAGCA \\
\hline 4hb_062 & AGCCCAGTGCCAGGTCAGTATTAACACCATTAGTAAACCAGTCAAGCG \\
\hline 4hb_063 & ACCGCCTGAGAACAGCCATTCGAAAGGAGCGGGCGCAAGGAAGGAGAG \\
\hline 4hb_064 & TTGGCGAGCACGGGGCGCGTACTATGGTATCGGCAATTTTTGGGTTCA \\
\hline 4hb_ 065 & $\begin{array}{l}\text { TTTTTCACCGCCATTAAAGAGAAGCATAAAGTGTAACACAACATAGGA } \\
\end{array}$ \\
\hline 4hb_066 & TCGGATGAATCGAAACCTGTCGTGCCAGCATTCAGGAGGTCGACATTC \\
\hline 4hb_067 & ATAAACGACAGTGCTGCAAGTCAGCTCATTTTTTAATTAAATTTGAGA \\
\hline 4hb_ 068 & CTCATCCGTGGGAGCGAGTAACAACCCGGTCAAATCTGTACCCCACCC \\
\hline 4hb_069 & ACAAAAAATTTTTACAAAGGTATTTTCATTTGGGGCATAACCTGGCCT \\
\hline 4hb_070 & $\begin{array}{l}\text { TTCTTAAAGCCTCAAAGAATTAGCAAAAATTCGAGCCGGTGTCTTTTT } \\
\end{array}$ \\
\hline 4hb_071 & GTTGTTTACCCTATAAGAGGTATCATAACCCTCGTTATAGTAAGCAAA \\
\hline 4hb_072 & CGATCAATACTGAAAATGTTTAGACTGGAGGCTTGCACAACATTCGAC \\
\hline 4hb_ 073 & GATCCTGGCTGAATTACCTTGCGATTATACCAAGCGTCATCTTTCAGC \\
\hline 4hb_nogap_A01 & TCTTTCCAGAGCCTAATTTGACGCGAGGAATATCAGAGAGATAACCCA \\
\hline 4hb_nogap_A02 & TCTTTCCTTATCATTCCAAGAGAACAAGAAGAAAAGTAAGCAGATAGC \\
\hline 4hb_nogap_A03 & CAAATTCTTACCAGTATAAATATATTTTTCCTTATTACGCAGTATGTT \\
\hline 4hb_nogap_A04 & CTGAGAAGAGTCAATAGTGATTTTTAACTTGTCACAATCAATAGAAAA \\
\hline 4hb_nogap_A05 & TCGGGAGAAACAATAACGGATGTTTGGAAAATTATTCATTAAAGGTGA \\
\hline 4hb_nogap_A06 & CGTATTAAATCCTTTGCCCGTATCATTTAGGCCGGAAACGTCACCAAT \\
\hline 4hb_nogap_A07 & CCAGCAGAAGATAAAACAGATCTGGCCATCATCGGCATTTTCGGTCAT \\
\hline 4hb_nogap_A08 & CTGGTAATATCCAGAACAATTCATGGAATCAGAGCCGCCACCCTCAGA \\
\hline 4hb_nogap_A09 & CACCCGCCGCGCTTAATGCGTCGGAACCGGTTGAGGCAGGTCAGACGA \\
\hline 4hb_nogap_A10 & TTGTTCCAGTTTGGAACAAGCAAAGGGCCAGTAAGCGTCATACATGGC \\
\hline 4hb_nogap_A11 & TGCGCTCACTGCCCGCTTTCCTCGAATTGTTAATGCCCCCTGCCTATT \\
\hline 4hb_nogap_A12 & ATTACGCCAGCTGGCGAAAGACGCCAGGTTTTGCTCAGTACCAGGCGG \\
\hline 4hb_nogap_A13 & TAGCCAGCTTTCATCAACATAAACAGGACCACCCTCAGAACCGCCACC \\
\hline 4hb_nogap_A14 & CGGAGAGGGTAGCTATTTTTTGAGAGTCCACTGAGTTTCGTCACCAGT \\
\hline 4hb_nogap_A15 & TAACATCCAATAAATCATACTGATTCCCCAGACGTTAGTAAATGAATT \\
\hline 4hb_nogap_A16 & TAGAGAGTACCTTTAATTGCCTTAGAGCGCGAATAATAATTTTTTCAC \\
\hline 4hb_nogap_A17 & AAGAAGTTTTGCCAGAGGGGTTGAGATTTTTCTTAAACAGCTTGATAC \\
\hline 4hb_nogap_A18 & GGTTTAATTTCAACTTTAATTGGCTCATGTTAAAGGCCGCTTTTGCGG \\
\hline 4hb_nogap_A19 & GTGTCGAAATCCGCGACCTGTACGTAATCTTTTTCA \\
\hline 4hb_nogap_B01 & TTAGCGAACCTCCCGACTTGCGCTAACGAATGAAAA \\
\hline 4hb_nogap_B02 & CCGTTTTTATTTTCATCGTAAATAATCGCAGAACGCGCCTGTTTATCA \\
\hline 4hb_nogap_B03 & AATTTCATCTTCTGACCTAAATATGCGTGCATTTTCGAGCCAGTAATA \\
\hline 4hb_nogap_B04 & GGCTTAGGTTGGGTTATATATAGATTAACAATATATGTGAGTGAATAA \\
\hline 4hb_nogap_B05 & ACTTCTGAATAATGGAAGGGGTACCTTTAAAAGAAGATGATGAAACAA \\
\hline 4hb_nogap_B06 & $\begin{array}{l}\text { GAACAAAGAAACCACCAGAAAATTCGACAATATCTTTAGGAGCACTAA } \\
\end{array}$ \\
\hline 4hb_nogap_B07 & AGATAGAACCCTTCTGACCTCCGAACGAAGCATCACCTTGCTGAACCT \\
\hline 4hb_nogap_B08 & CTACATTTTGACGCTCAATCCTATCGGCAAGAGTCTGTCCATCACGCA \\
\hline 4hb_nogap_B09 & AGGGAGCCCCCGATTTAGAGGCGTAACCGGAGCTAAACAGGAGGCCGA \\
\hline 4hb_nogap_B10 & AACCGTCTATCAGGGCGATGTAGGGTTGGCTGGTTTGCCCCAGCAGGC \\
\hline 4hb_nogap_B11 & ATCATGGTCATAGCTGTTTCCATTAATTGGGCGCCAGGGTGGTTTTTC \\
\hline 4hb_nogap_B12 & TCCCAGTCACGACGTTGTAAGGCCTCTTTTTCCGGCACCGCTTCTGGT \\
\hline 4hb_nogap_B13 & TGTATAAGCAAATATTTAAACTGGCCTTTCACGTTGGTGTAGATGGGC \\
\hline 4hb_nogap_B14 & GCAAACAAGAGAATCGATGAATAAATTAGAGTAATGTGTAGGTAAAGA \\
\hline 4hb_nogap_B15 & CTGCGAACGAGTAGATTTAGTAGTAGTAACATTATGACCCTGTAATAC \\
\hline 4hb_nogap_B16 & $\begin{array}{l}\text { TTGCTGAATATAATGCTGTAACAGGTCATCAAAAAGATTAAGAGGAAG } \\
\end{array}$ \\
\hline 4hb_nogap_B17 & AATACCACATTCAACTAATGAGGCTTTTTCAAATGCTTTAAACAGTTC \\
\hline 4hb_nogap_B18 & CCAGTCAGGACGTTGGGAAGTGGGCTTGATATTCATTACCCAAATCAA \\
\hline 4hb_nogap_B19 & CAACCTAAGCCTGATAGAACTGACCAACTTTGAAAG \\
\hline 4hb_nogap_B20 & CAGACGGTCAATCATAACTAAAGAGCCACTACGAAGGCAC \\
\hline 4hb_nogap_C01 & TAGCAGCCTTTACAGAACTGAACATTTGAAGCCTTAAATC \\
\hline 4hb_nogap_C02 & GAACAAGAAAAATTATTTATAATTGAGTGAAGGCTTATCCGGTATTCT \\
\hline
\end{tabular}




\begin{tabular}{|c|c|}
\hline 4hb_nogap_C03 & CGACAAAAGGTAAAGTAATTCAAAGTTAGTTAAATAAGTACCGCACTC \\
\hline 4hb_nogap_C04 & CGTCGCTATTAAATTGAGAAAACGTAGAAGAACGCGAGAAAACTTTTT \\
\hline 4hb_nogap_C05 & ATTAATTACATTTAACAATTTATGGTTTAAATAAAGGGTCTGAGAGAC \\
\hline 4hb_nogap_C06 & GCCGTCAATAGAAGTTACAATCACCGTCGCAATTCATCAATATAATCC \\
\hline 4hb_nogap_C07 & AATCAATATCTGGTCAGTTGCCATCGATGGCTATTAAAAGTTTGAGTA \\
\hline 4hb_nogap_C08 & AATACTTCTTTGGCCTGCAACCCTTATTCACGACCAGTAATAAAAGGG \\
\hline 4hb_nogap_C09 & CAGGAACGGTACGCCAGAATCCACCCTCGAAGAAAGGCAACAGGAAAA \\
\hline 4hb_nogap_C10 & GGTGGTTCCGAATGCTTTGACCTTGATAGTCGAGGTGCCGTAAAGCAC \\
\hline 4hb_nogap_C11 & GGGCAACAGCTGATTGCCCTGATGATACACGAGCCGACGTGGACTCCA \\
\hline 4hb_nogap_C12 & AGCGCCATTCGCCTGCATTAAACCTATTTCTAGAGGATCCCCGGGTAC \\
\hline 4hb_nogap_C13 & TCTGCCAGTTTGAGGGGACGGTGCCGTCTTGTTAAAGCGATTAAGTTG \\
\hline 4hb_nogap_C14 & AGGCCGGAGACATCGGATTCGAACCGCCGGTTGATAATCAGAAAAGCC \\
\hline 4hb_nogap_C15 & TTTATTTCAACGCAAGGATAACTACAACTTTAGCTACTATCAGGTCAT \\
\hline 4hb_nogap_C16 & TATCGCGTTTTATTAAGCAAGTATGGGAGGAAGTTTCATTCCATATAA \\
\hline 4hb_nogap_C17 & CATAAATCAAAAATCAGGTCAAAATCTCAGCAACACTCATTTTTGCGG \\
\hline 4hb_nogap_C18 & ATTCAGTGAATAATAGCGTCAGTTGCGCATTACAGGTAGAAAGATTCA \\
\hline 4hb_nogap_C19 & GTACAGACCAGGCGCATAGGGTCACCCTGACCCCCAATGCGATTTTAA \\
\hline 4hb_nogap_D01 & GAGAATTAGAGAATAACAATTTTATCCTGAATCTTA \\
\hline 4hb_nogap_D02 & CCCAATAATAAGAGCAAGAATAGATAAGTTTACGAGCATGTAGAAACC \\
\hline 4hb_nogap_D03 & AAGGAAACCGAGGAAACGCAAATATAAAACTAGAAAAAGCCTGTTTAG \\
\hline 4hb_nogap_D04 & ACATACATAAAGGTGGCAACGCTTCTGTATCCTTGAAAACATAGCGAT \\
\hline 4hb_nogap_D05 & GCGCCAAAGACAAAAGGGCGCAAGAAAAACGTCAGATGAATATACAGT \\
\hline 4hb_nogap_D06 & ACTTGAGCCATTTGGGAATTTAATAGATTTAGAAGTATTAGACTTTAC \\
\hline 4hb_nogap_D07 & GCACCGTAATCAGTAGCGACTATCAAACCCTAAAACATCGCCATTAAA \\
\hline 4hb_nogap_D08 & TTTGCCATCTTTTCATAATCAACCGTTGCTTGCCTGAGTAGAAGAACT \\
\hline 4hb_nogap_D09 & CCACCACCCTCAGAGCCGCCAGGGATTTTGGCAAGTGTAGCGGTCACG \\
\hline 4hb_nogap_D10 & CAAACAAATAAATCCTCATTATCCTGTTATAAATCAAAAGAATAGCCC \\
\hline 4hb_nogap_D11 & GTGTACTGGTAATAAGTTTTCACCAGTGGTGCCTAATGAGTGAGCTAA \\
\hline 4hb_nogap_D12 & TGAAACATGAAAGTATTAAGGAAACCAGCTGTTGGGAAGGGCGATCGG \\
\hline 4hb_nogap_D13 & GGGTTGATATAAGTATAGCCCGTAACCGAACGCCATCAAAAATAATTC \\
\hline 4hb_nogap_D14 & TCAGAGCCACCACCCTCATTAAAGGGTGTATGATATTCAACCGTTCTA \\
\hline 4hb_nogap_D15 & GTAGCATTCCACAGACAGCCGCGGGAGAAAAAGGTGGCATCAATTCTA \\
\hline 4hb_nogap_D16 & GCTAAACAACTTTCAACAGTAAAGACTTGAACCAGACCGGAAGCAAAC \\
\hline 4hb_nogap_D17 & AAAAAGGCTCCAAAAGGAGCAACGAGAAGACGATAAAAACCAAAATAG \\
\hline 4hb_nogap_D18 & AATGACAACAACCATCGCCCACAAAGCTGAAACACCAGAACGAGTAGT \\
\hline 4hb_nogap_D19 & AGCGAAAGACAGCATCGGAACAGATGAAAGTACAACGGAGATTTGTAT \\
\hline 4hb_1gap_A01 & CTTTCCAGAGCCTAATTTGACGCGAGGAATATCAGAGAGATAACCC \\
\hline 4hb_1gap_A02 & СТTTCCTTATCATTCCAAGAGAACAAGAAGAAAAGTAAGCAGATAG \\
\hline 4hb_1gap_A03 & AAATTCTTACCAGTATAAATATATTTTTCCTTATTACGCAGTATGT \\
\hline 4hb_1gap_A04 & TGAGAAGAGTCAATAGTGATTTTTAACTTGTCACAATCAATAGAAA \\
\hline 4hb_1gap_A05 & CGGGAGAAACAATAACGGATGTTTGGAAAATTATTCATTAAAGGTG \\
\hline 4hb_1gap_A06 & GTATTAAATCCTTTGCCCGTATCATTTAGGCCGGAAACGTCACCAA \\
\hline 4hb_1gap_A07 & CAGCAGAAGATAAAACAGATCTGGCCATCATCGGCATTTTCGGTCA \\
\hline 4hb_1gap_A08 & TGGTAATATCCAGAACAATTCATGGAATCAGAGCCGCCACCCTCAG \\
\hline 4hb_1gap_A09 & ACCCGCCGCGCTTAATGCGTCGGAACCGGTTGAGGCAGGTCAGACG \\
\hline 4hb_1gap_A10 & TGTTCCAGTTTGGAACAAGCAAAGGGCCAGTAAGCGTCATACATGG \\
\hline 4hb_1gap_A11 & GCGCTCACTGCCCGCTTTCCTCGAATTGTTAATGCCCCCTGCCTAT \\
\hline 4hb_1gap_A12 & TTACGCCAGCTGGCGAAAGACGCCAGGTTTTGCTCAGTACCAGGCG \\
\hline 4hb_1gap_A13 & AGCCAGCTTTCATCAACATAAACAGGACCACCCTCAGAACCGCCAC \\
\hline 4hb_1gap_A14 & GGAGAGGGTAGCTATTTTTTGAGAGTCCACTGAGTTTCGTCACCAG \\
\hline 4hb_1gap_A15 & AACATCCAATAAATCATACTGATTCCCCAGACGTTAGTAAATGAAT \\
\hline 4hb_1gap_A16 & AGAGAGTACCTTTAATTGCCTTAGAGCGCGAATAATAATTTTTTCA \\
\hline 4hb_1gap_A17 & AGAAGTTTTGCCAGAGGGGTTGAGATTTTTCTTAAACAGCTTGATA \\
\hline 4hb_1gap_A18 & GTTTAATTTCAACTTTAATTGGCTCATGTTAAAGGCCGCTTTTGCG \\
\hline 4hb_1gap_A19 & TGTCGAAATCCGCGACCTGTACGTAATCTTTTTCA \\
\hline 4hb_1gap_B01 & TAGCGAACCTCCCGACTTGCGCTAACGAATGAAAA \\
\hline 4hb_1gap_B02 & CGTTTTTATTTTCATCGTAAATAATCGCAGAACGCGCCTGTTTATC \\
\hline 4hb_1gap_B03 & ATTTCATCTTCTGACCTAAATATGCGTGCATTTTCGAGCCAGTAAT \\
\hline 4hb_1gap_B04 & GCTTAGGTTGGGTTATATATAGATTAACAATATATGTGAGTGAATA \\
\hline
\end{tabular}




\begin{tabular}{|c|c|}
\hline 4hb_1gap_B05 & СTTCTGAATAATGGAAGGGGTACCTTTAAAAGAAGATGATGAAACA \\
\hline 4hb_1gap_B06 & AACAAAGAAACCACCAGAAAATTCGACAATATCTTTAGGAGCACTA \\
\hline 4hb_1gap_B07 & GATAGAACCCTTCTGACCTCCGAACGAAGCATCACCTTGCTGAACC \\
\hline 4hb_1gap_B08 & TACATTTTGACGCTCAATCCTATCGGCAAGAGTCTGTCCATCACGC \\
\hline 4hb_1gap_B09 & GGGAGCCCCCGATTTAGAGGCGTAACCGGAGCTAAACAGGAGGCCG \\
\hline 4hb_1gap_B10 & ACCGTCTATCAGGGCGATGTAGGGTTGGCTGGTTTGCCCCAGCAGG \\
\hline 4hb_1gap_B11 & $\begin{array}{l}\text { TCATGGTCATAGCTGTTTCCATTAATTGGGCGCCAGGGTGGTTTTT } \\
\end{array}$ \\
\hline 4hb_1gap_B12 & CCCAGTCACGACGTTGTAAGGCCTCTTTTTCCGGCACCGCTTCTGG \\
\hline 4hb_1gap_B13 & GTATAAGCAAATATTTAAACTGGCCTTTCACGTTGGTGTAGATGGG \\
\hline 4hb_1gap_B14 & CAAACAAGAGAATCGATGAATAAATTAGAGTAATGTGTAGGTAAAG \\
\hline 4hb_1gap_B15 & TGCGAACGAGTAGATTTAGTAGTAGTAACATTATGACCCTGTAATA \\
\hline 4hb_1gap_B16 & TGCTGAATATAATGCTGTAACAGGTCATCAAAAAGATTAAGAGGAA \\
\hline 4hb_1gap_B17 & ATACCACATTCAACTAATGAGGCTTTTTCAAATGCTTTAAACAGTT \\
\hline 4hb_1gap_B18 & CAGTCAGGACGTTGGGAAGTGGGCTTGATATTCATTACCCAAATCA \\
\hline 4hb_1gap_B19 & CAACCTAAGCCTGATAGAACTGACCAACTTTGAAA \\
\hline 4hb_1gap_B20 & CAGACGGTCAATCATAACTAAAGAGCCACTACGAAGGCA \\
\hline 4hb_1gap_C01 & AGCAGCCTTTACAGAACTGAACATTTGAAGCCTTAAATC \\
\hline 4hb_1gap_C02 & AACAAGAAAAATTATTTATAATTGAGTGAAGGCTTATCCGGTATTC \\
\hline 4hb_1gap_C03 & GACAAAAGGTAAAGTAATTCAAAGTTAGTTAAATAAGTACCGCACT \\
\hline 4hb_1gap_C04 & GTCGCTATTAAATTGAGAAAACGTAGAAGAACGCGAGAAAACTTTT \\
\hline 4hb_1gap_C05 & TTAATTACATTTAACAATTTATGGTTTAAATAAAGGGTCTGAGAGA \\
\hline 4hb_1gap_C06 & CCGTCAATAGAAGTTACAATCACCGTCGCAATTCATCAATATAATC \\
\hline 4hb_1gap_C07 & ATCAATATCTGGTCAGTTGCCATCGATGGCTATTAAAAGTTTGAGT \\
\hline 4hb_1gap_C08 & ATACTTCTTTGGCCTGCAACCCTTATTCACGACCAGTAATAAAAGG \\
\hline 4hb_1gap_C09 & AGGAACGGTACGCCAGAATCCACCCTCGAAGAAAGGCAACAGGAAA \\
\hline 4hb_1gap_C10 & $\begin{array}{l}\text { GTGGTTCCGAATGCTTTGACCTTGATAGTCGAGGTGCCGTAAAGCA } \\
\end{array}$ \\
\hline 4hb_1gap_C11 & GGCAACAGCTGATTGCCCTGATGATACACGAGCCGACGTGGACTCC \\
\hline 4hb_1gap_C12 & GCGCCATTCGCCTGCATTAAACCTATTTCTAGAGGATCCCCGGGTA \\
\hline 4hb_1gap_C13 & CTGCCAGTTTGAGGGGACGGTGCCGTCTTGTTAAAGCGATTAAGTT \\
\hline 4hb_1gap_C14 & GGCCGGAGACATCGGATTCGAACCGCCGGTTGATAATCAGAAAAGC \\
\hline 4hb_1gap_C15 & TTATTTCAACGCAAGGATAACTACAACTTTAGCTACTATCAGGTCA \\
\hline 4hb_1gap_C16 & ATCGCGTTTTATTAAGCAAGTATGGGAGGAAGTTTCATTCCATATA \\
\hline 4hb_1gap_C17 & ATAAATCAAAAATCAGGTCAAAATCTCAGCAACACTCATTTTTGCG \\
\hline 4hb_1gap_C18 & TTCAGTGAATAATAGCGTCAGTTGCGCATTACAGGTAGAAAGATTC \\
\hline 4hb_1gap_C19 & TACAGACCAGGCGCATAGGGTCACCCTGACCCCCAATGCGATTTTA \\
\hline 4hb_1gap_D01 & GAGAATTAGAGAATAACAATTTTATCCTGAATCTT \\
\hline 4hb_1gap_D02 & CCAATAATAAGAGCAAGAATAGATAAGTTTACGAGCATGTAGAAAC \\
\hline 4hb_1gap_D03 & AGGAAACCGAGGAAACGCAAATATAAAACTAGAAAAAGCCTGTTTA \\
\hline 4hb_1gap_D04 & CATACATAAAGGTGGCAACGCTTCTGTATCCTTGAAAACATAGCGA \\
\hline 4hb_1gap_D05 & CGCCAAAGACAAAAGGGCGCAAGAAAAACGTCAGATGAATATACAG \\
\hline 4hb_1gap_D06 & CTTGAGCCATTTGGGAATTTAATAGATTTAGAAGTATTAGACTTTA \\
\hline 4hb_1gap_D07 & CACCGTAATCAGTAGCGACTATCAAACCCTAAAACATCGCCATTAA \\
\hline 4hb_1gap_D08 & TTGCCATCTTTTCATAATCAACCGTTGCTTGCCTGAGTAGAAGAAC \\
\hline 4hb_1gap_D09 & CACCACCCTCAGAGCCGCCAGGGATTTTGGCAAGTGTAGCGGTCAC \\
\hline 4hb_1gap_D10 & AAACAAATAAATCCTCATTATCCTGTTATAAATCAAAAGAATAGCC \\
\hline 4hb_1gap_D11 & TGTACTGGTAATAAGTTTTCACCAGTGGTGCCTAATGAGTGAGCTA \\
\hline 4hb_1gap_D12 & GAAACATGAAAGTATTAAGGAAACCAGCTGTTGGGAAGGGCGATCG \\
\hline 4hb_1gap_D13 & GGTTGATATAAGTATAGCCCGTAACCGAACGCCATCAAAAATAATT \\
\hline 4hb_1gap_D14 & CAGAGCCACCACCCTCATTAAAGGGTGTATGATATTCAACCGTTCT \\
\hline 4hb_1gap_D15 & TAGCATTCCACAGACAGCCGCGGGAGAAAAAGGTGGCATCAATTCT \\
\hline 4hb_1gap_D16 & СТAAACAACTTTCAACAGTAAAGACTTGAACCAGACCGGAAGCAAA \\
\hline 4hb_1gap_D17 & AAAAGGCTCCAAAAGGAGCAACGAGAAGACGATAAAAACCAAAATA \\
\hline 4hb_1gap_D18 & ATGACAACAACCATCGCCCACAAAGCTGAAACACCAGAACGAGTAG \\
\hline 4hb_1gap_D19 & $\begin{array}{l}\text { GCGAAAGACAGCATCGGAACAGATGAAAGTACAACGGAGATTTGTA } \\
\end{array}$ \\
\hline 4hb_3gap_A01 & TTCCAGAGCCTAATTTGACGCGAGGAATATCAGAGAGATAAC \\
\hline 4hb_3gap_A02 & TTCCTTATCATTCCAAGAGAACAAGAAGAAAAGTAAGCAGAT \\
\hline 4hb_3gap_A03 & ATTCTTACCAGTATAAATATATTTTTCCTTATTACGCAGTAT \\
\hline 4hb_3gap_A04 & AGAAGAGTCAATAGTGATTTTTAACTTGTCACAATCAATAGA \\
\hline 4hb_3gap_A05 & GGAGAAACAATAACGGATGTTTGGAAAATTATTCATTAAAGG \\
\hline
\end{tabular}




\begin{tabular}{|c|c|}
\hline 4hb_3gap_A06 & ATTAAATCCTTTGCCCGTATCATTTAGGCCGGAAACGTCACC \\
\hline 4hb_3gap_A07 & GCAGAAGATAAAACAGATCTGGCCATCATCGGCATTTTCGGT \\
\hline 4hb_3gap_A08 & GTAATATCCAGAACAATTCATGGAATCAGAGCCGCCACCCTC \\
\hline 4hb_3gap_A09 & CCGCCGCGCTTAATGCGTCGGAACCGGTTGAGGCAGGTCAGA \\
\hline 4hb_3gap_A10 & TTCCAGTTTGGAACAAGCAAAGGGCCAGTAAGCGTCATACAT \\
\hline 4hb_3gap_A11 & GCTCACTGCCCGCTTTCCTCGAATTGTTAATGCCCCCTGCCT \\
\hline 4hb_3gap_A12 & $\begin{array}{l}\text { ACGCCAGCTGGCGAAAGACGCCAGGTTTTGCTCAGTACCAGG } \\
\end{array}$ \\
\hline 4hb_3gap_A13 & CCAGCTTTCATCAACATAAACAGGACCACCCTCAGAACCGCC \\
\hline 4hb_3gap_A14 & AGAGGGTAGCTATTTTTTGAGAGTCCACTGAGTTTCGTCACC \\
\hline 4hb_3gap_A15 & CATCCAATAAATCATACTGATTCCCCAGACGTTAGTAAATGA \\
\hline 4hb_3gap_A16 & AGAGTACCTTTAATTGCCTTAGAGCGCGAATAATAATTTTTT \\
\hline 4hb_3gap_A17 & AAGTTTTGCCAGAGGGGTTGAGATTTTTCTTAAACAGCTTGA \\
\hline 4hb_3gap_A18 & TTAATTTCAACTTTAATTGGCTCATGTTAAAGGCCGCTTTTG \\
\hline 4hb_3gap_A19 & TCGAAATCCGCGACCTGTACGTAATCTTTTTCA \\
\hline 4hb_3gap_B01 & GCGAACCTCCCGACTTGCGCTAACGAATGAAAA \\
\hline 4hb_3gap_B02 & TTTTTATTTTCATCGTAAATAATCGCAGAACGCGCCTGTTTA \\
\hline 4hb_3gap_B03 & TTCATCTTCTGACCTAAATATGCGTGCATTTTCGAGCCAGTA \\
\hline 4hb_3gap_B04 & TTAGGTTGGGTTATATATAGATTAACAATATATGTGAGTGAA \\
\hline 4hb_3gap_B05 & TCTGAATAATGGAAGGGGTACCTTTAAAAGAAGATGATGAAA \\
\hline 4hb_3gap_B06 & CAAAGAAACCACCAGAAAATTCGACAATATCTTTAGGAGCAC \\
\hline 4hb_3gap_B07 & TAGAACCCTTCTGACCTCCGAACGAAGCATCACCTTGCTGAA \\
\hline 4hb_3gap_B08 & CATTTTGACGCTCAATCCTATCGGCAAGAGTCTGTCCATCAC \\
\hline 4hb_3gap_B09 & GAGCCCCCGATTTAGAGGCGTAACCGGAGCTAAACAGGAGGC \\
\hline 4hb_3gap_B10 & CGTCTATCAGGGCGATGTAGGGTTGGCTGGTTTGCCCCAGCA \\
\hline 4hb_3gap_B11 & ATGGTCATAGCTGTTTCCATTAATTGGGCGCCAGGGTGGTTT \\
\hline 4hb_3gap_B12 & $\begin{array}{l}\text { CAGTCACGACGTTGTAAGGCCTCTTTTTCCGGCACCGCTTCT } \\
\end{array}$ \\
\hline 4hb_3gap_B13 & ATAAGCAAATATTTAAACTGGCCTTTCACGTTGGTGTAGATG \\
\hline 4hb_3gap_B14 & AACAAGAGAATCGATGAATAAATTAGAGTAATGTGTAGGTAA \\
\hline 4hb_3gap_B15 & CGAACGAGTAGATTTAGTAGTAGTAACATTATGACCCTGTAA \\
\hline 4hb_3gap_B16 & CTGAATATAATGCTGTAACAGGTCATCAAAAAGATTAAGAGG \\
\hline 4hb_3gap_B17 & ACCACATTCAACTAATGAGGCTTTTTCAAATGCTTTAAACAG \\
\hline 4hb_3gap_B18 & GTCAGGACGTTGGGAAGTGGGCTTGATATTCATTACCCAAAT \\
\hline 4hb_3gap_B19 & CAACCTAAGCCTGATAGAACTGACCAACTTTGA \\
\hline 4hb_3gap_B20 & CAGACGGTCAATCATAACTAAAGAGCCACTACGAAGG \\
\hline 4hb_3gap_C01 & CAGCCTTTACAGAACTGAACATTTGAAGCCTTAAATC \\
\hline 4hb_3gap_C02 & CAAGAAAAATTATTTATAATTGAGTGAAGGCTTATCCGGTAT \\
\hline 4hb_3gap_C03 & CAAAAGGTAAAGTAATTCAAAGTTAGTTAAATAAGTACCGCA \\
\hline 4hb_3gap_C04 & CGCTATTAAATTGAGAAAACGTAGAAGAACGCGAGAAAACTT \\
\hline 4hb_3gap_C05 & AATTACATTTAACAATTTATGGTTTAAATAAAGGGTCTGAGA \\
\hline 4hb_3gap_C06 & GTCAATAGAAGTTACAATCACCGTCGCAATTCATCAATATAA \\
\hline 4hb_3gap_C07 & CAATATCTGGTCAGTTGCCATCGATGGCTATTAAAAGTTTGA \\
\hline 4hb_3gap_C08 & ACTTCTTTGGCCTGCAACCCTTATTCACGACCAGTAATAAAA \\
\hline 4hb_3gap_C09 & GAACGGTACGCCAGAATCCACCCTCGAAGAAAGGCAACAGGA \\
\hline 4hb_3gap_C10 & GGTTCCGAATGCTTTGACCTTGATAGTCGAGGTGCCGTAAAG \\
\hline 4hb_3gap_C11 & CAACAGCTGATTGCCCTGATGATACACGAGCCGACGTGGACT \\
\hline 4hb_3gap_C12 & GCCATTCGCCTGCATTAAACCTATTTCTAGAGGATCCCCGGG \\
\hline 4hb_3gap_C13 & GCCAGTTTGAGGGGACGGTGCCGTCTTGTTAAAGCGATTAAG \\
\hline 4hb_3gap_C14 & CCGGAGACATCGGATTCGAACCGCCGGTTGATAATCAGAAAA \\
\hline 4hb_3gap_C15 & ATTTCAACGCAAGGATAACTACAACTTTAGCTACTATCAGGT \\
\hline 4hb_3gap_C16 & CGCGTTTTATTAAGCAAGTATGGGAGGAAGTTTCATTCCATA \\
\hline 4hb_3gap_C17 & AAATCAAAAATCAGGTCAAAATCTCAGCAACACTCATTTTTG \\
\hline 4hb_3gap_C18 & CAGTGAATAATAGCGTCAGTTGCGCATTACAGGTAGAAAGAT \\
\hline 4hb_3gap_C19 & CAGACCAGGCGCATAGGGTCACCCTGACCCCCAATGCGATTT \\
\hline 4hb_3gap_D01 & GAGAATTAGAGAATAACAATTTTATCCTGAATC \\
\hline 4hb_3gap_D02 & AATAATAAGAGCAAGAATAGATAAGTTTACGAGCATGTAGAA \\
\hline 4hb_3gap_D03 & GAAACCGAGGAAACGCAAATATAAAACTAGAAAAAGCCTGTT \\
\hline 4hb_3gap_D04 & TACATAAAGGTGGCAACGCTTCTGTATCCTTGAAAACATAGC \\
\hline 4hb_3gap_D05 & CCAAAGACAAAAGGGCGCAAGAAAAACGTCAGATGAATATAC \\
\hline 4hb_3gap_D06 & TGAGCCATTTGGGAATTTAATAGATTTAGAAGTATTAGACTT \\
\hline
\end{tabular}




\begin{tabular}{|c|c|}
\hline 4hb_3gap_D07 & CCGTAATCAGTAGCGACTATCAAACCCTAAAACATCGCCATT \\
\hline 4hb_3gap_D08 & GCCATCTTTTCATAATCAACCGTTGCTTGCCTGAGTAGAAGA \\
\hline 4hb_3gap_D09 & CCACCCTCAGAGCCGCCAGGGATTTTGGCAAGTGTAGCGGTC \\
\hline 4hb_3gap_D10 & ACAAATAAATCCTCATTATCCTGTTATAAATCAAAAGAATAG \\
\hline 4hb_3gap_D11 & TACTGGTAATAAGTTTTCACCAGTGGTGCCTAATGAGTGAGC \\
\hline 4hb_3gap_D12 & AACATGAAAGTATTAAGGAAACCAGCTGTTGGGAAGGGCGAT \\
\hline 4hb_3gap_D13 & $\begin{array}{l}\text { TTGATATAAGTATAGCCCGTAACCGAACGCCATCAAAAATAA } \\
\end{array}$ \\
\hline 4hb_3gap_D14 & GAGCCACCACCCTCATTAAAGGGTGTATGATATTCAACCGTT \\
\hline 4hb_3gap_D15 & GCATTCCACAGACAGCCGCGGGAGAAAAAGGTGGCATCAATT \\
\hline 4hb_3gap_D16 & AAACAACTTTCAACAGTAAAGACTTGAACCAGACCGGAAGCA \\
\hline 4hb_3gap_D17 & AAGGCTCCAAAAGGAGCAACGAGAAGACGATAAAAACCAAAA \\
\hline 4hb_3gap_D18 & GACAACAACCATCGCCCACAAAGCTGAAACACCAGAACGAGT \\
\hline 4hb_3gap_D19 & GAAAGACAGCATCGGAACAGATGAAAGTACAACGGAGATTTG \\
\hline 4hb_5gap_A01 & CCAGAGCCTAATTTGACGCGAGGAATATCAGAGAGATA \\
\hline 4hb_5gap_A02 & CCTTATCATTCCAAGAGAACAAGAAGAAAAGTAAGCAG \\
\hline 4hb_5gap_A03 & TCTTACCAGTATAAATATATTTTTCCTTATTACGCAGT \\
\hline 4hb_5gap_A04 & AAGAGTCAATAGTGATTTTTAACTTGTCACAATCAATA \\
\hline 4hb_5gap_A05 & AGAAACAATAACGGATGTTTGGAAAATTATTCATTAAA \\
\hline 4hb_5gap_A06 & TAAATCCTTTGCCCGTATCATTTAGGCCGGAAACGTCA \\
\hline 4hb_5gap_A07 & AGAAGATAAAACAGATCTGGCCATCATCGGCATTTTCG \\
\hline 4hb_5gap_A08 & AATATCCAGAACAATTCATGGAATCAGAGCCGCCACCC \\
\hline 4hb_5gap_A09 & GCCGCGCTTAATGCGTCGGAACCGGTTGAGGCAGGTCA \\
\hline 4hb_5gap_A10 & CCAGTTTGGAACAAGCAAAGGGCCAGTAAGCGTCATAC \\
\hline 4hb_5gap_A11 & TCACTGCCCGCTTTCCTCGAATTGTTAATGCCCCCTGC \\
\hline 4hb_5gap_A12 & GCCAGCTGGCGAAAGACGCCAGGTTTTGCTCAGTACCA \\
\hline 4hb_5gap_A13 & AGCTTTCATCAACATAAACAGGACCACCCTCAGAACCG \\
\hline 4hb_5gap_A14 & AGGGTAGCTATTTTTTGAGAGTCCACTGAGTTTCGTCA \\
\hline 4hb_5gap_A15 & TCCAATAAATCATACTGATTCCCCAGACGTTAGTAAAT \\
\hline 4hb_5gap_A16 & AGTACCTTTAATTGCCTTAGAGCGCGAATAATAATTTT \\
\hline 4hb_5gap_A17 & GTTTTGCCAGAGGGGTTGAGATTTTTCTTAAACAGCTT \\
\hline 4hb_5gap_A18 & AATTTCAACTTTAATTGGCTCATGTTAAAGGCCGCTTT \\
\hline 4hb_5gap_A19 & GAAATCCGCGACCTGTACGTAATCTTTTTCA \\
\hline 4hb_5gap_B01 & GAACCTCCCGACTTGCGCTAACGAATGAAAA \\
\hline 4hb_5gap_B02 & TTTATTTTCATCGTAAATAATCGCAGAACGCGCCTGTT \\
\hline 4hb_5gap_B03 & CATCTTCTGACCTAAATATGCGTGCATTTTCGAGCCAG \\
\hline 4hb_5gap_B04 & AGGTTGGGTTATATATAGATTAACAATATATGTGAGTG \\
\hline 4hb_5gap_B05 & TGAATAATGGAAGGGGTACCTTTAAAAGAAGATGATGA \\
\hline 4hb_5gap_B06 & AAGAAACCACCAGAAAATTCGACAATATCTTTAGGAGC \\
\hline 4hb_5gap_B07 & GAACCCTTCTGACCTCCGAACGAAGCATCACCTTGCTG \\
\hline 4hb_5gap_B08 & TTTTGACGCTCAATCCTATCGGCAAGAGTCTGTCCATC \\
\hline 4hb_5gap_B09 & GCCCCCGATTTAGAGGCGTAACCGGAGCTAAACAGGAG \\
\hline 4hb_5gap_B10 & TCTATCAGGGCGATGTAGGGTTGGCTGGTTTGCCCCAG \\
\hline 4hb_5gap_B11 & GGTCATAGCTGTTTCCATTAATTGGGCGCCAGGGTGGT \\
\hline 4hb_5gap_B12 & GTCACGACGTTGTAAGGCCTCTTTTTCCGGCACCGCTT \\
\hline 4hb_5gap_B13 & AAGCAAATATTTAAACTGGCCTTTCACGTTGGTGTAGA \\
\hline 4hb_5gap_B14 & CAAGAGAATCGATGAATAAATTAGAGTAATGTGTAGGT \\
\hline 4hb_5gap_B15 & AACGAGTAGATTTAGTAGTAGTAACATTATGACCCTGT \\
\hline 4hb_5gap_B16 & GAATATAATGCTGTAACAGGTCATCAAAAAGATTAAGA \\
\hline 4hb_5gap_B17 & CACATTCAACTAATGAGGCTTTTTCAAATGCTTTAAAC \\
\hline 4hb_5gap_B18 & CAGGACGTTGGGAAGTGGGCTTGATATTCATTACCCAA \\
\hline 4hb_5gap_B19 & CAACCTAAGCCTGATAGAACTGACCAACTTT \\
\hline 4hb_5gap_B20 & CAGACGGTCAATCATAACTAAAGAGCCACTACGAA \\
\hline 4hb_5gap_C01 & GCCTTTACAGAACTGAACATTTGAAGCCTTAAATC \\
\hline 4hb_5gap_C02 & $\begin{array}{l}\text { AGAAAAATTATTTATAATTGAGTGAAGGCTTATCCGGT } \\
\end{array}$ \\
\hline 4hb_5gap_C03 & AAAGGTAAAGTAATTCAAAGTTAGTTAAATAAGTACCG \\
\hline 4hb_5gap_C04 & CTATTAAATTGAGAAAACGTAGAAGAACGCGAGAAAAC \\
\hline 4hb_5gap_C05 & TTACATTTAACAATTTATGGTTTAAATAAAGGGTCTGA \\
\hline 4hb_5gap_C06 & CAATAGAAGTTACAATCACCGTCGCAATTCATCAATAT \\
\hline 4hb_5gap_C07 & ATATCTGGTCAGTTGCCATCGATGGCTATTAAAAGTTT \\
\hline
\end{tabular}




\begin{tabular}{|c|c|}
\hline 4hb_5gap_C08 & TTCTTTGGCCTGCAACCCTTATTCACGACCAGTAATAA \\
\hline 4hb_5gap_C09 & ACGGTACGCCAGAATCCACCCTCGAAGAAAGGCAACAG \\
\hline 4hb_5gap_C10 & TTCCGAATGCTTTGACCTTGATAGTCGAGGTGCCGTAA \\
\hline 4hb_5gap_C11 & ACAGCTGATTGCCCTGATGATACACGAGCCGACGTGGA \\
\hline 4hb_5gap_C12 & CATTCGCCTGCATTAAACCTATTTCTAGAGGATCCCCG \\
\hline 4hb_5gap_C13 & CAGTTTGAGGGGACGGTGCCGTCTTGTTAAAGCGATTA \\
\hline 4hb_5gap_C14 & GGAGACATCGGATTCGAACCGCCGGTTGATAATCAGAA \\
\hline 4hb_5gap_C15 & TTCAACGCAAGGATAACTACAACTTTAGCTACTATCAG \\
\hline 4hb_5gap_C16 & CGTTTTATTAAGCAAGTATGGGAGGAAGTTTCATTCCA \\
\hline 4hb_5gap_C17 & ATCAAAAATCAGGTCAAAATCTCAGCAACACTCATTTT \\
\hline 4hb_5gap_C18 & GTGAATAATAGCGTCAGTTGCGCATTACAGGTAGAAAG \\
\hline 4hb_5gap_C19 & GACCAGGCGCATAGGGTCACCCTGACCCCCAATGCGAT \\
\hline 4hb_5gap_D01 & GAGAATTAGAGAATAACAATTTTATCCTGAA \\
\hline 4hb_5gap_D02 & TAATAAGAGCAAGAATAGATAAGTTTACGAGCATGTAG \\
\hline 4hb_5gap_D03 & AACCGAGGAAACGCAAATATAAAACTAGAAAAAGCCTG \\
\hline 4hb_5gap_D04 & CATAAAGGTGGCAACGCTTCTGTATCCTTGAAAACATA \\
\hline 4hb_5gap_D05 & AAAGACAAAAGGGCGCAAGAAAAACGTCAGATGAATAT \\
\hline 4hb_5gap_D06 & AGCCATTTGGGAATTTAATAGATTTAGAAGTATTAGAC \\
\hline 4hb_5gap_D07 & GTAATCAGTAGCGACTATCAAACCCTAAAACATCGCCA \\
\hline 4hb_5gap_D08 & CATCTTTTCATAATCAACCGTTGCTTGCCTGAGTAGAA \\
\hline 4hb_5gap_D09 & ACCCTCAGAGCCGCCAGGGATTTTGGCAAGTGTAGCGG \\
\hline 4hb_5gap_D10 & AAATAAATCCTCATTATCCTGTTATAAATCAAAAGAAT \\
\hline 4hb_5gap_D11 & CTGGTAATAAGTTTTCACCAGTGGTGCCTAATGAGTGA \\
\hline 4hb_5gap_D12 & CATGAAAGTATTAAGGAAACCAGCTGTTGGGAAGGGCG \\
\hline 4hb_5gap_D13 & GATATAAGTATAGCCCGTAACCGAACGCCATCAAAAAT \\
\hline 4hb_5gap_D14 & GCCACCACCCTCATTAAAGGGTGTATGATATTCAACCG \\
\hline 4hb_5gap_D15 & ATTCCACAGACAGCCGCGGGAGAAAAAGGTGGCATCAA \\
\hline 4hb_5gap_D16 & ACAACTTTCAACAGTAAAGACTTGAACCAGACCGGAAG \\
\hline 4hb_5gap_D17 & GGCTCCAAAAGGAGCAACGAGAAGACGATAAAAACCAA \\
\hline 4hb_5gap_D18 & CAACAACCATCGCCCACAAAGCTGAAACACCAGAACGA \\
\hline 4hb_5gap_D19 & AAGACAGCATCGGAACAGATGAAAGTACAACGGAGATT \\
\hline
\end{tabular}

\section{Supplementary Table 5. Staple list of 4HB designs.}




\begin{tabular}{|c|c|c|}
\hline \multicolumn{3}{|c|}{ 6HB design } \\
\hline Name & Sequence $\left(5^{\prime} \rightarrow 3^{\prime}\right)$ & Color \\
\hline 6hb_001 & GCTTGACTCACCGCCGAAAATCCTGTTTAGTTTGGCCGTCTA & \\
\hline 6hb_002 & AACGAGTATATATTCAGAAGCAAAAAAACATTATGTTTTTAG & \\
\hline 6hb_003 & GTGAAGTTTCAAAAACCATAAATCAAAAAGACTTCCCAACAG & \\
\hline 6hb_004 & GCTGAACTCACCACCAGCAGAAGATAAATAAAGCAGCAAATC & \\
\hline 6hb_005 & GAGTAGACGAGAAGTGTTTTTATAATCAAACATCACAATATT & \\
\hline 6hb_006 & CAACACTATAACAACATTATTACAGGTAAGGCATAAATAGCG & \\
\hline 6hb_007 & GGGGCGCTAAATCAAAGCTAAATCGGTTGTACCAGGCATTAA & \\
\hline 6hb_008 & CCGAACTCAAAGTACAACGGAGATTTGTCAATCATCCAGGCG & \\
\hline 6hb_009 & ACCGATAAAATAATTTTTTCACGTTGAATTAAACAACCGATA & \\
\hline 6hb_010 & TCAGGAGACCCTCAAGAGAAGGATTAGGGTGTATCCCGCCAC & \\
\hline 6hb_011 & CCCTCAGATTAGCGTTTGCCATCTTTTCCCTCAGATTGACAG & \\
\hline 6hb_012 & CAATCAACCAAGACTCCTTATTACGCAGAGTTTATGGGCGAC & \\
\hline 6hb_013 & TTTGATATTAGAGAAGAGGAAGCCCGAAATCAGGTTGTGTAG & \\
\hline 6hb_014 & TTTTTGTGTTTTTATCCTGAATCTTACCAAATAAGAATAACA & \\
\hline 6hb_015 & ATAGATATACAGTAATAAGAGAATATAACCTGTTTCGAGCAT & \\
\hline 6hb_016 & ATATAACGCAAACATAGCGATAGCTTAGGCTTAGGAGAACGC & \\
\hline 6hb_017 & GTAGATTGATTCATCAATATAATCCTGAAATAAAGCTTTTAC & \\
\hline 6hb_018 & GTCAGTAGCCAGCAATTGAGGAAGGTTACTGGGGTGCGTAAG & \\
\hline 6hb_019 & GGCAGATCTCAAATATCAAACGCTCAATCGTCTAAAAATACC & \\
\hline 6hb_020 & TAAAAGAAATACTTCAACAGGAAAAACGGCTGCATCGAGCAC & \\
\hline 6hb_021 & GCGGTCAAGAACTCAAACTAAAGGAGCGGGCGCAAGGAAGGG & \\
\hline 6hb_022 & TCAGGGCAAGTTTTGCCGGCGAACGTGGCGGGCAAGTTCCGA & \\
\hline 6hb_023 & ATAAATAATAATGCTGTAGAGACTGGATAGCGTCATAATAGT & \\
\hline 6hb_024 & AGAATTAGCAATAAAGCCTCATTGCGGGATTTCAA & \\
\hline 6hb_025 & TTGAGATATAACGCAAGAAGTTTTGCCAAGCTGATTTAATCA & \\
\hline 6hb_026 & TCAGTGAATCATAACCCTCCATTACCCAAATCAGCACAAGAA & \\
\hline 6hb_027 & AATTGTGCCGGAACCCTTCATCAAGAGTAACAAGAGTAATGC & \\
\hline 6hb_028 & AGGGTAGGACCAACTTTGATCACCCTCAGCAGCTGCGCTTTT & \\
\hline 6hb_029 & AGGCTCCCTTGCTTGGCTTGCAGGGAGTCAGGAAGACGTTAG & \\
\hline 6hb_030 & AAACTACGTTGCGCCGACAATGTACCGTAACACCCAGCCCAA & \\
\hline 6hb_031 & CATCCAAGAGCTGAGTTTGACCATTAGAATAAAAAACCCTGT & \\
\hline 6hb_032 & GCTCAGTTATAAGTCCCTCATTTTCAGGATTTTTTTCAGTGC & \\
\hline 6hb_033 & TGAATTTGTTTAGTACCGCCCTCATTAAAGCCACCTCACAAA & \\
\hline 6hb_034 & CGGAACCCGCCACCTCAGACGATTGGCCGAGTAACTCGATAG & \\
\hline 6hb_035 & TTTGGGAAGCCGCCACCAGAAGGTGAATTATCAGCCGGAAAT & \\
\hline 6hb_036 & TAGAAAAACGCAAAGGGAGGGAAGGTAATCGTAACGCCCTTT & \\
\hline 6hb_037 & AGATAACTAGAAAATTCATAAGTCAGAGGGTAAGTCTGAACA & \\
\hline 6hb_038 & TCTTTCCCCATATTCGCATTAGACGGGAGCTTCTGGGTATTC & \\
\hline 6hb_039 & GCAAGCCTTAACGTCAAAATATTAAACCAAGTACGTCATTCC & \\
\hline 6hb_040 & GTAAAGTGTTCAGCATAATCGGCTGTCTTCGCTATTTCTTAC & \\
\hline 6hb_041 & AAGGCGTAGTCCTGAACAATTAATGGTTTGAAAAAATCTTCT & \\
\hline 6hb_042 & AAGAGTCAGACTACAAATATATTTTAGTTGTAAAACCTTTTT & \\
\hline 6hb_043 & TACCTGATATATGTAAATGAAAATCGCGCAGAGAATTGAATA & \\
\hline 6hb_044 & CTTCTGAATTATTTTAACGGATTCGCCTATGGTCATAATTTT & \\
\hline 6hb_045 & TACATTTTTCAGGTTTAACAACAACTAATAGATCATATCTTT & \\
\hline 6hb_046 & GGGAGCTGTGCTTTAACCTGTCGTGCCACTCATGGTTAACCG & \\
\hline 6hb_047 & AGCCCGAAAAATCCTTTCACCAGTGAGACGAGAAACCATCAC & \\
\hline 6hb_048 & GTCCACTCAGCAGGCTGGCCCTGAGAGAGCCCCCGGCACTAA & \\
\hline 6hb_049 & GCTCATTTTACCTTTATTCAACCGTTCTGAGGGGGACTAATG & \\
\hline 6hb_050 & GCAAAAGAAGGCACGAGAGTCTGGAGCAAATCTTGTCCATGT & \\
\hline 6hb_051 & CAACTTTATTTTCTAAAAGCCCCAAAAATAAAGGCTATCGGT & \\
\hline 6hb_052 & GCCCCCTAACAGTGTGTTAAATCAGCTCGATAGCAGTCGAGA & \\
\hline 6hb_053 & TTGCCTTGTAATCACATTAAATGTGAGCTTGATATGCCTCCC & \\
\hline 6hb_054 & TACCAGAAAGTAAGTGTAGATGGGCGCAATATTGAAACATAT & \\
\hline 6hb_055 & ACTTGCGGCGAGGCAGCTTTCCGGCACCGAATTAATACAAAA & \\
\hline 6hb_056 & AATTGAGAAGCCAACGGTGCGGGCCTCTTTCCTTAACAATAA & \\
\hline 6hb_057 & AGTGAATAACAGTACCCAGTCACGACGTTAATTTCATCATAG & \\
\hline
\end{tabular}




\begin{tabular}{|c|c|}
\hline 6hb_058 & CAAAGAATGAGTAATCGAATTCGTAATCGATTGCTCCTACCA \\
\hline 6hb_059 & GCGTTTTGAGAATGGGGTGAGAAAGGCCGCAACTACTTAATT \\
\hline 6hb_060 & TATTAGTGGCACAGATAAAGTGTAAAGCTCTAAAAGTGCCAC \\
\hline 6hb_061 & TTTGCCCATTAAAGCCAACGTCAAAGGGCCGTAAAATTTAGA \\
\hline 6hb_062 & AATACTTGAGCATATACAGGCAAGGCAACTATATTCGCAAAT \\
\hline 6hb_063 & CTGACTAAAGATTAGTACCTTTACTAATAGTAGTACGGATTG \\
\hline 6hb_064 & GAGTGAGGAAAGGAGCAAATGAAAAATCACAGAGGACATCGC \\
\hline 6hb_065 & CTTTCCATGACCCTCAATCAATATCTGGGCGCTCAGGACATT \\
\hline 6hb_066 & TCGGCCAGCCATTGCTTTGATTAGTAATGTGAGGCGACAGGA \\
\hline 6hb_067 & AGGGTGGCGATCGGCCTTGCTGGTAATAGCGTATTGCTTAAT \\
\hline 6hb_068 & TTGCCCTGGGGAAATTGGGGTCGAGGTGCGAAAAAAACAAGA \\
\hline $6 \mathrm{hb} \_069$ & CACCATCTTCTCAACATGTTTTAAATATGGAGACAACAGTTC \\
\hline 6hb_070 & TGCCGGATTTGCAACAAAAGGAATTACGGAAAGATTCTACGT \\
\hline 6hb_071 & CGCAAGGTACATTTTTCATTT \\
\hline 6hb_072 & TCAGGTCTTGTTTACCAGACGACGATAAATCTACAGAACGAG \\
\hline 6hb_073 & TGAACGGTGGCTGAGAGGCGCAGACGGTATCATCGCCAGCGA \\
\hline 6hb_074 & CGGTTGACGAAGAGGACAGATGAACGGTAATCATAAGACTTT \\
\hline 6hb_075 & AAGCAAATCGCTGATCGAGGTGAATTTCAATCTCCAGGAACA \\
\hline 6hb_076 & TCGCATTCCATGACAACAACCATCGCCCTATTTTGTCATAGT \\
\hline 6hb_077 & AGGAACGGCCACCAATAGCCCGGAATAGATTAGCGCATGAAA \\
\hline 6hb_078 & CCAGCTTATCACCCTCAGAACCGCCACCTGGCCTTTTTGATG \\
\hline 6hb_079 & CGGATTCAGGCAGGCTCAGAACCGCCACATAATCAGGCATTT \\
\hline 6hb_080 & ATAGGTCTAAACCACCACCAGAGCCGCCTTGACCGCCATTAC \\
\hline 6hb_ 081 & CTGCCAGCGATTGAGACACCACGGAATATATGTTAGAATACC \\
\hline 6hb_082 & ATCGCACCAATGGTTTACCAGCGCCAAATCGGCCTAAGAGCA \\
\hline 6hb_083 & AAACCAGAGGGAAGATTTATCCCAATCCAACGCTAAGTTGCT \\
\hline 6hb_ 084 & TTGGGAAGGATGAAAATAGCAGCCTTTAAGGCTGCCCGCGCC \\
\hline 6hb_ 085 & GCTGGCGCCAATCATAATGCAGAACGCGAGTACCGTAATTTA \\
\hline 6hb_086 & AACGCCAATGAAAAATAATATCCCATCCCGATTAATTACTAG \\
\hline 6hb_087 & CAGTGCCCTTTTTCCTTTTTAACCTCCGATTAAGAAATTAAT \\
\hline 6hb_088 & CCCGGGTACCTGATGCAAATCCAATCGCGACTCTAAGAAAAC \\
\hline 6hb_ 089 & TTCCTGTGAAACAAGCACGTAAAACAGATTGTTTGTATTCCT \\
\hline 6hb_090 & TACGAGCCTGTCAGATGAATATACAGTAAATTCCAACAATTC \\
\hline 6hb_nogap_A01 & CCGGATAATTGCCTCAACCTAAACGAGAAACACCAAAGGCTA \\
\hline 6hb_nogap_A02 & CAAATAATCATCAAGTAGCGACATCATACATGGCTCCTGTAG \\
\hline 6hb_nogap_A03 & AAGAACGGGGCGATCGCTCAACATAGGAATCATTAGCAACTG \\
\hline 6hb_nogap_A04 & AGGAGCACGGAAGCACAATATTTTAGACTTTACAACACAACA \\
\hline 6hb_nogap_A05 & CATTAAAACAGAGAACATTAATTGCGTTTCAGTTGTCACCTT \\
\hline 6hb_nogap_A06 & TTATACCGGAAGTTAAAACTAGCATGTCGTACAGAAAGGGAA \\
\hline 6hb_nogap_A07 & TCGGTCAAAGGCCGGAACAAACGGCGGAGCCAGCAGCCACCA \\
\hline 6hb_nogap_A08 & GGCAGAGCTGTTTAGATGTGCTGCAAGGTAATTTAATCAACA \\
\hline 6hb_nogap_A09 & AGAAAACAATTCGAGCTTCCATTGAATCCCCCTTAAATCGTC \\
\hline 6hb_nogap_A10 & GCGCCGCGCCAGAATCCTGCTGCGCGTAACCACCAAAGTGTA \\
\hline 6hb_nogap_A11 & TAGCGTAGAATTGCGAATACGCCTGTAGCATTCACCCAGTAC \\
\hline 6hb_nogap_A12 & AGAAACAACTGGCATGATTACAAGAATTGAGTTAAATCAGAG \\
\hline 6hb_nogap_A13 & AAAATTATAGAATCCTTGAAAAAGAAGATGATGTTTTTCAAT \\
\hline 6hb_nogap_B01 & AAGAAAGTTTTTCTCTTATAAATCACACCCGCCGCGGGCGCC \\
\hline 6hb_nogap_B02 & TATTCGGTATTTAAAAAGTTTTGTCGTCAATAGAAAAAAAAA \\
\hline 6hb_nogap_B03 & CCCTGAATCCAGCCGTTTTAGCGAAGCCCAATAATCAGGAAG \\
\hline 6hb_nogap_B04 & GAGAAAAAAGCTTGTTAACAATTTCATTCGCTATTCGCTGAG \\
\hline 6hb_nogap_B05 & CCAAATCGATGGCCTTGAGTGTTGTTCCGATGGTGCAGCTGA \\
\hline 6hb_nogap_B06 & TATCAAAATAATGGGAAGGAGCGGAATTACGTTATTAGCTGT \\
\hline 6hb_nogap_B07 & GTATAACAAACAGGCATCACGCAGAAATGGATTATTCAGAGC \\
\hline 6hb_nogap_B08 & TAGTAAAACGAACTAACGGAAGGCTTGCCCTGAACTGCTCAT \\
\hline 6hb_nogap_B09 & TAAATGACAACAGTGCCTTTAATGAAAGACAGCATTGCTAAA \\
\hline 6hb_nogap_B10 & ATACAGGGAGGCTGAGACTCGTTCCAGTAAGCGGACAGTCTC \\
\hline 6hb_nogap_B11 & TTAAGAAAGGAAACATAAAGGTGCCGTCACCGACTACAAAGT \\
\hline 6hb_nogap_B12 & CAATAGCACCCAGCTACAATTTTATTTTCATCGGTAGAACAA \\
\hline
\end{tabular}




\begin{tabular}{|c|c|}
\hline 6hb_nogap_B13 & TAATGGAAACCTTGAATTTATCATACCGACCGTGTATATGTG \\
\hline 6hb_nogap_B14 & GTAAAGATTCATTCATTTTTGCGGATGGGCAAACTAAATATC \\
\hline 6hb_nogap_C01 & CCTCAGACCATCAATGGTAATAAGTTTTTCTGAAAGGGTTTT \\
\hline 6hb_nogap_C02 & ATTCAACTTTGAGGAGCAATAGCTATCTAATAACGGCAAACG \\
\hline 6hb_nogap_C03 & TAAAAACGCAAAGCTCAGATATAGAAGGCAAGATTACGAGCG \\
\hline 6hb_nogap_C04 & GTAGAAAAAAGGGGGTATCATATGCGTTCAACATGACAAAAG \\
\hline 6hb_nogap_C05 & GCTGAATTTAAAGCGAACCAGACCGGAACTTAGAGAAGTACG \\
\hline 6hb_nogap_C06 & CAGATACTTAGGAATCAGGACGTTGGGATTCAACTAAATTAA \\
\hline 6hb_nogap_C07 & TACTTAGTCGAAATTAAAACACTCATCTAAAATACGAATCGA \\
\hline 6hb_nogap_C08 & TCAGAGCAGAGCCAAGACTGTAGCGCGTGAAACCAAACCCGT \\
\hline 6hb_nogap_C09 & GTCTGAGAATAGTGCTTCTGTAAATCGTTGAATTACGACGGC \\
\hline 6hb_nogap_C10 & GCTGAGATTAACACGCGCGAACTGATAGCCTGAAAGCCTAAT \\
\hline 6hb_nogap_C11 & CATCAAATTATAGTTTAAATGCAATGCCTTCCCAATGCTCCT \\
\hline 6hb_nogap_C12 & AATCGGCGATAGGGCACTACGTGTAGGGCGCTGGCAAAGAAT \\
\hline 6hb_nogap_C13 & CTTGAGTGCCTATTGGATAAGTGTGAGTTTCGTCAAGTTAAT \\
\hline 6hb_nogap_C14 & AATACGTCTTTAATCGCCTGCAATAGAGCCGTCAAGAATGGC \\
\hline 6hb_nogap_C15 & AACCCTCAGATTTAAAAGGTGGCATCAATTCTAATTTCTGCG \\
\hline 6hb_nogap_D01 & GAGGTTGTCCGTGGGAAACGTCACCAATTTTCATCAAATCAC \\
\hline 6hb_nogap_D02 & ATCGGGAGTGAAATAATCCTTTGCCCGAATCATCAGATTATA \\
\hline 6hb_nogap_D03 & TTGTAGCGTCTGTCAGGCCGATTAAAGGGCTTTGATAATGAA \\
\hline 6hb_nogap_D04 & TTATCAGAAAAGGATTCAGCGGAGTGAGTTTCCAGATTGTAT \\
\hline 6hb_nogap_D05 & CTGGCCAAATACCGAACGAACCAGTCACACGACAATTACATT \\
\hline 6hb_nogap_D06 & TTGTGAAATACCAGTACCACATTCCAATACTGCGGAGAACTG \\
\hline 6hb_nogap_D07 & CACTACGAATACACCCGCGACCTACGTAACAAAGCGAAAGAG \\
\hline 6hb_nogap_D08 & TTCATGAAAGCGCGAAACAACGGCTACAGAGGCTTCGGAACG \\
\hline 6hb_nogap_D09 & CAGCACCTAGCGTCCCACCGGAAGAATGGAAAGCGATCAAGT \\
\hline 6hb_nogap_D10 & TAAGAACGGAGGTTAATTTGCCATTGAGCGCTAATCCTCCCG \\
\hline 6hb_nogap_D11 & CAGTATAAATCGCCTCCAGACGACCGCACTCATCGAGGGCTT \\
\hline 6hb_nogap_D12 & AAAAAGCGCATTTTCGAGCAATAAGAATAAACAATGATAAAT \\
\hline 6hb_nogap_D13 & AAAAGTTACCACCAAAGGGTTAGGCGAATTATTCATGCGGAA \\
\hline 6hb_nogap_E01 & AACAGTTCTAACTCTAGAACCCTTCTGACCCTAAATGAGGCG \\
\hline 6hb_nogap_E02 & ACCGCCAACGCGCGCGCGTACTATGGTTGATTTTACACCGAG \\
\hline 6hb_nogap_E03 & AGAGGCTGAGGGTATGAGATGGTTTAATAGAAAAATCATCAG \\
\hline 6hb_nogap_E04 & CATAGGCTAATCGTTCCATTAAACGGGTTTGACCCCCTGATA \\
\hline 6hb_nogap_E05 & TAGGAACAAATTTTCCCGTATAACACAGACAGCCCTTAAAAT \\
\hline 6hb_nogap_E06 & CCAAGTTACCGAGCCATTATCATAAACAAACATCAGAGGATC \\
\hline 6hb_nogap_E07 & TAAACAGAGAGCCTTTGAAGCCTTAAATCTTATCCGTGCCGG \\
\hline 6hb_nogap_E08 & ACAACATAATTCTGATATTTAACAACGCATACAAATACGCCA \\
\hline 6hb_nogap_E09 & GTCAGGAAGAGGTCCATATAACAGTTGATGAGTAACTTTACC \\
\hline 6hb_nogap_E10 & GTATTAAAGTGTACAAATAATTCGCGTCCTCAGAAACCGTAC \\
\hline 6hb_nogap_E11 & CAAAAGAATGAAATGGACGACGACAGTAGACAAAATTTGTCA \\
\hline 6hb_nogap_E12 & CATTAGCTAGCCCCCTTATTAGAGCCAGCAAAAGATGAGCCA \\
\hline 6hb_nogap_E13 & GACAACTAGATGATGGCAAGGATTTAGAAGTATTTTAGATAA \\
\hline 6hb_nogap_F01 & TACATTTGTCGGGACCTCGTTAGCAGTAATAAAAGCTGCCCG \\
\hline 6hb_nogap_F02 & AAAATGTAATATGAATGCGATTTCAAATGCTTTAAGTCAAAT \\
\hline 6hb_nogap_F03 & GCGGGATTAATCAGGTATGGGATTTTGAGGACTAATGTACCC \\
\hline 6hb_nogap_F04 & TATTCATACGTTGGCAGATAGCCTCACCAGTAGCATAATGGG \\
\hline 6hb_nogap_F05 & GACCTAAGGGTTTTCATAAATCACCGGAATCATAAGTTGGGT \\
\hline 6hb_nogap_F06 & GGGTTGAACCAGGCTCGGAACCTATTATAACGGGGAACCAAT \\
\hline 6hb_nogap_F07 & AAAAGAATACATACCGAGGAAACGCAATTACCGAACGTGCAT \\
\hline 6hb_nogap_F08 & ACGGTACTACAGGGGGGAGAGGCGGTTTTCCAGAACTTGCCT \\
\hline 6hb_nogap_F09 & TAATAAATTGGGCTGCTATTTTTGAGAGAAACCAAGTAAGAG \\
\hline 6hb_nogap_F10 & ACTAAAGACGATCTATTGTAAACGTTAAACGCATAGCTTGAT \\
\hline 6hb_nogap_F11 & ATTTTGCAAGCAAAGCCATTCGCCATTCCAGAGAGAAACGAT \\
\hline 6hb_nogap_F12 & TTTCCCTATTACATCATGCCTGCAGGTCAAGACAATTGGGTT \\
\hline 6hb_nogap_F13 & GATTATCCGTATTATGTTATCCGCTCACACAGTACAAATTGC \\
\hline 6hb_1gap_A01 & CGGATAATTGCCTCAACCTAAACGAGAAACACCAAAGGCT \\
\hline
\end{tabular}




\begin{tabular}{|c|c|}
\hline 6hb_1gap_A02 & AAATAATCATCAAGTAGCGACATCATACATGGCTCCTGTA \\
\hline 6hb_1gap_A03 & AGAACGGGGCGATCGCTCAACATAGGAATCATTAGCAACT \\
\hline 6hb_1gap_A04 & GGAGCACGGAAGCACAATATTTTAGACTTTACAACACAAC \\
\hline 6hb_1gap_A05 & ATTAAAACAGAGAACATTAATTGCGTTTCAGTTGTCACCT \\
\hline 6hb_1gap_A06 & $\begin{array}{l}\text { TATACCGGAAGTTAAAACTAGCATGTCGTACAGAAAGGGA } \\
\end{array}$ \\
\hline 6hb_1gap_A07 & CGGTCAAAGGCCGGAACAAACGGCGGAGCCAGCAGCCACC \\
\hline 6hb_1gap_A08 & GCAGAGCTGTTTAGATGTGCTGCAAGGTAATTTAATCAAC \\
\hline 6hb_1gap_A09 & GAAAACAATTCGAGCTTCCATTGAATCCCCCTTAAATCGT \\
\hline 6hb_1gap_A10 & CGCCGCGCCAGAATCCTGCTGCGCGTAACCACCAAAGTGT \\
\hline 6hb_1gap_A11 & AGCGTAGAATTGCGAATACGCCTGTAGCATTCACCCAGTA \\
\hline 6hb_1gap_A12 & GAAACAACTGGCATGATTACAAGAATTGAGTTAAATCAGA \\
\hline 6hb_1gap_A13 & AAATTATAGAATCCTTGAAAAAGAAGATGATGTTTTTCAA \\
\hline 6hb_1gap2_A01 & TTGATATTAGAGAAGAGGAAGCCCGAAATCAGGTTGTGTA \\
\hline 6hb_1gap2_A02 & AGTAGACGAGAAGTGTTTTTATAATCAAACATCACAATAT \\
\hline 6hb_1gap2_A03 & $\begin{array}{l}\text { CGAACTCAAAGTACAACGGAGATTTGTCAATCATCCAGGC } \\
\end{array}$ \\
\hline 6hb_1gap2_A04 & TAGATATACAGTAATAAGAGAATATAACCTGTTTCGAGCA \\
\hline 6hb_1gap2_A05 & TAGATTGATTCATCAATATAATCCTGAAATAAAGCTTTTA \\
\hline 6hb_1gap2_A06 & GCAGATCTCAAATATCAAACGCTCAATCGTCTAAAAATAC \\
\hline 6hb_1gap2_A07 & AACTACGTTGCGCCGACAATGTACCGTAACACCCAGCCCA \\
\hline 6hb_1gap2_A08 & TTGGGAAGCCGCCACCAGAAGGTGAATTATCAGCCGGAAA \\
\hline 6hb_1gap2_A09 & СТTTCCCCATATTCGCATTAGACGGGAGCTTCTGGGTATT \\
\hline 6hb_1gap2_A10 & CAAAAGAAGGCACGAGAGTCTGGAGCAAATCTTGTCCATG \\
\hline 6hb_1gap2_A11 & GGAACGGCCACCAATAGCCCGGAATAGATTAGCGCATGAA \\
\hline 6hb_1gap2_A12 & TCGCACCAATGGTTTACCAGCGCCAAATCGGCCTAAGAGC \\
\hline 6hb_1gap2_A13 & AGTGCCCTTTTTCCTTTTTAACCTCCGATTAAGAAATTAA \\
\hline 6hb_1gap_B01 & AGAAAGTTTTTCTCTTATAAATCACACCCGCCGCGGGCGC \\
\hline 6hb_1gap_B02 & ATTCGGTATTTAAAAAGTTTTGTCGTCAATAGAAAAAAAA \\
\hline 6hb_1gap_B03 & CCTGAATCCAGCCGTTTTAGCGAAGCCCAATAATCAGGAA \\
\hline 6hb_1gap_B04 & AGAAAAAAGCTTGTTAACAATTTCATTCGCTATTCGCTGA \\
\hline 6hb_1gap_B05 & CAAATCGATGGCCTTGAGTGTTGTTCCGATGGTGCAGCTG \\
\hline 6hb_1gap_B06 & ATCAAAATAATGGGAAGGAGCGGAATTACGTTATTAGCTG \\
\hline 6hb_1gap_B07 & TATAACAAACAGGCATCACGCAGAAATGGATTATTCAGAG \\
\hline 6hb_1gap_B08 & AGTAAAACGAACTAACGGAAGGCTTGCCCTGAACTGCTCA \\
\hline 6hb_1gap_B09 & AAATGACAACAGTGCCTTTAATGAAAGACAGCATTGCTAA \\
\hline 6hb_1gap_B10 & TACAGGGAGGCTGAGACTCGTTCCAGTAAGCGGACAGTCT \\
\hline 6hb_1gap_B11 & TAAGAAAGGAAACATAAAGGTGCCGTCACCGACTACAAAG \\
\hline 6hb_1gap_B12 & AATAGCACCCAGCTACAATTTTATTTTCATCGGTAGAACA \\
\hline 6hb_1gap_B13 & AATGGAAACCTTGAATTTATCATACCGACCGTGTATATGT \\
\hline 6hb_1gap_B14 & TAAAGATTCATTCATTTTTGCGGATGGGCAAACTAAATAT \\
\hline 6hb_1gap2_B01 & AACACTATAACAACATTATTACAGGTAAGGCATAAATAGC \\
\hline 6hb_1gap2_B02 & TCAGTAGCCAGCAATTGAGGAAGGTTACTGGGGTGCGTAA \\
\hline 6hb_1gap2_B03 & GGCTCCCTTGCTTGGCTTGCAGGGAGTCAGGAAGACGTTA \\
\hline 6hb_1gap2_B04 & AGAAAAACGCAAAGGGAGGGAAGGTAATCGTAACGCCCTT \\
\hline 6hb_1gap2_B05 & CAAGCCTTAACGTCAAAATATTAAACCAAGTACGTCATTC \\
\hline 6hb_1gap2_B06 & AGGCGTAGTCCTGAACAATTAATGGTTTGAAAAAATCTTC \\
\hline 6hb_1gap2_B07 & GCCCGAAAAATCCTTTCACCAGTGAGACGAGAAACCATCA \\
\hline 6hb_1gap2_B08 & TGCCTTGTAATCACATTAAATGTGAGCTTGATATGCCTCC \\
\hline 6hb_1gap2_B09 & CGTTTTGAGAATGGGGTGAGAAAGGCCGCAACTACTTAAT \\
\hline 6hb_1gap2_B10 & TGCCCTGGGGAAATTGGGGTCGAGGTGCGAAAAAAACAAGA \\
\hline 6hb_1gap2_B11 & GGTTGACGAAGAGGACAGATGAACGGTAATCATAAGACTT \\
\hline 6hb_1gap2_B12 & TAGGTCTAAACCACCACCAGAGCCGCCTTGACCGCCATTA \\
\hline 6hb_1gap2_B13 & CCGGGTACCTGATGCAAATCCAATCGCGACTCTAAGAAAA \\
\hline 6hb_1gap2_B14 & ACGAGCCTGTCAGATGAATATACAGTAAATTCCAACAATT \\
\hline 6hb_1gap_C01 & CTCAGACCATCAATGGTAATAAGTTTTTCTGAAAGGGTTT \\
\hline 6hb_1gap_C02 & TTCAACTTTGAGGAGCAATAGCTATCTAATAACGGCAAAC \\
\hline 6hb_1gap_C03 & AAAAACGCAAAGCTCAGATATAGAAGGCAAGATTACGAGC \\
\hline
\end{tabular}




\begin{tabular}{|c|c|c|}
\hline 6hb_1gap_C04 & TAGAAAAAAGGGGGTATCATATGCGTTCAACATGACAAAA & \\
\hline 6hb_1gap_C05 & CTGAATTTAAAGCGAACCAGACCGGAACTTAGAGAAGTAC & \\
\hline 6hb_1gap_C06 & AGATACTTAGGAATCAGGACGTTGGGATTCAACTAAATTA & \\
\hline 6hb_1gap_C07 & ACTTAGTCGAAATTAAAACACTCATCTAAAATACGAATCG & \\
\hline 6hb_1gap_C08 & CAGAGCAGAGCCAAGACTGTAGCGCGTGAAACCAAACCCG & \\
\hline 6hb_1gap_C09 & TCTGAGAATAGTGCTTCTGTAAATCGTTGAATTACGACGG & \\
\hline 6hb_1gap_C10 & CTGAGATTAACACGCGCGAACTGATAGCCTGAAAGCCTAA & \\
\hline 6hb_1gap_C11 & ATCAAATTATAGTTTAAATGCAATGCCTTCCCAATGCTCC & \\
\hline 6hb_1gap_C12 & ATCGGCGATAGGGCACTACGTGTAGGGCGCTGGCAAAGAA & \\
\hline 6hb_1gap_C13 & TTGAGTGCCTATTGGATAAGTGTGAGTTTCGTCAAGTTAA & \\
\hline 6hb_1gap_C14 & ATACGTCTTTAATCGCCTGCAATAGAGCCGTCAAGAATGG & \\
\hline 6hb_1gap_C15 & ACCCTCAGATTTAAAAGGTGGCATCAATTCTAATTTCTGC & \\
\hline 6hb_1gap2_C01 & TGAAGTTTCAAAAACCATAAATCAAAAAGACTTCCCAACA & \\
\hline 6hb_1gap2_C02 & CCGATAAAATAATTTTTTCACGTTGAATTAAACAACCGAT & \\
\hline 6hb_1gap2_C03 & TTTTGTGTTTTTATCCTGAATCTTACCAAATAAGAATAAC & \\
\hline 6hb_1gap2_C04 & TATAACGCAAACATAGCGATAGCTTAGGCTTAGGAGAACG & \\
\hline 6hb_1gap2_C05 & TCAGGGCAAGTTTTGCCGGCGAACGTGGCGGGCAAGTTCCG & \\
\hline 6hb_1gap2_C06 & GAATTTGTTTAGTACCGCCCTCATTAAAGCCACCTCACAA & \\
\hline 6hb_1gap2_C07 & GATAACTAGAAAATTCATAAGTCAGAGGGTAAGTCTGAAC & \\
\hline 6hb_1gap2_C08 & GTGAATAACAGTACCCAGTCACGACGTTAATTTCATCATA & \\
\hline 6hb_1gap2_C09 & ATTAGTGGCACAGATAAAGTGTAAAGCTCTAAAAGTGCCA & \\
\hline 6hb_1gap2_C10 & TTTCCATGACCCTCAATCAATATCTGGGCGCTCAGGACAT & \\
\hline 6hb_1gap2_C11 & CGGCCAGCCATTGCTTTGATTAGTAATGTGAGGCGACAGG & \\
\hline 6hb_1gap2_C12 & CAGGTCTTGTTTACCAGACGACGATAAATCTACAGAACGA & \\
\hline 6hb_1gap2_C13 & GAACGGTGGCTGAGAGGCGCAGACGGTATCATCGCCAGCG & \\
\hline 6hb_1gap2_C14 & GGATTCAGGCAGGCTCAGAACCGCCACATAATCAGGCATT & \\
\hline 6hb_1gap_D01 & AGGTTGTCCGTGGGAAACGTCACCAATTTTCATCAAATCA & \\
\hline 6hb_1gap_D02 & TCGGGAGTGAAATAATCCTTTGCCCGAATCATCAGATTAT & \\
\hline 6hb_1gap_D03 & TGTAGCGTCTGTCAGGCCGATTAAAGGGCTTTGATAATGA & \\
\hline 6hb_1gap_D04 & TATCAGAAAAGGATTCAGCGGAGTGAGTTTCCAGATTGTA & \\
\hline 6hb_1gap_D05 & TGGCCAAATACCGAACGAACCAGTCACACGACAATTACAT & \\
\hline 6hb_1gap_D06 & TGTGAAATACCAGTACCACATTCCAATACTGCGGAGAACT & \\
\hline 6hb_1gap_D07 & ACTACGAATACACCCGCGACCTACGTAACAAAGCGAAAGA & \\
\hline 6hb_1gap_D08 & TCATGAAAGCGCGAAACAACGGCTACAGAGGCTTCGGAAC & \\
\hline 6hb_1gap_D09 & AGCACCTAGCGTCCCACCGGAAGAATGGAAAGCGATCAAG & \\
\hline 6hb_1gap_D10 & AAGAACGGAGGTTAATTTGCCATTGAGCGCTAATCCTCCC & \\
\hline 6hb_1gap_D11 & AGTATAAATCGCCTCCAGACGACCGCACTCATCGAGGGCT & \\
\hline 6hb_1gap_D12 & AAAAGCGCATTTTCGAGCAATAAGAATAAACAATGATAAA & \\
\hline 6hb_1gap_D13 & AAAGTTACCACCAAAGGGTTAGGCGAATTATTCATGCGGA & \\
\hline 6hb_1gap2_D01 & CTGAACTCACCACCAGCAGAAGATAAATAAAGCAGCAAAT & \\
\hline 6hb_1gap2_D02 & AATCAACCAAGACTCCTTATTACGCAGAGTTTATGGGCGA & \\
\hline 6hb_1gap2_D03 & CGGTCAAGAACTCAAACTAAAGGAGCGGGCGCAAGGAAGG & \\
\hline 6hb_1gap2_D04 & TGAGATATAACGCAAGAAGTTTTGCCAAGCTGATTTAATC & \\
\hline 6hb_1gap2_D05 & GGGTAGGACCAACTTTGATCACCCTCAGCAGCTGCGCTTT & \\
\hline 6hb_1gap2_D06 & GGAACCCGCCACCTCAGACGATTGGCCGAGTAACTCGATA & \\
\hline 6hb_1gap2_D07 & AGAGTCAGACTACAAATATATTTTAGTTGTAAAACCTTTT & \\
\hline 6hb_1gap2_D08 & ACATTTTTCAGGTTTAACAACAACTAATAGATCATATCTT & \\
\hline 6hb_1gap2_D09 & СТCATTTTACCTTTATTCAACCGTTCTGAGGGGGACTAAT & \\
\hline 6hb_1gap2_D10 & CCCCCTAACAGTGTGTTAAATCAGCTCGATAGCAGTCGAG & \\
\hline 6hb_1gap2_D11 & ATTGAGAAGCCAACGGTGCGGGCCTCTTTCCTTAACAATA & \\
\hline 6hb_1gap2_D12 & TGACTAAAGATTAGTACCTTTACTAATAGTAGTACGGATT & \\
\hline 6hb_1gap2_D13 & CAGCTTATCACCCTCAGAACCGCCACCTGGCCTTTTTGAT & \\
\hline 6hb_1gap2_D14 & CTGGCGCCAATCATAATGCAGAACGCGAGTACCGTAATTT & \\
\hline 6hb_1gap_E01 & ACAGTTCTAACTCTAGAACCCTTCTGACCCTAAATGAGGC & \\
\hline 6hb_1gap_E02 & CCGCCAACGCGCGCGCGTACTATGGTTGATTTTACACCGA & \\
\hline 6hb_1gap_E03 & GAGGCTGAGGGTATGAGATGGTTTAATAGAAAAATCATCA & \\
\hline 6hb_1gap_E04 & ATAGGCTAATCGTTCCATTAAACGGGTTTGACCCCCTGAT & \\
\hline 6hb_1gap_E05 & AGGAACAAATTTTCCCGTATAACACAGACAGCCCTTAAAA & \\
\hline
\end{tabular}




\begin{tabular}{|c|c|}
\hline 6hb_1gap_E06 & CAAGTTACCGAGCCATTATCATAAACAAACATCAGAGGAT \\
\hline 6hb_1gap_E07 & AAACAGAGAGCCTTTGAAGCCTTAAATCTTATCCGTGCCG \\
\hline 6hb_1gap_E08 & CAACATAATTCTGATATTTAACAACGCATACAAATACGCC \\
\hline 6hb_1gap_E09 & TCAGGAAGAGGTCCATATAACAGTTGATGAGTAACTTTAC \\
\hline 6hb_1gap_E10 & TATTAAAGTGTACAAATAATTCGCGTCCTCAGAAACCGTA \\
\hline 6hb_1gap_E11 & AAAAGAATGAAATGGACGACGACAGTAGACAAAATTTGTC \\
\hline 6hb_1gap_E12 & ATTAGCTAGCCCCCTTATTAGAGCCAGCAAAAGATGAGCC \\
\hline 6hb_1gap_E13 & ACAACTAGATGATGGCAAGGATTTAGAAGTATTTTAGATA \\
\hline 6hb_1gap2_E01 & CAGGAGACCCTCAAGAGAAGGATTAGGGTGTATCCCGCCA \\
\hline 6hb_1gap2_E02 & CAGTGAATCATAACCCTCCATTACCCAAATCAGCACAAGA \\
\hline 6hb_1gap2_E03 & ATTGTGCCGGAACCCTTCATCAAGAGTAACAAGAGTAATG \\
\hline 6hb_1gap2_E04 & ACCTGATATATGTAAATGAAAATCGCGCAGAGAATTGAAT \\
\hline 6hb_1gap2_E05 & GGAGCTGTGCTTTAACCTGTCGTGCCACTCATGGTTAACC \\
\hline 6hb_1gap2_E06 & ACCAGAAAGTAAGTGTAGATGGGCGCAATATTGAAACATA \\
\hline 6hb_1gap2_E07 & GGGTGGCGATCGGCCTTGCTGGTAATAGCGTATTGCTTAA \\
\hline 6hb_1gap2_E08 & ACCATCTTCTCAACATGTTTTAAATATGGAGACAACAGTT \\
\hline 6hb_1gap2_E09 & CGCATTCCATGACAACAACCATCGCCCTATTTTGTCATAG \\
\hline 6hb_1gap2_E10 & TGCCAGCGATTGAGACACCACGGAATATATGTTAGAATAC \\
\hline 6hb_1gap2_E11 & AACCAGAGGGAAGATTTATCCCAATCCAACGCTAAGTTGC \\
\hline 6hb_1gap2_E12 & ACGCCAATGAAAAATAATATCCCATCCCGATTAATTACTA \\
\hline 6hb_1gap2_E13 & TCCTGTGAAACAAGCACGTAAAACAGATTGTTTGTATTCC \\
\hline 6hb_1gap_F01 & ACATTTGTCGGGACCTCGTTAGCAGTAATAAAAGCTGCCC \\
\hline 6hb_1gap_F02 & AAATGTAATATGAATGCGATTTCAAATGCTTTAAGTCAAA \\
\hline 6hb_1gap_F03 & CGGGATTAATCAGGTATGGGATTTTGAGGACTAATGTACC \\
\hline 6hb_1gap_F04 & ATTCATACGTTGGCAGATAGCCTCACCAGTAGCATAATGG \\
\hline 6hb_1gap_F05 & ACCTAAGGGTTTTCATAAATCACCGGAATCATAAGTTGGG \\
\hline 6hb_1gap_F06 & GGTTGAACCAGGCTCGGAACCTATTATAACGGGGAACCAA \\
\hline 6hb_1gap_F07 & AAAGAATACATACCGAGGAAACGCAATTACCGAACGTGCA \\
\hline 6hb_1gap_F08 & CGGTACTACAGGGGGGAGAGGCGGTTTTCCAGAACTTGCC \\
\hline 6hb_1gap_F09 & AATAAATTGGGCTGCTATTTTTGAGAGAAACCAAGTAAGA \\
\hline 6hb_1gap_F10 & CTAAAGACGATCTATTGTAAACGTTAAACGCATAGCTTGA \\
\hline 6hb_1gap_F11 & TTTTGCAAGCAAAGCCATTCGCCATTCCAGAGAGAAACGA \\
\hline 6hb_1gap_F12 & TTCCCTATTACATCATGCCTGCAGGTCAAGACAATTGGGT \\
\hline 6hb_1gap_F13 & ATTATCCGTATTATGTTATCCGCTCACACAGTACAAATTG \\
\hline 6hb_1gap2_F01 & ACGAGTATATATTCAGAAGCAAAAAAACATTATGTTTTTA \\
\hline 6hb_1gap2_F02 & CCTCAGATTAGCGTTTGCCATCTTTTCCCTCAGATTGACA \\
\hline 6hb_1gap2_F03 & AAAAGAAATACTTCAACAGGAAAAACGGCTGCATCGAGCA \\
\hline 6hb_1gap2_F04 & TAAATAATAATGCTGTAGAGACTGGATAGCGTCATAATAG \\
\hline 6hb_1gap2_F05 & CTCAGTTATAAGTCCCTCATTTTCAGGATTTTTTTCAGTG \\
\hline 6hb_1gap2_F06 & TAAAGTGTTCAGCATAATCGGCTGTCTTCGCTATTTCTTA \\
\hline 6hb_1gap2_F07 & TTCTGAATTATTTTAACGGATTCGCCTATGGTCATAATTT \\
\hline 6hb_1gap2_F08 & AACTTTATTTTCTAAAAGCCCCAAAAATAAAGGCTATCGG \\
\hline 6hb_1gap2_F09 & CTTGCGGCGAGGCAGCTTTCCGGCACCGAATTAATACAAA \\
\hline 6hb_1gap2_F10 & AAAGAATGAGTAATCGAATTCGTAATCGATTGCTCCTACC \\
\hline 6hb_1gap2_F11 & AGTGAGGAAAGGAGCAAATGAAAAATCACAGAGGACATCG \\
\hline 6hb_1gap2_F12 & GCCGGATTTGCAACAAAAGGAATTACGGAAAGATTCTACG \\
\hline 6hb_1gap2_F13 & AGCAAATCGCTGATCGAGGTGAATTTCAATCTCCAGGAAC \\
\hline 6hb_1gap2_F14 & TGGGAAGGATGAAAATAGCAGCCTTTAAGGCTGCCCGCGC \\
\hline 6hb_2gap_A01 & GGATAATTGCCTCAACCTAAACGAGAAACACCAAAGGC \\
\hline 6hb_2gap_A02 & AATAATCATCAAGTAGCGACATCATACATGGCTCCTGT \\
\hline 6hb_2gap_A03 & GAACGGGGCGATCGCTCAACATAGGAATCATTAGCAAC \\
\hline 6hb_2gap_A04 & GAGCACGGAAGCACAATATTTTAGACTTTACAACACAA \\
\hline 6hb_2gap_A05 & TTAAAACAGAGAACATTAATTGCGTTTCAGTTGTCACC \\
\hline 6hb_2gap_A06 & ATACCGGAAGTTAAAACTAGCATGTCGTACAGAAAGGG \\
\hline 6hb_2gap_A07 & GGTCAAAGGCCGGAACAAACGGCGGAGCCAGCAGCCAC \\
\hline 6hb_2gap_A08 & CAGAGCTGTTTAGATGTGCTGCAAGGTAATTTAATCAA \\
\hline 6hb_2gap_A09 & AAAACAATTCGAGCTTCCATTGAATCCCCCTTAAATCG \\
\hline
\end{tabular}




\begin{tabular}{|c|c|}
\hline 6hb_2gap_A10 & GCCGCGCCAGAATCCTGCTGCGCGTAACCACCAAAGTG \\
\hline 6hb_2gap_A11 & GCGTAGAATTGCGAATACGCCTGTAGCATTCACCCAGT \\
\hline 6hb_2gap_A12 & AAACAACTGGCATGATTACAAGAATTGAGTTAAATCAG \\
\hline 6hb_2gap_A13 & AATTATAGAATCCTTGAAAAAGAAGATGATGTTTTTCA \\
\hline 6hb_2gap2_A01 & TGAACTCACCACCAGCAGAAGATAAATAAAGCAGCAAATC \\
\hline 6hb_2gap2_A02 & GAACTCAAAGTACAACGGAGATTTGTCAATCATCCAGGCG \\
\hline 6hb_2gap2_A03 & ACCGATAAAATAATTTTTTCACGTTGAATTAAACAACCGA \\
\hline 6hb_2gap2_A04 & CTCAGATTAGCGTTTGCCATCTTTTCCCTCAGATTGACAG \\
\hline 6hb_2gap2_A05 & AGATATACAGTAATAAGAGAATATAACCTGTTTCGAGCAT \\
\hline 6hb_2gap2_A06 & GGTCAAGAACTCAAACTAAAGGAGCGGGCGCAAGGAAGGG \\
\hline 6hb_2gap2_A07 & AAATAATAATGCTGTAGAGACTGGATAGCGTCATAATAGT \\
\hline 6hb_2gap2_A08 & TGCCGGATTTGCAACAAAAGGAATTACGGAAAGATTCTAC \\
\hline 6hb_2gap2_A09 & ACTACGTTGCGCCGACAATGTACCGTAACACCCAGCCCAA \\
\hline 6hb_2gap2_A10 & TGAATTTGTTTAGTACCGCCCTCATTAAAGCCACCTCACA \\
\hline 6hb_2gap2_A11 & ATAACTAGAAAATTCATAAGTCAGAGGGTAAGTCTGAACA \\
\hline 6hb_2gap2_A12 & GCAAGCCTTAACGTCAAAATATTAAACCAAGTACGTCATT \\
\hline 6hb_2gap2_A13 & CCTGATATATGTAAATGAAAATCGCGCAGAGAATTGAATA \\
\hline 6hb_2gap2_A14 & TACATTTTTCAGGTTTAACAACAACTAATAGATCATATCT \\
\hline 6hb_2gap2_A15 & GAGTGAGGAAAGGAGCAAATGAAAAATCACAGAGGACATC \\
\hline 6hb_2gap2_A16 & AGGGTGGCGATCGGCCTTGCTGGTAATAGCGTATTGCTTA \\
\hline 6hb_2gap2_A17 & CACCATCTTCTCAACATGTTTTAAATATGGAGACAACAGT \\
\hline 6hb_2gap2_A18 & AGGTCTTGTTTACCAGACGACGATAAATCTACAGAACGAG \\
\hline 6hb_2gap2_A19 & TGAACGGTGGCTGAGAGGCGCAGACGGTATCATCGCCAGC \\
\hline 6hb_2gap2_A20 & AGCTTATCACCCTCAGAACCGCCACCTGGCCTTTTTGATG \\
\hline 6hb_2gap2_A21 & CGGATTCAGGCAGGCTCAGAACCGCCACATAATCAGGCAT \\
\hline 6hb_2gap2_A22 & ATCGCACCAATGGTTTACCAGCGCCAAATCGGCCTAAGAG \\
\hline 6hb_2gap2_A23 & ACCAGAGGGAAGATTTATCCCAATCCAACGCTAAGTTGCT \\
\hline 6hb_2gap2_A24 & GCTGGCGCCAATCATAATGCAGAACGCGAGTACCGTAATT \\
\hline 6hb_2gap2_A25 & CCCGGGTACCTGATGCAAATCCAATCGCGACTCTAAGAAA \\
\hline 6hb_2gap2_A26 & CGAGCCTGTCAGATGAATATACAGTAAATTCCAACAATTC \\
\hline 6hb_2gap_B01 & GAAAGTTTTTCTCTTATAAATCACACCCGCCGCGGGCG \\
\hline 6hb_2gap_B02 & TTCGGTATTTAAAAAGTTTTGTCGTCAATAGAAAAAAA \\
\hline 6hb_2gap_B03 & CTGAATCCAGCCGTTTTAGCGAAGCCCAATAATCAGGA \\
\hline 6hb_2gap_B04 & GAAAAAAGCTTGTTAACAATTTCATTCGCTATTCGCTG \\
\hline 6hb_2gap_B05 & AAATCGATGGCCTTGAGTGTTGTTCCGATGGTGCAGCT \\
\hline 6hb_2gap_B06 & TCAAAATAATGGGAAGGAGCGGAATTACGTTATTAGCT \\
\hline 6hb_2gap_B07 & ATAACAAACAGGCATCACGCAGAAATGGATTATTCAGA \\
\hline 6hb_2gap_B08 & GTAAAACGAACTAACGGAAGGCTTGCCCTGAACTGCTC \\
\hline 6hb_2gap_B09 & AATGACAACAGTGCCTTTAATGAAAGACAGCATTGCTA \\
\hline 6hb_2gap_B10 & ACAGGGAGGCTGAGACTCGTTCCAGTAAGCGGACAGTC \\
\hline 6hb_2gap_B11 & AAGAAAGGAAACATAAAGGTGCCGTCACCGACTACAAA \\
\hline 6hb_2gap_B12 & ATAGCACCCAGCTACAATTTTATTTTCATCGGTAGAAC \\
\hline 6hb_2gap_B13 & ATGGAAACCTTGAATTTATCATACCGACCGTGTATATG \\
\hline 6hb_2gap_B14 & AAAGATTCATTCATTTTTGCGGATGGGCAAACTAAATA \\
\hline 6hb_2gap2_B01 & $\begin{array}{l}\text { GAGTAGACGAGAAGTGTTTTTATAATCAAACATCACAATA } \\
\end{array}$ \\
\hline 6hb_2gap2_B02 & CAATCAACCAAGACTCCTTATTACGCAGAGTTTATGGGCG \\
\hline 6hb_2gap2_B03 & TTTGATATTAGAGAAGAGGAAGCCCGAAATCAGGTTGTGT \\
\hline 6hb_2gap2_B04 & ATATAACGCAAACATAGCGATAGCTTAGGCTTAGGAGAAC \\
\hline 6hb_2gap2_B05 & AAAGAAATACTTCAACAGGAAAAACGGCTGCATCGAGC \\
\hline 6hb_2gap2_B06 & GCGGTCAAGAACTCAAACTAAAGGAGCGGGCGCAAGGAAG \\
\hline 6hb_2gap2_B07 & CCGGATTTGCAACAAAAGGAATTACGGAAAGATTCTACGT \\
\hline 6hb_2gap2_B08 & GCTCCCTTGCTTGGCTTGCAGGGAGTCAGGAAGACGTT \\
\hline 6hb_2gap2_B09 & AAACTACGTTGCGCCGACAATGTACCGTAACACCCAGCCC \\
\hline 6hb_2gap2_B10 & AATTTGTTTAGTACCGCCCTCATTAAAGCCACCTCACAAA \\
\hline 6hb_2gap2_B11 & GAAAAACGCAAAGGGAGGGAAGGTAATCGTAACGCCCT \\
\hline 6hb_2gap2_B12 & AGATAACTAGAAAATTCATAAGTCAGAGGGTAAGTCTGAA \\
\hline
\end{tabular}




\begin{tabular}{|c|c|}
\hline 6hb_2gap2_B13 & AAGCCTTAACGTCAAAATATTAAACCAAGTACGTCATTCC \\
\hline 6hb_2gap2_B14 & GAGTCAGACTACAAATATATTTTAGTTGTAAAACCTTT \\
\hline 6hb_2gap2_B15 & TACCTGATATATGTAAATGAAAATCGCGCAGAGAATTGAA \\
\hline 6hb_2gap2_B16 & CATTTTTCAGGTTTAACAACAACTAATAGATCATATCTTT \\
\hline 6hb_2gap2_B17 & GTTTTGAGAATGGGGTGAGAAAGGCCGCAACTACTTAATT \\
\hline 6hb_2gap2_B18 & GAGCTGTGCTTTAACCTGTCGTGCCACTCATGGTTAACCG \\
\hline 6hb_2gap2_B19 & AGCCCGAAAAATCCTTTCACCAGTGAGACGAGAAACCATC \\
\hline 6hb_2gap2_B20 & GCTCATTTTACCTTTATTCAACCGTTCTGAGGGGGACTAA \\
\hline 6hb_2gap2_B21 & АСТTTATTTTCTAAAAGCCCCAAAAATAAAGGCTATCGGT \\
\hline 6hb_2gap2_B22 & GCCCCCTAACAGTGTGTTAAATCAGCTCGATAGCAGTCGA \\
\hline 6hb_2gap2_B23 & CCAGAAAGTAAGTGTAGATGGGCGCAATATTGAAACATAT \\
\hline 6hb_2gap2_B24 & ACTTGCGGCGAGGCAGCTTTCCGGCACCGAATTAATACAA \\
\hline 6hb_2gap2_B25 & TGAATAACAGTACCCAGTCACGACGTTAATTTCATCATAG \\
\hline 6hb_2gap2_B26 & CAAAGAATGAGTAATCGAATTCGTAATCGATTGCTCCTAC \\
\hline 6hb_2gap2_B27 & GGTGGCGATCGGCCTTGCTGGTAATAGCGTATTGCTTAAT \\
\hline 6hb_2gap2_B28 & AGTGAATCATAACCCTCCATTACCCAAATCAGCACAAGAA \\
\hline 6hb_2gap2_B29 & TCAGGTCTTGTTTACCAGACGACGATAAATCTACAGAACG \\
\hline 6hb_2gap2_B30 & GCATTCCATGACAACAACCATCGCCCTATTTTGTCATAGT \\
\hline 6hb_2gap2_B31 & GAACGGCCACCAATAGCCCGGAATAGATTAGCGCATGAAA \\
\hline 6hb_2gap2_B32 & CCAGCTTATCACCCTCAGAACCGCCACCTGGCCTTTTTGA \\
\hline 6hb_2gap2_B33 & CGCACCAATGGTTTACCAGCGCCAAATCGGCCTAAGAGCA \\
\hline 6hb_2gap2_B34 & TTGGGAAGGATGAAAATAGCAGCCTTTAAGGCTGCCCGCG \\
\hline 6hb_2gap2_B35 & CGGGTACCTGATGCAAATCCAATCGCGACTCTAAGAAAAC \\
\hline 6hb_2gap2_B36 & CCTGTGAAACAAGCACGTAAAACAGATTGTTTGTATTCCT \\
\hline 6hb_2gap2_B37 & TACGAGCCTGTCAGATGAATATACAGTAAATTCCAACAAT \\
\hline 6hb_2gap2_B38 & GCCCTGGGGAAATTGGGGTCGAGGTGCGAAAAAAACAAGA \\
\hline 6hb_2gap_C01 & TCAGACCATCAATGGTAATAAGTTTTTCTGAAAGGGTT \\
\hline 6hb_2gap_C02 & TCAACTTTGAGGAGCAATAGCTATCTAATAACGGCAAA \\
\hline 6hb_2gap_C03 & AAAACGCAAAGCTCAGATATAGAAGGCAAGATTACGAG \\
\hline 6hb_2gap_C04 & AGAAAAAAGGGGGTATCATATGCGTTCAACATGACAAA \\
\hline 6hb_2gap_C05 & TGAATTTAAAGCGAACCAGACCGGAACTTAGAGAAGTA \\
\hline 6hb_2gap_C06 & GATACTTAGGAATCAGGACGTTGGGATTCAACTAAATT \\
\hline 6hb_2gap_C07 & CTTAGTCGAAATTAAAACACTCATCTAAAATACGAATC \\
\hline 6hb_2gap_C08 & AGAGCAGAGCCAAGACTGTAGCGCGTGAAACCAAACCC \\
\hline 6hb_2gap_C09 & CTGAGAATAGTGCTTCTGTAAATCGTTGAATTACGACG \\
\hline 6hb_2gap_C10 & TGAGATTAACACGCGCGAACTGATAGCCTGAAAGCCTA \\
\hline 6hb_2gap_C11 & TCAAATTATAGTTTAAATGCAATGCCTTCCCAATGCTC \\
\hline 6hb_2gap_C12 & TCGGCGATAGGGCACTACGTGTAGGGCGCTGGCAAAGA \\
\hline 6hb_2gap_C13 & TGAGTGCCTATTGGATAAGTGTGAGTTTCGTCAAGTTA \\
\hline 6hb_2gap_C14 & TACGTCTTTAATCGCCTGCAATAGAGCCGTCAAGAATG \\
\hline 6hb_2gap_C15 & CCCTCAGATTTAAAAGGTGGCATCAATTCTAATTTCTG \\
\hline 6hb_2gap2_C01 & CGAGTATATATTCAGAAGCAAAAAAACATTATGTTTTT \\
\hline 6hb_2gap2_C02 & GAAGTTTCAAAAACCATAAATCAAAAAGACTTCCCAACAG \\
\hline 6hb_2gap2_C03 & GCTGAACTCACCACCAGCAGAAGATAAATAAAGCAGCAAA \\
\hline 6hb_2gap2_C04 & CAACACTATAACAACATTATTACAGGTAAGGCATAAATAG \\
\hline 6hb_2gap2_C05 & CCGAACTCAAAGTACAACGGAGATTTGTCAATCATCCAGG \\
\hline 6hb_2gap2_C06 & TCAGGAGACCCTCAAGAGAAGGATTAGGGTGTATCCCGCC \\
\hline 6hb_2gap2_C07 & CCCTCAGATTAGCGTTTGCCATCTTTTCCCTCAGATTGAC \\
\hline 6hb_2gap2_C08 & TTTTTGTGTTTTTATCCTGAATCTTACCAAATAAGAATAA \\
\hline 6hb_2gap2_C09 & TGATATTAGAGAAGAGGAAGCCCGAAATCAGGTTGTGT \\
\hline 6hb_2gap2_C10 & ATAGATATACAGTAATAAGAGAATATAACCTGTTTCGAGC \\
\hline 6hb_2gap2_C11 & GTAGATTGATTCATCAATATAATCCTGAAATAAAGCTTTT \\
\hline 6hb_2gap2_C12 & CAGTAGCCAGCAATTGAGGAAGGTTACTGGGGTGCGTA \\
\hline 6hb_2gap2_C13 & TTGTGCCGGAACCCTTCATCAAGAGTAACAAGAGTAAT \\
\hline 6hb_2gap2_C14 & TCAGTTATAAGTCCCTCATTTTCAGGATTTTTTTCAGT \\
\hline 6hb_2gap2_C15 & GAACCCGCCACCTCAGACGATTGGCCGAGTAACTCGAT \\
\hline
\end{tabular}




\begin{tabular}{|c|c|}
\hline 6hb_2gap2_C16 & TTTCCCCATATTCGCATTAGACGGGAGCTTCTGGGTAT \\
\hline 6hb_2gap2_C17 & AAAGTGTTCAGCATAATCGGCTGTCTTCGCTATTTCTT \\
\hline 6hb_2gap2_C18 & TCTGAATTATTTTAACGGATTCGCCTATGGTCATAATT \\
\hline 6hb_2gap2_C19 & GTTTTGAGAATGGGGTGAGAAAGGCCGCAACTACTTAA \\
\hline 6hb_2gap2_C20 & GAGCTGTGCTTTAACCTGTCGTGCCACTCATGGTTAAC \\
\hline 6hb_2gap2_C21 & CCCGAAAAATCCTTTCACCAGTGAGACGAGAAACCATC \\
\hline 6hb_2gap2_C22 & TCATTTTACCTTTATTCAACCGTTCTGAGGGGGACTAATG \\
\hline 6hb_2gap2_C23 & AAAAGAAGGCACGAGAGTCTGGAGCAAATCTTGTCCAT \\
\hline 6hb_2gap2_C24 & ACTTTATTTTCTAAAAGCCCCAAAAATAAAGGCTATCG \\
\hline 6hb_2gap2_C25 & CCCCTAACAGTGTGTTAAATCAGCTCGATAGCAGTCGA \\
\hline 6hb_2gap2_C26 & GCCTTGTAATCACATTAAATGTGAGCTTGATATGCCTC \\
\hline 6hb_2gap2_C27 & CCAGAAAGTAAGTGTAGATGGGCGCAATATTGAAACAT \\
\hline 6hb_2gap2_C28 & TTGCGGCGAGGCAGCTTTCCGGCACCGAATTAATACAA \\
\hline 6hb_2gap2_C29 & TTGAGAAGCCAACGGTGCGGGCCTCTTTCCTTAACAAT \\
\hline 6hb_2gap2_C30 & TGAATAACAGTACCCAGTCACGACGTTAATTTCATCAT \\
\hline 6hb_2gap2_C31 & AAGAATGAGTAATCGAATTCGTAATCGATTGCTCCTAC \\
\hline 6hb_2gap2_C32 & TTAGTGGCACAGATAAAGTGTAAAGCTCTAAAAGTGCC \\
\hline 6hb_2gap2_C33 & CTGACTAAAGATTAGTACCTTTACTAATAGTAGTACGGAT \\
\hline 6hb_2gap2_C34 & GTGAGGAAAGGAGCAAATGAAAAATCACAGAGGACATCGC \\
\hline 6hb_2gap2_C35 & GGCCAGCCATTGCTTTGATTAGTAATGTGAGGCGACAGGA \\
\hline 6hb_2gap2_C36 & AACGGTGGCTGAGAGGCGCAGACGGTATCATCGCCAGCGA \\
\hline 6hb_2gap2_C37 & GCAAATCGCTGATCGAGGTGAATTTCAATCTCCAGGAACA \\
\hline 6hb_2gap2_C38 & GATTCAGGCAGGCTCAGAACCGCCACATAATCAGGCATTT \\
\hline 6hb_2gap2_C39 & GCCAGCGATTGAGACACCACGGAATATATGTTAGAATACC \\
\hline 6hb_2gap2_C40 & TGGCGCCAATCATAATGCAGAACGCGAGTACCGTAATTTA \\
\hline 6hb_2gap2_C41 & GTGCCCTTTTTCCTTTTTAACCTCCGATTAAGAAATTAAT \\
\hline 6hb_2gap_D01 & GGTTGTCCGTGGGAAACGTCACCAATTTTCATCAAATC \\
\hline 6hb_2gap_D02 & CGGGAGTGAAATAATCCTTTGCCCGAATCATCAGATTA \\
\hline 6hb_2gap_D03 & GTAGCGTCTGTCAGGCCGATTAAAGGGCTTTGATAATG \\
\hline 6hb_2gap_D04 & ATCAGAAAAGGATTCAGCGGAGTGAGTTTCCAGATTGT \\
\hline 6hb_2gap_D05 & GGCCAAATACCGAACGAACCAGTCACACGACAATTACA \\
\hline 6hb_2gap_D06 & GTGAAATACCAGTACCACATTCCAATACTGCGGAGAAC \\
\hline 6hb_2gap_D07 & CTACGAATACACCCGCGACCTACGTAACAAAGCGAAAG \\
\hline 6hb_2gap_D08 & CATGAAAGCGCGAAACAACGGCTACAGAGGCTTCGGAA \\
\hline 6hb_2gap_D09 & GCACCTAGCGTCCCACCGGAAGAATGGAAAGCGATCAA \\
\hline 6hb_2gap_D10 & AGAACGGAGGTTAATTTGCCATTGAGCGCTAATCCTCC \\
\hline 6hb_2gap_D11 & GTATAAATCGCCTCCAGACGACCGCACTCATCGAGGGC \\
\hline 6hb_2gap_D12 & AAAGCGCATTTTCGAGCAATAAGAATAAACAATGATAA \\
\hline 6hb_2gap_D13 & AAGTTACCACCAAAGGGTTAGGCGAATTATTCATGCGG \\
\hline 6hb_2gap2_D01 & GAAGTTTCAAAAACCATAAATCAAAAAGACTTCCCAAC \\
\hline 6hb_2gap2_D02 & GTAGACGAGAAGTGTTTTTATAATCAAACATCACAATATT \\
\hline 6hb_2gap2_D03 & ACACTATAACAACATTATTACAGGTAAGGCATAAATAG \\
\hline 6hb_2gap2_D04 & CGATAAAATAATTTTTTCACGTTGAATTAAACAACCGATA \\
\hline 6hb_2gap2_D05 & AGGAGACCCTCAAGAGAAGGATTAGGGTGTATCCCGCC \\
\hline 6hb_2gap2_D06 & ATCAACCAAGACTCCTTATTACGCAGAGTTTATGGGCGAC \\
\hline 6hb_2gap2_D07 & TTTGTGTTTTTATCCTGAATCTTACCAAATAAGAATAA \\
\hline 6hb_2gap2_D08 & TGATATTAGAGAAGAGGAAGCCCGAAATCAGGTTGTGTAG \\
\hline 6hb_2gap2_D09 & ATAACGCAAACATAGCGATAGCTTAGGCTTAGGAGAACGC \\
\hline 6hb_2gap2_D10 & AGATTGATTCATCAATATAATCCTGAAATAAAGCTTTT \\
\hline 6hb_2gap2_D11 & CAGATCTCAAATATCAAACGCTCAATCGTCTAAAAATA \\
\hline 6hb_2gap2_D12 & ATAAATAATAATGCTGTAGAGACTGGATAGCGTCATAATA \\
\hline 6hb_2gap2_D13 & GAGATATAACGCAAGAAGTTTTGCCAAGCTGATTTAAT \\
\hline 6hb_2gap2_D14 & $\begin{array}{l}\text { GGTAGGACCAACTTTGATCACCCTCAGCAGCTGCGCTT } \\
\end{array}$ \\
\hline 6hb_2gap2_D15 & TGGGAAGCCGCCACCAGAAGGTGAATTATCAGCCGGAA \\
\hline 6hb_2gap2_D16 & GGCGTAGTCCTGAACAATTAATGGTTTGAAAAAATCTT \\
\hline 6hb_2gap2_D17 & TACATTTTTCAGGTTTAACAACAACTAATAGATCATATCT \\
\hline 6hb_2gap2_D18 & GCGTTTTGAGAATGGGGTGAGAAAGGCCGCAACTACTTAA \\
\hline 6hb_2gap2_D19 & GGGAGCTGTGCTTTAACCTGTCGTGCCACTCATGGTTAAC \\
\hline 6hb_2gap2_D20 & CCCGAAAAATCCTTTCACCAGTGAGACGAGAAACCATCAC \\
\hline
\end{tabular}




\begin{tabular}{|c|c|}
\hline 6hb_2gap2_D21 & САACTTTATTTTCTAAAAGCCCCAAAAATAAAGGCTATCG \\
\hline 6hb_2gap2_D22 & CCCCTAACAGTGTGTTAAATCAGCTCGATAGCAGTCGAGA \\
\hline 6hb_2gap2_D23 & TACCAGAAAGTAAGTGTAGATGGGCGCAATATTGAAACAT \\
\hline 6hb_2gap2_D24 & TTGCGGCGAGGCAGCTTTCCGGCACCGAATTAATACAAAA \\
\hline 6hb_2gap2_D25 & $\begin{array}{l}\text { AGTGAATAACAGTACCCAGTCACGACGTTAATTTCATCAT } \\
\end{array}$ \\
\hline 6hb_2gap2_D26 & AAGAATGAGTAATCGAATTCGTAATCGATTGCTCCTACCA \\
\hline 6hb_2gap2_D27 & GACTAAAGATTAGTACCTTTACTAATAGTAGTACGGAT \\
\hline 6hb_2gap2_D28 & TTCCATGACCCTCAATCAATATCTGGGCGCTCAGGACA \\
\hline 6hb_2gap2_D29 & GGCCAGCCATTGCTTTGATTAGTAATGTGAGGCGACAG \\
\hline 6hb_2gap2_D30 & ССАТCTTCTCAACATGTTTTAAATATGGAGACAACAGTTC \\
\hline 6hb_2gap2_D31 & TCAGTGAATCATAACCCTCCATTACCCAAATCAGCACAAG \\
\hline 6hb_2gap2_D32 & GTTGACGAAGAGGACAGATGAACGGTAATCATAAGACT \\
\hline 6hb_2gap2_D33 & GCAAATCGCTGATCGAGGTGAATTTCAATCTCCAGGAA \\
\hline 6hb_2gap2_D34 & TCGCATTCCATGACAACAACCATCGCCCTATTTTGTCATA \\
\hline 6hb_2gap2_D35 & $\begin{array}{l}\text { AGGAACGGCCACCAATAGCCCGGAATAGATTAGCGCATGA } \\
\end{array}$ \\
\hline 6hb_2gap2_D36 & AGGTCTAAACCACCACCAGAGCCGCCTTGACCGCCATT \\
\hline 6hb_2gap2_D37 & GCCAGCGATTGAGACACCACGGAATATATGTTAGAATA \\
\hline 6hb_2gap2_D38 & AAACCAGAGGGAAGATTTATCCCAATCCAACGCTAAGTTG \\
\hline 6hb_2gap2_D39 & GGGAAGGATGAAAATAGCAGCCTTTAAGGCTGCCCGCGCC \\
\hline 6hb_2gap2_D40 & CGCCAATGAAAAATAATATCCCATCCCGATTAATTACT \\
\hline 6hb_2gap2_D41 & GTGCCCTTTTTCCTTTTTAACCTCCGATTAAGAAATTA \\
\hline 6hb_2gap2_D42 & TTCCTGTGAAACAAGCACGTAAAACAGATTGTTTGTATTC \\
\hline 6hb_2gap_E01 & CAGTTCTAACTCTAGAACCCTTCTGACCCTAAATGAGG \\
\hline 6hb_2gap_E02 & CGCCAACGCGCGCGCGTACTATGGTTGATTTTACACCG \\
\hline 6hb_2gap_E03 & AGGCTGAGGGTATGAGATGGTTTAATAGAAAAATCATC \\
\hline 6hb_2gap_E04 & TAGGCTAATCGTTCCATTAAACGGGTTTGACCCCCTGA \\
\hline 6hb_2gap_E05 & GGAACAAATTTTCCCGTATAACACAGACAGCCCTTAAA \\
\hline 6hb_2gap_E06 & AAGTTACCGAGCCATTATCATAAACAAACATCAGAGGA \\
\hline 6hb_2gap_E07 & AACAGAGAGCCTTTGAAGCCTTAAATCTTATCCGTGCC \\
\hline 6hb_2gap_E08 & AACATAATTCTGATATTTAACAACGCATACAAATACGC \\
\hline 6hb_2gap_E09 & CAGGAAGAGGTCCATATAACAGTTGATGAGTAACTTTA \\
\hline 6hb_2gap_E10 & ATTAAAGTGTACAAATAATTCGCGTCCTCAGAAACCGT \\
\hline 6hb_2gap_E11 & AAAGAATGAAATGGACGACGACAGTAGACAAAATTTGT \\
\hline 6hb_2gap_E12 & TTAGCTAGCCCCCTTATTAGAGCCAGCAAAAGATGAGC \\
\hline 6hb_2gap_E13 & CAACTAGATGATGGCAAGGATTTAGAAGTATTTTAGAT \\
\hline 6hb_2gap2_E01 & GTAGACGAGAAGTGTTTTTATAATCAAACATCACAATA \\
\hline 6hb_2gap2_E02 & CGATAAAATAATTTTTTCACGTTGAATTAAACAACCGA \\
\hline 6hb_2gap2_E03 & ATCAACCAAGACTCCTTATTACGCAGAGTTTATGGGCG \\
\hline 6hb_2gap2_E04 & ATAACGCAAACATAGCGATAGCTTAGGCTTAGGAGAAC \\
\hline 6hb_2gap2_E05 & TCAGGGCAAGTTTTGCCGGCGAACGTGGCGGGCAAGTTCC \\
\hline 6hb_2gap2_E06 & TCATTTTACCTTTATTCAACCGTTCTGAGGGGGACTAAT \\
\hline 6hb_2gap2_E07 & TTCCATGACCCTCAATCAATATCTGGGCGCTCAGGACA \\
\hline 6hb_2gap2_E08 & CCGGATTTGCAACAAAAGGAATTACGGAAAGATTCTAC \\
\hline 6hb_2gap2_E09 & GAACGGCCACCAATAGCCCGGAATAGATTAGCGCATGA \\
\hline 6hb_2gap2_E10 & ACCAGAGGGAAGATTTATCCCAATCCAACGCTAAGTTG \\
\hline 6hb_2gap2_E11 & CCTGTGAAACAAGCACGTAAAACAGATTGTTTGTATTC \\
\hline 6hb_2gap_F01 & CATTTGTCGGGACCTCGTTAGCAGTAATAAAAGCTGCC \\
\hline 6hb_2gap_F02 & AATGTAATATGAATGCGATTTCAAATGCTTTAAGTCAA \\
\hline 6hb_2gap_F03 & $\begin{array}{l}\text { GGGATTAATCAGGTATGGGATTTTGAGGACTAATGTAC } \\
\end{array}$ \\
\hline 6hb_2gap_F04 & TTCATACGTTGGCAGATAGCCTCACCAGTAGCATAATG \\
\hline 6hb_2gap_F05 & CCTAAGGGTTTTCATAAATCACCGGAATCATAAGTTGG \\
\hline 6hb_2gap_F06 & GTTGAACCAGGCTCGGAACCTATTATAACGGGGAACCA \\
\hline 6hb_2gap_F07 & AAGAATACATACCGAGGAAACGCAATTACCGAACGTGC \\
\hline 6hb_2gap_F08 & GGTACTACAGGGGGGAGAGGCGGTTTTCCAGAACTTGC \\
\hline 6hb_2gap_F09 & ATAAATTGGGCTGCTATTTTTGAGAGAAACCAAGTAAG \\
\hline 6hb_2gap_F10 & TAAAGACGATCTATTGTAAACGTTAAACGCATAGCTTG \\
\hline 6hb_2gap_F11 & TTTGCAAGCAAAGCCATTCGCCATTCCAGAGAGAAACG \\
\hline
\end{tabular}




\begin{tabular}{|c|c|}
\hline 6hb_2gap_F12 & TCССTATTACATCATGCCTGCAGGTCAAGACAATTGGG \\
\hline 6hb_2gap_F13 & TTATCCGTATTATGTTATCCGCTCACACAGTACAAATT \\
\hline 6hb_2gap2_F01 & TGAACTCACCACCAGCAGAAGATAAATAAAGCAGCAAA \\
\hline 6hb_2gap2_F02 & GAACTCAAAGTACAACGGAGATTTGTCAATCATCCAGG \\
\hline 6hb_2gap2_F03 & CTCAGATTAGCGTTTGCCATCTTTTCCCTCAGATTGAC \\
\hline 6hb_2gap2_F04 & AGATATACAGTAATAAGAGAATATAACCTGTTTCGAGC \\
\hline 6hb_2gap2_F05 & GGTCAAGAACTCAAACTAAAGGAGCGGGCGCAAGGAAG \\
\hline 6hb_2gap2_F06 & AGTGAATCATAACCCTCCATTACCCAAATCAGCACAAG \\
\hline 6hb_2gap2_F07 & ACTACGTTGCGCCGACAATGTACCGTAACACCCAGCCC \\
\hline 6hb_2gap2_F08 & AATTTGTTTAGTACCGCCCTCATTAAAGCCACCTCACA \\
\hline 6hb_2gap2_F09 & ATAACTAGAAAATTCATAAGTCAGAGGGTAAGTCTGAA \\
\hline 6hb_2gap2_F10 & AAGCCTTAACGTCAAAATATTAAACCAAGTACGTCATT \\
\hline 6hb_2gap2_F11 & CCTGATATATGTAAATGAAAATCGCGCAGAGAATTGAA \\
\hline 6hb_2gap2_F12 & CATTTTTCAGGTTTAACAACAACTAATAGATCATATCT \\
\hline 6hb_2gap2_F13 & $\begin{array}{l}\text { GTGAGGAAAGGAGCAAATGAAAAATCACAGAGGACATC } \\
\end{array}$ \\
\hline 6hb_2gap2_F14 & GGTGGCGATCGGCCTTGCTGGTAATAGCGTATTGCTTA \\
\hline 6hb_2gap2_F15 & CCATCTTCTCAACATGTTTTAAATATGGAGACAACAGT \\
\hline 6hb_2gap2_F16 & CCGGATTTGCAACAAAAGGAATTACGGAAAGATTCTAC \\
\hline 6hb_2gap2_F17 & AGGTCTTGTTTACCAGACGACGATAAATCTACAGAACG \\
\hline 6hb_2gap2_F18 & AACGGTGGCTGAGAGGCGCAGACGGTATCATCGCCAGC \\
\hline 6hb_2gap2_F19 & GCATTCCATGACAACAACCATCGCCCTATTTTGTCATA \\
\hline 6hb_2gap2_F20 & AGCTTATCACCCTCAGAACCGCCACCTGGCCTTTTTGA \\
\hline 6hb_2gap2_F21 & GATTCAGGCAGGCTCAGAACCGCCACATAATCAGGCAT \\
\hline 6hb_2gap2_F22 & CGCACCAATGGTTTACCAGCGCCAAATCGGCCTAAGAG \\
\hline 6hb_2gap2_F23 & GGGAAGGATGAAAATAGCAGCCTTTAAGGCTGCCCGCG \\
\hline 6hb_2gap2_F24 & TGGCGCCAATCATAATGCAGAACGCGAGTACCGTAATT \\
\hline 6hb_2gap2_F25 & CGGGTACCTGATGCAAATCCAATCGCGACTCTAAGAAA \\
\hline 6hb_2gap2_F26 & CGAGCCTGTCAGATGAATATACAGTAAATTCCAACAAT \\
\hline 6hb_3gap_A01 & GATAATTGCCTCAACCTAAACGAGAAACACCAAAGG \\
\hline 6hb_3gap_A02 & ATAATCATCAAGTAGCGACATCATACATGGCTCCTG \\
\hline 6hb_3gap_A03 & AACGGGGCGATCGCTCAACATAGGAATCATTAGCAA \\
\hline 6hb_3gap_A04 & AGCACGGAAGCACAATATTTTAGACTTTACAACACA \\
\hline 6hb_3gap_A05 & TAAAACAGAGAACATTAATTGCGTTTCAGTTGTCAC \\
\hline 6hb_3gap_A06 & TACCGGAAGTTAAAACTAGCATGTCGTACAGAAAGG \\
\hline 6hb_3gap_A07 & GTCAAAGGCCGGAACAAACGGCGGAGCCAGCAGCCA \\
\hline 6hb_3gap_A08 & AGAGCTGTTTAGATGTGCTGCAAGGTAATTTAATCA \\
\hline 6hb_3gap_A09 & AAACAATTCGAGCTTCCATTGAATCCCCCTTAAATC \\
\hline 6hb_3gap_A10 & CCGCGCCAGAATCCTGCTGCGCGTAACCACCAAAGT \\
\hline 6hb_3gap_A11 & CGTAGAATTGCGAATACGCCTGTAGCATTCACCCAG \\
\hline 6hb_3gap_A12 & AACAACTGGCATGATTACAAGAATTGAGTTAAATCA \\
\hline 6hb_3gap_A13 & ATTATAGAATCCTTGAAAAAGAAGATGATGTTTTTC \\
\hline 6hb_3gap_B01 & AAAGTTTTTCTCTTATAAATCACACCCGCCGCGGGC \\
\hline 6hb_3gap_B02 & TCGGTATTTAAAAAGTTTTGTCGTCAATAGAAAAAA \\
\hline 6hb_3gap_B03 & TGAATCCAGCCGTTTTAGCGAAGCCCAATAATCAGG \\
\hline 6hb_3gap_B04 & AAAAAAGCTTGTTAACAATTTCATTCGCTATTCGCT \\
\hline 6hb_3gap_B05 & AATCGATGGCCTTGAGTGTTGTTCCGATGGTGCAGC \\
\hline 6hb_3gap_B06 & CAAAATAATGGGAAGGAGCGGAATTACGTTATTAGC \\
\hline 6hb_3gap_B07 & TAACAAACAGGCATCACGCAGAAATGGATTATTCAG \\
\hline 6hb_3gap_B08 & TAAAACGAACTAACGGAAGGCTTGCCCTGAACTGCT \\
\hline 6hb_3gap_B09 & ATGACAACAGTGCCTTTAATGAAAGACAGCATTGCT \\
\hline 6hb_3gap_B10 & CAGGGAGGCTGAGACTCGTTCCAGTAAGCGGACAGT \\
\hline 6hb_3gap_B11 & AGAAAGGAAACATAAAGGTGCCGTCACCGACTACAA \\
\hline 6hb_3gap_B12 & TAGCACCCAGCTACAATTTTATTTTCATCGGTAGAA \\
\hline 6hb_3gap_B13 & TGGAAACCTTGAATTTATCATACCGACCGTGTATAT \\
\hline 6hb_3gap_B14 & AAGATTCATTCATTTTTGCGGATGGGCAAACTAAAT \\
\hline 6hb_3gap_C01 & CAGACCATCAATGGTAATAAGTTTTTCTGAAAGGGT \\
\hline 6hb_3gap_C02 & CAACTTTGAGGAGCAATAGCTATCTAATAACGGCAA \\
\hline 6hb_3gap_C03 & AAACGCAAAGCTCAGATATAGAAGGCAAGATTACGA \\
\hline
\end{tabular}




\begin{tabular}{|c|c|}
\hline 6hb_3gap_C04 & GAAAAAAGGGGGTATCATATGCGTTCAACATGACAA \\
\hline 6hb_3gap_C05 & GAATTTAAAGCGAACCAGACCGGAACTTAGAGAAGT \\
\hline 6hb_3gap_C06 & ATACTTAGGAATCAGGACGTTGGGATTCAACTAAAT \\
\hline 6hb_3gap_C07 & TTAGTCGAAATTAAAACACTCATCTAAAATACGAAT \\
\hline 6hb_3gap_C08 & GAGCAGAGCCAAGACTGTAGCGCGTGAAACCAAACC \\
\hline 6hb_3gap_C09 & TGAGAATAGTGCTTCTGTAAATCGTTGAATTACGAC \\
\hline 6hb_3gap_C10 & GAGATTAACACGCGCGAACTGATAGCCTGAAAGCCT \\
\hline 6hb_3gap_C11 & CAAATTATAGTTTAAATGCAATGCCTTCCCAATGCT \\
\hline 6hb_3gap_C12 & CGGCGATAGGGCACTACGTGTAGGGCGCTGGCAAAG \\
\hline 6hb_3gap_C13 & GAGTGCCTATTGGATAAGTGTGAGTTTCGTCAAGTT \\
\hline 6hb_3gap_C14 & ACGTCTTTAATCGCCTGCAATAGAGCCGTCAAGAAT \\
\hline 6hb_3gap_C15 & CCTCAGATTTAAAAGGTGGCATCAATTCTAATTTCT \\
\hline 6hb_3gap_D01 & GTTGTCCGTGGGAAACGTCACCAATTTTCATCAAAT \\
\hline 6hb_3gap_D02 & GGGAGTGAAATAATCCTTTGCCCGAATCATCAGATT \\
\hline 6hb_3gap_D03 & TAGCGTCTGTCAGGCCGATTAAAGGGCTTTGATAAT \\
\hline 6hb_3gap_D04 & TCAGAAAAGGATTCAGCGGAGTGAGTTTCCAGATTG \\
\hline 6hb_3gap_D05 & GCCAAATACCGAACGAACCAGTCACACGACAATTAC \\
\hline 6hb_3gap_D06 & TGAAATACCAGTACCACATTCCAATACTGCGGAGAA \\
\hline 6hb_3gap_D07 & TACGAATACACCCGCGACCTACGTAACAAAGCGAAA \\
\hline 6hb_3gap_D08 & ATGAAAGCGCGAAACAACGGCTACAGAGGCTTCGGA \\
\hline 6hb_3gap_D09 & CACCTAGCGTCCCACCGGAAGAATGGAAAGCGATCA \\
\hline 6hb_3gap_D10 & GAACGGAGGTTAATTTGCCATTGAGCGCTAATCCTC \\
\hline 6hb_3gap_D11 & TATAAATCGCCTCCAGACGACCGCACTCATCGAGGG \\
\hline 6hb_3gap_D12 & AAGCGCATTTTCGAGCAATAAGAATAAACAATGATA \\
\hline 6hb_3gap_D13 & AGTTACCACCAAAGGGTTAGGCGAATTATTCATGCG \\
\hline 6hb_3gap_E01 & AGTTCTAACTCTAGAACCCTTCTGACCCTAAATGAG \\
\hline 6hb_3gap_E02 & GCCAACGCGCGCGCGTACTATGGTTGATTTTACACC \\
\hline 6hb_3gap_E03 & GGCTGAGGGTATGAGATGGTTTAATAGAAAAATCAT \\
\hline 6hb_3gap_E04 & AGGCTAATCGTTCCATTAAACGGGTTTGACCCCCTG \\
\hline 6hb_3gap_E05 & GAACAAATTTTCCCGTATAACACAGACAGCCCTTAA \\
\hline 6hb_3gap_E06 & AGTTACCGAGCCATTATCATAAACAAACATCAGAGG \\
\hline 6hb_3gap_E07 & ACAGAGAGCCTTTGAAGCCTTAAATCTTATCCGTGC \\
\hline 6hb_3gap_E08 & ACATAATTCTGATATTTAACAACGCATACAAATACG \\
\hline 6hb_3gap_E09 & AGGAAGAGGTCCATATAACAGTTGATGAGTAACTTT \\
\hline 6hb_3gap_E10 & TTAAAGTGTACAAATAATTCGCGTCCTCAGAAACCG \\
\hline 6hb_3gap_E11 & AAGAATGAAATGGACGACGACAGTAGACAAAATTTG \\
\hline 6hb_3gap_E12 & TAGCTAGCCCCCTTATTAGAGCCAGCAAAAGATGAG \\
\hline 6hb_3gap_E13 & AACTAGATGATGGCAAGGATTTAGAAGTATTTTAGA \\
\hline 6hb_3gap_F01 & ATTTGTCGGGACCTCGTTAGCAGTAATAAAAGCTGC \\
\hline 6hb_3gap_F02 & ATGTAATATGAATGCGATTTCAAATGCTTTAAGTCA \\
\hline 6hb_3gap_F03 & GGATTAATCAGGTATGGGATTTTGAGGACTAATGTA \\
\hline 6hb_3gap_F04 & TCATACGTTGGCAGATAGCCTCACCAGTAGCATAAT \\
\hline 6hb_3gap_F05 & CTAAGGGTTTTCATAAATCACCGGAATCATAAGTTG \\
\hline 6hb_3gap_F06 & TTGAACCAGGCTCGGAACCTATTATAACGGGGAACC \\
\hline 6hb_3gap_F07 & AGAATACATACCGAGGAAACGCAATTACCGAACGTG \\
\hline 6hb_3gap_F08 & GTACTACAGGGGGGAGAGGCGGTTTTCCAGAACTTG \\
\hline 6hb_3gap_F09 & TAAATTGGGCTGCTATTTTTGAGAGAAACCAAGTAA \\
\hline 6hb_3gap_F10 & AAAGACGATCTATTGTAAACGTTAAACGCATAGCTT \\
\hline 6hb_3gap_F11 & TTGCAAGCAAAGCCATTCGCCATTCCAGAGAGAAAC \\
\hline 6hb_3gap_F12 & СССТATTACATCATGCCTGCAGGTCAAGACAATTGG \\
\hline 6hb_3gap_F13 & TATCCGTATTATGTTATCCGCTCACACAGTACAAAT \\
\hline
\end{tabular}

\section{Supplementary Table 6. Staple list of 6HB designs.}




\begin{tabular}{|c|c|c|}
\hline \multicolumn{3}{|c|}{ 10HB design } \\
\hline Name & Sequence $\left(5^{\prime} \rightarrow 3^{\prime}\right)$ & Color \\
\hline 10hb_001 & AAGCTGAGAATAGAAATATGTACCCCAATAGCGACGATAAAAA & \\
\hline 10hb_002 & AAACCGGCGGATTGACCCAGAAGGATTTGCCAAAGGGAAAGTG & \\
\hline 10hb_003 & TTTTATTACAATAATTGAATACCAAGTTTCGCTATTAG & \\
\hline 10hb_004 & CGTCACCGCAGTTCCAGACCAGGCGGGATACCGATAGTGGCA & \\
\hline 10hb_005 & AATATTCAATGGCAAGGTAGCTGATATGCCGGAAACCAGAAT & \\
\hline 10hb_006 & CTATGGTTAAATACCGAGATAATACAATTTAACAATTTTCCG & \\
\hline 10hb_007 & CATCAGACAAAAGTGCCCGAACCTATTATTCTCCCTCAGTTTC & \\
\hline 10hb_008 & CACCAATATTGGTACTGGCTCCTCAAGAGAAGGGAATAGACTG & \\
\hline 10hb_009 & TTGGTTTAGGACGGGTAACATCGCCCACGCATGTATCGGTGTC & \\
\hline 10hb_010 & TTATAAAGGAATTTGAGGCAGGGAGTTAAAGGTGAAAATATTT & \\
\hline 10hb_011 & GATTAAGATTGGGCTTGCTCGTTTAAAAGACAGCATCGTGTC & \\
\hline 10hb_012 & GTCATTTTGCAGTAATACCTGAGTAATGTGTAAAGAGAACAGG & \\
\hline 10hb_013 & TGACTTTAGACAGCATAACGGAGACAGTCAAATACAAAGTATT & \\
\hline 10hb_014 & GGACTTTGGGGCTCTAGACAGGCTGCGCAACTGCCTCAGTGGC & \\
\hline 10hb_015 & TGTTAGGGAGCTAAAACGTTCGCTATTACGCCTAACCGTGAGT & \\
\hline 10hb_016 & TTTTTATAGGCAAAATCCGTGGCGTCTAAGTTGGGTAATCAC & \\
\hline 10hb_017 & GGAATAGGGCGCCAGCAGAAATCAACAGTTGATAAAAGTCAGA & \\
\hline 10hb_018 & AGCGCACACCCCGGTCAGTCTTTAGGAGCACTCGACAACTATA & \\
\hline 10hb_019 & GAACAACCAATATAGGTCTGGAAACAGTACATGCAAAAGTTTC & \\
\hline 10hb_020 & CTGTCGGGTATATTAAGAGCTTCTGTAAATCGACA & \\
\hline 10hb_021 & AAGGGCATTTTCGAGCCAAATACCGTTCTGAC & \\
\hline 10hb_022 & GTACCGACACTCATGAAAACATAATTAA & \\
\hline 10hb_023 & ATCAAGTTTCCACCAGGGAGGTTGAGGCAGGAG & \\
\hline 10hb_024 & GTAGGAGCCACGATTGGCCTTGATATTTTAACAGGGAGGAAGA & \\
\hline 10hb_025 & TAGCCCGCCTCCCAGAATGGAAAGCCATACATTGAATTAGAAA & \\
\hline 10hb_026 & AAGACAGGCGCCAAAAGAATACACTAATGCCATTCATCACATG & \\
\hline 10hb_027 & AACGACTGACCTATACCAAGCGCGAACTTTTTGATACATACCG & \\
\hline 10hb_028 & ACGATAGCCGGCGCCTGATAAATTGGAACGAGACACTATAAGA & \\
\hline 10hb_029 & TTCACCAATTCTACATTTCGCAAATGGAGAAGAAATAGCAAAC & \\
\hline 10hb_030 & CCGGAGTACGGATTTGGGGCGCGAGTCGGTTGGTAATAGATAA & \\
\hline 10hb_031 & AATTAATATAAAGTAGTAGCATTAATTAGCAAGAATCGTCTGA & \\
\hline 10hb_032 & GGGCGGGTGGTGTTTCCTGTGTGAACGGGTACCCCAAATAAAA & \\
\hline 10hb_033 & TTGCCGGCCAACATACGAGCCGGAAGTGCCAAAATCGGAGTCA & \\
\hline 10hb_034 & ATCCTTTCCAGATGAGTGAGCTAACCGCCAGGGGAAAGCTTAT & \\
\hline 10hb_035 & GAGTCAGTCACCAGAGATAGAACCCAAAATCTAAAGGAGTTTC & \\
\hline 10hb_036 & AATAGCTCAATGCACAGACAATATTACCGCCTCTGCGCGGCTA & \\
\hline 10hb_037 & AGAATTGCAACGAACTGATAGCCCTACCAGCACAGGGCGTTTT & \\
\hline 10hb_038 & AAGCATGCGTTCTATATGTAAATGCCTACCTTTACGAGCAGAA & \\
\hline 10hb_039 & TTTACACCGGAACGCGAGAAAACTTAAGAGTCTATCATTATAG & \\
\hline 10hb_040 & CTCACGCGTTTTTAGCAAGGCCGGACCGATTGGGGGTCAGAAA & \\
\hline 10hb_041 & GGAAGTTTGCCTGGGAATTAGAGCCTTAAAGGGGCTTTTATTA & \\
\hline 10hb_042 & AGACGTAATCTAATCTACGTTAATAAGAAAGACTACGAATGCG & \\
\hline 10hb_043 & CCGATAACAAATAAGAACTGGCTCATAATGCACATGAGGATAT & \\
\hline 10hb_044 & TACTGAAACACTTAATTTCAACTTTAAGAGCAGGTAGCATGCG & \\
\hline 10hb_045 & ATTCAATATCGAGCGGATTGCATCAAAAACCACCTTTATCATA & \\
\hline 10hb_046 & CTAAAAGCAAACAAAAATCAGGTCTAGAGGGGTACCAAAATTC & \\
\hline 10hb_047 & GCTGGCTCCTTCAAATGCTTTAAACTACTGCGAATTAAGCAAT & \\
\hline 10hb_048 & GCCAAACAGCTTCAAAGGGCGAAAAACCATCACGAGCTCGGCA & \\
\hline 10hb_049 & GAATAGCAAGCTTTGGAACAAGAGTAGCACTAGCTTGCAAGGG & \\
\hline 10hb_050 & CCGCTGTTTGAAATCAAAAGAATAGTTGACGGGTTTTCCGAAA & \\
\hline 10hb_051 & TCACAAAAGAGCCAGAATCCTGAGAGAAAGCGAAAGCATATCA & \\
\hline 10hb_052 & TGACCTTCTTTAACAGGAGGCCGATGGTCACGGCAACAGTGAG & \\
\hline
\end{tabular}




\begin{tabular}{|c|c|}
\hline 10hb_053 & GCCACTCAAACACGTATAACGTGCTGCCGCTAGAAGATAAATA \\
\hline 10hb_054 & TCATCAACGCTTTTATCAACAATAGCCTAATTTTTAACCCATT \\
\hline 10hb_055 & TAAAACAACGCACGACAATAAACAACTTTCCTAATAGTGTATA \\
\hline 10hb_056 & CCTCCAGGAGTACGGAAAGCAACATATAAAAGCCGTAACGTGT \\
\hline 10hb_057 & AAAGAGGATAGGCTGGCACCGGAACCAGCTGAATTTAAAACG \\
\hline 10hb_058 & СССCCATTAAAATACCACCGGAATACCCAAAAAAAGTTTTTTA \\
\hline 10hb_059 & ATTTCAGAGGCTTACGAGGAACAAAGTTACCAGTATGGGCTCC \\
\hline 10hb_060 & GCTATGACCCTAAAGAAGAAGCCCAATAATAACCAAAAATCGA \\
\hline 10hb_061 & ATTCGCCTCAGTGGATAGTTGAGCGCTAATATACGTTAAGCTA \\
\hline 10hb_062 & TCATAGCTTTTTCTTTTGCGGATGGCTTATAAATCATAATGG \\
\hline 10hb_063 & ATTCAGGTCGATCGAGGTCTTTACAGAGAGAATCGCGTCGAAG \\
\hline 10hb_064 & TGGGGACGTTGCCCCGATAGAAACGATTTTTTTGTGAGCGCAT \\
\hline 10hb_065 & AAGACTGAGAGCTGGCAATTACCAACGCTAACTGATTATTTGA \\
\hline 10hb_066 & CTTTGGTGAGGGCCGCGCTTAGTTGCTATTTTTTTGGATTCGT \\
\hline 10hb_067 & TTATATAAATACAAATTATCCAGAACAATCGCCATTAATGGG \\
\hline 10hb_068 & CAAGCAAAATCCAATAATGAAGGCTTATCCGGCGTAGATAAGA \\
\hline 10hb_069 & TTAAAGCTTAGTAAACCATAGGAATCATTACCGTACCTTCGCG \\
\hline 10hb_070 & TAACAGGGCGAATTTTGTCACAATCCCCTCATAACC \\
\hline 10hb_071 & CCAACCTACCACATTATCGCAGTATGTTAGCGTAGCATAAGT \\
\hline 10hb_072 & TCGTAATCCAGGCCCACCGGGAGAATTAACTTCAGCTCTTAA \\
\hline 10hb_073 & GCTTAGGTAAAAATAATAACCTCCCGACTTGATATCAATGAG \\
\hline 10hb_074 & GCCACCCTCATATTTCGGTATAAAATTGACAAACCACC \\
\hline 10hb_075 & AAATTTACATCGGGAGAATTCATCGAGTACCGCAA \\
\hline 10hb_076 & GCCACCAAATAGAAGCGCCAAGATAGCAACAGA \\
\hline 10hb_nogap_A01 & CACCACGGAAAGCCCAAGTTTAGTACCGCCAGAAACATGTGC \\
\hline 10hb_nogap_A02 & ATACATACATCCAGTACTATAAGTATAGCCCGATTAGGGATG \\
\hline 10hb_nogap_A03 & ATTAAGACTCTCATAGTTGAATTTCTTAAACAGCTTATTCCA \\
\hline 10hb_nogap_A04 & AGGAAACGCAACGTTAGAGGAGCCTTTAATTAACCGATAAGT \\
\hline 10hb_nogap_A05 & AAAGTAAGCACTTTCAATAATTTTTTCACGTCCGCTTTACG \\
\hline 10hb_nogap_A06 & GAAATCCGTAGTTTGACCAAGGATACCAGACTATCTTACCG \\
\hline 10hb_nogap_A07 & AATGAAATAGCGGTTGAACTAGCATGTCAATGAACCCTTTCA \\
\hline 10hb_nogap_A08 & CCCACAAGAAAAGCAAAAGTCTGGAGCAAACGGTAAAGAAC \\
\hline 10hb_nogap_A09 & ACAAAGTCAGTCGCATTATTTTTGAGAGATCTCACCATCAA \\
\hline 10hb_nogap_A10 & CAGGGAAGCGAGGAACGTTTCCGGCACCGCTTCTGGAAATTT \\
\hline 10hb_nogap_A11 & AAAATGAAAACCAGCTTCGACGACAGTATCGGTTGGGATGC \\
\hline 10hb_nogap_A12 & TTATCCCAATCGGATTCAGATGGGCGCATCGAGCTGGCCAG \\
\hline 10hb_nogap_A13 & ATTAATTGGGGACATTCCTGAACCAGAAAGGGTTACAAAAT \\
\hline 10hb_nogap_A14 & CAGAGCCTAAGCGGAATCGGAACAAAGAAACACCCTCACACC \\
\hline 10hb_nogap_A15 & СAATTTTATCTCATCAAAACGTTATTAATTTAAGGAATTGC \\
\hline 10hb_nogap_A16 & GAAGCCTTAAATGGAAGCTTTACAAACAATTAACAACTAAA \\
\hline 10hb_nogap_A17 & CGCGAGGCGTAAAACAGAGAAAACAAAATTAATTACTTAATT \\
\hline 10hb_nogap_A18 & CAAGCAAATCTCAGATGTTTCAATTACCTGAAAATCAAAAT \\
\hline 10hb_nogap_B01 & CTTGTCAGACCACCCTCAGAGCCGGCCTTTACCAATGAAAC \\
\hline 10hb_nogap_B02 & ATAATTAAAGCCTCAGAGCCGCCAGGTCATATCACCAGTAG \\
\hline 10hb_nogap_B03 & TTCCAGCGATAACTTTGAAAGAGGTATTCATAGTCAGGACG \\
\hline 10hb_nogap_B04 & GCTAGTATCATAACGAGGCGCAGACGAATAAGGTGAATTACC \\
\hline 10hb_nogap_B05 & ATTATATTTTCTGTCTGGAAGTTTCCTTCAAAGACTATTATA \\
\hline 10hb_nogap_B06 & TAAATACTAATTGCTGTAGCTCAACGATTAGAAAAACGAGAA \\
\hline 10hb_nogap_B07 & CTGCCACACAACGCGCGGGGAGAGGGCCTGGCTAAAGAACGT \\
\hline 10hb_nogap_B08 & TCACGTGCCTATCGGGAAACCTGTCTGCCCCATAGGGTTGAG \\
\hline 10hb_nogap_B09 & CACGATACGTGCGTCTGAAATGGATAAATTAAATTTTAGACA \\
\hline 10hb_nogap_B10 & CAGAAATGCGCAGGAAAAACGCTCATCACTTGTTAGAATCAG \\
\hline 10hb_nogap_B11 & TTATACAAAGAATCATAATTACTAGAATTGAGAGCTAATGCA \\
\hline 10hb_nogap_B12 & CGATTTTCATCACCGTGTGATAAATGGCAGAGTAAAGTAATT \\
\hline
\end{tabular}




\begin{tabular}{|c|c|}
\hline 10hb_nogap_C01 & TTTCATAATCAAAATCTCTAACGGAAACTTGAGCCATTATCT \\
\hline 10hb_nogap_C02 & TAAGAGGTCATTTTTCAATCAGGGCGTTGAATCCCCCTTTGA \\
\hline 10hb_nogap_C03 & GGCCTTGCTGGTAATCTCTGAACAAGGCTTTGACGAGCTATC \\
\hline 10hb_nogap_C04 & ACGCATTAGATGCGAACGAGTAGAAAGGAAGCCCGAAAGAC \\
\hline 10hb_nogap_C05 & TTGTGGCCAAACGACCAGTAATAATCATCAGTGAGGCCACC \\
\hline 10hb_nogap_C06 & AGGGATAGCATAAGTTTCATTCAAAACGTCAGCGTCAGACT \\
\hline 10hb_nogap_C07 & AGTTTCGTCAAAAGGTGTTATTCAAGCAAAAGCCCCCTTAT \\
\hline 10hb_nogap_C08 & CAGACAGCCCCTTATTATACAGGTAAACGAAGACCTTCATC \\
\hline 10hb_nogap_C09 & GTCTTTCCAGATAATAAATTCAACTTATACCTACCCAAATC \\
\hline 10hb_nogap_C10 & TGCTAAACAAGATAGCCGCATAGTAATCATTGCTTGCCCTG \\
\hline 10hb_nogap_C11 & AAGATTGTATTTGAGTTTTTTGCCTTACCCTGCGAACCAGA \\
\hline 10hb_nogap_C12 & TTGTTAAAATAGGGTAACGTCCAAAGTTCAGGAGTACCTTT \\
\hline 10hb_nogap_C13 & TTTAACCAATCATTAGATACGTGAACCGTCTCCAGTGAGAC \\
\hline 10hb_nogap_C14 & CTTCCTGTAGTAGCAGCGCCGTAACCACTATCCTGAGAGAG \\
\hline 10hb_nogap_C15 & AACAACCCGTCCAAATATTAGAGCCCCGAGAGCAGGCGAAA \\
\hline 10hb_nogap_C16 & TGATGGCAATCTGAATCGTGTAGCTAAAGGGCCGTTGTAGC \\
\hline 10hb_nogap_C17 & CTTCTGAATAATCAAGATTAATGCTTCCTCGCCTGAGTAGA \\
\hline 10hb_nogap_C18 & ATTTGCACGTTTTAGCGATCCCATATAAGTCTACCAGTATA \\
\hline 10hb_nogap_C19 & AGGTTTAACGAGATATACGGCTGTCATGTTCAATCGCCATA \\
\hline 10hb_nogap_D01 & AACCAGATGGTCAGAACGAGTAGTATTCGACCTGCTCCATGT \\
\hline 10hb_nogap_D02 & CGAACCTTATATGGTGGTTCCGAAAAACGTTGCGCTCACTGC \\
\hline 10hb_nogap_D03 & AACTAAAGGAATTGCCTCAGCAGCGAAAATTTTTACAGGAAC \\
\hline 10hb_nogap_D04 & TGGGATAGGTCACGCTGCAAGGCGATAAATATCAACACGTAA \\
\hline 10hb_nogap_D05 & ATCACCGTACGCTGAGATAATAAGTTCACAAAACCGCCACC \\
\hline 10hb_nogap_D06 & GCCGTCGAGAGCTCAGTTAAGCGTGCAGTCTAGCCACCACC \\
\hline 10hb_nogap_D07 & TCAGCTTGCTCAACAACAATACGTAAAACACAACGGTGTAC \\
\hline 10hb_nogap_D08 & AAAAAAAAGGAGGCTTGACTAAAGAACAAAGCATAAGGGAA \\
\hline 10hb_nogap_D09 & TGAACGGTAAGCAATGCTTTTGCGGGTCAATATAACAGTTG \\
\hline 10hb_nogap_D10 & TCAGGTCATTGAAAGGCAGCTAAACTGAAAAAAATATGCAA \\
\hline 10hb_nogap_D11 & TGCCGGAGAGACCGTTCCAAAGAACATCCAAGAGCTTAATT \\
\hline 10hb_nogap_D12 & ATCGCACTCCCGCCATTGGATCCCATTGTTACGTATTGGGC \\
\hline 10hb_nogap_D13 & CTGCCAGTTTGGGCCTCACGGCCAGCATAAACTGCATTAAT \\
\hline 10hb_nogap_D14 & GTAACATTATAGTTGGCCAAATGATTCTGACATTGGCAGAT \\
\hline 10hb_nogap_D15 & ATTAAATCCTTAAAATATATTAACTTTGAATACCTACATTT \\
\hline 10hb_nogap_D16 & GATTTAGAAGGTCAATAACGAACCAAAACATATTACCGCCA \\
\hline 10hb_nogap_D17 & TGATGAAACATTTTTAATGAGAGATGATGCACTGTTTAGTA \\
\hline 10hb_nogap_D18 & CAGAGGCGAATAACCTTCGCTGAGTTTCAAATAAATAAGAA \\
\hline 10hb_nogap_E01 & GGTAATTACCATCATCGGCATTTTCCCCTCAGACAAATAAAT \\
\hline 10hb_nogap_E02 & GAGAGAAGAAATGACAAGAACCGGAACAGATGTCATCTTTGA \\
\hline 10hb_nogap_E03 & GCCAGCGATTTGCTGCTCATTCAGTGGTCAATTACAACGGAG \\
\hline 10hb_nogap_E04 & AGGCGAAGCAACGTTTTAATTCGAGATTCCATAACCTGTTTA \\
\hline 10hb_nogap_E05 & AATGCATAAATCTCCAACAGGTCAGATGTTTTGGTGGCATCA \\
\hline 10hb_nogap_E06 & GTTTTCCAACGGATTGCCCTTCACCCGGTTTGTCCGCTCACA \\
\hline 10hb_nogap_E07 & CTAAGTTCCAGGGTCCACGCTGGTTGTGCCAGGTGTAAAGCC \\
\hline 10hb_nogap_E08 & GCGCCGGTACGTCTGTCCATCACGCTATTTACCTGAAAGCGT \\
\hline 10hb_nogap_E09 & CCACGGAGCTAGATTAGTAATAACATGGAAATGGCTATTAGT \\
\hline 10hb_nogap_E10 & TAGAGCGCCTGCAACAGTAGGGCTTAAAAAGCAATCCAATCG \\
\hline 10hb_nogap_E11 & AGAACCAGACGCAACATGTAATTTAAAGGCGTTATATTTTAG \\
\hline 10hb_nogap_F01 & GTATTAAGAGTCAGGAGTAGGAACCCATGTAAAACGCAGAA \\
\hline 10hb_nogap_F02 & GCGGGGTTTTGGGTTGAAAACTACAACGCCTAAACGTATCAC \\
\hline 10hb_nogap_F03 & CCGACAATGATTCGAGGTAGCGTAACGATCTGAACTGGGTT \\
\hline 10hb_nogap_F04 & TCGGTCGCTGCTCCAAATAAATGAATTTTCTGAAGGAAAAC \\
\hline 10hb_nogap_F05 & GGATCGTCACCGAATAACAGTTTCAGCGGAGCCTTTTTCAT \\
\hline 10hb_nogap_F06 & TATTTTAAATTCGTAAATAATCAGAAAAGCCGAGCAAGGAG \\
\hline
\end{tabular}




\begin{tabular}{|c|c|}
\hline 10hb_nogap_F07 & AAAAGGGTGAGCCTGAGTATTTAAATTGTAACAGAGAGTAA \\
\hline 10hb_nogap_F08 & ATGATATTCAGGTAGCTAAATTTTTGTTAAAGAACACCCATA \\
\hline 10hb_nogap_F09 & AAGCGCCATTAGCCAGCCCATCAAAAATAATTAACATACAA \\
\hline 10hb_nogap_F10 & CGATCGGTGCGAGGGGATCATCAACATTAAAGTTTAACACC \\
\hline 10hb_nogap_F11 & GGGGGATGTGTTGGTGTTCCGTGGGAACAAAAGCCATACGG \\
\hline 10hb_nogap_F12 & ATATCTGGTCCATTTTGTATCATCATATTCCGAGCGTCCGG \\
\hline 10hb_nogap_F13 & GAAGGTTATCTTGCCCGTATAATCCTGATTGGCACCCATAA \\
\hline 10hb_nogap_F14 & GATTAGAGCCTATTAGAGGTTAGAACCTACCCGGGAGGCGTA \\
\hline 10hb_nogap_F15 & TGAATTACCTAACATCAAAATAAAGAAATTGTATTCTAATG \\
\hline 10hb_nogap_F16 & TGTGAGTGAATTATTCAAATATACAGTAACAGCGCCCACCA \\
\hline 10hb_4gap_004 & ACCGCAGTTCCAGACCAGGCGGGATACCGATAGTGGCA \\
\hline 10hb_4gap_005 & TTCAATGGCAAGGTAGCTGATATGCCGGAAACCAGAAT \\
\hline 10hb_4gap_006 & GGTTAAATACCGAGATAATACAATTTAACAATTTTCCG \\
\hline 10hb_4gap_011 & AAGATTGGGCTTGCTCGTTTAAAAGACAGCATCGTGTC \\
\hline 10hb_4gap_016 & TATAGGCAAAATCCGTGGCGTCTAAGTTGGGTAATCAC \\
\hline 10hb_4gap_021 & GCATTTTCGAGCCAAATACCGTTCTGAC \\
\hline 10hb_4gap_023 & AGTTTCCACCAGGGAGGTTGAGGCAGGAG \\
\hline 10hb_4gap_057 & GAGGATAGGCTGGCACCGGAACCAGCTGAATTTAAAAC \\
\hline 10hb_4gap_062 & AGCTTTTTCTTTTGCGGATGGCTTATAAATCATAATGG \\
\hline 10hb_4gap_067 & ATAAATACAAATTATCCAGAACAATCGCCATTAATGGG \\
\hline 10hb_4gap_070 & AGGGCGAATTTTGTCACAATCCCCTCATAACC \\
\hline 10hb_4gap_071 & CCTACCACATTATCGCAGTATGTTAGCGTAGCATAAGT \\
\hline 10hb_4gap_072 & AATCCAGGCCCACCGGGAGAATTAACTTCAGCTCTTAA \\
\hline 10hb_4gap_073 & AGGTAAAAATAATAACCTCCCGACTTGATATCAATGAG \\
\hline 10hb_4gap_074 & CCCTCATATTTCGGTATAAAATTGACAAACCACC \\
\hline 10hb_4gap_075 & TTACATCGGGAGAATTCATCGAGTACCGCAA \\
\hline 10hb_gap_A01 & CGGAAAGCCCAAGTTTAGTACCGCCAGAAACATGTGC \\
\hline 10hb_gap_A02 & TACATCCAGTACTATAAGTATAGCCCGATTAGGGATG \\
\hline 10hb_gap_A03 & GACTCTCATAGTTGAATTTCTTAAACAGCTTATTCCA \\
\hline 10hb_gap_A04 & ACGCAACGTTAGAGGAGCCTTTAATTAACCGATAAGT \\
\hline 10hb_gap_A05 & AAGCACTTTCAATAATTTTTTCACGTCCGCTTTACG \\
\hline 10hb_gap_A06 & GAAATCCGTAGTTTGACCAAGGATACCAGACTATCT \\
\hline 10hb_gap_A07 & AATAGCGGTTGAACTAGCATGTCAATGAACCCTTTCA \\
\hline 10hb_gap_A08 & AAGAAAAGCAAAAGTCTGGAGCAAACGGTAAAGAAC \\
\hline 10hb_gap_A09 & GTCAGTCGCATTATTTTTGAGAGATCTCACCATCAA \\
\hline 10hb_gap_A10 & AAGCGAGGAACGTTTCCGGCACCGCTTCTGGAAATTT \\
\hline 10hb_gap_A11 & GAAAACCAGCTTCGACGACAGTATCGGTTGGGATGC \\
\hline 10hb_gap_A12 & CCAATCGGATTCAGATGGGCGCATCGAGCTGGCCAG \\
\hline 10hb_gap_A13 & ATTAATTGGGGACATTCCTGAACCAGAAAGGGTTAC \\
\hline 10hb_gap_A14 & CCTAAGCGGAATCGGAACAAAGAAACACCCTCACACC \\
\hline 10hb_gap_A15 & TTATCTCATCAAAACGTTATTAATTTAAGGAATTGC \\
\hline 10hb_gap_A16 & CTTAAATGGAAGCTTTACAAACAATTAACAACTAAA \\
\hline 10hb_gap_A17 & GGCGTAAAACAGAGAAAACAAAATTAATTACTTAATT \\
\hline 10hb_gap_A18 & AAATCTCAGATGTTTCAATTACCTGAAAATCAAAAT \\
\hline 10hb_gap2_A01 & CGGAAAGCCCAAGTTTAGTACCGCCAGAAACATGTG \\
\hline 10hb_gap2_A02 & TACATCCAGTACTATAAGTATAGCCCGATTAGGGAT \\
\hline 10hb_gap2_A04 & ACGCAACGTTAGAGGAGCCTTTAATTAACCGATAAG \\
\hline 10hb_gap2_A06 & TCCGTAGTTTGACCAAGGATACCAGACTATCT \\
\hline 10hb_gap2_A07 & AATAGCGGTTGAACTAGCATGTCAATGAACCCT \\
\hline 10hb_gap2_A13 & ATTGGGGACATTCCTGAACCAGAAAGGGTTAC \\
\hline 10hb_gap2_A14 & CCTAAGCGGAATCGGAACAAAGAAACACCCTCA \\
\hline 10hb_gap_B01 & CTTGTCAGACCACCCTCAGAGCCGGCCTTTACCAAT \\
\hline 10hb_gap_B02 & ATAATTAAAGCCTCAGAGCCGCCAGGTCATATCACC \\
\hline 10hb_gap_B03 & TTCCAGCGATAACTTTGAAAGAGGTATTCATAGTCA \\
\hline 10hb_gap_B04 & GCTAGTATCATAACGAGGCGCAGACGAATAAGGTGAA \\
\hline 10hb_gap_B05 & ATTATATTTTCTGTCTGGAAGTTTCCTTCAAAGACTA \\
\hline 10hb_gap_B06 & TAAATACTAATTGCTGTAGCTCAACGATTAGAAAAAC \\
\hline
\end{tabular}




\begin{tabular}{|c|c|}
\hline 10hb_gap_B07 & CTGCCACACAACGCGCGGGGAGAGGGCCTGGCTAAAG \\
\hline 10hb_gap_B08 & TCACGTGCCTATCGGGAAACCTGTCTGCCCCATAGGG \\
\hline 10hb_gap_B09 & CACGATACGTGCGTCTGAAATGGATAAATTAAATTTT \\
\hline 10hb_gap_B10 & CAGAAATGCGCAGGAAAAACGCTCATCACTTGTTAGA \\
\hline 10hb_gap_B11 & TTATACAAAGAATCATAATTACTAGAATTGAGAGCTA \\
\hline 10hb_gap_B12 & CGATTTTCATCACCGTGTGATAAATGGCAGAGTAAAG \\
\hline 10hb_gap2_B01 & GTCAGACCACCCTCAGAGCCGGCCTTTACCAAT \\
\hline 10hb_gap2_B02 & ATTAAAGCCTCAGAGCCGCCAGGTCATATCACC \\
\hline 10hb_gap2_B03 & CAGCGATAACTTTGAAAGAGGTATTCATAGTCA \\
\hline 10hb_gap2_B04 & GTATCATAACGAGGCGCAGACGAATAAGGTGAA \\
\hline 10hb_gap2_B05 & TATTTTCTGTCTGGAAGTTTCCTTCAAAGACTA \\
\hline 10hb_gap2_B06 & TACTAATTGCTGTAGCTCAACGATTAGAAAAAC \\
\hline 10hb_gap2_B07 & CACACAACGCGCGGGGAGAGGGCCTGGCTAAAG \\
\hline 10hb_gap2_B08 & GTGCCTATCGGGAAACCTGTCTGCCCCATAGGG \\
\hline 10hb_gap2_B09 & ATACGTGCGTCTGAAATGGATAAATTAAATTTT \\
\hline 10hb_gap2_B10 & AATGCGCAGGAAAAACGCTCATCACTTGTTAGA \\
\hline 10hb_gap2_B11 & ACAAAGAATCATAATTACTAGAATTGAGAGCTA \\
\hline 10hb_gap2_B12 & TTTCATCACCGTGTGATAAATGGCAGAGTAAAG \\
\hline 10hb_gap_C01 & TAATCAAAATCTCTAACGGAAACTTGAGCCATTATCT \\
\hline 10hb_gap_C02 & GGTCATTTTTCAATCAGGGCGTTGAATCCCCCTTTGA \\
\hline 10hb_gap_C03 & TGCTGGTAATCTCTGAACAAGGCTTTGACGAGCTATC \\
\hline 10hb_gap_C04 & ACGCATTAGATGCGAACGAGTAGAAAGGAAGCCCGA \\
\hline 10hb_gap_C05 & TTGTGGCCAAACGACCAGTAATAATCATCAGTGAGG \\
\hline 10hb_gap_C06 & TAGCATAAGTTTCATTCAAAACGTCAGCGTC \\
\hline 10hb_gap_C07 & CGTCAAAAGGTGTTATTCAAGCAAAAGCCCC \\
\hline 10hb_gap_C08 & AGCCCCTTATTATACAGGTAAACGAAGACCT \\
\hline 10hb_gap_C09 & TCCAGATAATAAATTCAACTTATACCTACCC \\
\hline 10hb_gap_C10 & AACAAGATAGCCGCATAGTAATCATTGCTTG \\
\hline 10hb_gap_C11 & TGTATTTGAGTTTTTTGCCTTACCCTGCGAA \\
\hline 10hb_gap_C12 & AAAATAGGGTAACGTCCAAAGTTCAGGAGTA \\
\hline 10hb_gap_C13 & CCAATCATTAGATACGTGAACCGTCTCCAGT \\
\hline 10hb_gap_C14 & TGTAGTAGCAGCGCCGTAACCACTATCCTGA \\
\hline 10hb_gap_C15 & CCCGTCCAAATATTAGAGCCCCGAGAGCAGG \\
\hline 10hb_gap_C16 & GCAATCTGAATCGTGTAGCTAAAGGGCCGTT \\
\hline 10hb_gap_C17 & GAATAATCAAGATTAATGCTTCCTCGCCTGA \\
\hline 10hb_gap_C18 & CACGTTTTAGCGATCCCATATAAGTCTACCA \\
\hline 10hb_gap_C19 & TAACGAGATATACGGCTGTCATGTTCAATCG \\
\hline 10hb_gap_D01 & AACCAGATGGTCAGAACGAGTAGTATTCGACCTGCTC \\
\hline 10hb_gap_D02 & CGAACCTTATATGGTGGTTCCGAAAAACGTTGCGCTC \\
\hline 10hb_gap_D03 & AAGGAATTGCCTCAGCAGCGAAAATTTTTACAGGAAC \\
\hline 10hb_gap_D04 & TAGGTCACGCTGCAAGGCGATAAATATCAACACGTAA \\
\hline 10hb_gap_D05 & CGTACGCTGAGATAATAAGTTCACAAAACCG \\
\hline 10hb_gap_D06 & CGAGAGCTCAGTTAAGCGTGCAGTCTAGCCA \\
\hline 10hb_gap_D07 & TTGCTCAACAACAATACGTAAAACACAACGG \\
\hline 10hb_gap_D08 & AAAGGAGGCTTGACTAAAGAACAAAGCATAA \\
\hline 10hb_gap_D09 & GGTAAGCAATGCTTTTGCGGGTCAATATAAC \\
\hline 10hb_gap_D10 & TCATTGAAAGGCAGCTAAACTGAAAAAAATA \\
\hline 10hb_gap_D11 & GAGAGACCGTTCCAAAGAACATCCAAGAGCT \\
\hline 10hb_gap_D12 & ACTCCCGCCATTGGATCCCATTGTTACGTAT \\
\hline 10hb_gap_D13 & AGTTTGGGCCTCACGGCCAGCATAAACTGCA \\
\hline 10hb_gap_D14 & ATTATAGTTGGCCAAATGATTCTGACATTGG \\
\hline 10hb_gap_D15 & ATCCTTAAAATATATTAACTTTGAATACCTA \\
\hline 10hb_gap_D16 & AGAAGGTCAATAACGAACCAAAACATATTAC \\
\hline 10hb_gap_D17 & AAACATTTTTAATGAGAGATGATGCACTGTT \\
\hline 10hb_gap_D18 & GCGAATAACCTTCGCTGAGTTTCAAATAAAT \\
\hline 10hb_gap2_D01 & AGATGGTCAGAACGAGTAGTATTCGACCTGCTC \\
\hline 10hb_gap2_D02 & CCTTATATGGTGGTTCCGAAAAACGTTGCGCTC \\
\hline
\end{tabular}




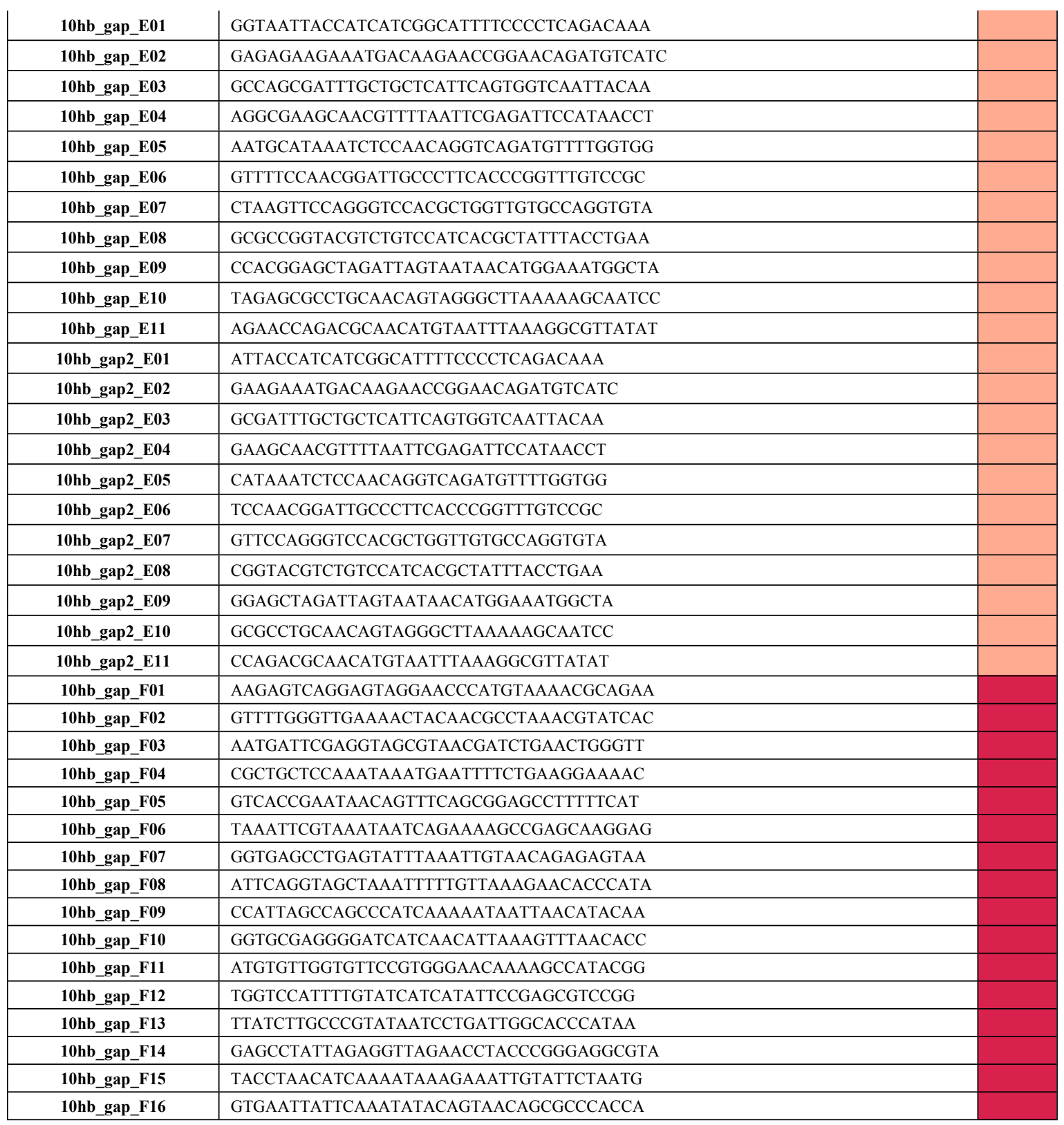

\section{Supplementary Table 7. Staple list of $10 \mathrm{HB}$ designs.}




\section{Supplementary references}

1. Douglas, S. M.; Marblestone, A. H.; Teerapittayanon, S.; Vazquez, A.; Church, G. M.; Shih, W. M. Rapid Prototyping of 3D DNA-Origami Shapes with Cadnano. Nucleic Acids Res. 2009, 37, 5001-5006.

2. Douglas, S. M.; Dietz, H.; Liedl, T.; Hogberg, B.; Graf, F.; Shih, W. M. Self-Assembly of DNA into Nanoscale Three-Dimensional Shapes. Nature 2009, 459, 414-418.

3. Ke, Y.; Douglas, S. M.; Liu, M.; Sharma, J.; Cheng, A.; Leung, A.; Liu, Y.; Shih, W. M.; Yan, H. Multilayer DNA Origami Packed on a Square Lattice. J. Am. Chem. Soc. 2009, 131, 15903-15908.

4. Kim, D.-N.; Kilchherr, F.; Dietz, H.; Bathe, M. Quantitative Prediction of 3D Solution Shape and Flexibility of Nucleic Acid Nanostructures. Nucleic Acids Res. 2012, 40, 2862-2868.

5. Kim, Y.-J.; Lee, C.; Lee, J. G.; Kim, D.-N. Configurational Design of Mechanical Perturbation for Fine Control of Twisted DNA Origami Structures. ACS Nano 2019, 13, 6348-6355.

6. Sedeh, R. S.; Bathe, M.; Bathe, K.-J. The Subspace Iteration Method in Protein Normal Mode Analysis. J. Comput. Chem. 2010, 31, 66-74.

7. Bathe, K.-J.; Ramaswamy, S. An Accelerated Subspace Iteration Method. Comput. Meth. Appl. Mech. Eng. 1980, 23, 313-331.

8. Thomson, W. T. Euler Equation for Beams. In Theory of Vibration with Applications. CrC Press: New York, 2018; pp 334-339.

9. Jorgensen, W. L.; Chandrasekhar, J.; Madura, J. D.; Impey, R. W.; Klein, M. L. Comparison of Simple Potential Functions for Simulating Liquid Water. J. Chem. Phys. 1983, 79, 926-935.

10. Lee, J. Y.; Kim, Y.-J.; Lee, C.; Lee, J. G.; Yagyu, H.; Tabata, O.; Kim, D.-N. Investigating the Sequence-Dependent Mechanical Properties of DNA Nicks for Applications in Twisted DNA Nanostructure Design. Nucleic Acids Res. 2019, 47, 93-102.

11. Phillips, J. C.; Braun, R.; Wang, W.; Gumbart, J.; Tajkhorshid, E.; Villa, E.; Chipot, C.; Skeel, R. D.; Kalé, L.; Schulten, K. Scalable Molecular Dynamics with NAMD. J. Comput. Chem. 2005, 26, 1781-1802.

12. Hart, K.; Foloppe, N.; Baker, C. M.; Denning, E. J.; Nilsson, L.; MacKerell, A. D. Optimization of the CHARMM Additive Force Field for DNA: Improved Treatment of the BI/BI Conformational Equilibrium. J. Chem. Theory Comput. 2012, 8, 348-362.

13. Essmann, U.; Perera, L.; Berkowitz, M. L.; Darden, T.; Lee, H.; Pedersen, L. G. A Smooth Particle Mesh Ewald Method. J. Chem. Phys. 1995, 103, 8577-8593.

14. Lu, X. J.; Olson, W. K. 3DNA: A Software Package for the Analysis, Rebuilding and Visualization of Three-Dimensional Nucleic Acid Structures. Nucleic Acids Res. 2003, 31, 5108-5121.

15. Brooks, B. R.; Janežič, D.; Karplus, M. Harmonic Analysis of Large Systems. I. Methodology. J. Comput. Chem. 1995, 16, 1522-1542.

16. Lee, C.; Lee, J. Y.; Kim, D.-N. Polymorphic Design of DNA Origami Structures through Mechanical Control of Modular Components. Nat. Commun. 2017, 8, 2067.

17. Humphrey, W.; Dalke, A.; Schulten, K. VMD: Visual Molecular Dynamics. J. Mol. Graph. 1996, 14, 33-38.

18. Yoo, J.; Aksimentiev, A. In Situ Structure and Dynamics of DNA Origami Determined through 
Molecular Dynamics Simulations. Proc. Natl. Acad. Sci. U.S.A. 2013, 110, 20099-20104. 